PNL-6734

UC-11,41

2

\title{
A Review of Historical Data on the Radionuclide Content of Soil Samples Collected from the Hanford Site and Vicinity
}

K. R. Price

November 1988

-

Prepared for the U.S. Department of Energy under Contract DE-AC06-76RLO 1830

Pacific Northwest Laboratory Operated for the U.S. Department of Energy by Battelle Memorial Institute 


\title{
DISCLAIMER
}

This report was prepared as an account of work sponsored by an agency of the United States Government. Neither the United States Government nor any agency thereof, nor Battelle Memorial Institute, nor any or their employees, makes any warranty, expressed or implied, or assumes any legal liability or responsibility for the accuracy, completeness, or usefulness of any information, apparatus, product, or process disclosed, or represents that its use would not infringe privately owned rights. Reference herein to any specific commercial product, process, or service by trade name, trademark, manufacturer, or otherwise does not necessarily constitute or imply its endorsement, recommendation, or favoring by the United States Government or any agency thereof, or Battelle Memorial Institute. The views and opinions of authors expressed herein do not necessarily state or reflect those of the United States Covernment or any agency thereof.

\author{
PACIFIC NORTHWEST LABORATORY \\ operated by \\ BATTELLE MEMORIAL INSTITUTE \\ for the \\ UNITED STATES DEPARTMENT OF ENERCY \\ under Contract DE-AC06-76RLO /830
}

Printed in the United States of America
Available from
National Technical Information Service
United States Department of Commerce
5285 Port Royal Road
5pringfield, Virginia 22161
NTIS Price Codes
Microfiche A01
Printed Copy
Pdges
$001-025$
$026-050$
051.075


PNL-6734

UC $-11,41$

A REVIEW OF HISTORICAL DATA ON THE RADIONUCLIDE CONTENT OF SOIL SAMPLES COLLECTED FROM THE HANFORD SITE AND VICINITY

K. R. Price

November 1988

Prepared for the U.S. Department of Energy under Contract DE-ACO6-76RLO 1830

Pacific Northwest Laboratory Richland, Washington 99352 
,

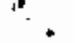




\section{PREFACE}

In February 1986, the U.S. Department of Energy (DOE) made available to the public numerous documents containing historical information about effluent releases and environmental monitoring at the Hanford Site. The Hanford Health Effects Review Panel reviewed this information and made 33 recommendations. (a) Environmental Monitoring Recommendation C.6 from the final report prepared by the Review Panel stated: "The panel encourages expanding this program [soil sampling] as a means of estimating the amount of radionuclides deposited at the Hanford Site since the beginning of operations." The recommendation further stated: "Radionuclides to be evaluated should include (but not be limited to) isotopes of plutonium, americium, iodine, strontium, and cesium." The DOE response to the recommendation was that existing data should be reviewed and summarized before undertaking new sampling activities. The information presented in this document is in response to Recommendation C.6, and reviews and sumnarizes soil sampling results obtained over the years.

\footnotetext{
(a) "Report of The Hanford Health Effects Review Panel and Recommendations of the Sponsoring Agencies," October 1987, Washington State Department of Social and Health Services, Olympia, Washington.
} 
. 


\section{SUMMARY}

The measurement of radioactive materials in soil samples collected from the environs of the Hanford Site has been a routine part of environmental monitoring since 1971. Soil samples have also been collected and analyzed for special-purpose studies. The main objective of this report is to review and sumbarize the historical record of soil sampling results related to environmental monitoring from the late 1950s through 1987. Other objectives are to publish previously unpublished data and to consolidate results from routine environmental monitoring and special studies into a single document.

The sources of information reviewed included the environmental monitoring computerized data base, historical files, annual environmental monitoring reports, and other Hanford documents. A rigorous evaluation of data from these sources was not conducted, but several observations were made during the course of review:

- Soil sampling has not revealed any gross contamination of the offsite environs from past Hanford operations.

- Some onsite soil sampling locations near facilities have received contamination from past operations at Hanford.

- Results have not indicated a gradual buildup or depletion of contamination at any given soil sampling location.

- Results were noted to vary among aliquots analyzed from a single sample or among multiple samples collected at a single location.

- Hanford-derived radionuclides have been difficult to identify in the presence of worldwide fallout. 



\section{ACKNOWLEDGMENTS}

The author greatfully acknowledges the assistance of L. E. Bisping and R. W. Hanf, Jr. for preparation of the data tables for this report. G. L. Dirkes prepared the final manuscript, and M. E. Strong provided careful editing assistance. 



\section{CONTENTS}

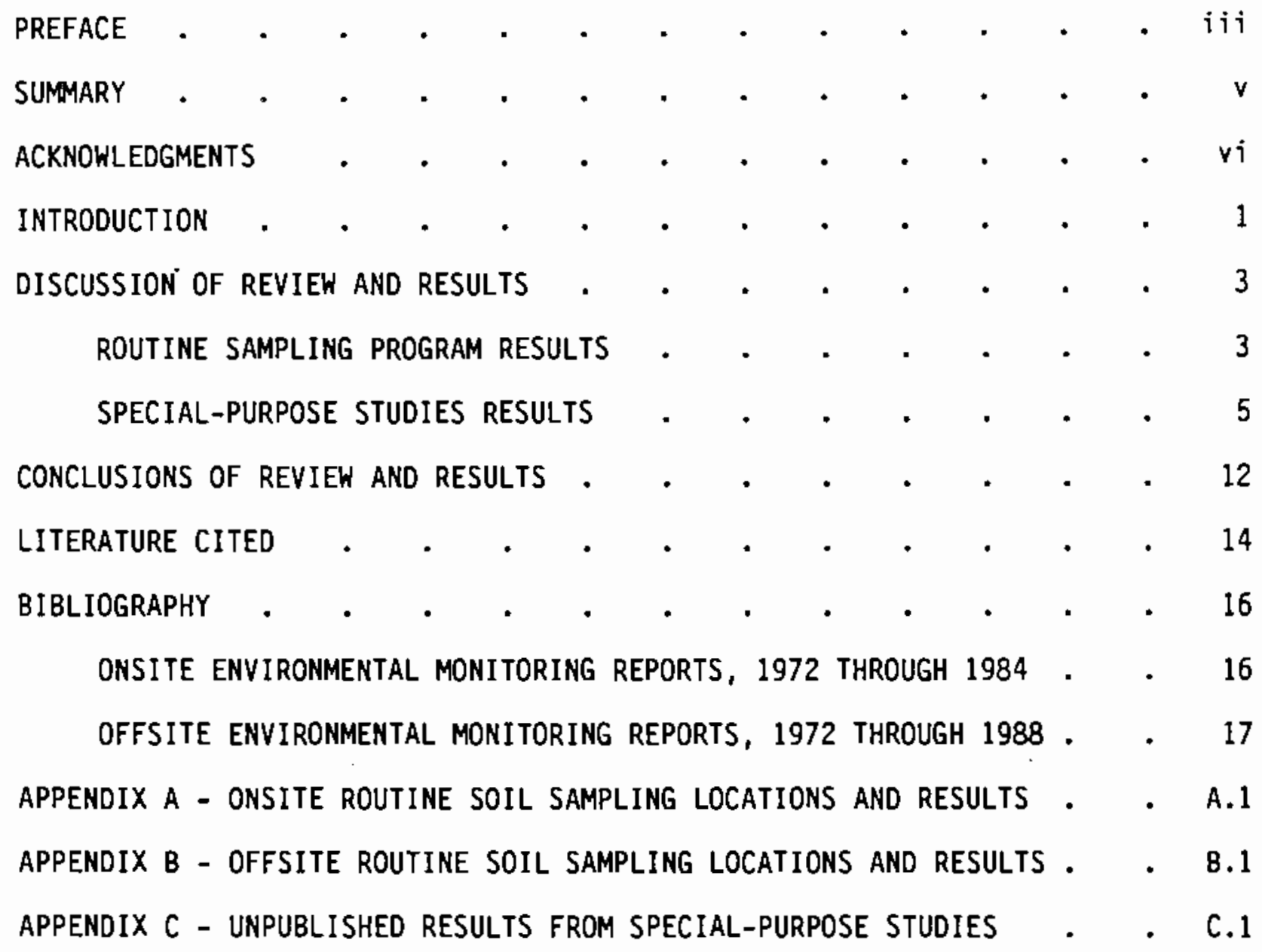


FIGURES

A.1. Onsite Routine Soil Sampling Locations, 1971

through 1987 . . . . . . . . . . A.4

B.1. Offsite Routine Soil Sampling Locations, 1971

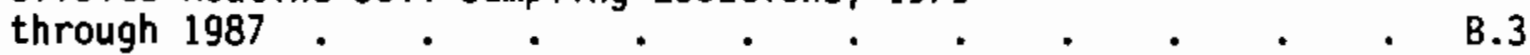

C.1. Unpublished Results from a Special-Purpose Study

Downwind of the Hanford Site, May 1987. . . . . . c.1

C.2. Unpublished Results from a Special-Purpose Study Along
the Southern Perimeter of the Hanford Site, June 1985. . . C.2

C.3. Unpublished Results from a Special-Purpose Study East

of the 200 West Area, August 1985 . . . . . . . . C.3

C.4. Unpublished Results from a Special-Purpose Study North C.5. Unpublished Results from a Special-Purpose Study South
of the B-C Cribs Controlled Area . . . . . . . C.5

C.6. Unpublished Results from a Special-Purpose Study at the Proposed Puget Sound Power and Light Company Site, August 1985

C.7. Unpublished Results from a Special-Purpose Study at Various Onsite and Offsite Locations, February and March 1970 .

\section{TABLES}

A.1. Onsite Routine Soil Sampling Locations, 1971 through 1987

A.2. Strontium-90 Concentrations in Soil at Onsite Locations . . A.5

A.3. Cesium-137 Concentrations in Soil at Onsite Locations . . . A.14

A.4. Plutonium-238 Concentrations in Soil at Onsite Locations . . A.23

A.5. Plutonium-239,240 Concentrations in Soil at Onsite Locations

A.6. Americium-241 Concentrations in Soil at Onsite Locations . . A.41

A.7. Uranium Concentrations in Soil at Onsite Locations $\quad{ }^{*} \quad$ - . A.44 


\section{TABLES (cont)}

B.1. Offsite Routine Soil Sampling Locations, 1971

Through 1987 . $\quad$ •

B.2. Strontium-90 Concentrations in Soil at offsite

Locations . . . . • . • . . . . B.4

B.3. Cesium-137 Concentrations in Soil at 0ffsite
Locations . . . . . . . . B.9

B.4. Plutonium-238 Concentrations in Soil at Offsite
Locations . . . . . B.14

B.5. Plutonium-239,240 Concentrations in Soil at offsite

B.6. Americium-241 Concentrations in Soil at Offsite Locations . . . . . . . . . . . B.24

B.7. Uranium Concentrations in Soil at Offsite Locations . . . B.26 


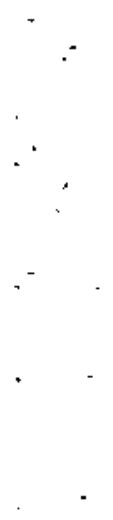




\section{INTRODUCTION}

The U.S. Department of Energy's (DOE) Hanford Site is located in a rural region of southeastern Washington and occupies an area of about $560 \mathrm{mi}^{2}$. The Columbia River flows through the northern edge of the Site and forms part of the eastern boundary. The southern boundary of the Site includes the Rattlesnake Hills, which exceed $3200 \mathrm{ft}$ in elevation. The Hanford Project was established in 1943 and was originally designed, built, and operated to produce plutonium for nuclear weapons.

All DOE sites are required to monitor for the presence of radioactive materials in the environment and to report results on an annual basis. The Pacific Northwest Laboratory (PNL) (a) is responsible for sitewide environmental monitoring at Hanford. A routine part of sitewide environmental monitoring since 1971 has been the measurement of radioactive materials in soil samples collected from the onsite as well as offsite environs. In addition to routine sampling, several special-purpose studies for environmental monitoring have been conducted over the years by Hanford scientists.

The main objective of this report is to review and summarize the historical record of results from routine environmental monitoring and special-purpose studies related to environmental monitoring from the late 1950s through 1987. Other objectives are to publish previously unpublished data and to consolidate results from routine environmental monitoring and special studies into a single document. The sources of information reviewed consisted of the environmental monitoring computerized data base, historical files, annual environmental monitoring reports, and other Hanford documents. Except where noted, the conclusions expressed in this report were taken from the reports and documents reviewed and represent the interpretations of the original author(s).

The main body of this report consists of a review of published and unpublished data for radiochemical analyses of soil samples collected on and

(a) PNL is operated for the U.S. Department of Energy by the Battelle Memorial Institute. 
off the Site. Appendix A lists onsite sampling locations and includes a map showing where onsite samples have been routinely collected over the years. Included also is a complete listing of analytical results for ${ }^{90} \mathrm{Sr},{ }^{137} \mathrm{Cs}$, $238 \mathrm{Pu}_{\mathrm{u}}, 239,240 \mathrm{Pu},{ }^{241} \mathrm{Am}$, and uranium. Appendix B contains similar information for offsite sampling locations. Previously unpublished results from special-purpose studies are given in Appendix $C$. 


\section{DISCUSSION AND REVIEW OF RESULTS}

Results from soil samples from onsite and offsite locations collected and analyzed over the past 30 years are summarized into two categories for review: results from the routine sampling program and results from specialpurpose studies.

\section{ROUTINE SAMPLING PROGRAM RESULTS}

The measurement of radioactive materials in soil samples collected from onsite (excluding operating areas) and offsite locations has been a routine part of sitewide environmental monitoring at Hanford since 1971. Monitoring has been documented in annual reports indicating results and conclusions. All published references listed in this report are compiled under LITERATURE CITED; other published annual reports that include soil sampling results from routine environmental monitoring are listed under BIBLIOGRAPHY.

The various reasons for routinely collecting and analyzing soil samples have been described over the years:

- Discover obvious geographical patterns of contamination

- Compare results from onsite locations with those from around the Hanford Site perimeter and with distant locations

- Identify trends or the gradual buildup of contamination in surface soils

- Monitor areas of known contamination for indications of a change of status

- Test the data for unusual results that may reveal previously undetected releases of radioactive materials during Site operations.

Radiochemical analyses dating from 1971 through 1987 have included ${ }^{90} \mathrm{Sr}$, ${ }^{137} \mathrm{Cs},{ }^{238} \mathrm{Pu}$, and $239,240 \mathrm{Pu}$. Americium-241 analyses began in 1982 (three locations were sampled and analyzed once in 1977). Uranium analyses began in 1973. Table A.1, Appendix A, lists the 12 onsite sampling locations and Table B.1, Appendix 8, lists the 24 offsite sampling locations. Samples were collected at 0-to 1-in. and 1-to 2-in. depths at each location in 1971 and 1972. Samples were also collected at 2- to 4-, 4- to 8-, and 8- to 12-in. depths at three locations in 1973. All other samples listed in the tables 
represent the upper 1-in. layer of surface soil. Figures A.1, Appendix A, and B.1. Appendix B, show the onsite and offsite locations, respectively, where samples have been collected over the years.

Tables A.2 through A.7, Appendix A, list all onsite sampling results, and Tables B.2 through B.7 list all offsite sampling results stored in the environmental monitoring computerized data base for the period 1971 through 1987. The data consist of the sampling date and the result \pm 2 standard deviations (counting uncertainty) for ${ }^{90} \mathrm{Sr},{ }^{137} \mathrm{Cs},{ }^{238} \mathrm{Pu}_{1}, 23 \overline{9}, 240 \mathrm{Pu},{ }^{241} \mathrm{Am}$, and uranium analyses.

Analytical results for the two samples collected at each location in 1971 and 1972 were published in the onsite annual environmental monitoring reports for the respective years (Bramson and Corley 1972a; Bramson, Corley, and Nees 1973). The authors noted a greater radionuclide concentration in the first l-in. layer of soil compared to the second 1-in. layer at most locations. Some exceptions were acknowledged where an apparent greater concentration was measured in the second $1-i n$. layer. No reason was given for the exceptions. 1n 1973, along with 1-in. layer samples taken from onsite and offsite locations, additional samples were taken from 200 East Southeast (ESE), 200 West Area CP 60 (a), and 200 East Area CP 59. These additional samples were collected from the 2- to 4-, 4- to 8-, and 8- to $12-$ in. layers. Results were published in the onsite annual report for 1973 (Nees and Corley 1975). The concentrations of radionuclides were again noted to be greater in the first 1-in. layer of soil than at any lower depth at most locations. No conclusions were stated by the authors for results from these profile samples, but the data show an unexpected presence of radionuclides in some of the deeper samples. The method used to collect the samples was not described.

A PNL report was prepared in 1977 that compared soil sample results for 1971 through 1976 (Miller, Fix, and Bramson 1977). The report concluded that, with the exception of a few locations, the data indicated that Hanford operations had made no significant contribution to existing radionuclide concentrations in soil samples. Most of the radioactivity measured in

(a) Some areas monitored routinely were designated as a Control Plot or "CP." 
samples was attributed to natural causes or worldwide atmospheric fallout. Three samples from onsite locations showed traces of contamination. One sample from an island near the Site boundary in the Columbia River north of Richland, Washington, showed elevated levels of ${ }^{60}$ Co resulting from cooling water discharges from the Hanford reactors. Results from the limited number of profile samples collected in 1971, 1972, and 1973 were not discussed.

Another PNL report sumarized routine soil sample results for the 5 years before restart of the PUREX (plutonium and uranium extraction) plant in 1983 (Sula and Price 1983). A large variability in the data was noted, and it was difficult to determine if there was a time-dependent trend in the data for the 5-year period reviewed. The authors concluded that surface soil at some of the 200 Area's sampling locations appeared to contain elevated levels of radioactive materials when compared to offsite locations. No other reviews of results from the routine soil sampling program have been published since sampling began in 1971.

\section{SPECIAL-PURPOSE STUDIES RESULTS}

Several special-purpose studies were completed between 1956 and 1987. The following is a chronological summary of these studies. Published results are listed under LITERATURE CITED. Unpublished results obtained from letter reports stored in historical files are reproduced in Appendix $C$ as noted. 1987 - Samples of surface soil (upper inch) were collected in 1987 at the same offsite locations downwind of Hanford that were sampled in 1986 (see entry for 1986). Samples were also collected from the communities of Moses Lake, Conne11, Othello, Washtucna, Walla Walla, and Yakima, Washington, and McNary, Oregon. The 1986 and 1987 samples were analyzed for the ${ }^{240} \mathrm{Pu}$ and ${ }^{239} \mathrm{pu}$ isotopes using the same special and sensitive mass spectrographic method used before to detect the presence of Hanford-derived plutonium (see entry for 1984). The average isotopic ratio of ${ }^{240} \mathrm{Pu}$ to ${ }^{239} \mathrm{Pu}$ for the special samples collected in 1986 and 1987 was $0.176 \pm 0.003$. The average ratio indicative of worldwide fallout in soil has been reported as $0.176 \pm$ 0.014 . The study concluded that the $239,240 \mathrm{Pu}$ detected in the samples was primarily from worldwide fallout. Results were reported in the 
1987 annual environmental monitoring report (Jaquish and Mitchell 1988). The 1987 samples were also analyzed for ${ }^{90} \mathrm{Sr},{ }^{137} \mathrm{Cs},{ }^{238} \mathrm{Pu}$, $239,240 \mathrm{Pu}$, and uranium. Results are sunmarized in Figure C.1 of Appendix $C$.

1986 - Samples of surface soil (upper inch) from eight locations downwind of the Hanford Site were collected from rural areas north of Pasco, Washington. Samples were collected and analyzed in the same manner as routine samples. No unusual conditions were detected. Soil concentrations of ${ }^{90} \mathrm{Sr},{ }^{137} \mathrm{Cs}, 239,240 \mathrm{Pu}$, and uranium were similar to nearby routine sampling locations and typical of worldwide fallout. Results were reported in the 1986 annual environmental monitoring report (PNL 1987).

1985 - Ten samples of surface soil (upper inch) were collected from the southern perimeter of the Hanford Site at 0.5-mi intervals along Highway 240 and from Horn Rapids Road from the junction of Highway 224 (Benton City highway) to Stevens Drive, Richland, Washington. Samples were collected and analyzed in the same manner as routine samples. Soil concentrations of ${ }^{90} \mathrm{Sr},{ }^{137} \mathrm{Cs}$, and $239,240 \mathrm{Pu}$ reflected worldwide fallout and were typical for the area when compared to nearby and distant routine sampling locations as published in the 1985 annual report (Price 1986). Results are summarized in Figure $C .2$ of Appendix $C$.

- Eight samples of surface soil (upper inch) were collected on Site east of the 200 West Area. Samples were collected and analyzed in the same manner as routine samples. Results for concentrations of $239,240 \mathrm{Pu}$ indicated that the spread of contamination known to exist in the area (routine sampling location "East of 200 West Gate") occurred in an easterly direction from the 200 West Area. Results are summarized in Figure C.3 of Appendix C.

- Four samples of surface soil (upper inch) were collected on Site north of the 200 East Area. Samples were collected and analyzed in the same manner as routine samples. Soil concentrations of ${ }^{90} \mathrm{Sr}$ and ${ }^{137} \mathrm{Cs}$ were known to be elevated in this area (routine sampling location "200 East Northcentral" (ENC). Results indicated that the spread of 
contamination extended in a northerly direction from the 200 East Area. Results are summarized in Figure C.4 of Appendix C.

- Two samples of surface soil (upper inch) were collected south of the B-C Cribs Controlled Area. The B-C Cribs Controlled Area is south of the 200 East Area. Samples were collected and analyzed in the same manner as routine samples. No spread of contamination from the B-C Cribs Controlled Area was detected. Soil concentrations of ${ }^{90} \mathrm{Sr}$, ${ }^{137} \mathrm{Cs}$, and $239,240 \mathrm{Pu}$ were typical for the onsite locations outside of operating areas. Results are summarized in Figure C.5 of Appendix C.

1984 - All samples of surface soil (upper inch) routinely collected for analysis in 1984, 1983, and 1982, plus five routine samples collected in 1978, were analyzed for the individual isotopes of plutonium using a special method. Routine analyses measured the amount of plutonium in the sample as 239,240 Pu but could not distinguish Hanford-derived plutonium from plutonium contained in worldwide fallout. Results from mass spectrographic analyses for the ${ }^{239} \mathrm{Pu}$ and ${ }^{240} \mathrm{Pu}$ isotopes were used to estimate the percentage of Hanford-derived plutonium in each soil sample. Results from these special analyses indicated that traces of Hanford-derived plutonium historically have been added to the total amount of plutonium detected in samples collected from onsite, onsite-perimeter, and some offsite locations.

The onsite location of maximum amount of Hanford-derived plutonium was near the 200 West Area (routine sampling location "East of 200 West Gate"). The offsite location of maximum Hanford-derived plutonium was near Benton City (routine sampling location "Benton City"). Soil samples from the Sunnyside area contained virtually no Hanford-derived plutonium. Results were listed in a data table and variability was apparent. Results for samples collected from the same location on successive years differed by as much as a factor of 3 . A factor of 1.6 was noted as the maximum difference between duplicate samples collected at the same time from the same location. Results from this special study were reported in the 1985 annual environmental monitoring report (Price 1986). 
1982 - A special study was conducted on land located off Site across the Columbia River from the 300 Area. Samples of surface soil (upper inch) from the study area were analyzed for uranium and nonradioactive heavy metals (lead, silver, zinc, and copper). Results for the study area were compared to results for samples collected at control sites located both on and off the Hanford Site. The study concluded that operations at Hanford had not resulted in a detectable impact off Site on land located across the River from the 300 Area. Results were reported in a PNL document (Price and Kinnison 1982).

1981 - Five samples of surface soil (upper inch) were collected from the site of the proposed Puget Sound Power and Light Company nuclear power generating station $2 \mathrm{mi}$ west of the Wye Barricade. Samples were collected and analyzed in the same manner as routine environmental monitoring samples. Concentrations of ${ }^{90} \mathrm{Sr},{ }^{137} \mathrm{Cs}$, and $239,240 \mathrm{Pu}$ in the soil samples appeared to typical for the general area and reflected the deposition of worldwide fallout. Results are surmarized in Figure C.6 of Appendix C.

1980 - A special study was conducted to compare ${ }^{241}$ Am concentrations in soil samples collected near the PUREX plant with samples collected off site at a distant upwind location near the town of Moxee, Washington. Soil samples consisted of the upper 0.4-in. layer, the next 0.6-in. layer, or a single sample of the upper $1.0 \mathrm{in}$. of surface soil. The study concluded that the concentrations of ${ }^{241}$ Am in samples of surface soil were very low at both sampling sites, but were slightly higher near the PUREX plant, as compared to the offsite location. More precise estimates of ${ }^{241}$ Am concentrations were noted when surface soil samples included soil crust material consisting of mosses and lichens than when samples were from bare soil. Results were reported in a PNL document (Price, Gilbert, and Gano 1981).

1979 - A special study was conducted to determine the ${ }^{239} \mathrm{Pu}$ and $240 \mathrm{Pu}$ isotopic content of surface soil samples (upper inch) collected on Site near the southwestern boundary of the Hanford Site. Routine sample collection and analysis procedures were used except that samples from five adjacent sampling locations (located $0.5 \mathrm{mi}$ apart) 
were combined into a single composite sample for analysis. Results indicated that samples collected from the general vicinity of the Arid Land Ecology field laboratory located on Site may have had slightly elevated levels of Hanford-derived plutonium compared to other onsite locations, but there was no evidence of a historical acute release of plutonium in a southwesterly direction from Hanford facilities. Results were reported in a PNL document (Price and Dirkes 1981).

1978 - A special study was conducted in 1978 to investigate the concentration of ${ }^{129}$ I in several types of samples collected from various locations on and off the Hanford Site. Surface soil samples (upper inch) were collected from Bend, Oregon; and Centralia, Wenatchee, and the Wooten Game Range near Dayton, Washington. Onsite samples were collected in 1978 near the old Hanford townsite. Data for soil samples collected from the Hanford Site in 1972 and 1974 were also evaluated. Levels of ${ }^{129}$ I in surface soils collected on Site in 1972, 1974, and 1978 showed a general reduction over the years. This reduction was probably related to the reduction in ${ }^{129}$ I releases from Hanford during the same time period and perhaps some downward leaching into the soil. Results were reported in a PNL document (Price et al. 1981).

1972 - A special radiation survey of the Wahluke Slope on the Hanford Site was conducted in 1972 to provide a basis for releasing the land for unrestricted public use. Thirty-eight surface soil samples (upper 1in. layer) were collected for analys is in addition to other sampling and radiation measurements. The study concluded that concentrations of ${ }^{90} \mathrm{Sr},{ }^{137} \mathrm{Cs}$, and ${ }^{239,240} \mathrm{Pu}$ in samples of surface soil were typical of regional fallout levels. A few exceptions were noted for locations along the Columbia River shoreline where activation products $\left({ }^{60} \mathrm{Co}\right.$ and ${ }^{65} \mathrm{Zn}$ ) had been deposited from reactor cooling water discharged to the River. Results were reported in a PNL document (Bramson and Corley 1972b).

1970 - Surface soil samples taken from the upper $0.5 \mathrm{in}$. of soil were collected in February 1970 at locations near the 200 Areas, at other locations within the Hanford Site boundary, and at locations near the Site perimeter downwind of Hanford. Results for $239,240 \mathrm{Pu}$ were 
variable with a difference of a factor of 2 that was noted between samples collected from the same site. Differences between aliquots from the same sample were also noted. Variability was attributed to a nonuniform distribution of radionuclides in the soils sampled, but other sources, such as analytical error, could not be ruled out. Data for $239,240 \mathrm{Pu}$ and ${ }^{137} \mathrm{Cs}$ concentrations for samples collected near the 200 Areas confirmed that localized areas had been affected by the deposition of effluents released to the atmosphere. Some soil samples contained slightly elevated concentrations of plutonium, as indicated by calculated plutonium to cesium ratios. Data for samples collected elsewhere, either on Site or off Site, provided plutonium to cesium ratios indicative of worldwide fallout and not Hanford operations. Results were reported as a PNL document and presented at a symposium on plutonium in the environment (Corley, Robertson, and Brauer 1972).

1970 - Samples from the upper 0.5-in. layer of soil were collected for another study conducted in February and March 1970, at onsite and offsite locations. The samples were analyzed for $239,240 \mathrm{Pu}$. Several samples were collected at some locations and analyzed separately. At other locations, a single sample was collected and several aliquots from it were analyzed separately. The results demonstrated that a large degree of variability could occur between separate samples or between aliquots of the same sample. Differences of approximately a factor of 2 were observed, and one pair of sample results differed by a factor of 20 . Results are summarized in Figure C.7 of Appendix C.

1960 - A radiological background study was conducted at the Plutonium Recycle Test Reactor site in the 300 area before startup of the facility. The study concluded that concentrations of ${ }^{137} \mathrm{Cs}$, other garma-emitting radionuclides, and uranium in surface soil samples (unspecified depths) collected at 12 locations within or near the 300 Area were consistent with sample results from other uncontaminated locations on the Hanford Site. Results were reported in a Hanford document (Williams 1960).

1957 - A special study was conducted to determine the contribution of ${ }^{90} \mathrm{Sr}$ in the environment from the deposition of effluents released to the 
atmosphere at Hanford. Samples were collected in 1956 and 1957 from onsite locations and from offsite locations as far away as Lewiston, Idaho; Davenport, Washington; and Hood River, Oregon. Surface soil samples (upper $0.5 \mathrm{in.)}$ and other types of samples were collected and analyzed. No evidence was obtained to indicate a significant deposition of ${ }^{90} \mathrm{Sr}$ in the environs from Hanford operations (i.e., deposition from Hanford was not measurable above the levels associated with worldwide fallout from nuclear weapons testing). Results were reported in a Hanford document (Silker 1958). 



\section{CONCLUSIONS OF REVIEW AND RESULTS}

A review of the environmental monitoring data base, historical records, and Hanford documents was conducted to identify data on the concentrations of ${ }^{90} \mathrm{Sr},{ }^{129} \mathrm{I},{ }^{137} \mathrm{Cs},{ }^{238} \mathrm{Pu}, 239,240 \mathrm{Pu}$ (and the individual isotopes of plutonium), ${ }^{241} \mathrm{Am}$, and uranium in soil samples collected from the Hanford Site and the local environs over the past 30 years. Most of the data were found in published annual environmental monitoring reports. Results from special-purpose studies are either referenced as published reports or summarized in Appendix $C$.

A rigorous evaluation of results was not conducted, but several observations were made with respect to the reasons cited (page 3) for the routine collection and analysis of surface soil samples for environmental monitoring.

1. Discover obvious geographical patterns of contamination. Results from soil samples collected from the offsite environs over the years have not shown gross contamination from operations at Hanford or patterns of extensive contamination. Results on the ratios of ${ }^{240} \mathrm{Pu}$ to ${ }^{239} \mathrm{Pu}$ indicated the presence of some Hanford-derived plutonium in samples collected off Site near the downwind Site boundary and near Benton City.

2. Compare results from onsite locations with those from around the Hanford Site perimeter and with distant locations. Samples collected near some onsite facilities contained traces of contamination from past operations when compared to perimeter and distant sampling locations. Radionuclides most frequently identified at enhanced levels were ${ }^{90} \mathrm{Sr},{ }^{137} \mathrm{Cs}$, and $239,240 \mathrm{Pu}$.

3. Identify trends or the gradual buildup of contamination in surface soils. No documents or data were found that indicated trends or the gradual buildup of contamination. However, slight trends would be difficult to identify because of the wide variability noted in many of the results reported.

4. Monitor areas of known contamination for indications of a change of status. Several areas of known soil contamination have been sampled 
routinely since 1971 . Results did not show a measurable reduction in the levels of contamination because of transport of materials away from the contaminated areas.

5. Test the data for unusual results that may reveal previously undetected releases of radioactive materials during Site operations. All soil samples for environmental monitoring were reported to contain some radioactive materials, but there was evidence that most of the radioactive materials were from the deposition of worldwide fallout from testing nuclear weapons in the atmosphere. No previously undetected releases of radioactive materials have been revealed by soil sampling.

Soil sampling results have been used to identify the impact from specific operations; for example, plutonium contamination near the 200 West Area and the deposition of activation products along the Columbia River shoreline from reactor cooling water. However, there are limitations to other types of uses of soil sample data. Radionuclide concentration data from routinely collected soil samples have not been useful to quantify an offsite impact from Hanford operations because of the ubiquitous distribution of worldwide fallout and the difficulty of identifying small amounts of Hanford-derived materials in the presence of fallout. No practical way is currently known to separate Hanford-derived materials from fallout when the same radionuclide is present in both sources. The mass spectrographic analysis for plutonium has been used, but it is not a method suitable for routine analysis.

Another limitation on the use of soil sampling data is the variability of results. Analytical results for aliquots from an individual sample or for multiple samples collected at the same location sometimes showed a wide degree of variability. A factor of $2(200 \%)$ was not unusual.

The last limitation concerns estimating the total amount of radioactive materials released to the atmosphere from Hanford operations and deposited onto soil. Unknown and different times of release and deposition, and the different rates of radioactive decay for each radionuclide would preclude the use of soil concentration data to estimate a total release. However, the total amount of a given radionuclide currently present in the soil could be estimated by using appropriate sampling techniques. 


\section{LITERATURE CITED}

Bramson, P. E., and J. P. Corley. 1972a. Environmental Status of the Hanford Reservation for 1971. BNWL-B-228, Pacific Northwest Laboratory, Richland, Washington.

Bramson, P. E., and J. P. Corley. 1972b. Radiation Survey of Wahluke Slope. BNWL-B-237, Pacific Northwest Laboratory, Richland, Washington

Bramson, P. E., J. P. Corley, and W. L. Nees, 1973. Environmental Status of the Hanford Reservation for 1972. BNWL-B-278, Pacific Northwest Laboratory, Richland, Washington.

Corley, J. P., D. M. Robertson, and F. P. Brauer. 1972. Plutonium in Surface Soil in the Hanford Plant Environs. PNL-SA-3967, Pacific Northwest Laboratory, Richland, Washington.

Jaquish, R. E., and P. J. Mitchell, eds. 1988. Environmental Monitoring at Hanford for 1987. PNL-6464, Pacific Northwest Laboratory, Richland, Washington.

Miller, M. L., J. J. Fix, and P. E. Bramson. 1977. Radiochemical Analyses of Soil and Veqetation Samples Taken from the Hanford Environs, 1971-1976. BNWL-2249, Pacific Northwest Laboratory, Richland, Washington.

Nees, W. L., and J. P. Corley, 1975. Environmental Status of the Hanford Reservation for 1973. BNwL-B-336, Pacific Northwest Laboratory, Richland, Washington.

Pacific Northwest Laboratory (PNL). 1987. Environmental Monitoring at Hanford for 1986. PNL-6120, Pacific Northwest Laboratory, Richland, Washington.

Price, K. R., ed. 1986. Environmental Monitoring at Hanford for 1985 . PNL-5817, Pacific Northwest Laboratory, Richland, Washington.

Price, K. R., and R. L. Dirkes, 1981. Plutonjum in Surface Soil Near the Southwestern Boundary of the Hanford Project. PNL-3647, Pacific Northwest Laboratory, Richland, Washington.

Price, K. R., and R. R. Kinnison. 1982. Uranium and Other Heavy Metals in Soil and Veqetation from the Hanford Environs. PNL-4466, Pacific Northwest Laboratory, Richland, Washington.

Price, K. R., R. 0. Gilbert, and K. A. Gano. 1981. Americium-241 in Surface Soil Associated with the Hanford Site and Vicinity. PNL-3731, Pacific Northwest Laboratory, Richland, Washington. 
Price, K. R., L. L. Cadwel1, R. G. Schreckhise, and F. P. Brauer. 1981. Iodine-129 in Forage and Deer on the Hanford Site and Other Pacific Northwest Locations. PNL-3357, Pacific Northwest Laboratory, Richland, Washington.

Silker, W. B. 1958. Strontium-90 Concentrations in the Hanford Environs. HW-55117, Hanford Atomic Products Operation, Richland, Washington.

Sula, M., and K. R. Price. 1983. PUREX Environmental Radiological Surveillance Preoperational and 0perational Support Program Conducted by Pacific Northwest Laboratory. PNL-4822, Pacific Northwest Laboratory, Richland, Washington.

Williams, L. D. 1960. Radjological Background Study of the Plutonium Recycle Test Reactor Site August-0ctober 1960. HW-68082, Hanford Atomic Products Operation, Richland, Washington. 


\section{BIBLIOGRAPHY}

ONSITE ENVIRONMENTAL MONITORING REPORTS, 1972 THROUGH 1984(a)

Bramson, P. E., and J. P. Corley. 1972. Environmental Status of the Hanford Reservation for 1971. BNWL-B-228, Pacific Northwest Laboratory, Richland, Washington.

Bramson, P. E., J. P. Corley, and W. L. Nees. 1973. Environmental Status of the Hanford Reservation for CY-1972. BNWL-B-278, Pacific Northwest Laboratory, Richland, Washington.

Fix, J. J. 1975. Environmental Status of the Hanford Reservation for CY1974. BNWL-B-429, Pacific Northwest Laboratory, Richland, Washington.

Fix, J. J., P. J. Blumer, and P. E. Bramson. 1977. Environmental Status of the Hanford Site for CY-1976. BNWL-2246, Pacific Northwest Laboratory, Richland, Washington.

Fix, J. J., D. R. Speer, and P. J. Blumer. 1976. Environmental Status of the Hanford Site for CY-1975. BNWL-B-477, Pacific Northwest Laboratory, Richland, Washington.

Houston, J. R., and P. J. Blumer. 1978. Environmental Status of the Hanford Site for CY-1.977. PNL-2677, Pacific Northwest Laboratory, Richland, Washington.

Houston, J. R., and P. J. Blumer. 1979. Environmental Status of the Hanford Site for CY-1978. PNL-2933, Pacific Northwest Laboratory, Richland, Washington.

Houston, J. R., and P. J. Blumer. 1980. Environmental Status of the Hanford Site for CY-1979. PNL-3284, Pacific Northwest Laboratory, Richland, Washington.

Nees, W. L., and J. P. Corley. 1975. Environmental Status of the Hanford Reservation for $\mathrm{CY}-1973$. BNWL-B-336, Pacific Northwest Laboratory, Richland, Washington.

Price, K. R., P. J. Blumer, J. M. V. Carlile, R. L. Dirkes, and M. S. Trevathan. 1984. Environmental Status of the Hanford Site for $\mathrm{CY}-1983$. PNL-5039, Pacific Northwest Laboratory, Richland, Washington.

Sula, M. J., P. J. Blumer, and R. L. Dirkes. 1981. Environmental Status of the Hanford Site for CY-1980. PNL-3729, Pacific Northwest Laboratory, Richland, Washington.

(a) Onsite reports were discontinued in 1985. 
Sula, M. J., P. J. Blumer, and R. L. Dirkes. 1982. Environmental Status of the Hanford Site for CY-1981. PNL-4212, Pacific Northwest Laboratory, Richland, washington.

Sula, M. J., P. J. Blumer, R. L. Dirkes, and J. M. V. Carlile. 1983. Environmental Status of the Hanford Site for CY-1982. PNL-4658, Pacific Northwest Laboratory, Richland, Washington.

\section{OFFSITE ENVIRONMENTAL MONITORING REPORTS, 1972. THROUGH 1986}

Blumer, P. J., J. J. Fix, and D. R. Speer. 1976. Environmental Surveillance at Hanford for CY-1975 Data. BNWL-1980, Pacific Northwest Laboratory, Richland, Washington.

Bramson, P. E., and J. P. Corley. 1972. Environmental Surveillance at Hanford for CY-1971. BNWL-1683, Pacific Northwest Laboratory, Richland, Washington.

Bramson, P. E., and J. P. Corley. 1972. Environmental Surveillance at Hanford for CY-1971 Data. BNWL-1683-ADD, Pacific Northwest Laboratory, Richland, Washington.

Bramson, P. E., and J. P. Corley. 1973. Environmental Surveillance at Hanford for CY-1972. BNWL-1727, Pacific Northwest Laboratory, Richland, Washington.

Bramson, P. E., and J. P. Corley. 1973. Environmental Surveillance at Hanford for CY-1972 Data. BNWL-1727-ADD, Pacific Northwest Laboratory, Richland, Washington.

Fix, J. J. 1976. Environmental Surveillance at Hanford for CY-1974. BNWL1910, Pacific Northwest Laboratory, Richland, Washington.

Fix, J. J., and P. J. Blumer. 1975. Environmental Surveillance at Hanford for CY-1974 Data. BNWL-1910-ADD, Pacific Northwest Laboratory, Richland, Washington.

Fix, J. J., P. J. Blumer, G. R. Hoenes, and P. E. Bramson. 1977. Environmental Surveillance at Hanford for CY-1976. BNWL-2142, Pacific Northwest Laboratory, Richland, Washington.

Houston, J. R., and P. J. Blumer. 1978. Environmental Surveillance at Hanford for CY-1977. PNL-2614, Pacific Northwest Laboratory, Richland, Washington.

Houston, J. R., and P. J. Blumer. 1979. Environmental Surveillance at Hanford for CY-1978. PNL-2932, Pacific Northwest Laboratory, Richland, Washington. 
Houston, J. R., and P. J. Blumer. 1980. Environmental Surveillance at Hanford for CY-1979. PNL-3283, Pacific Northwest Laboratory, Richland, Washington.

Jaquish, R. E., and P. J. Mitchel1, eds. 1988. Environmental Monitoring at Hanford for 1987. PNL-6464, Richland, Washington.

Nees, W. L., and J. P. Corley. 1974. Environmental Surveillance at Hanford for CY-1973. BNWL-1811, Pacific Northwest Laboratory, Richland, Washington.

Nees, W. L., and J. P. Corley. 1974. Environmental Surveillance at Hanford for CY-1973 Data. BNWL-1811-ADD, Pacific Northwest Laboratory, Richland, Washington.

Pacific Northwest Laboratory (PNL). 1987. Environmental Monitoring at Hanford for 1986. PNL-6120, Pacific Northwest Laboratory, Richland, Washington.

Price, K. R., J. M. V. Carlile, R. L. Dirkes, and M. S. Trevathan. 1984. Environmental Surveillance at Hanford for CY-1983. PNL-5038, Pacific Northwest Laboratory, Richland, Washington.

Price, K. R., J. M. V. Carlile, R. L. Dirkes, R. E. Jaquish, M. S. Trevathan, and R. K. Woodruff. 1985. Environmental Monitoring at Hanford for 1984. PNL-5407, Pacific Northwest Laboratory, Richland, Washington.

Price, K. R. (ed). 1986. Environmental Monitoring at Hanford for 1985. PNL-5817, Pacific Northwest Laboratory, Richland, Washington.

Speer, D. R., J. J. Fix, and P. J. Blumer. 1976. Environmental Surveillance at Hanford for $\mathrm{CY}-1975$. BNWL-1979 (Rev.), Pacific Northwest Laboratory, Richland, Washington.

Sula, M. J., and P. J. Blumer. 1981. Environmental Surveillance at Hanford for CY-1980. PNL-3728, Pacific Northwest Laboratory, Richland, Washington.

Sula, M. J., W. D. McCormack, R. L. Dirkes, K. R. Price, and P. A. Eddy. 1982. Environmental Surveillance at Hanford for CY-1981. PNL-4211, Pacific Northwest Laboratory, Richland, Washington.

Sula, M. J., J. M. V. Carlile, K. R. Price, and W. D. McCormack. 1983. Environmental Surveillance at Hanford for CY-1981. PNL-4657, Pacific Northwest Laboratory, Richland, Washington. 

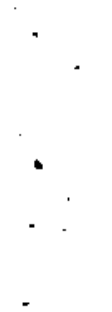


\section{APPENDIX A}

ONSITE ROUTINE SOIL SAMPLING LOCATIONS AND RESULTS 
TABLE A.1. Onsite Routine Soil Sampling Locations, 1971 through 1987

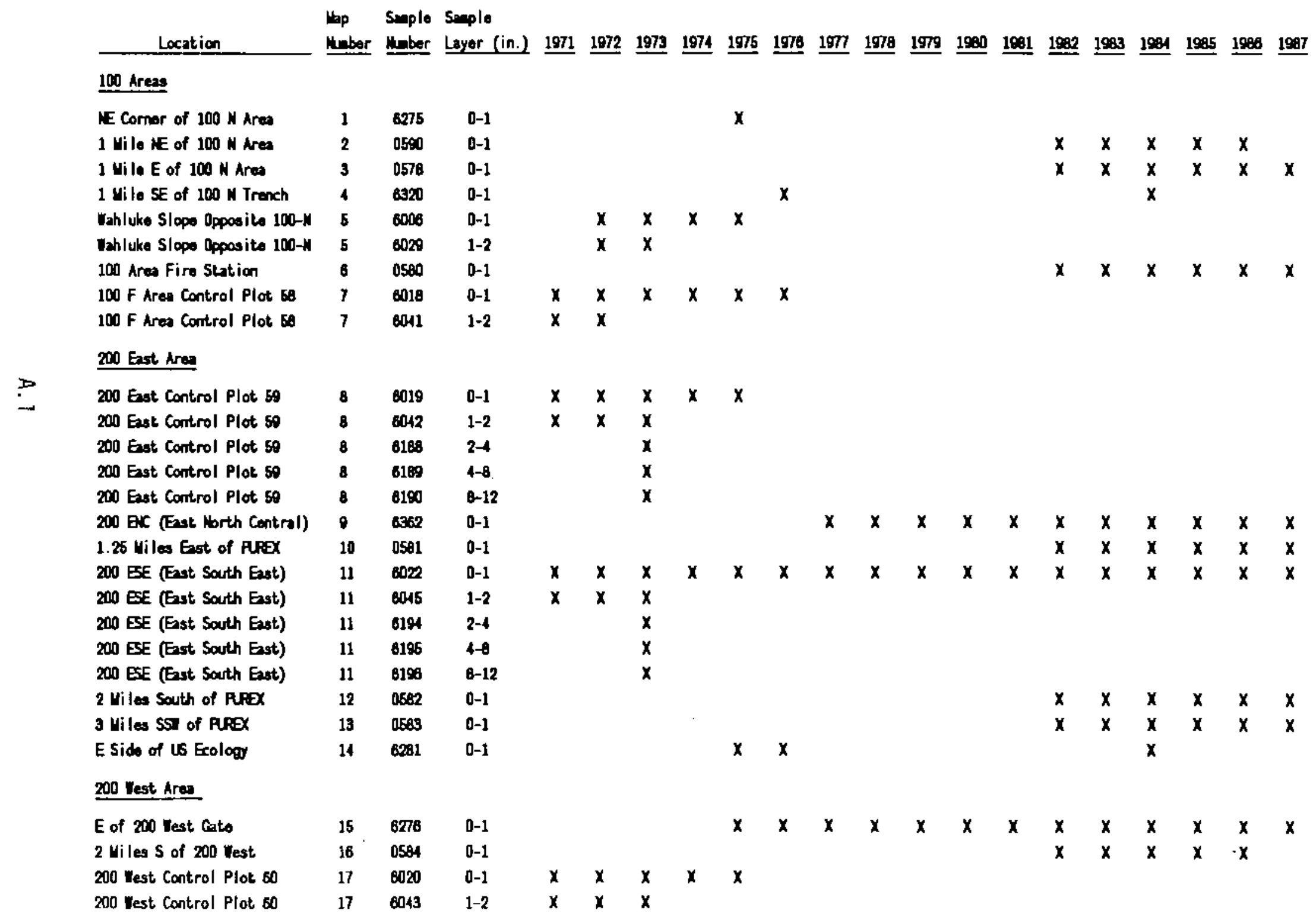


IABLE A.1. (contd)

Location

Hap Saplo Sasple

200 West Area (contd)

200 Vest control Plot 60

200 liest Control Plot 80

200 Mest Control Plot 80

$\begin{array}{lll}17 & 0191 & 2-4 \\ 17 & 0192 & 4-8\end{array}$

Other 200 Area Locations

200 Area Fire Station

200 Area Fire Station

Gable Pond Control Plot 40

Inter. 45 and Any Loop PA.

Any Loop Ad. Contl. Plot 40

Highray 240 Control Plot 54

Highray 240 Control Plot 6

300 Area

Horth of 300 Area

North Center 300 Area Fence

300 Area South Bte

South of 300 Aren

391 Bldg. Control Plot 60

331 Bldg. Control Plot EO

400 Area and Vicinity

\section{Wye Berricade}

Iye Barricado

Tye terricade (RC Dapl. A)

Yyo Barricado (R Depl. B)

1 Hile $N$ of Uipss to. 2

1 vilo $S$ of uPAS to. 2

FFIF Control Plot $B 2$

FIF control Plot 62

D.5 vile NE of FIF

SE Side of FIF

$\begin{array}{lll}20 & 6018 & 0-1 \\ 29 & 6039 & 1-2 \\ 29 & 0818 & 0-1 \\ 28 & 0817 & 0-1 \\ 29 & 6324 & 0-1 \\ 29 & 6325 & 0-1 \\ 30 & 6015 & 0-1 \\ 30 & 8039 & 1-2 \\ 31 & 8292 & 0-1 \\ 32 & 627 & 0-1\end{array}$

\begin{tabular}{|c|c|c|c|c|c|c|c|}
\hline 10 & 0021 & $0-1$ & $x$ & $x$ & $x$ & & \\
\hline 18 & 6044 & $1-2$ & $x$ & $x$ & & & \\
\hline 19 & 8321 & $0-1$ & & & & & \\
\hline 20 & 8223 & $0-1$ & & & & $x$ & $x$ \\
\hline 21 & 6228 & $0-1$ & & & & $x$ & \\
\hline 22 & 6002 & $0-1$ & $x$ & $x$ & $x$ & $x$ & \\
\hline 22 & 6025 & $1-2$ & $x$ & $x$ & & & \\
\hline
\end{tabular}

\begin{tabular}{|c|c|c|c|c|c|}
\hline $\mathbf{2}$ & 6322 & $0-1$ & & & \\
\hline 24 & 6279 & $0-1$ & & & \\
\hline 25 & 6222 & $0-1$ & & & \\
\hline 28 & 6323 & $0-1$ & & & \\
\hline 27 & 8014 & $0-1$ & $x$ & $x$ & $\mathbf{x}$ \\
\hline 27 & BD97 & $1-2$ & $x$ & $x$ & \\
\hline
\end{tabular}

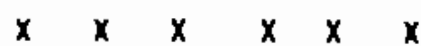

$x \quad x \quad x \quad x$

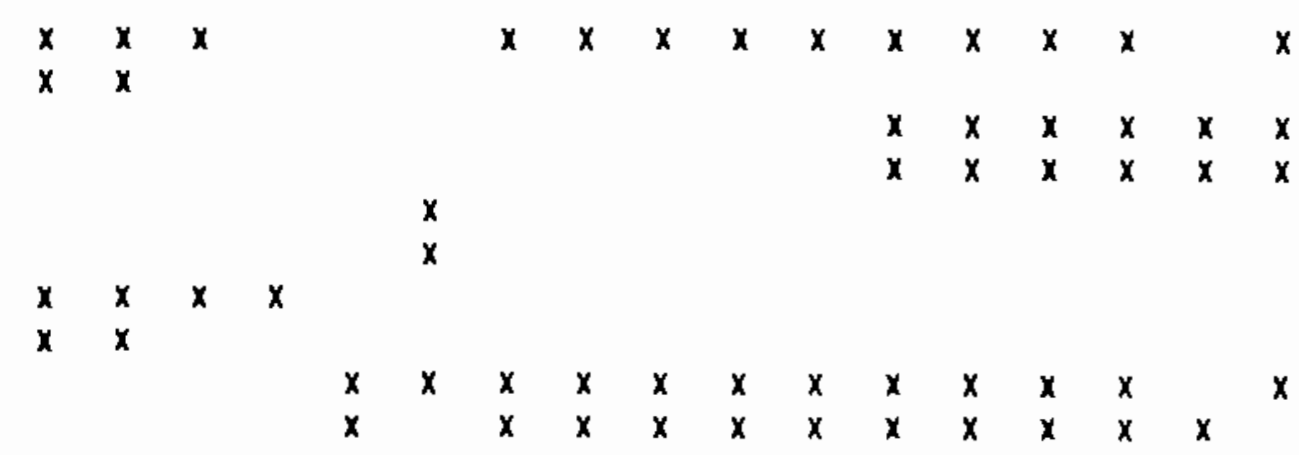

$x$

$\begin{array}{llllllllllll}x & x & x & x & x & x & x & x & x & x & & x \\ & x & x & x & x & x & x & x & x & x & x & \end{array}$


TABLE A.1. (contd)

\begin{tabular}{|c|c|c|c|c|c|c|c|c|c|c|c|c|c|c|c|c|c|c|c|c|}
\hline Location & $\begin{array}{l}\text { Nap } \\
\text { Number }\end{array}$ & $\begin{array}{l}\text { Sample } \\
\text { Nuber }\end{array}$ & $\begin{array}{l}\text { Sanple } \\
\text { Layer (in.) }\end{array}$ & 1971 & 1972 & 1973 & 1974 & 1975 & 1978 & 1977 & 1978 & 1970 & 1980 & 1991 & 1982 & 1983 & 1994 & 1985 & 1986 & 1997 \\
\hline \multicolumn{21}{|l|}{ Other Onsito Locations } \\
\hline N End of Vernita Bridgo & 33 & 6005 & $0-1$ & $\mathbf{x}$ & $\mathbf{x}$ & $x$ & $x$ & & & & & & & & $x$ & $\mathbf{x}$ & $\mathbf{x}$ & $\mathbf{x}$ & $\mathbf{x}$ & \\
\hline N End of Vernita Bridgo & 33 & 6028 & $1-2$ & $x$ & $x$ & & & & & & & & & & & & & & & \\
\hline Hanford Tomsite & 34 & B017 & $0-1$ & $x$ & $x$ & $x$ & $\because$ & $x$ & $\mathbf{x}$ & $\mathbf{x}$ & $x$ & $\mathbf{x}$ & $x$ & $\mathbf{x}$ & $x$ & $x$ & $x$ & $x$ & & $x$ \\
\hline Hanford Tounsito & 34 & 6040 & $1-2$ & $x$ & $x$ & & & & & & & & & & & & & & & \\
\hline Prosser Barricado & 35 & 6225 & $0-1$ & & & & $x$ & $x$ & & $x$ & $x$ & $x$ & $x$ & $x$ & $x$ & $x$ & $x$ & $x$ & $x$ & \\
\hline EC (Enor. Roloc. Center) & 36 & 6001 & $0-1$ & $x$ & $x$ & $\mathbf{x}$ & $\mathbf{x}$ & & & & & & & & & & · & & & \\
\hline EC (Evor. Roloc. Center) & 38 & 6024 & $1-2$ & $x$ & $x$ & & & & & & & & & & & & & & & \\
\hline E of Arid Land Ecol. Lab & 37 & 6278 & $0-1$ & & & & & $x$ & $x$ & $\mathbf{x}$ & $x$ & $x$ & $\mathbf{x}$ & $x$ & $x$ & $x$ & $x$ & $x$ & & $x$ \\
\hline Battlesnake Springs & 38 & 6003 & $0-1$ & $x$ & $x$ & $x$ & $x$ & & & & & & & & $x$ & $x$ & $x$ & $x$ & $x$ & $x$ \\
\hline Rattlesnake Springs & 38 & 6026 & $1-2$ & $x$ & $x$ & & & & & & & & & & & & & & & \\
\hline Yakina Barricade & 39 & 6004 & $0-1$ & $x$ & $x$ & $x$ & $x$ & $x$ & $x$ & $x$ & $x$ & $x$ & $x$ & $x$ & $x$ & $x$ & $x$ & $x$ & & $x$ \\
\hline Yakima Barricade & 39 & 6027 & $1-2$ & $x$ & $x$ & & a & & & & & & & & & & & & & \\
\hline
\end{tabular}




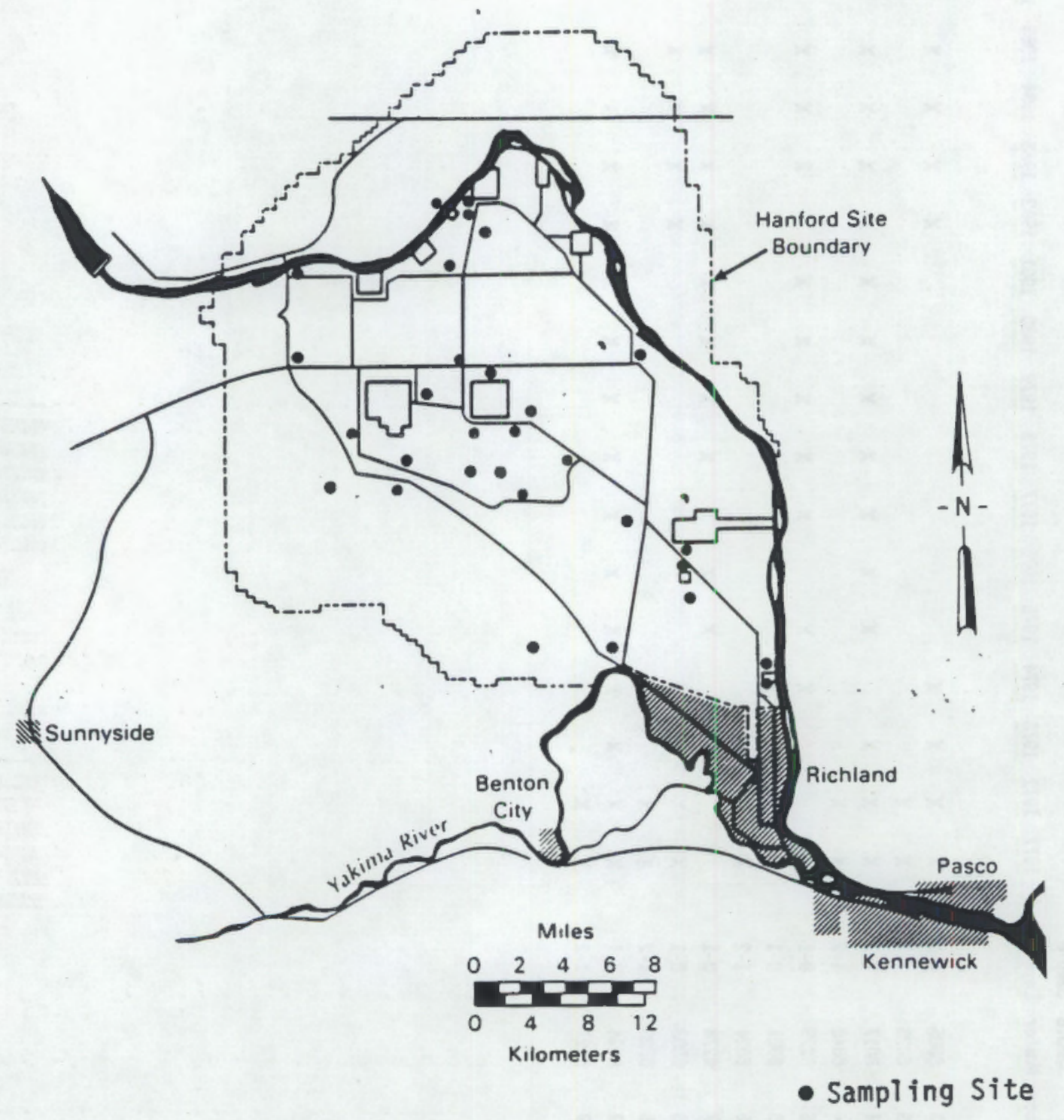

FIGURE A.1. Onsite Routine Soil Sampling Locations, 1971 to 1987 
TABLE A.2. Strontium-90 Concentrations in Soll at Onsite Locations

100 Areas

NE Corner of $100 \mathrm{~N}$ Area

6275 Depth $0-1$ in.

\begin{tabular}{llll} 
Sample & Dup. & Result & $+/-2$ \\
Date & No. & pCi/g & Std. Dev. \\
\hdashline $08 / 20 / 75$ & & $0.2110 \mathrm{E}+01$ & $0.000 \mathrm{E}+00$
\end{tabular}

1 Mile NE of $100 \mathrm{~N}$ Area

0590 Depth 0-1 in.

\begin{tabular}{llll} 
Sample & Dup. & Result & $+/-2$ \\
Date & No. & pCi/g & std. Dev. \\
\hline $04 / 30 / 86$ & & $0.2390 \mathrm{E}+00$ & $0.100 \mathrm{E}-01$ \\
$06 / 24 / 85$ & & $0.2750 \mathrm{D}+00$ & $0.241 \mathrm{E}-01$ \\
$05 / 17 / 84$ & & $0.2940 \mathrm{E}+00$ & $0.166 \mathrm{E}-01$ \\
$08 / 05 / 83$ & & $0.7000 \mathrm{E}+00$ & $0.227 \mathrm{E}-01$ \\
$08 / 24 / 82$ & & $0.1980 \mathrm{E}+00$ & $0.324 \mathrm{E}-01$
\end{tabular}

1 Mile $E$ of $100 \mathrm{~N}$ Area

0578 Depth $0-1$ in.

\begin{tabular}{|c|c|c|c|}
\hline $\begin{array}{l}\text { Sample } \\
\text { Date }\end{array}$ & $\begin{array}{l}\text { Dup. } \\
\text { No. }\end{array}$ & $\begin{array}{l}\text { Regult } \\
\text { pCi'/g }\end{array}$ & $\begin{array}{l}+/-2 \\
\text { std. Dev. }\end{array}$ \\
\hline $\begin{array}{l}-2 / 27 / 87 \\
04 / 29 / 87 \\
04 / 30 / 86 \\
06 / 24 / 85 \\
05 / 17 / 84 \\
08 / 11 / 83 \\
08 / 24 / 82\end{array}$ & & $\begin{array}{l}0.3070 \mathrm{E}+00 \\
0.2190 \mathrm{E}+00 \\
0.4390 \mathrm{E}+00 \\
0.2170 \mathrm{E}+00 \\
0.8520 \mathrm{E}+00 \\
0.1530 \mathrm{E}+00\end{array}$ & $\begin{array}{l}--135 \mathrm{E}-01 \\
0.1301 \\
0.103 \mathrm{E}-01 \\
0.299 \mathrm{E}-01 \\
0.117 \mathrm{E}-01 \\
0.25 \mathrm{E}-01 \\
0.5 \mathrm{E}-01\end{array}$ \\
\hline
\end{tabular}

1 Mile SE of $100 \mathrm{~N}$ Trench

\begin{tabular}{llll}
6320 & Depth $0-1$ in. & Sr-90 \\
Sample & Dup. & Regult & $+/ 2$ \\
Date & No. & pCi $/ g$ & Std. Dev. \\
\hdashline $00 / 03 / 84$ & & $0.3230 E+00$ & $0.190 E-01$ \\
$08 / 11 / 76$ & & $0.2210 E+00$ & $0.000 E+00$
\end{tabular}

Wahluke slope Opposite 100-N

$\begin{array}{llll}6006 & \text { Depth } 0-1 \text { in. } & \text { Sr-90 } \\ & & & \\ \text { Sample } & \text { Dup. } & \text { Regult } & +/-2 \\ \text { Date } & \text { No. } & \text { pCi/g } & \text { std. Dev. } \\ -09 / 23 / 74 & & 0.2520 E-01 & 0.000 E+00 \\ 10 / 25 / 73 & & 0.3130 E+00 & 0.520 E-02 \\ 10 / 25 / 72 & & 0.3630 E-01 & 0.000 E+00 \\ 09 / 29 / 71 & & 0.1280 E+00 & 0.000 E+00\end{array}$

$\begin{array}{llll}\text { Wahluke slope Opposite 100-N } & \text { S } \\ 6029 & \text { Depth } 1-2 \text { in. } & \text { Sr-90 } \\ \text { Sample } & \text { Dup. Regult } & +/-2 \\ \text { Date } & \text { No. } & \text { pCi/g } & \text { std. Dev. } \\ -10 / 25 / 72 & & 0.4570 E-01 & 0.000 E+00 \\ 09 / 29 / 71 & & 0.1560 \mathrm{E}+00 & 0.000 \mathrm{E}+00\end{array}$

\begin{tabular}{llll}
100 Area & Fire Station \\
0580 & Depth $0-1$ in. & sr-90 \\
Sample & Dup. Result & $+/-2$ \\
Date & No. & DCi/g & std. Dev. \\
\hline $04 / 29 / 87$ & & $0.3310 E+00$ & $0.123 \mathrm{E}-01$ \\
$04 / 30 / 86$ & $0.3430 \mathrm{E}+00$ & $0.106 \mathrm{E}-01$ \\
$05 / 30 / 85$ & $0.5650 \mathrm{E}+00$ & $0.212 \mathrm{E}-01$ \\
$05 / 17 / 84$ & $0.4530 \mathrm{E}+00$ & $0.237 \mathrm{E}-01$ \\
$08 / 05 / 83$ & $0.1670 \mathrm{E}+01$ & $0.327 \mathrm{E}-01$ \\
$0 \mathrm{~B} / 24 / \mathrm{B2}$ & $0.2810 \mathrm{E}+00$ & $0.352 \mathrm{E}-01$
\end{tabular}

100 F Area Control Plot 58

6018 Depth $0-1$ in. sr-90

Sample Dup, Result $+/-2$

Date No. $\mathrm{DCi} / \mathrm{g}$ std. Dev.

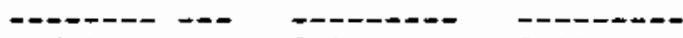

$08 / 11 / 76 \quad 0.1890 E+00 \quad 0.000 E+00$

$08 / 18 / 75 \quad 0.3300 E+00 \quad 0.000 E+00$

$09 / 24 / 74 \quad 0.3170 E+00 \quad 0.000 E+00$

$10 / 25 / 73 \quad 0.2850 E+00 \quad 0.450 E-02$

$10 / 25 / 72 \quad 0.2850 \mathrm{E}+00 \quad 0.000 \mathrm{E}+00$

$09 / 30 / 71 \quad 0.4090 \mathrm{E}+00 \quad 0.000 \mathrm{E}+00$

$\begin{array}{llll}100 \text { F Area Control Plot } 58 & \\ 6041 & \text { Depth } 2-2 \text { in. } & \text { Sr-90 } \\ & & & \\ \text { Sample } & \text { Dup. Result } & +/-2 \\ \text { Date } & \text { No. } & \text { pCi/g } & \text { std. Dev. } \\ -10 / 25 / 72 & & 0.1040 E+00 & 0.000 E+00 \\ 09 / 30 / 71 & & 0.1000 E-02 & 0.000 E+00\end{array}$


TABLE A.2. (contd)

200 East Area

\begin{tabular}{|c|c|c|c|}
\hline $\begin{array}{l}200 \text { East } \\
6019\end{array}$ & $\begin{array}{l}\text { Contr } \\
\text { Depth }\end{array}$ & $0-1$ in. & $\operatorname{Sr}-90$ \\
\hline $\begin{array}{l}\text { Sample } \\
\text { Date }\end{array}$ & $\begin{array}{l}\text { Dup. } \\
\text { No. }\end{array}$ & $\begin{array}{l}\text { Result } \\
\text { pci/g }\end{array}$ & $\begin{array}{l}+/-{ }^{2} \\
\text { Std. Dev. }\end{array}$ \\
\hline $\begin{array}{l}0---1 / 18 / 75 \\
08 / 18 / 24 / 74 \\
09 / 25 / 73 \\
10 / 25 / 72 \\
09 / 30 / 71\end{array}$ & $--\infty$ & $\begin{array}{l}0.2810 \mathrm{E}+00 \\
0.7600 \mathrm{E}+00 \\
0.1240 \mathrm{E}+00 \\
0.2100 \mathrm{E}+00 \\
0.1020 \mathrm{E}+00\end{array}$ & $\begin{array}{l}0.000 E+00 \\
0.000 E+00 \\
0.360 E-02 \\
0.000 E+00 \\
0.000 E+00\end{array}$ \\
\hline
\end{tabular}

\begin{tabular}{|c|c|c|c|}
\hline 6042 & Depth & $1-2$ in. & SI -90 \\
\hline $\begin{array}{l}\text { Sample } \\
\text { Date }\end{array}$ & $\begin{array}{l}\text { Dup. } \\
\text { No. }\end{array}$ & $\begin{array}{l}\text { Regult } \\
\mathrm{pCi} / \mathrm{g}\end{array}$ & $\begin{array}{l}+/-{ }^{2} \\
\text { std. Dev. }\end{array}$ \\
\hline 73 & $-\infty$ & $\begin{array}{l}0.8260 \mathrm{E}-01 \\
0.5210 \mathrm{E}-01\end{array}$ & $\begin{array}{l}0.270 \mathrm{E}-02 \\
0.000 \mathrm{E}+00\end{array}$ \\
\hline
\end{tabular}

\begin{tabular}{|c|c|c|c|}
\hline $\begin{array}{l}200 \text { East } \\
6188\end{array}$ & $\begin{array}{l}\text { Contr } \\
\text { Depth }\end{array}$ & $2-4$ in. & SI -90 \\
\hline $\begin{array}{l}\text { Sample } \\
\text { Date }\end{array}$ & $\begin{array}{l}\text { Dup. } \\
\text { No. }\end{array}$ & $\begin{array}{l}\text { Result } \\
\text { pCi/g }\end{array}$ & $\begin{array}{l}+/-2 \\
\text { std. Dev. }\end{array}$ \\
\hline$\overline{10 / 25 / 73}$ & --- & $0.1640 \mathrm{E}-01$ & $0.230 \mathrm{E}-02$ \\
\hline
\end{tabular}

200 East Control Plot 59 6189 Depth 4-8 in.

$5 r-90$

\begin{tabular}{llll} 
Sample & Dup. & Result & $+/-2$ \\
Date & No. & pCi/g & std. Dev. \\
\hdashline $10 / 25 / 73$ & & $<0.0000 E+00$ & $0.230 E-02$
\end{tabular}

\begin{tabular}{|c|c|c|c|}
\hline $\begin{array}{l}200 \text { East } \\
6190\end{array}$ & Depth & 8-12 in. & Sr-90 \\
\hline $\begin{array}{l}\text { Sample } \\
\text { Date }\end{array}$ & $\begin{array}{l}\text { Dup. } \\
\text { No. }\end{array}$ & $\begin{array}{l}\text { Result } \\
\mathrm{pCi} / \mathrm{g}\end{array}$ & $\begin{array}{l}+/-2 \\
\text { std, Dev. }\end{array}$ \\
\hline $10 / 25 / 73$ & --- & $0.20308-01$ & $0.230 \mathrm{E}-02$ \\
\hline
\end{tabular}

200 ENC (East North Central)

6362 Depth $0-1$ in. Sr-90

Sample Dup. Result $t /-2$

Date No. pCi/g std, Dev.

$\overline{05 / 07 / 87}--\overline{0.1130 E+01} \cdot \overline{0.406 \mathrm{E}-01}$

$04 / 30 / 86 \quad 0.6140 \mathrm{E}+00 \quad 0.143 \mathrm{E}-01$

$05 / 31 / 85 \quad 0.1180 \mathrm{E}+01 \quad 0.275 \mathrm{E}-01$

$05 / 17 / 84 \quad 0.1960 \mathrm{E}+00 \quad 0.194 \mathrm{E}+00$

$0 B / 12 / B 3 \quad 0.2720 E+01 \quad 0.466 E-01$

$08 / 30 / 82 \quad 0.9310 \mathrm{E}+000.128 \mathrm{E}+00$

$0 \mathrm{~B} / 10 / \mathrm{B} 1 \mathrm{I} \quad 0.6300 \mathrm{E}+00 \quad 0.630 \mathrm{E}-02$

$0 \mathrm{~B} / 10 / 81 \quad 0.4520 \mathrm{E}+00 \quad 0.230 \mathrm{E}-01$

$08 / 05 / 80 \quad 0.6950 E+00 \quad 0.570 E-01$

$09 / 11 / 79 \quad 0.9540 \mathrm{E}+00 \quad 0.150 \mathrm{E}-01$

$08 / 10 / 7 \mathrm{~B} \quad 0.6980 \mathrm{E}+00 \quad 0.170 \mathrm{E}-01$

$06 / 14 / 77<0.2250 E-02 \quad 0.1508-01$

1.25 Miles East of PUREX

0581 Depth 0-1 in. Sr-90

\begin{tabular}{|c|c|c|c|}
\hline $\begin{array}{l}\text { Sample } \\
\text { Date }\end{array}$ & $\begin{array}{l}\text { Dup. } \\
\text { No. }\end{array}$ & $\begin{array}{l}\text { Result } \\
\mathrm{pCi} / \mathrm{g}\end{array}$ & $\begin{array}{l}+/-2 \\
\text { std. Dev. }\end{array}$ \\
\hline $\begin{array}{l}05 / 07 / 87 \\
04 / 30 / 86 \\
05 / 31 / 85 \\
05 / 15 / 84 \\
08 / 05 / 83 \\
08 / 24 / 82\end{array}$ & $-\sim$ & $\begin{array}{l}0.3440 \mathrm{E}+00 \\
0.3920 \mathrm{E}+00 \\
0.9000 \mathrm{E}+00 \\
0.7260 \mathrm{E}+00 \\
0.7760 \mathrm{E}+00 \\
0.3340 \mathrm{E}+00\end{array}$ & $\begin{array}{l}0.202 \mathrm{E}-01 \\
0.129 \mathrm{E}-01 \\
0.294 \mathrm{E}-01 \\
0.482 \mathrm{E}-01 \\
0.327 \mathrm{E}-01 \\
0.0 \mathrm{~B} 3 \mathrm{E}-01\end{array}$ \\
\hline
\end{tabular}


TABLE A.2. (contd)

200 Bast Area

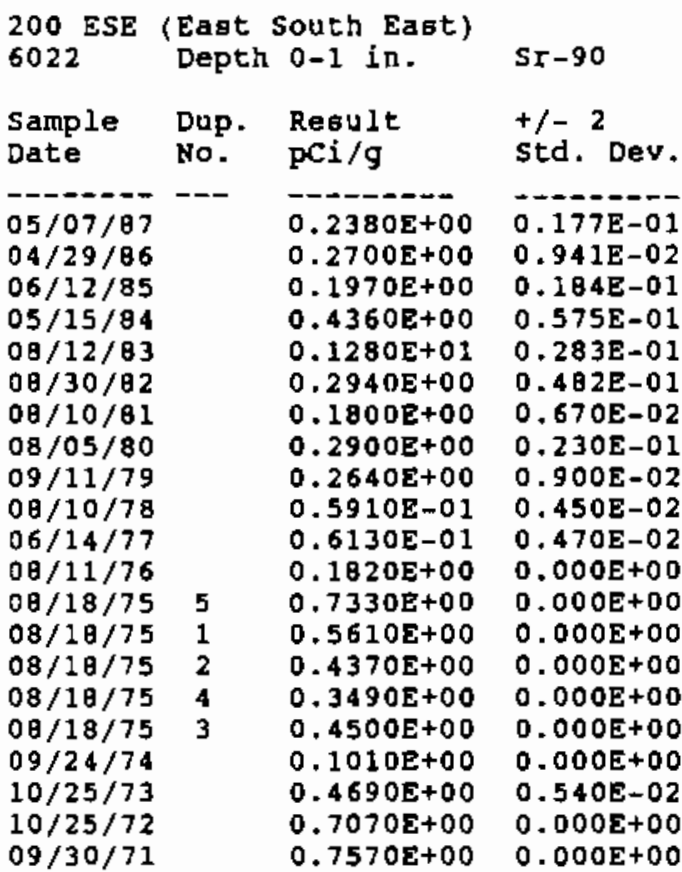

\begin{tabular}{|c|c|c|c|}
\hline 045 & Depth & 1-2 in. & Sr-90 \\
\hline $\begin{array}{l}\text { Sample } \\
\text { Date }\end{array}$ & $\begin{array}{l}\text { Dup. } \\
\text { No. }\end{array}$ & $\begin{array}{l}\text { Result } \\
\mathrm{pCi} / \mathrm{g}\end{array}$ & $\begin{array}{l}+/-2 \\
\text { std. Dev. }\end{array}$ \\
\hline $\begin{array}{l}-10 / 25 / 73 \\
10 / 25 / 72 \\
09 / 30 / 71\end{array}$ & --- & $\begin{array}{l}0.2100 \mathrm{E}+00 \\
0.2990 \mathrm{0}+00 \\
0.1220 \mathrm{E}+00\end{array}$ & $\begin{array}{l}0.360 \mathrm{E}-02 \\
0.000 \mathrm{E}+00 \\
0.000 \mathrm{E}+00\end{array}$ \\
\hline
\end{tabular}

\begin{tabular}{llll}
200 ESE & $\begin{array}{l}\text { Eagt South East) } \\
\text { Depth } 8-12 \text { in. }\end{array}$ & Sr-90 \\
Sample & Dup. Regult & $+/-2$ \\
Date & No. PCi/g & Std. Dev. \\
\hdashline $10 / 25 / 73$ & & $0.1370 \mathrm{E}-01$ & $0.230 \mathrm{E}-02$
\end{tabular}

2 Milee south of PUREX

0582 Depth $0-1$ in.

$\operatorname{Sr}-90$

Sample Dup. Result $+/-2$

Dato No. pCi/g std. Dey.

05/07/87 -- - -

$04 / 30 / 86 \quad 0.5370 \mathrm{E}+00 \quad 0.14 \mathrm{BE}-01$

$06 / 24 / 85 \quad 0.1370 E+00 \quad 0.141 E-01$

$05 / 15 / 84 \quad 0.5020 E+00 \quad 0.105 E+00$

$08 / 05 / 83 \quad 0.3790 E+00 \quad 0.200 E-01$

$08 / 24 / 82 \quad 0.1800 E+00 \quad 0.459 E-01$

\begin{tabular}{llll}
$\begin{array}{l}3 \text { Mileg } \\
\text { O583 }\end{array}$ & $\begin{array}{l}\text { SSw of PUREX } \\
\text { Depth } 0-1 \text { in. }\end{array}$ & Sr-90 \\
& & & \\
Sample & Dup. & Result & $+/-2$ \\
Date & No. & pCi/g & std. Dev. \\
\hline $05 / 07 / 87$ & & $0.2010 \mathrm{E}-01$ & $0.520 \mathrm{E}-02$ \\
$04 / 30 / 86$ & & $0.1130 \mathrm{E}+00$ & $0.662 \mathrm{E}-02$ \\
$06 / 24 / 85$ & $0.3930 \mathrm{E}+00$ & $0.227 \mathrm{E}-01$ \\
$05 / 15 / 84$ & $0.1160 \mathrm{E}+00$ & $0.504 \mathrm{E}-01$ \\
$08 / 05 / 83$ & & $0.7930 \mathrm{E}+00$ & $0.332 \mathrm{E}-01$ \\
$08 / 24 / 82$ & & $0.1300 \mathrm{E}+00$ & $0.998 \mathrm{E}-01$
\end{tabular}

\begin{tabular}{|c|c|c|c|c|c|c|c|}
\hline 6194 & Depth & $2-4$ in. & $S r-90$ & $\begin{array}{l}\text { Sample } \\
\text { Date }\end{array}$ & $\begin{array}{l}\text { Dup. } \\
\text { No. }\end{array}$ & $\begin{array}{l}\text { Result } \\
\mathrm{pCi} / \mathrm{g}\end{array}$ & $\begin{array}{l}+/-2 \\
\text { std. Dev. }\end{array}$ \\
\hline $\begin{array}{l}\text { Sample } \\
\text { Date }\end{array}$ & $\begin{array}{l}\text { Dup. } \\
\text { No. }\end{array}$ & $\begin{array}{l}\text { Result } \\
\text { pCi/g }\end{array}$ & $\begin{array}{l}+/-2 \\
\text { std. Dev. }\end{array}$ & $08 / 03 / 84$ & --- & $0.9670 \mathrm{E}-01$ & $0.116 \mathrm{E}-01$ \\
\hline $10 / 25 / 73$ & --- & $0.18608+00$ & $0.410 \mathrm{E}-02$ & $\begin{array}{l}08 / 11 / 76 \\
08 / 18 / 75\end{array}$ & & $\begin{array}{l}0.2450 \mathrm{E}+00 \\
0.5770 \mathrm{E}-01\end{array}$ & $\begin{array}{l}0.000 \mathrm{E}+00 \\
0.000 \mathrm{E}+00\end{array}$ \\
\hline
\end{tabular}

\begin{tabular}{|c|c|c|c|}
\hline $\begin{array}{l}200 \text { ESE } \\
6195\end{array}$ & Depth & 4-8 in. & Sr-90 \\
\hline $\begin{array}{l}\text { Sample } \\
\text { Date }\end{array}$ & $\begin{array}{l}\text { Dup. } \\
\text { No. }\end{array}$ & $\begin{array}{l}\text { Regult } \\
\mathrm{pCi} / \mathrm{g}\end{array}$ & $\begin{array}{l}+/-2 \\
\text { Std. Dev. }\end{array}$ \\
\hline $10 / 25 / 73$ & --- & $0.9230 \mathrm{E}-02$ & $0.230 \mathrm{E}-02$ \\
\hline
\end{tabular}


TABLE A.2. (contd)

200 West Area

$E$ of 200 West Gate

\begin{tabular}{|c|c|c|c|}
\hline 276 & Depth & $0-1$ in. & $S I-90$ \\
\hline $\begin{array}{l}\text { Sample } \\
\text { Date }\end{array}$ & $\begin{array}{l}\text { Dup. } \\
\text { No. }\end{array}$ & $\begin{array}{l}\text { Result } \\
\mathrm{pci} / \mathrm{g}\end{array}$ & $\begin{array}{l}+/-2 \\
\text { std. Dev. }\end{array}$ \\
\hline$-\infty---$ & --- & ------- & ----n--- \\
\hline $\begin{array}{l}05 / 07 / 87 \\
05 / 01 / 86 \\
05 / 31 / 85 \\
05 / 15 / 84 \\
08 / 12 / 83 \\
08 / 30 / 82 \\
08 / 10 / 81 \\
08 / 04 / 80 \\
09 / 11 / 79 \\
00 / 09 / 78 \\
06 / 14 / 77 \\
08 / 11 / 76\end{array}$ & & $\begin{array}{l}0.3800 \mathrm{E}+00 \\
0.5610 \mathrm{E}+00 \\
0.6050 \mathrm{E}+00 \\
0.3340 \mathrm{E}+00 \\
0.2560 \mathrm{E}+01 \\
0.4770 \mathrm{0}+00 \\
0.4610 \mathrm{E}+00 \\
0.8250 \mathrm{E}+00 \\
0.1110 \mathrm{E}+01 \\
0.1320 \mathrm{E}-01 \\
0.1530 \mathrm{E}+00 \\
0.2540 \mathrm{E}+00\end{array}$ & $\begin{array}{l}0.199 E-01 \\
0.143 E-01 \\
0.387 E-01 \\
0.222 E-01 \\
0.480 E-01 \\
0.105 E+00 \\
0.270 E-01 \\
0.950 E-02 \\
0.180 E-01 \\
0.320 E-02 \\
0.720 E-02 \\
0.000 E+00\end{array}$ \\
\hline $10 / 18 / 75$ & & $0.2930 \mathrm{E}+00$ & $0.000 \mathrm{E}+00$ \\
\hline
\end{tabular}

2 Miles 5 of 200 West ost 4 Depth 0-1 in.

$\begin{array}{lll}\text { Sample } & \begin{array}{l}\text { Dup. } \\ \text { Nate }\end{array} & \begin{array}{l}\text { Result } \\ \text { NCi }\end{array} \\ -05 / 01 / 86 & & 0.2300 E+00 \\ 05 / 31 / 85 & & 0.3720 E+00 \\ 05 / 17 / 84 & & 0.1390 E+00 \\ 08 / 05 / 83 & & 0.2770 E+00 \\ 08 / 24 / 82 & & 0.5760 E-01\end{array}$

200 Wegt Control Plot 60

\begin{tabular}{llll}
6020 & Depth $0-1$ in. & Sr-90 \\
Sample & Dup. & Result & $+/-2$ \\
Date & No. & pCi/g & std. Dev. \\
\hline $08 / 18 / 75$ & & $0.2250 E+00$ & $0.000 E+00$ \\
$09 / 24 / 74$ & & $0.3130 E+00$ & $0.000 E+00$ \\
$10 / 25 / 73$ & & $0.6580 E-01$ & $0.230 E-02$ \\
$10 / 25 / 72$ & & $0.3940 E+00$ & $0.000 E+00$ \\
$09 / 29 / 71$ & & $0.6520 E+00$ & $0.000 E+00$
\end{tabular}

200 West Control Plot 60

6043 Depth 1-2 in.

Sample Dup. Result $+/-2$

Date No.

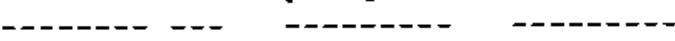

$10 / 25 / 73 \quad 0.1280 E+00 \quad 0.340 E-02$

$10 / 25 / 72 \quad 0.5050 E-01 \quad 0.000 E+00$

$09 / 30 / 71 \quad 0.1100 \mathrm{E}+00 \quad 0.000 \mathrm{E}+00$
200 West Control Plot 60

6191 Depth 2-4 in. Sr-90

Sample Dup. Result $+/-2$

Date No. pci/g std. Dev.

$\overline{10 / 25 / 73}=0.9460 \mathrm{E}-02 \quad \overline{0.230 \mathrm{E}-02}$

200 west Control Plot 60

6192 Depth 4-8 in. Sr-90

Sample Dup. Result $+/-2$

Date No. pCi/g std. Dev,

-------- -- - -

$10 / 25 / 73 \quad 0.4730 \mathrm{E}-02 \quad 0.230 \mathrm{E}-02$

200 West Control plot 60

6193 Depth $0-12$ in. sr-90

\begin{tabular}{llll} 
Sample & Dup. & Regult & $+/-2$ \\
Date & No. & PCi/g & Std. Dev. \\
\hdashline $10 / 25 / 73$ & & $\langle 0.1130 E-02$ & $0.250 E-02$
\end{tabular}

Other 200 Area Locations

200 Area Fire station

6021 Depth 0-1 in. Sr-90

Sample Dup. Result $+/-2$

Date No. pCi/g std. Dev.

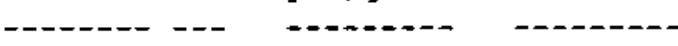

$10 / 25 / 73 \quad 0.3830 \mathrm{E}-01 \quad 0.230 \mathrm{E}-02$

$10 / 25 / 72 \quad 0.2220 \mathrm{E}+00 \quad 0.000 \mathrm{E}+00$

$09 / 30 / 71 \quad 0.2210 \mathrm{E}+00 \quad 0.000 \mathrm{E}+00$

200 Area Fire station

6044 Depth $1-2$ in. Sr-90

\begin{tabular}{llll} 
Sample & Dup. & Result & $+/-2$ \\
Date & No. & pCi/g & Std. Dev. \\
\hline $10 / 25 / 72$ & & $0.1080 \mathrm{E}+00$ & $0.000 \mathrm{E}+00$ \\
$09 / 30 / 71$ & & $0.3000 \mathrm{E}-01$ & $0.000 \mathrm{E}+00$
\end{tabular}


TABLE A.2. (contd)

other 200 Area tocations

\begin{tabular}{|c|c|c|c|}
\hline $\begin{array}{l}\text { Gable } \\
6321\end{array}$ & Depth & $0-1$ in. & Sr -90 \\
\hline $\begin{array}{l}\text { Sample } \\
\text { Date }\end{array}$ & $\begin{array}{l}\text { Dup. } \\
\text { No. }\end{array}$ & $\begin{array}{l}\text { Result } \\
\mathrm{pCi} / \mathrm{g}\end{array}$ & $\begin{array}{l}+/=2 \\
\text { std. Dev. }\end{array}$ \\
\hline $08 / 11 / 76$ & --- & $0.1020 \mathrm{E}+00$ & $0.000 \mathrm{E}+00$ \\
\hline $\begin{array}{l}\text { Inter. } \\
5223\end{array}$ & $\begin{array}{l}\text { S and } \\
\text { Depth }\end{array}$ & $\begin{array}{l}\text { Army Loop Rd. } \\
0-1 \text { in. }\end{array}$ & 'sr-90 \\
\hline $\begin{array}{l}\text { Sample } \\
\text { Date }\end{array}$ & $\begin{array}{l}\text { Dup. } \\
\text { No. }\end{array}$ & $\begin{array}{l}\text { Result } \\
\mathrm{pCi} / \mathrm{g}\end{array}$ & $\begin{array}{l}+/-2 \\
\text { std. Dev. }\end{array}$ \\
\hline $\begin{array}{l}O B / 10 / 76 \\
O B / 18 / 75 \\
09 / 24 / 74\end{array}$ & -- & $\begin{array}{l}0.4100 \mathrm{E}-01 \\
0.3250 \mathrm{E}+00 \\
0.8640 \mathrm{E}-01\end{array}$ & $\begin{array}{l}0.000 \mathrm{E}+00 \\
0.000 \mathrm{E}+00 \\
0.000 \mathrm{E}+00\end{array}$ \\
\hline
\end{tabular}

\begin{tabular}{|c|c|c|c|}
\hline $\begin{array}{l}\text { Army Loo } \\
6226\end{array}$ & $\begin{array}{l}\text { Rd. } \\
\text { Depth }\end{array}$ & $\begin{array}{l}\text { Contl. Plot } \\
0-1 \text { in. }\end{array}$ & $\begin{array}{l}40 \\
\text { Sr }-90\end{array}$ \\
\hline $\begin{array}{l}\text { Sample } \\
\text { Date }\end{array}$ & $\begin{array}{l}\text { Dup. } \\
\text { No. }\end{array}$ & $\begin{array}{l}\text { Result } \\
\mathrm{pCi} / \mathrm{g}\end{array}$ & $\begin{array}{l}+/{ }^{2} \\
\text { std. Dev. }\end{array}$ \\
\hline $09 / 24 / 74$ & --- & $0.1310 \mathrm{E}-01$ & $0.000 E+00$ \\
\hline
\end{tabular}

Highway 240 Control Plot 54

6002 Depth $0-1$ in. sr-90

\begin{tabular}{llll} 
Sample & Dup. & Result & $+/-2$ \\
Date & No. & pCi/g & Std. Dev. \\
\hline $09 / 23 / 74$ & & $0.4600 E-01$ & $0.000 E+00$ \\
$10 / 25 / 73$ & & $0.4260 E-01$ & $0.230 E-02$ \\
$10 / 25 / 72$ & & $0.2050 E+00$ & $0.000 E+00$ \\
$09 / 29 / 71$ & & $0.1610 \varepsilon+00$ & $0.000 \varepsilon+00$
\end{tabular}

Highway 240 Control plot 54

6025 Depth $1-2$ in. Sr-90

Sample Dup. Result $+/-2$

Date No. pCi/g std, Dev.

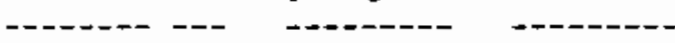

$10 / 25 / 72 \quad 0.93 \mathrm{BDE}-01 \quad 0.000 \mathrm{E}+00$

$09 / 29 / 71 \quad 0.3200 E-01 \quad 0.000 E+00$
300 Area

\begin{tabular}{llll}
$\begin{array}{l}\text { North of } \\
6322\end{array}$ & $\begin{array}{l}300 \text { Area } \\
\text { Depth } 0-1 \text { in. }\end{array}$ & st-90 \\
Sample & Dup. Regult & $+/-2$ \\
Date & No. & pCi/g & std. Dev. \\
\hline $04 / 29 / 87$ & & $0.2380 \mathrm{E}+00$ & $0.143 \mathrm{E}-01$ \\
$11 / 25 / 86$ & & $0.1840 \mathrm{E}+00$ & $0.181 \mathrm{E}-01$ \\
$05 / 31 / 85$ & $0.3230 \mathrm{E}+00$ & $0.232 \mathrm{E}-01$ \\
$05 / 16 / 84$ & $0.5810 \mathrm{E}+00$ & $0.287 \mathrm{E}-01$ \\
$08 / 11 / 83$ & $0.7330 \mathrm{E}+00$ & $0.225 \mathrm{E}-01$ \\
$09 / 03 / 82$ & $0.2220 \mathrm{E}+00$ & $0.343 \mathrm{E}-01$ \\
$08 / 10 / 76$ & & $0.7240 \mathrm{E}-01$ & $0.000 \mathrm{E}+00$
\end{tabular}

$\begin{aligned} & \text { North Center } 300 \text { Area Fence } \\ & 6279 \\ & \text { Depth } 0-1 \text { in. }\end{aligned}$
$\begin{array}{llll}\text { Sample } & \text { Dup. Regult } & +/-2 \\ \text { Date } & \text { No. } & \text { pCi/g } & \text { std. Dev. } \\ -08 / 20 / 75 & & 0.9300 E+00 & 0.000 E+00\end{array}$

300 Area South Gate

6222 Depth $0-1$ in. sr-90

\begin{tabular}{llll} 
Sample & Dup. & Result & $+/-2$ \\
Date & No. & PCi 2 & Std. Dev. \\
\hline $09 / 24 / 74$ & & $0.1230 E+00$ & $0.000 E+00$
\end{tabular}

\begin{tabular}{|c|c|c|c|}
\hline $\begin{array}{l}\text { South of } \\
6323\end{array}$ & $\begin{array}{l}300 \mathrm{Ar} \\
\text { Depth }\end{array}$ & $e^{a}$ in. & $S I-90$ \\
\hline $\begin{array}{l}\text { Sample } \\
\text { Date }\end{array}$ & $\begin{array}{l}\text { Dup. } \\
\text { No. }\end{array}$ & $\begin{array}{l}\text { Regult } \\
\mathrm{pCi} / \mathrm{g}\end{array}$ & $\begin{array}{l}+/-2 \\
\text { std. Dev. }\end{array}$ \\
\hline $\begin{array}{l}-0---1-- \\
04 / 29 / 86 \\
05 / 31 / 85 \\
05 / 16 / 84 \\
08 / 11 / 83 \\
09 / 03 / 82 \\
08 / 10 / 76\end{array}$ & --- & $\begin{array}{l}-\overline{0.3100 E+00} \\
0.5080 E+00 \\
0.3470 E+00 \\
0.1360 E+01 \\
0.2400 E+00 \\
0.5520 E-01\end{array}$ & $\begin{array}{l}0.110 \mathrm{E}-01 \\
0.248 \mathrm{E}-01 \\
0.150 \mathrm{E}-01 \\
0.386 \mathrm{E}-01 \\
0.145 \mathrm{E}+00 \\
0.000 \mathrm{E}+00\end{array}$ \\
\hline
\end{tabular}




\section{TABLE A.2. (contd)}

300 Area

331 Bldg. Control plot 50

$\begin{array}{llll}6014 & \text { Depth } 0-1 \text { in. } & \text { Sr-90 } \\ \text { Sample } & \text { Dup. } & \text { Result } & +/-2 \\ \text { Date } & \text { No. } & \text { pCi/g } & \text { std. Dev. } \\ -09 / 20 / 75 & & 0.4570 E-01 & 0.000 E+00 \\ 10 / 25 / 73 & & 0.1420 E-01 & 0.230 E-02 \\ 10 / 25 / 72 & & 0.5140 E-01 & 0.000 E+00 \\ 09 / 30 / 71 & & 0.2760 E+00 & 0.000 E+00\end{array}$

331 Bldg. Control Plot 50 6037 Depth 1-2 in.

Sr -90

\begin{tabular}{llll} 
Sample & Dup. & Result & $+/-2$ \\
Date & No. & pCi/g & Std. Dev. \\
\hline $10 / 25 / 72$ & & $0.5730 \mathrm{E}-01$ & $0.000 \mathrm{E}+00$ \\
$09 / 30 / 71$ & & $0.1960 \mathrm{E}+00$ & $0.000 \mathrm{E}+00$
\end{tabular}

400 srea and Vicinity

Wye Barricade

\begin{tabular}{|c|c|c|c|}
\hline $\begin{array}{l}\text { ample } \\
\text { ate }\end{array}$ & $\begin{array}{l}\text { Dup. } \\
\text { No. }\end{array}$ & $\begin{array}{l}\text { Result } \\
\mathrm{pCi} / \mathrm{g}\end{array}$ & $\begin{array}{l}+/=2 \\
\text { std. Dev. }\end{array}$ \\
\hline $\begin{array}{l}------- \\
04 / 29 / 87 \\
07 / 03 / 85 \\
05 / 15 / 84 \\
08 / 11 / 83 \\
08 / 16 / 82 \\
08 / 10 / 81 \\
08 / 05 / 80 \\
09 / 11 / 79 \\
08 / 08 / 78 \\
06 / 14 / 77 \\
10 / 25 / 73 \\
10 / 25 / 72 \\
09 / 30 / 71\end{array}$ & -- & $\begin{array}{l}-.-1- \\
0.1830 \mathrm{E}+00 \\
0.3070 \mathrm{E}+00 \\
0.3050 \mathrm{0}+00 \\
0.8100 \mathrm{E}+00 \\
0.2100 \mathrm{E}+00 \\
0.1980 \mathrm{E}+00 \\
0.2320 \mathrm{E}+00 \\
0.2600 \mathrm{E}+00 \\
0.3690 \mathrm{E}-01 \\
0.1400 \mathrm{E}+00 \\
0.1340 \mathrm{E}+00 \\
0.2850 \mathrm{E}+00 \\
0.2170 \mathrm{E}+00\end{array}$ & $\begin{array}{l}------- \\
0.878 \mathrm{E}-02 \\
0.186 \mathrm{E}-01 \\
0.442 \mathrm{E}-01 \\
0.258 \mathrm{E}-01 \\
0.265 \mathrm{E}-01 \\
0.230 \mathrm{E}-01 \\
0.680 \mathrm{E}-02 \\
0.870 \mathrm{E}-02 \\
0.370 \mathrm{E}-02 \\
0.810 \mathrm{E}-02 \\
0.360 \mathrm{E}-02 \\
0.000 \mathrm{E}+00 \\
0.000 \mathrm{E}+00\end{array}$ \\
\hline
\end{tabular}

Wye Barricade

\begin{tabular}{llll}
6039 & Depth & $1-2$ in. & sr-90 \\
& & & \\
Sample & Dup. & Regult & $+/-2$ \\
Date & No. & pCi/g & Std. Dev. \\
\hdashline $10 / 25 / 72$ & & $0.1750 \mathrm{E}+00$ & $0.000 \mathrm{E}+00$
\end{tabular}

\begin{tabular}{|c|c|c|c|}
\hline 0816 & Dept & $0-1 \mathrm{in}$. & $5 x-90$ \\
\hline $\begin{array}{l}\text { Sample } \\
\text { Date }\end{array}$ & $\begin{array}{l}\text { Dup. } \\
\text { No. }\end{array}$ & $\begin{array}{l}\text { Regule } \\
\text { pCi/g }\end{array}$ & $\begin{array}{l}+/-2 \\
\text { std. Dev. }\end{array}$ \\
\hline $\begin{array}{l}-1 / 29 / 87 \\
04 / 29 / 8 \\
04 / 29 / 86 \\
07 / 03 / 85 \\
06 / 06 / 84 \\
08 / 11 / 83 \\
08 / 16 / 82\end{array}$ & --- & $\begin{array}{l}0.1250 \mathrm{E}+00 \\
0.4510 \mathrm{E}-01 \\
0.2610 \mathrm{E}+00 \\
0.1580 \mathrm{E}+00 \\
0.9190 \mathrm{E}+00 \\
0.2400 \mathrm{E}+00\end{array}$ & $\begin{array}{l}0.826 \mathrm{E}-02 \\
0.453 \mathrm{E}-02 \\
0.219 \mathrm{E}-01 \\
0.119 \mathrm{E}-01 \\
0.252 \mathrm{E}-01 \\
0.511 \mathrm{E}-01\end{array}$ \\
\hline
\end{tabular}

\begin{tabular}{|c|c|c|c|}
\hline \multicolumn{3}{|c|}{ Wye Barricade (OC Dupl. B) } & $5 r-90$ \\
\hline $\begin{array}{l}\text { Sample } \\
\text { Date }\end{array}$ & $\begin{array}{l}\text { Dup. } \\
\text { No. }\end{array}$ & $\begin{array}{l}\text { Result } \\
\mathrm{pCi} / \mathrm{g}\end{array}$ & $\begin{array}{l}+/-2 \\
\text { std. Dev. }\end{array}$ \\
\hline $\begin{array}{l}-1-1 / 29 \\
04 / 29 / 87 \\
04 / 29 / 86 \\
07 / 03 / 85 \\
06 / 06 / 84 \\
08 / 11 / 83 \\
08 / 16 / 82\end{array}$ & --- & $\begin{array}{l}0.1580 \mathrm{E}+00 \\
0.4130 \mathrm{E}-01 \\
0.4410 \mathrm{E}+00 \\
0.1430 \mathrm{E}+00 \\
0.9350 \mathrm{E}+00 \\
0.2420 \mathrm{E}+00\end{array}$ & $\begin{array}{l}0.942 \mathrm{E}-02 \\
0.432 \mathrm{E}-02 \\
0.247 \mathrm{E}-01 \\
0.834 \mathrm{E}-02 \\
0.287 \mathrm{E}-01 \\
0.422 \mathrm{E}-01\end{array}$ \\
\hline
\end{tabular}

1 Mile $N$ of WPPSS No. 2

\begin{tabular}{|c|c|c|c|}
\hline 324 & Depth & $-1 i$ & sr-9o \\
\hline $\begin{array}{l}\text { Sample } \\
\text { Date }\end{array}$ & $\begin{array}{l}\text { Dup. } \\
\text { No. }\end{array}$ & $\begin{array}{l}\text { Regult } \\
\mathrm{pCi} / \mathrm{g}\end{array}$ & $\begin{array}{l}+/-2 \\
\text { std. Dev. }\end{array}$ \\
\hline $08 / 10 / 76$ & 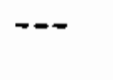 & $0.6940 \mathrm{E}-02$ & $0.000 \mathrm{E}+00$ \\
\hline $\begin{array}{l}1 \text { Hile } \mathrm{S} \\
6325\end{array}$ & $\begin{array}{l}\text { of WP } \\
\text { Depth }\end{array}$ & $\begin{array}{l}\text { PSS No. } 2 \\
\text { o-1 in. }\end{array}$ & $S I-90$ \\
\hline $\begin{array}{l}\text { Sample } \\
\text { Date }\end{array}$ & $\begin{array}{l}\text { Dup. } \\
\text { No. }\end{array}$ & $\begin{array}{l}\text { Result } \\
\mathrm{pCi} / \mathrm{g}\end{array}$ & $\begin{array}{l}+/-2 \\
\text { std. Dev. }\end{array}$ \\
\hline $08 / 10 / 76$ & $-\infty$ & $0.1520 \mathrm{E}+00$ & $0.000 \mathrm{E}+00$ \\
\hline
\end{tabular}

FFTF Control Plot 62

6015 Depth $0-1$ in. Sr-90

Sample Dup. Result $t /-2$

Date No. pCi/g std. Dev.

- -

$09 / 24 / 74 \quad 0.1780 \mathrm{E}-01 \quad 0.000 \mathrm{E}+00$

$10 / 25 / 73 \quad 0.75708-01 \quad 0.270 E-02$

$10 / 25 / 72 \quad 0.4530 \mathrm{E}-01 \quad 0.000 \mathrm{E}+00$

$09 / 30 / 71 \quad 0.9200 E-01 \quad 0.000 E+00$ 
TABLE A.2. (contd)

400 Arearand Vicinity

Other Onsite Locations

$\begin{array}{llll}\text { FFTF Control plot } 62 & \\ 6038 & \text { Depth } 1-2 \text { in. } & \text { Sr-90 } \\ & & & \\ \text { Sample } & \text { Dup. Regult } & +/-2 \\ \text { Date } & \text { No. } & \text { pCi/g } & \text { std. Dev. } \\ -10 / 25 / 72 & -- & 0.1920 \mathrm{E}-01 & 0.000 \mathrm{E}+00 \\ 09 / 30 / 71 & & 0.9100 \mathrm{E}-01 & 0.000 \mathrm{E}+00\end{array}$

0.5 Mile NE of FFTF

\begin{tabular}{|c|c|c|c|}
\hline $2 \theta 2$ & Depth & o-1 in. & SI -90 \\
\hline $\begin{array}{l}\text { Sample } \\
\text { Date }\end{array}$ & $\begin{array}{l}\text { Dup. } \\
\text { No. }\end{array}$ & $\begin{array}{l}\text { Regult } \\
\mathrm{pCi} / \mathrm{g}\end{array}$ & $\begin{array}{l}+/{ }^{2} \\
\text { std. Dev. }\end{array}$ \\
\hline $\begin{array}{l}04 / 29 / 87 \\
04 / 295 / 85 \\
06 / 05 / 85 \\
05 / 17 / 84 \\
08 / 12 / 83 \\
00 / 30 / 82 \\
08 / 10 / 81 \\
08 / 05 / 80 \\
09 / 11 / 79 \\
0 \theta / 00 / 78 \\
06 / 14 / 77 \\
08 / 10 / 76 \\
08 / 20 / 75\end{array}$ & --- & $\begin{array}{l}0.8550 \mathrm{E}-01 \\
0.1720 \mathrm{E}+00 \\
0.1780 \mathrm{E}+00 \\
0.5190 \mathrm{E}+00 \\
0.4150 \mathrm{E}-01 \\
0.3780 \mathrm{E}-01 \\
0.4160 \mathrm{E}-01 \\
0.8530 \mathrm{E}-01 \\
0.4260 \mathrm{E}-01 \\
0.1850 \mathrm{E}-01 \\
0.3160 \mathrm{E}+00 \\
0.5940 \mathrm{E}-01\end{array}$ & $\begin{array}{l}0.050 \mathrm{E}-02 \\
0.179 \mathrm{E}-01 \\
0.208 \mathrm{E}-01 \\
0.204 \mathrm{E}-01 \\
0.350 \mathrm{E}-01 \\
0.740 \mathrm{E}-02 \\
0.110 \mathrm{E}-01 \\
0.670 \mathrm{E}-02 \\
0.460 \mathrm{E}-02 \\
0.360 \mathrm{E}-02 \\
0.000 \mathrm{E}+00 \\
0.000 \mathrm{E}+00\end{array}$ \\
\hline
\end{tabular}

$N$ End of Vernita Bridge

6005 Depth $0-1$ in.

\begin{tabular}{llll} 
Sample & Dup. & Result & $+/-2$ \\
Date & No. & pCi/g & Std. Dev. \\
\hline $05 / 02 / 86$ & & $0.6800 \mathrm{E}-01$ & $0.608 \mathrm{E}-02$ \\
$06 / 11 / 85$ & & $0.3090 \mathrm{E}+00$ & $0.209 \mathrm{E}-01$ \\
$05 / 31 / 84$ & & $0.1670 \mathrm{E}+00$ & $0.149 \mathrm{~A}-01$ \\
$00 / 11 / 83$ & & $0.5190 \mathrm{E}+00$ & $0.172 \mathrm{E}-01$ \\
$08 / 24 / 82$ & & $0.1080 \mathrm{E}+00$ & $0.655 \mathrm{E}-01$ \\
$09 / 23 / 74$ & $0.2520 \mathrm{E}-01$ & $0.000 \mathrm{E}+00$ \\
$10 / 25 / 73$ & & $0.1270 \mathrm{E}+00$ & $0.320 \mathrm{E}-02$ \\
$10 / 25 / 72$ & $0.9650 \mathrm{E}-01$ & $0.000 \mathrm{D}+00$ \\
$09 / 29 / 71$ & & $0.8000 \mathrm{E}-01$ & $0.000 \mathrm{E}+00$
\end{tabular}

N End of Vernita Bridge

$602 \theta$ Depth 1-2 in.

Sample Dup. Result $t /-^{2}$

Date No. $\mathrm{pCi} / \mathrm{g}$ std. Dev.

$\overline{10 / 25 / 72}-1 \quad 0.4330 \mathrm{E}-01000$

$09 / 29 / 71 \quad 0.8000 E-01 \quad 0.000 E+00$

Hanford Townaite

\begin{tabular}{|c|c|c|c|}
\hline 6017 & Depth & $0-1 \mathrm{in}$. & $\$ r-90$ \\
\hline $\begin{array}{l}\text { Sample } \\
\text { Date }\end{array}$ & $\begin{array}{l}\text { Dup. } \\
\text { No. }\end{array}$ & $\begin{array}{l}\text { Result } \\
\text { pCi/g }\end{array}$ & $\begin{array}{l}+/-2 \\
\text { std. Dev. }\end{array}$ \\
\hline ד & --- & $---1-2$ & (2) \\
\hline $04 / 29 / 87$ & & $0.2 \theta 005+00$ & $0.118 \mathrm{E}-01$ \\
\hline 85 & & $E+00$ & 0 \\
\hline $05 / 17 / 84$ & & $0.3110 \mathrm{E}+00$ & $0.293 \mathrm{E}-01$ \\
\hline $2 / 83$ & & $0.1920 \mathrm{E}+01$ & $0.478 \mathrm{E}-01$ \\
\hline$/ 82$ & & $0.2420 \mathrm{E}+00$ & $0.797 \mathrm{E}-01$ \\
\hline $0 / 81$ & & $0.3990 \mathrm{E}+00$ & $0.430 \mathrm{E}-02$ \\
\hline $08 /$ & & 0.2280 & $0.700 \mathrm{E}-02$ \\
\hline $09 / 11 / 79$ & & $0.2040 \mathrm{E}+00$ & $0.860 \mathrm{E}-02$ \\
\hline $08 / 08 / 78$ & & $0.3120 \mathrm{E}-01$ & $0.370 \mathrm{E}-02$ \\
\hline$\$ 77$ & & $0.3400 \mathrm{E}-01$ & $0.360 \mathrm{E}-02$ \\
\hline $08 / 11 / 76$ & & $0.1230 \mathrm{E}+00$ & $0.000 \mathrm{E}+00$ \\
\hline $08 / 18 / 75$ & & $0.4050 \mathrm{E}+00$ & $0.000 \mathrm{E}+00$ \\
\hline $10 / 2$ & & $0.3320 \mathrm{E}+00$ & $0.520 \mathrm{E}-02$ \\
\hline $10 / 25 / 72$ & & $0.3340 E+00$ & $0.0008+00$ \\
\hline $09 / 30 / 71$ & & $0.67208+00$ & $0.000 \mathrm{E}+00$ \\
\hline
\end{tabular}


TABLE A.2. (contd)

Other onsite Lecationg

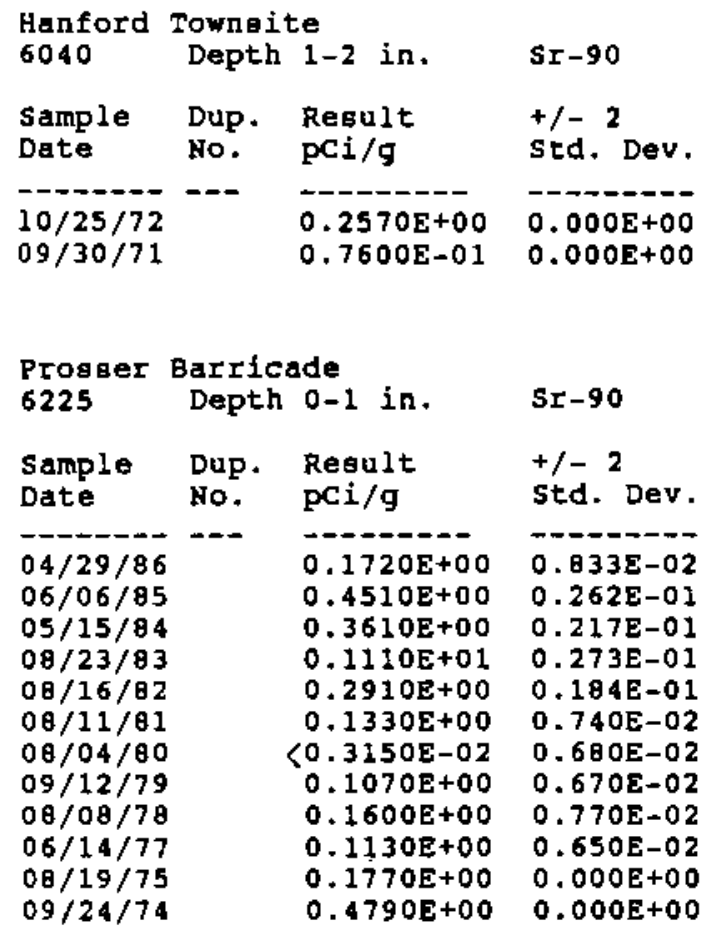

$\begin{array}{llll}\text { ERC (Emer. Reloc. Center) } & \\ 6001 & \text { Depth 0-1 in. } & \text { Sr-90 } \\ \text { Sample } & \text { Dup. Result } & +/-2 \\ \text { Date } & \text { No. } & \text { pCi/g } & \text { Std. Dev. } \\ -09 / 24 / 74 & & 0.1750 E-01 & 0.000 \mathrm{E}+00 \\ 10 / 25 / 73 & & 0.7570 \mathrm{E}-01 & 0.270 \mathrm{E}-02 \\ 10 / 25 / 72 & 0.9650 \mathrm{E}-01 & 0.000 \mathrm{E}+00 \\ 09 / 30 / 71 & & 0.1490 \mathrm{E}+00 & 0.000 \mathrm{E}+00\end{array}$

ERC (Emer. Reloc. Center)

\begin{tabular}{llll}
6024 & Depth $1-2$ in. & Sr-90 \\
Sample & Dup. & Result & $+/-2$ \\
Date & No. & pCi $/ g$ & std. Dev. \\
\hline $10 / 25 / 72$ & & $0.3440 E-01$ & $0.000 E+00$ \\
$09 / 30 / 71$ & & $0.1020 \mathrm{E}+00$ & $0.000 \mathrm{E}+00$
\end{tabular}

$E$ of Arid Land Ecol. Lab

6278 Depth $0-1$ in.

$\operatorname{sr}-90$

Sample Dup. Regult

Date No. pCi/g

$05 / 20 / 87$

$06 / 12 / 85$

$05 / 24 / 84$

$08 / 23 / 83$

$08 / 16 / 82$

$08 / 13 / \theta 1$

$08 / 05 / 80$

$09 / 12 / 79$

$08 / 10 / 78$

$06 / 14 / 77$

$08 / 10 / 76$

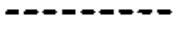

$0.3980 E+00$

$0.6070 \mathrm{E}+00$

$0.3580 \mathrm{E}+00$

$0.1630 \mathrm{E}+01$

$0.3000 \mathrm{E}+00$

$0.2720 \mathrm{E}+00$

$<0.0000 E+00$

$0.3700 \mathrm{E}+00$

$0.5770 \mathrm{E}-02$

$0.7570 \mathrm{E}-01$

$0.1540 E-01$

$0.4690 \mathrm{E}+00$

$+/-2$

std. Dev.

$0.256 \mathrm{E}-01$

$0.349 \mathrm{E}-01$

$0.394 \mathrm{E}-01$

$0.323 E-01$

$0.504 \mathrm{E}-01$

$0.340 E-01$

$0.600 E-02$

$0.100 E-01$

$0.330 E-02$

$0.560 E-02$

$0.000 E+00$

$0.000 E+00$

Rattlesnake Springo

\begin{tabular}{llll}
6003 & Depth & $0-1$ in. & $5 r-90$ \\
Sample & Dup. & Regult & $+/-2$ \\
Date & No. & pCi/g & std. Dev. \\
\hline $05 / 07 / 87$ & & $0.1230 \mathrm{E}+00$ & $0.134 \mathrm{E}-01$ \\
$05 / 23 / 86$ & & $0.1800 \mathrm{E}+00$ & $0.801 \mathrm{E}-02$ \\
$06 / 06 / 85$ & & $0.3280 \mathrm{E}+00$ & $0.243 \mathrm{E}-01$ \\
$05 / 24 / 84$ & & $0.7450 \mathrm{E}-01$ & $0.883 \mathrm{E}-02$ \\
$00 / 11 / 83$ & & $0.0880 \mathrm{E}+00$ & $0.331 \mathrm{E}-01$ \\
$09 / 02 / 82$ & & $0.1710 \mathrm{E}+00$ & $0.381 \mathrm{E}-01$ \\
$09 / 23 / 74$ & & $0.3440 \mathrm{E}+00$ & $0.000 \mathrm{E}+00$ \\
$10 / 25 / 73$ & & $0.7300 \mathrm{E}-01$ & $0.270 \mathrm{E}-02$ \\
$10 / 25 / 72$ & & $0.1380 \mathrm{E}-01$ & $0.000 \mathrm{E}+00$
\end{tabular}

Rattlesnake Springs

\begin{tabular}{llll}
6026 & Depth $1-2$ in. & Sr-90 \\
Sample & Dup. & Result & $+/-2$ \\
Date & No. & pCi/g & Std. Dev. \\
\hline $10 / 25 / 72$ & & $0.3060 E-01$ & $0.000 E+00$ \\
$09 / 29 / 71$ & & $0.4400 E-01$ & $0.000 E+00$
\end{tabular}


TABLE A.2. (contd)

other Onsite Locations

Yakima Barricade

\begin{tabular}{|c|c|c|c|}
\hline 004 & Dept & $0-1$ in. & $5 r-90$ \\
\hline $\begin{array}{l}\text { Sample } \\
\text { Date }\end{array}$ & $\begin{array}{l}\text { Dup. } \\
\text { No. }\end{array}$ & $\begin{array}{l}\text { Result } \\
\mathrm{pCi} / \mathrm{g}\end{array}$ & $\begin{array}{l}+/-2 \\
\text { std. Dev. }\end{array}$ \\
\hline 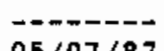 & 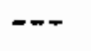 & יаים & -- \\
\hline $\begin{array}{l}05 / 07 / 87 \\
06 / 06 / 85\end{array}$ & & $\begin{array}{l}0.6390 \mathrm{E}-01 \\
0.5370 \mathrm{E}+00\end{array}$ & $\begin{array}{l}0.121 E-01 \\
0.293 E-01\end{array}$ \\
\hline $05 / 24 / 04$ & & $0.1340 \mathrm{E}+00$ & $0.173 E-01$ \\
\hline $08 / 11 / 83$ & & $0.5910 E+00$ & $0.226 \mathrm{E}-01$ \\
\hline $08 / 17 / 82$ & & $0.9040 E-01$ & $0.337 \mathrm{E}-02$ \\
\hline $08 / 11 / 81$ & & $0.2230 E+00$ & $\begin{array}{l}0.230 \mathrm{E}-01 \\
0.700 \mathrm{E}-02\end{array}$ \\
\hline $\begin{array}{l}0 \theta / 04 / 80 \\
09 / 11 / 79\end{array}$ & & $0.1600 \mathrm{E}-01$ & $\begin{array}{l}0.700 E-02 \\
0.120 E-01\end{array}$ \\
\hline $\begin{array}{l}09 / 11 / 79 \\
08 / 08 / 78\end{array}$ & & $0.9490 \mathrm{E}-02$ & $0.300 \mathrm{E}-02$ \\
\hline $06 / 14 / 77$ & & $0.1300 \mathrm{E}-01$ & $0.340 E-02$ \\
\hline $08 / 10 / 76$ & & $0.1520 \mathrm{E}+00$ & $0.000 E+00$ \\
\hline & & $0.1210 \mathrm{E}+00$ & $0.000 E+00$ \\
\hline $\begin{array}{l}09 / 23 / 74 \\
10 / 25 / 73\end{array}$ & & $0.1310 E-01$ & $\begin{array}{l}0.000 E+00 \\
0.00 E-02\end{array}$ \\
\hline $5 / 72$ & & $E+00$ & $0.000 \mathrm{E}+00$ \\
\hline & & $0 \mathrm{E}+00$ & $0.000 E+00$ \\
\hline
\end{tabular}

Yakima Barricade

6027 Depth 1-2 in. Sr-90

Sample Dup, Result $t /-2$

Date No. pCi/g std. Dev.

$\overline{10 / 25 / 72}=0 . \overline{0.1070 \mathrm{E}+00} 0 . \overline{00 \mathrm{E}+00}$

$09 / 29 / 71 \quad 0.1590 E+00 \quad 0.000 E+00$ 
TABLE A.3. Cesium-137 Concentrations in Soil at Onsite Locations

100 Areas

NE Corner of $100 \mathrm{~N}$ Area

6275 Depth $0-1$ in. Cs-137

\begin{tabular}{llll} 
Sample & Dup. Result & $+/ \overrightarrow{2}^{2}$ \\
Date & No. & PCi $/ g$ & std. Dev. \\
\hdashline $08 / 20 / 75$ & & $0.1180 \mathrm{E}+01$ & $0.000 \mathrm{E}+00$
\end{tabular}

1 Mile NE of $100 \mathrm{~N}$ Area

0590 Depth 0-1 in.

\begin{tabular}{llll} 
Sample & Dup. & Regult & $+/-2$ \\
Date & No. & PCi/g & Std. Dev. \\
\hline $04 / 30 / 86$ & & $0.8120 E+00$ & $0.484 E-01$ \\
$06 / 24 / 85$ & & $0.7620 E+00$ & $0.457 E-01$ \\
$05 / 17 / 84$ & & $0.7040 E+00$ & $0.482 E-01$ \\
$08 / 05 / 83$ & $0.4790 E+00$ & $0.403 E-01$ \\
$08 / 24 / 82$ & & $0.6690 \mathrm{E}+00$ & $0.812 E-01$
\end{tabular}

1 Mile E of $100 \mathrm{~N}$ Area 0578 Depth $0-1$ in.

\begin{tabular}{llll} 
Sample & Dup. & Regult & $+/-2$ \\
Date & No. & pCi/g & std. Dev. \\
\hline $04 / 29 / 87$ & & $0.1080 E+01$ & $0.521 E-01$ \\
$04 / 30 / 86$ & & $0.5540 E+00$ & $0.392 E-01$ \\
$06 / 24 / 85$ & & $0.6200 E+00$ & $0.437 E-01$ \\
$05 / 17 / 84$ & & $0.6660 E+00$ & $0.445 E-01$ \\
$08 / 11 / 83$ & & $0.7670 E+00$ & $0.515 E-01$ \\
$08 / 24 / 82$ & & $0.5390 E+00$ & $0.391 E-01$
\end{tabular}

1 Mile SE of $100 \mathrm{~N}$ Trench

$\begin{array}{llll}6320 & \text { Depth } 0-1 \text { in. } & \text { C6-137 } \\ & & & \\ \text { Sample } & \text { Dup. } & \text { Result } & +/-2 \\ \text { Date } & \text { No. } & \text { pCi/g } & \text { Std. Dev. } \\ -3 / 03 / 84 & & 0.8180 \mathrm{E}+00 & 0.487 \mathrm{E}-01 \\ 08 / 11 / 76 & & 0.4180 \mathrm{E}+00 & 0.000 \mathrm{E}+00\end{array}$

Wahluke slope Opposite 100-N

6006 Depth 0-1 in. Cs-137

\begin{tabular}{llll} 
Sample & Dup. & Result & $+/-^{2}$ \\
Date & No. & pCi/g & std. Dev. \\
\hline $09 / 23 / 74$ & & $0.1470 E+00$ & $0.000 E+00$ \\
$10 / 25 / 73$ & & $0.1200 E+01$ & $0.000 E+00$ \\
$10 / 25 / 72$ & & $0.6520 E+00$ & $0.000 E+00$ \\
$09 / 29 / 71$ & & $0.8160 E+00$ & $0.000 E+00$
\end{tabular}

wahluke slope Opposite 100-N

6029 Depth 1-2 in. Cs-137

\begin{tabular}{llll} 
Sample & Dup. & Result & $+/-2$ \\
Date & No. & pCi/g & std. Dev. \\
\hline $10 / 25 / 72$ & & $0.4790 \mathrm{E}+00$ & $0.000 \mathrm{E}+00$ \\
$09 / 29 / 71$ & & $0.7470 \mathrm{E}-01$ & $0.000 \mathrm{E}+00$
\end{tabular}

100 Aree Fire station

0580 Depth 0-1 in. Cs-137

Sample Dup. Result $+/-2$

Date No. pCi/g std. Dev.

- - - - -

$04 / 30 / 86 \quad 0.1580 E+01 \quad 0.679 \mathrm{E}-01$

$05 / 30 / 85 \quad 0.1160 E+01 \quad 0.430 E-0 I$

$05 / 17 / 04 \quad 0.98408+00 \quad 0.569 E-01$

$08 / 05 / 83 \quad 0.1410 E+01 \quad 0.662 E-01$

$08 / 24 / 82 \quad 0.9850 E+00 \quad 0.603 E-01$

100 F Area Control plot 58

6018 Depth $0-1$ in. Ca-137

\begin{tabular}{llll} 
Sample & Dup. & Regult & $+/-2$ \\
Date & No. & pCi/g & Std. Dev. \\
\hline $08 / 11 / 76$ & & $0.4790 \mathrm{E}+00$ & $0.000 \mathrm{E}+00$ \\
$08 / 18 / 75$ & & $0.9200 \mathrm{E}+00$ & $0.000 \mathrm{E}+00$ \\
$09 / 24 / 74$ & & $0.6110 \mathrm{E}+00$ & $0.000 \mathrm{E}+00$ \\
$10 / 25 / 73$ & & $0.7330 \mathrm{E}+00$ & $0.000 \mathrm{E}+00$ \\
$10 / 25 / 77$ & & $0.6500 \mathrm{E}+00$ & $0.000 \mathrm{E}+00$ \\
$09 / 30 / 71$. & & $0.5120 \mathrm{E}+00$ & $0.000 \mathrm{E}+00$
\end{tabular}

$\begin{array}{llll}100 \text { F Area Control Plot 58 } & \\ 6041 & \text { Depth } 1-2 \text { in. } & \text { Cs }-137 \\ & & & \\ \text { Sample } & \text { Dup. Regult } & +/-2 \\ \text { Date } & \text { No. } & \text { pCi/g } & \text { Std. Dev. } \\ & & & \\ 10 / 25 / 72 & & 0.4070 \mathrm{E}+00 & 0.000 \mathrm{E}+00 \\ 09 / 30 / 71 . & <0.0000 \mathrm{E}+00 & 0.000 \mathrm{E}+00\end{array}$


TABLE A.3. (contd)

200 East Area

\begin{tabular}{llll}
200 E Control Plot 59 & \\
6019 & Depth $0-1$ in. & Cg-137 \\
Sample & Dup. & Regult & $+/-2$ \\
Date & No. & pC1/g & Std. Dev. \\
\hline $08 / 18 / 75$ & & $0.2460 E+01$ & $0.000 E+00$ \\
$09 / 24 / 74$ & $0.2910 E+01$ & $0.000 E+00$ \\
$10 / 25 / 73$ & $0.3430 E+00$ & $0.000 E+00$ \\
$10 / 25 / 72$ & $0.4880 E+00$ & $0.000 E+00$ \\
$09 / 30 / 71$ & $0.1290 E+00$ & $0.000 E+00$
\end{tabular}

\begin{tabular}{|c|c|c|c|}
\hline $\begin{array}{l}200 \mathrm{E} \\
6042\end{array}$ & Depth & in. & $\mathrm{Cs}-237$ \\
\hline $\begin{array}{l}\text { Tample } \\
\text { jate }\end{array}$ & $\begin{array}{l}\text { Dup. } \\
\text { No. }\end{array}$ & $\begin{array}{l}\text { Regult } \\
\mathrm{pCi} / \mathrm{g}\end{array}$ & $\begin{array}{l}+/-2 \\
\text { std. Dev. }\end{array}$ \\
\hline $\begin{array}{l}10 / 25 / 73 \\
10 / 25 / 72 \\
09 / 29 / 71\end{array}$ & -- & $\begin{array}{l}0.1700 \mathrm{E}+00 \\
0.2900 \mathrm{E}+00 \\
0.5400 \mathrm{E}-01\end{array}$ & $\begin{array}{l}0.000 E+00 \\
0.000 E+00 \\
0.000 E+00\end{array}$ \\
\hline
\end{tabular}

200 E control Plat 59

6180 Depth 2-4 in. Cs-137

\begin{tabular}{llll} 
Sample & Dup. & Result & $+/ 2$ \\
Date & No. & pC1/g & std. Dev. \\
\hdashline $10 / 25 / 73$ & & $0.3600 \mathrm{D}-01$ & $0.000 \mathrm{E}+00$
\end{tabular}

\begin{tabular}{llll}
200 E Control Plot 59 & \\
6189 & Depth $4-8$ in. & Cs-137 \\
& & & \\
Sample & Dup. Result & $+/-2$ \\
Date & No. pCi/g & std. Dev. \\
\hdashline $10 / 25 / 73$ & & $0.1040 E+00$ & $0.000 E+00$
\end{tabular}

200 E Control Plot 59

\begin{tabular}{llll}
6190 & Depth $\theta-12$ in. & Cs-137 \\
Sample & Dup. & Result & $+/-2$ \\
Date & No. & pCi/g & std. Dev. \\
\hline $10 / 25 / 73$ & & $0.2300 \mathrm{E}-01$ & $0.000 \mathrm{E}+00$
\end{tabular}

200 ENC (East North Central)

6362 Depth $0-1$ in. Cs-137

Sample Dup. Result $+/-2$

Date No. pCi/g std. Dev.

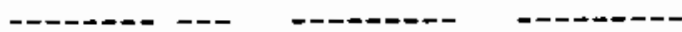

$05 / 07 / 87 \quad 0.1600 \varepsilon+02 \quad 0.191 E+00$

$04 / 30 / 86 \quad 0.9570 \mathrm{E}+01 \quad 0.146 \mathrm{E}+00$

$05 / 31 / 85 \quad 0.2280 \mathrm{E}+02 \quad 0.229 \mathrm{E}+00$

$05 / 17 / 84 \quad 0.2120 E+02 \quad 0.233 E+00$

$0 \theta / 12 / \theta 3 \quad 0.2 \theta 10 E+02 \quad 0.32 \theta E+00$

$08 / 30 / 82 \quad 0.2240 E+02,0.373 E+00$

$08 / 10 / 811 \quad 0.1810 \mathrm{E}+02 \quad 0.160 \mathrm{E}+00$

$08 / 10 / 81 \quad 0.1240 E+02 \quad 0.160 E+00$

$08 / 05 / 80 \quad 0.2750 E+02 \quad 0.440 E+00$

$09 / 11 / 79 \quad 0.3520 E+02 \quad 0.500 E+00$

$0 \theta / 10 / 78 \quad 0.1640 \mathrm{E}+01 \quad 0.120 \mathrm{E}+00$

$06 / 14 / 77 \quad 0.2860 E+02 \quad 0.460 E+00$

1.25 Mileg East of PUREX

0581 Depth 0-1 in. Cs-137

\begin{tabular}{|c|c|c|c|}
\hline $\begin{array}{l}\text { Sample } \\
\text { jate }\end{array}$ & $\begin{array}{l}\text { Dup. } \\
\text { No. }\end{array}$ & $\begin{array}{l}\text { Regult } \\
\text { pci/g }\end{array}$ & $\begin{array}{l}+/-2 \\
\text { std. Dev. }\end{array}$ \\
\hline $\begin{array}{l}----1- \\
05 / 07 / 87 \\
04 / 30 / 86 \\
05 / 31 / 85 \\
05 / 15 / 84 \\
08 / 05 / 83 \\
08 / 24 / 82\end{array}$ & --- & $\begin{array}{l}-.6890 \mathrm{E}+00 \\
0.600 \\
0.1400 \mathrm{E}+01 \\
0.3010 \mathrm{E}+01 \\
0.1410 \mathrm{E}+01 \\
0.1290 \mathrm{E}+01 \\
0.1450 \mathrm{E}+01\end{array}$ & $\begin{array}{l}0.364 \mathrm{E}-01 \\
0.543 \mathrm{E}-01 \\
0.900 \mathrm{E}-01 \\
0.573 \mathrm{E}-01 \\
0.669 \mathrm{E}-01 \\
0.978 \mathrm{E}-01\end{array}$ \\
\hline
\end{tabular}


TABLE A. 3. (contd)

200 East Area

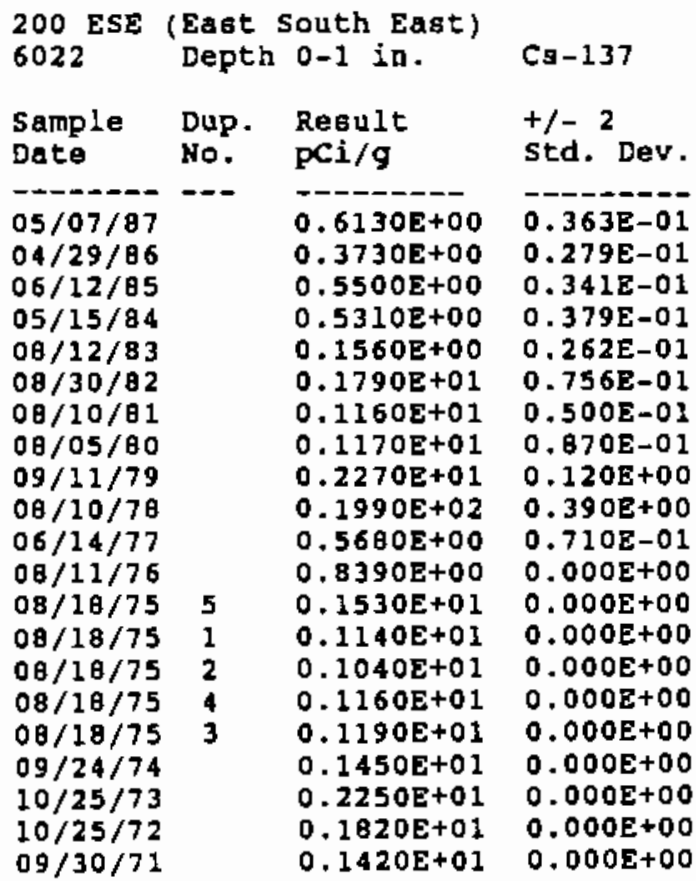

200 ESE (Eagt South Eagt)

$\mathrm{Cg}-137$

200 ESE (East South Eart)

6195 Depth 4-8 in.

$\mathrm{Cs}-137$

Sample Dup. Result $\quad+/-2$

Date No. $\mathrm{pCi} / \mathrm{g}$ std. Dev.

$\overline{10 / 25 / 73}=0 . \overline{0.100 \mathrm{E}+00} \quad \overline{0.000 \mathrm{E}+00}$

200 ESE (East South East)

6196 Depth o-12 in. Cs-137

Sample Dup. Result $+/=2$

Date No. pCi/g std. Dev.

10/25/73 -

$10 / 25 / 73 \quad 0.1150 \mathrm{E}-01 \quad 0.000 \mathrm{E}+00$

2 Miles south of PUREX

0582 Depth 0-1 in.

$\mathrm{Cs}-137$

Sarple Dup. Result $+/-2$

Date No. $\mathrm{pCi} / \mathrm{g}$ std. Dev.

- - - - - - - - - - -

$05 / 07 / 87 \quad 0.1340 E+00 \quad 0.248 E-01$

$04 / 30 / 86 \quad 0.7110 \mathrm{E}+00 \quad 0.413 \mathrm{E}-01$

$06 / 24 / 85 \quad 0.5620 E+00 \quad 0.394 E-01$

$05 / 15 / 84 \quad 0.1390 E+00-0.203 E-01$

$08 / 05 / 83 \quad 0.1500 E+00 \quad 0.255 E-01$

$08 / 24 / 82 \quad 0.5400 \mathrm{E}+00 \quad 0.721 \mathrm{E}-01$

$\begin{array}{llll}\text { Sample } & \text { Dup. } & \text { Result } & +/-2 \\ \text { Date } & \text { No. } & \text { pCi/g } & \text { Std. Dev. } \\ -10 / 25 / 73 & & 0.4600 \mathrm{E}+00 & 0.000 \mathrm{E}+00 \\ 10 / 25 / 72 & & 0.1410 \mathrm{E}+00 & 0.000 \mathrm{0}+00 \\ 09 / 30 / 71 & & 0.1070 \mathrm{E}+00 & 0.000 \mathrm{E}+00\end{array}$

200 ESE (East South East)

6194 Depth 2-4 in. Cs-137

Sample Dup. Regult $+/-2$

Date No. pci/g std. Dev.

Std. Dev.

\begin{tabular}{|c|c|c|c|}
\hline $\begin{array}{l}\text { Hiles } \\
503\end{array}$ & $\begin{array}{l}\text { S5W of } \\
\text { Depth }\end{array}$ & $\begin{array}{l}\text { PUREX } \\
0-1 \text { in. }\end{array}$ & $C s-137$ \\
\hline $\begin{array}{l}\text { ample } \\
\text { ate }\end{array}$ & $\begin{array}{l}\text { Dup. } \\
\text { No. }\end{array}$ & $\begin{array}{l}\text { Regult } \\
\mathrm{pCi} / \mathrm{g}\end{array}$ & $\begin{array}{l}+/-2 \\
\text { std. Dev. }\end{array}$ \\
\hline - - - - - - & --- & 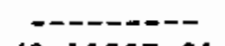 & 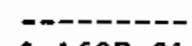 \\
\hline $\begin{array}{l}05 / 07 / 87 \\
04 / 30 / 86 \\
06 / 24 / 85 \\
05 / 15 / 84 \\
08 / 05 / 83\end{array}$ & & $\begin{array}{r}<0.1130 E-01 \\
0.1170 E+00 \\
0.1370 E+00 \\
0.6390 E-01 \\
0.3060 E+00 \\
0.3130 E+00\end{array}$ & $\begin{array}{l}0.160 E-01 \\
0.218 E-01 \\
0.208 E-01 \\
0.216 E-01 \\
0.369 E-01 \\
0.625 E-01\end{array}$ \\
\hline
\end{tabular}

$10 / 25 / 73 \quad 0.9270 E-01 \quad 0.000 E+00$

\begin{tabular}{llll}
$\begin{array}{l}\text { E Side of US Ecology } \\
6281\end{array}$ & Depth $0-1$ in. & Cs-137 \\
& & & \\
Sample & Dup. & Result & $+/-2$ \\
Date & No. & pCi/g & Std. Dev. \\
\hline $08 / 03 / 84$ & & $0.2080 E-01$ & $0.184 E-01$ \\
$08 / 11 / 76$ & & $0.6600 E+00$ & $0.000 E+00$ \\
$08 / 18 / 75$ & & $0.2160 E+00$ & $0.000 E+00$
\end{tabular}


TABLE A. 3. (contd)

200 West_Area

\begin{tabular}{llll} 
E of 200 & West Gate & \\
6276 & Depth $0-1$ in. & Cs-137 \\
Sample & Dup. & Result & $+/-2$ \\
Date & No. & pCi/g & std. Dev. \\
\hline $05 / 07 / 87$ & & $0.1300 E+01$ & $0.536 E-01$ \\
$05 / 01 / 86$ & $0.3080 E+01$ & $0.907 E-01$ \\
$05 / 31 / 85$ & $0.1970 E+01$ & $0.693 E-01$ \\
$05 / 15 / 84$ & $0.5850 E+00$ & $0.413 E-01$ \\
$08 / 12 / 83$ & $0.5660 E+01$ & $0.152 E+00$ \\
$08 / 30 / 82$ & $0.3210 E+01$ & $0.101 E+00$ \\
$08 / 10 / 81$ & $0.2530 E+01$ & $0.750 E-01$ \\
$08 / 04 / 80$ & $0.4780 E+01$ & $0.180 E+00$ \\
$09 / 11 / 79$ & $0.3840 E+01$ & $0.170 E+00$ \\
$08 / 09 / 78$ & $0.4200 E+01$ & $0.180 E+00$ \\
$06 / 14 / 77$ & $0.9710 E+00$ & $0.900 E-01$ \\
$08 / 11 / 76$ & $0.9070 E+00$ & $0.000 E+00$ \\
$08 / 18 / 75$ & $0.3010 E+01$ & $0.000 E+00$
\end{tabular}

\begin{tabular}{llll}
2 Milea & s of 200 Weat \\
0584 & Depth 0-1 in. & Cs-137 \\
Sample & Dup. Result & $+/-2$ \\
Date & No. & pC1/g & Std. Dev. \\
\hline $05 / 01 / 86$ & & $0.4950 E+00$ & $0.388 E-01$ \\
$05 / 31 / 85$ & & $0.3020 E+00$ & $0.303 E-01$ \\
$05 / 17 / 84$ & & $0.1670 E+00$ & $0.286 E-01$ \\
$08 / 05 / 83$ & & $0.6900 E-01$ & $0.216 E-01$ \\
$08 / 24 / 82$ & & $0.2110 E+00$ & $0.6228-01$
\end{tabular}

200 Weat Control Plot 60 6020 Depth 0-1 in.

\begin{tabular}{lll} 
Sample & Dup. & Regult \\
Date & No. & pCi/g \\
\hline $00 / 1 \theta / 75$ & & $0.3500 E+00$ \\
$09 / 24 / 74$ & $0.5340 E+00$ \\
$10 / 25 / 73$ & $0.2360 E+01$ \\
$10 / 25 / 72$ & $0.1440 E+01$ \\
$09 / 29 / 71$ & $0.1640 E+01$
\end{tabular}

200 west Control Plot 60

$\begin{array}{llll}6043 & \text { Depth } 1-2 \mathrm{in} . & \mathrm{Cg}-137 \\ \text { Sample } & \text { Dup. } & \text { Result } & +/{ }^{2} \\ \text { Date } & \text { No. } & \mathrm{pCi} / \mathrm{g} & \text { Std. Dev. } \\ -10 / 25 / 73 & & 0.4410 \mathrm{E}+00 & 0.00 \mathrm{OE}+00 \\ 10 / 25 / 72 & & 0.3080 \mathrm{E}+00 & 0.000 \mathrm{E}+00 \\ 09 / 30 / 71 & & 0.1190 \mathrm{E}+00 & 0.000 \mathrm{E}+00\end{array}$

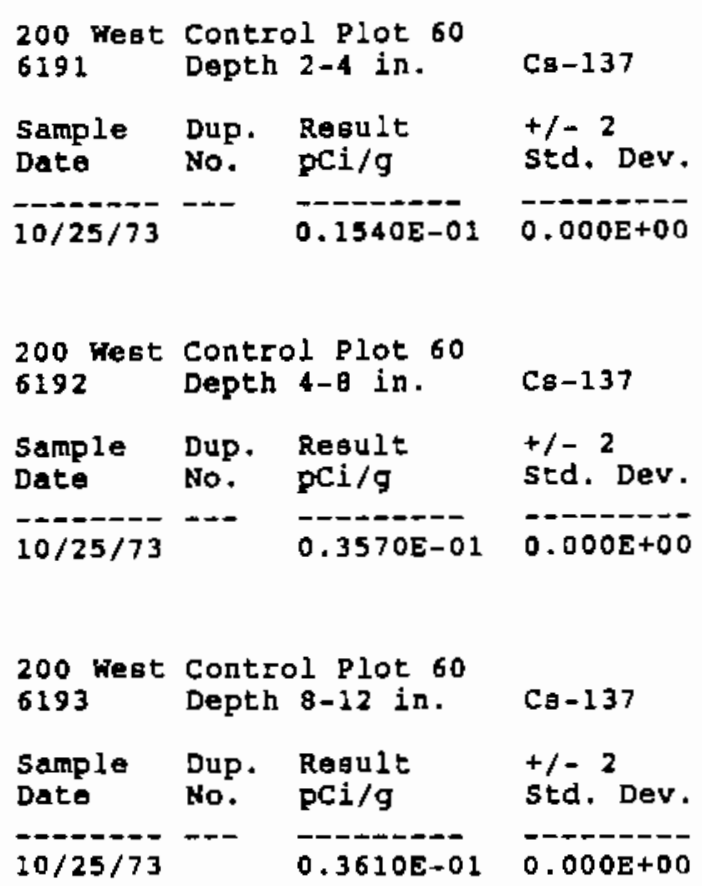

Other 200 Area Iocations

\begin{tabular}{|c|c|c|c|}
\hline $\begin{array}{l}200 \text { Area } \\
6021\end{array}$ & $\begin{array}{l}\text { Fire } \\
\text { Depth }\end{array}$ & $\begin{array}{c}\text { Station } \\
0-1 \text { in. }\end{array}$ & $\mathrm{Cs}-137$ \\
\hline $\begin{array}{l}\text { Sample } \\
\text { Date }\end{array}$ & $\begin{array}{l}\text { Dup. } \\
\text { No. }\end{array}$ & $\begin{array}{l}\text { Result } \\
\mathrm{pCi} / \mathrm{g}\end{array}$ & $\begin{array}{l}+/-2 \\
\text { std. Dev. }\end{array}$ \\
\hline $\begin{array}{l}10 / 25 / 73 \\
10 / 25 / 72 \\
09 / 30 / 71\end{array}$ & -- & $\begin{array}{l}0.6140 \mathrm{E}+00 \\
0.2370 \mathrm{E}+00 \\
0.6090 \mathrm{E}+00\end{array}$ & $\begin{array}{l}0.000 \mathrm{E}+00 \\
0.000 \mathrm{E}+00 \\
0.000 \mathrm{E}+00\end{array}$ \\
\hline $\begin{array}{l}200 \text { Area } \\
6044\end{array}$ & $\begin{array}{l}\text { Fire } \\
\text { Depth }\end{array}$ & $\begin{array}{l}\text { station } \\
1-2 \text { in. }\end{array}$ & $\mathrm{C}_{9} \rightarrow 137$ \\
\hline $\begin{array}{l}\text { Sample } \\
\text { Date }\end{array}$ & $\begin{array}{l}\text { Dup. } \\
\text { No. }\end{array}$ & $\begin{array}{l}\text { Result } \\
\text { pCi/g }\end{array}$ & $\begin{array}{l}+/-2 \\
\text { std. Dev. }\end{array}$ \\
\hline $\begin{array}{l}10 / 25 / 72 \\
09 / 30 / 71\end{array}$ & --- & $\begin{array}{l}0.2900 \mathrm{E}+00 \\
0.1260 \mathrm{E}+00\end{array}$ & $\begin{array}{l}0.000 \mathrm{E}+00 \\
0.000 \mathrm{E}+00\end{array}$ \\
\hline
\end{tabular}


TABLE A.3. (cont $d$ )

other 200 Acea Locations

Gable Pond Control Plot 43

\begin{tabular}{|c|c|c|c|}
\hline $\begin{array}{l}\text { Sample } \\
\text { Date }\end{array}$ & $\begin{array}{l}\text { Dup. } \\
\text { No. }\end{array}$ & $\begin{array}{l}\text { Regult } \\
\text { pCi/g }\end{array}$ & $\begin{array}{l}+/-2 \\
\text { std. Dev. }\end{array}$ \\
\hline $08 / 11 / 76$ & -- & $0.9120 \mathrm{E}+00$ & $0.000 \mathrm{E}+00$ \\
\hline $\begin{array}{l}\text { Inter. } 45 \\
6223\end{array}$ & 5 and & $\begin{array}{l}\text { Atruy Loop Rd. } \\
0-1 \text { in. }\end{array}$ & $\mathrm{CB}-137$ \\
\hline $\begin{array}{l}\text { Sample } \\
\text { Date }\end{array}$ & $\begin{array}{l}\text { Dup. } \\
\text { No. }\end{array}$ & $\begin{array}{l}\text { Result } \\
\mathrm{pCi} / \mathrm{g}\end{array}$ & $\begin{array}{l}+/-2 \\
\text { std. Dev. }\end{array}$ \\
\hline $\begin{array}{l}---1--- \\
08 / 10 / 76 \\
08 / 18 / 75 \\
09 / 24 / 74\end{array}$ & -- & $\begin{array}{l}0.1790 \mathrm{E}+00 \\
0.1700 \\
0.5760 \mathrm{E}+00 \\
0.7190 \mathrm{E}+00\end{array}$ & $\begin{array}{l}0.000 \mathrm{E}+00 \\
0.000 \mathrm{E}+00 \\
0.000 \mathrm{E}+00\end{array}$ \\
\hline
\end{tabular}

Army Loop Rd. Contl. Plot 40

\begin{tabular}{llll}
6226 & Depth $0-1$ in. & Ca-137 \\
Sample & Dup. Result & $+/-2$ \\
Date & No. & pCi $/ g$ & Std. Dev. \\
\hdashline $09 / 24 / 74$ & & $0.1680 E+00$ & $0.000 E+00$
\end{tabular}

Highray 240 Control Plot 54

$\begin{array}{llll}6002 & \text { Depth } 0-1 \text { in. } & \mathrm{Cs}-137 \\ & & & \\ \text { Sample } & \text { Dup. } & \text { Result } & +/-2 \\ \text { Date } & \text { No. } & \text { pCi/g } & \text { std. Dev. } \\ -09 / 23 / 74 & & 0.3660 \mathrm{E}+00 & 0.000 \mathrm{E}+00 \\ 10 / 25 / 73 & & 0.1820 \mathrm{E}+00 & 0.000 \mathrm{E}+00 \\ 10 / 25 / 72 & & 0.5070 \mathrm{E}+00 & 0.000 \mathrm{E}+00 \\ 09 / 29 / 71 & & 0.3290 \mathrm{E}+00 & 0.000 \mathrm{E}+00\end{array}$

Highway 240 Control Plot 54

$\begin{array}{llll}6025 & \text { Depth } & 1-2 \text { in. } & \text { Cs-137 } \\ \text { Sample } & \text { Dup. } & \text { Result } & +/-2 \\ \text { Date } & \text { No. } & \text { pCi/g } & \text { Std, Dev. } \\ -10 / 25 / 72 & & 0.1890 E+00 & 0.000 E+00 \\ 09 / 29 / 71 & & 0.5980 E-01 & 0.0005+00\end{array}$

300 Area

\begin{tabular}{|c|c|c|c|}
\hline $\begin{array}{l}\text { North of } \\
6322\end{array}$ & $\begin{array}{l}300 \mathrm{AI} \\
\text { Depth }\end{array}$ & $0-1$ in. & $\mathrm{Cs}-137$ \\
\hline $\begin{array}{l}\text { Sample } \\
\text { Date }\end{array}$ & $\begin{array}{l}\text { Dup. } \\
\text { No. }\end{array}$ & $\begin{array}{l}\text { Result } \\
\mathrm{pCi} / \mathrm{g}\end{array}$ & $\begin{array}{l}+/-2 \\
\text { std. Dev. }\end{array}$ \\
\hline $\begin{array}{l}-1-5 \\
04 / 29 / 87 \\
11 / 25 / 86 \\
04 / 29 / 86 \\
05 / 31 / 85 \\
05 / 16 / 84 \\
08 / 11 / 83 \\
09 / 03 / 82 \\
08 / 10 / 76\end{array}$ & -- & $\begin{array}{l}0.1170 \mathrm{E}+01 \\
0.4180 \mathrm{E}+00 \\
0.6680 \mathrm{E}+00 \\
0.4600 \mathrm{E}+00 \\
0.4310 \mathrm{E}+00 \\
0.5290 \mathrm{E}+00 \\
0.8500 \mathrm{E}+00 \\
0.4800 \mathrm{E}+00\end{array}$ & $\begin{array}{l}0.616 \mathrm{E}-01 \\
0.346 \mathrm{E}-01 \\
0.46 \mathrm{BE}-01 \\
0.437 \mathrm{E}-01 \\
0.375 \mathrm{E}-01 \\
0.412 \mathrm{E}-01 \\
0.516 \mathrm{E}-01 \\
0.000 \mathrm{0}+00\end{array}$ \\
\hline $\begin{array}{l}\text { North Cen } \\
6279\end{array}$ & $\begin{array}{l}\text { Ater } 30 \\
\text { Depth }\end{array}$ & $\begin{array}{l}\text { o Area Fence } \\
0-1 \text { in. }\end{array}$ & $\mathrm{Cs}-137$ \\
\hline $\begin{array}{l}\text { Sample } \\
\text { Date }\end{array}$ & $\begin{array}{l}\text { Dup. } \\
\text { No. }\end{array}$ & $\begin{array}{l}\text { Result } \\
\text { pci/g }\end{array}$ & $\begin{array}{l}+/-2 \\
\text { std. Dev. }\end{array}$ \\
\hline $08 / 20 / 75$ & + & $0.5920 \mathrm{E}+00$ & $0.0008+00$ \\
\hline
\end{tabular}

300 Area South Gate

6222 Depth o-1 in. Cs-137

\begin{tabular}{llll} 
Sample & Dup. & Result & $+/{ }^{2}$ \\
Date & No. & pCi/g & Std. Dev. \\
\hline $09 / 24 / 74$ & & $0.1500 \mathrm{E}+01$ & $0.000 \mathrm{E}+00$
\end{tabular}

South of 300 Area

6323 Depth $0-1$ in. Cg-137

Sample Dup. Result $+/-2$

Date No. pCi/g std. Dev.

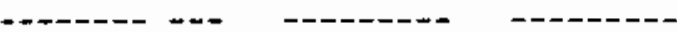

$04 / 29 / 86 \quad 0.6810 \mathrm{E}+00 \quad 0.434 \mathrm{E}-01$

$05 / 31 / 85 \quad 0.8 B 30 E+00 \quad 0.490 E-01$

$05 / 16 / 84 \quad 0.1090 \mathrm{E}+01 \quad 0.584 \mathrm{E}-01$

$08 / 11 / 83 \quad 0.1100 E+01 \quad 0.621 \mathrm{E}-01$

$09 / 03 / 82 \quad 0.1090 E+01 \quad 0.582 \mathrm{E}-01$

$08 / 10 / 76 \quad 0.5420 E+00 \quad 0.000 E+00$ 
TABLE A.3. (contd)

300 Area

\begin{tabular}{llll}
331 Bldg. Control Plot 50 & \\
6014 & Depth 0-1 in. & Cs-137 \\
Sample & Dup. Result & $+/-2$ \\
Date & No. & pCi/g & Std. Dev. \\
\hline $00 / 20 / 75$ & & $0.5700 \mathrm{E}-01$ & $0.000 \mathrm{E}+00$ \\
$10 / 25 / 73$ & $0.4450 \mathrm{E}-01$ & $0.000 \mathrm{E}+00$ \\
$10 / 25 / 72$ & $0.1460 \mathrm{E}+00$ & $0.000 \mathrm{E}+00$ \\
$09 / 30 / 71$ & & $0.1290 \mathrm{E}+01$ & $0.000 \mathrm{E}+00$
\end{tabular}

\begin{tabular}{llll}
$\begin{array}{l}\text { Wye Barricade (QC Dupl. A) } \\
\text { O816 } \\
\text { Depth } 0-1 \text { in. }\end{array}$ & Cs-137 \\
Sample & Dup. Result & $+/-2$ \\
Date & No. & pCi/g & Std. Dev. \\
\hline $04 / 29 / 87$ & & $0.3490 E+00$ & $0.321 E-01$ \\
$04 / 29 / 86$ & $0.5210 E-01$ & $0.169 E-01$ \\
$07 / 03 / 85$ & $0.8540 E+00$ & $0.461 E-01$ \\
$06 / 06 / 84$ & $0.2320 E+00$ & $0.256 E-01$ \\
$08 / 11 / 83$ & $0.7720 E+00$ & $0.523 E-01$ \\
$08 / 16 / 82$ & $0.1160 E+01$ & $0.830 E-01$
\end{tabular}

331 Bldg. Control plot 50

\begin{tabular}{llll}
6037 & Depth $1-2$ in. & Cs-137 \\
Sample & Dup. & Result & $+/-2$ \\
Date & No. & pCi/g & std. Dev. \\
\hline $10 / 25 / 72$ & & $0.2460 \mathrm{E}+00$ & $0.000 \mathrm{E}+00$ \\
$09 / 30 / 71$ & & $0.2040 \mathrm{E}+00$ & $0.000 \mathrm{E}+00$
\end{tabular}

400 Area and Vicinity

Wye Barricade

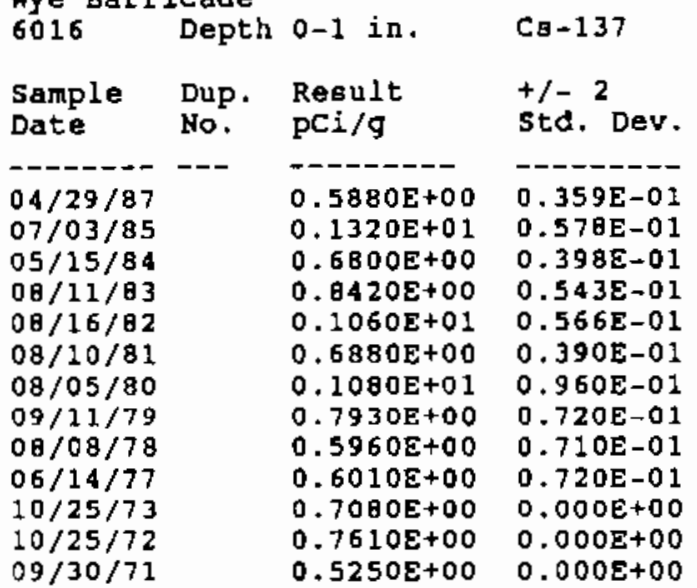

Wye Barricade ( $Q C$ Dupl. B)

0817 Depth o-1 in. Cs-137

Sample Dup. Reault t/- 2

Date No. $\mathrm{pCi} / \mathrm{g}$ std. Dev.

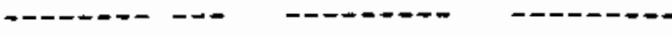

$04 / 29 / 87 \quad 0.5360 E+00 \quad 0.442 E-01$

$04 / 29 / 86 \quad 0.3260 E-01 \quad 0.134 E-01$

$07 / 03 / 85 \quad 0.9850 \mathrm{E}+00 \quad 0.489 \mathrm{E}-01$

$06 / 06 / 84 \quad 0.3120 E+00 \quad 0.344 E-01$

$08 / 11 / 83 \quad 0.6850 \mathrm{E}+00 \quad 0.526 \mathrm{E}-01$

$08 / 16 / 82 \quad 0.1140 E+01 \quad 0.925 E-01$

1 Mile $\mathrm{N}$ of WPPSS No 2

6324 Depth $0-1$ in. Cs-137

$\begin{array}{llll}\text { Sample } & \text { Dup, } & \text { Regult } & +/-2 \\ \text { Date } & \text { No. } & \text { pCi/g } & \text { Std. Dev. } \\ -08 / 10 / 76 & & <-.1580 \mathrm{E}-01 & 0.000 \mathrm{E}+00\end{array}$

1 Mile $s$ of WPPSS No. 2

$\begin{array}{llll}6325 & \text { Depth } 0-1 \text { in. } & \text { Cs-137 } \\ \text { Sample } & \text { Dup. Regult } & +/-2 \\ \text { Date } & \text { No. } & \text { pCi/g } & \text { Std. Dev. } \\ \text { OB/10/76 } & & 0.4740 \mathrm{E}+00 & 0.000 \mathrm{E}+00\end{array}$

\begin{tabular}{llll}
$\begin{array}{l}\text { Wye garricade } \\
6039 \\
\text { Depth } 1-2 \text { in. }\end{array}$ & Cs-137 \\
Sample & Dup. & Result & $+/-2$ \\
Date & No. & pCi/g & Std. Dev. \\
\hdashline $10 / 25 / 72$ & & $0.4160 \mathrm{E}+00$ & $0.000 \mathrm{E}+00$ \\
$09 / 30 / 71$ & & $0.2940 \mathrm{E}+00$ & $0.000 \mathrm{0}+00$
\end{tabular}


TABLE A. 3. (contd)

soo area and vicinity

$\begin{array}{llll}\text { FFTs Control Plot } 62 & \\ 6015 & \text { Depth } 0-1 \text { in. } & \text { C8-137 } \\ & & & +/-2 \\ \text { Sample } & \text { Dup. } & \text { Result } & +1 \\ \text { Date } & \text { No. } & \text { pC1/g } & \text { std. Dev. } \\ -09 / 24 / 74 & & 0.1360 \mathrm{E}+00 & 0.000 \mathrm{E}+00 \\ 10 / 25 / 73 & & 0.2640 \mathrm{E} \$ 0 & 0.000 \mathrm{E}+00 \\ 10 / 25 / 72 & & 0.1310 \mathrm{E}+00 & 0.000 \mathrm{E}+00 \\ 09 / 30 / 7 \mathrm{I} & & 0.1940 \mathrm{E}+00 & 0.000 \mathrm{E}+00\end{array}$

FFTF Control Plot 62

6038 Depth $1-2$ in. Cg-137

Sample Dup, Result $\quad+/-2$

Date No. pCi/g std. Dev.

$\overline{10 / 25 / 72}-0.5200 \mathrm{E}-01000$

$09 / 30 / 71 \quad 0.2350 E-01 \quad 0.000 E+00$

$\begin{array}{llll}\text { SE Side of FFTF } & \\ 6277 & \text { Depth } 0-1 \text { in. } & \text { Cs-137 } \\ \text { Sample } & \text { Dup. } & \text { Result } & +/-2 \\ \text { Date } & \text { No. } & \text { pCi/g } & \text { Std. Dev. } \\ -04 / 29 / 86 & & 0.4440 E-01 & 0.169 \mathrm{E}-01 \\ 06 / 05 / 85 & 0.8300 \mathrm{E}-01 & 0.212 \mathrm{E}-01 \\ 05 / 17 / 84 & 0.8010 \mathrm{E}-01 & 0.194 \mathrm{E}-01 \\ 08 / 12 / 83 & 0.1980 \mathrm{E}+00 & 0.309 \mathrm{E}-01 \\ 08 / 30 / 82 & 0.1940 \mathrm{E}+00 & 0.300 \mathrm{E}-01 \\ 08 / 10 / 81 & 0.7840 \mathrm{E}-01 & 0.170 \mathrm{E}-01 \\ 08 / 05 / 80 & 0.4020 \mathrm{E}+00 & 0.530 \mathrm{E}-01 \\ 09 / 11 / 79 & 0.1900 \mathrm{E}+00 & 0.430 \mathrm{~B}-01 \\ 08 / 08 / 78 & 0.3780 \mathrm{E}+00 & 0.580 \mathrm{E}-01 \\ 06 / 14 / 77 & 0.5190 \mathrm{E}+00 & 0.680 \mathrm{E}-01 \\ 08 / 20 / 75 & 0.1770 \mathrm{E}+00 & 0.000 \mathrm{E}+00\end{array}$

\begin{tabular}{llll}
0.5 Hile NE of FFTF & \\
6282 & Depth $0-1$ in. & Cs-137 \\
Sample & Dup. & Result & $+/-2$ \\
Date & No. & pCi/g & std. Dev. \\
\hline $04 / 29 / 87$ & & $0.3250 E+00$ & $0.326 E-01$ \\
$06 / 05 / 85$ & & $0.8030 E-01$ & $0.272 E-01$ \\
$05 / 17 / 84$ & $0.1170 E+00$ & $0.243 E-01$ \\
$08 / 12 / 83$ & $0.1770 E+00$ & $0.273 E-01$ \\
$08 / 30 / 82$ & $0.1260 E+00$ & $0.230 E-01$ \\
$08 / 10 / 81$ & $0.1050 E+00$ & $0.180 E-01$ \\
$08 / 05 / 80$ & $0.2170 E+00$ & $0.410 E-01$ \\
$09 / 11 / 79$ & $0.1880 E+00$ & $0.390 E-01$ \\
$0 B / 08 / 78$ & $0.1370 E+00$ & $0.370 E-01$ \\
$06 / 14 / 77$ & $0.1680 E+01$ & $0.120 E+00$ \\
$08 / 10 / 76$ & $0.6270 E+00$ & $0.000 E+00$ \\
$08 / 20 / 75$ & $0.1960 E-01$ & $0.000 E+00$
\end{tabular}

other onsite Locations

$\begin{array}{llll}\begin{array}{l}\text { N End of } \\ 6005\end{array} & \begin{array}{l}\text { Vernita Bridge } \\ \text { Depth } 0-1 \text { in. }\end{array} & \text { Cs-137 } \\ & & & \\ \text { Sample } & \text { Dup. } & \text { Regult } & +/ 2 \\ \text { Date } & \text { No. } & \text { pCi/g } & \text { Std. Dev. } \\ - & - & - \\ 05 / 02 / 86 & & 0.2000 E+00 & 0.349 E-01 \\ 06 / 11 / 85 & 0.4550 E+00 & 0.369 E-01 \\ 05 / 31 / 84 & 0.2650 E+00 & 0.341 E-01 \\ 08 / 11 / 83 & 0.5530 E+00 & 0.478 E-01 \\ 08 / 24 / 82 & 0.5800 E+00 & 0.651 E-01 \\ 09 / 23 / 74 & 0.9860 E-01 & 0.000 E+00 \\ 10 / 25 / 73 & 0.3800 E+00 & 0.000 E+00 \\ 10 / 25 / 72 & 0.2630 E+00 & 0.000 E+00 \\ 09 / 29 / 71 & 0.4780 E+00 & 0.000 E+00\end{array}$

\begin{tabular}{|c|c|c|c|}
\hline $\begin{array}{l}\text { N End of } \\
6028\end{array}$ & $\begin{array}{l}\text { Vernit } \\
\text { Depth }\end{array}$ & $\begin{array}{l}\text { a Bridge } \\
\text { 1-2 in. }\end{array}$ & $\mathrm{Cs}-137$ \\
\hline $\begin{array}{l}\text { Sample } \\
\text { Date }\end{array}$ & $\begin{array}{l}\text { Dup. } \\
\text { No. }\end{array}$ & $\begin{array}{l}\text { Result } \\
\text { pCi/g }\end{array}$ & $\begin{array}{l}+/{ }^{2} \\
\text { std. Dev. }\end{array}$ \\
\hline $\begin{array}{l}10 / 25 / 72 \\
09 / 29 / 71\end{array}$ & --- & $\begin{array}{l}0.1720 \mathrm{E}+00 \\
0.8130 \mathrm{E}-01\end{array}$ & $0.000 \mathrm{E}+00$ \\
\hline
\end{tabular}


TABLE A.3. (contd)

Other Onsite Locations

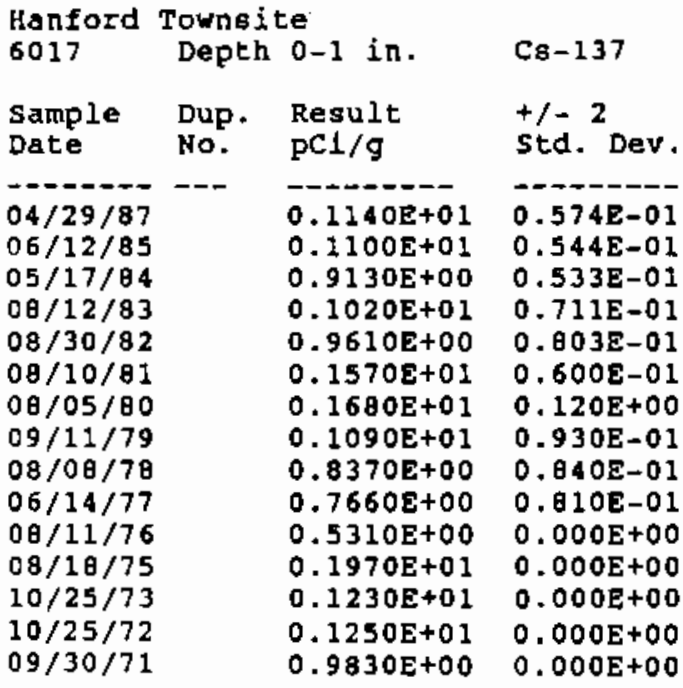

Hanford Townite

$\begin{array}{llll}6040 & \text { Depth } 1-2 \text { in. } & \text { C5-137 } \\ \text { Sample } & \text { Dup. Result } & +/-2 \\ \text { Date } & \text { No. } & \text { pC1/g } & \text { std. Dey. } \\ -10 / 25 / 72 & & 0.3110 \mathrm{E}+00 & 0.000 \mathrm{E}+00 \\ 09 / 30 / 71 & & 0.4120 \mathrm{E}-01 & 0.000 \mathrm{E}+00\end{array}$

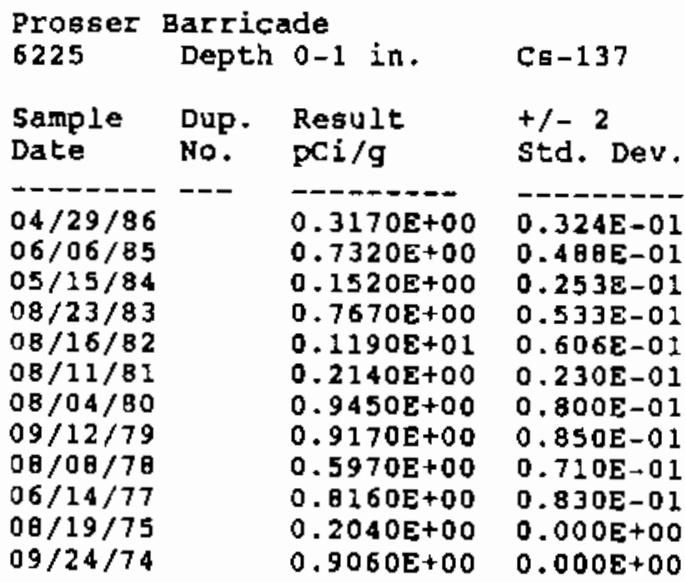

ERC (Emer. Reloc. Center)

6001 Depth $0-1$ in.

Cs-137

Sample Dup. Result

Date No. $\mathrm{pCi} / \mathrm{g}$

$+/-2$

$09 / 24 / 74$

$10 / 25 / 73$

No.

- -

$0.1060 \mathrm{E}+00$

$0.1260 \mathrm{E}+01$

$0.4200 \mathrm{E}-01$

$0.8030 \mathrm{E}+00$

std. Dey.

$0.000 \mathrm{E}+00$

$0.000 \mathrm{E}+00$

$0.000 E+00$

$09 / 30 / 71$

$0.000 E+00$

ERC (Emer. Reloc. Center)

6024 Depth 1-2 in.

Cg-137

Sample Dup. Result

Date No. $\mathrm{pCi} / \mathrm{g}$

$+/-2$

std. Dev.

$10 / 25 / 72$

No.

$0.3000 \mathrm{E}-02$

$-1-2-1$

$09 / 30 / 71$

$0.6430 \mathrm{E}-01$

$0.000 \mathrm{E}+00$

$E$ of Arid Land Ecol, Lab 6278 Depth $0-1$ in.

Ca -137

$\begin{array}{lll}\text { Sample } & \text { Dup, Result } \\ \text { Date } & \text { No. } & \text { pCi/g }\end{array}$

$05 / 20 / 87$

$0.1080 \mathrm{E}+01$

$06 / 12 / 85$

$0.1560 E+01$

$0.54808+00$

$0.1480 \mathrm{E}+01$

$0.1130 \mathrm{E}+01$

$0.3690 \mathrm{E}+00$

$0.1180 E+01$

$0.1070 E+01$

$0.6060 E+00$

$0.9730 \mathrm{E}+00$

$0.3950 \mathrm{E}+00$

$+1-2$

Std. Dev.

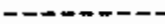

$0.623 \mathrm{E}-01$

$0.68 \theta \mathrm{E}-01$

$0.427 \mathrm{E}-01$

$0.689 \mathrm{E}-01$

$0.103 \mathrm{E}+00$

$0.290 E-01$

$0.100 E+00$

$12 / 7$

$0.930 E-01$

$0.690 \mathrm{E}-01$

$06 / 14 / 77$

$08 / 10 / 76$

$0.1550 \mathrm{E}+01$

$00 / 20 / 75$

$0.000 E+00$

$0.000 \mathrm{E}+00$ 
TABLE A.3. (contd)

other Onsite_focations

\begin{tabular}{llll}
$\begin{array}{l}\text { Rattlesnake Springs } \\
6003\end{array}$ & Depth & $0-1$ in. & Cs-137 \\
Sample & Dup. & Result & $+/-2$ \\
Date & No. & pCi/g & std. Dev. \\
\hline $05 / 07 / 87$ & & $0.3190 \mathrm{E}+00$ & $0.352 \mathrm{E}-01$ \\
$05 / 23 / 86$ & $0.3700 \mathrm{E}+00$ & $0.352 \mathrm{E}-01$ \\
$06 / 06 / 85$ & $0.4630 \mathrm{E}+00$ & $0.36 \mathrm{E}-01$ \\
$05 / 24 / 84$ & $0.1400 \mathrm{E}+00$ & $0.306 \mathrm{E}-01$ \\
$08 / 11 / 83$ & $0.5170 \mathrm{E}+00$ & $0.454 \mathrm{E}-01$ \\
$09 / 02 / 82$ & $0.7010 \mathrm{E}+00$ & $0.501 \mathrm{E}-01$ \\
$09 / 23 / 74$ & $0.9550 \mathrm{E}+00$ & $0.000 \mathrm{E}+00$ \\
$10 / 25 / 73$ & $0.1470 \mathrm{E}+01$ & $0.000 \mathrm{E}+00$ \\
$10 / 25 / 72$ & $0.1520 \mathrm{E}+01$ & $0.000 \mathrm{E}+00$ \\
$09 / 29 / 71$ & $0.9690 \mathrm{E}+00$ & $0.000 \mathrm{E}+00$
\end{tabular}

\begin{tabular}{llll} 
Yakima Barricade & \\
6027 & Deptb $1-2$ in. & Cs-137 \\
& & & $+/-2$ \\
Sanple Dup. Result & Std. Dev. \\
Date & No. & pCi/g & - \\
\hdashline $10 / 25 / 72$ & $0.3570 \mathrm{E}+00$ & $0.000 \mathrm{E}+00$ \\
$09 / 30 / 71$ & $<0.0000 \mathrm{E}+00$ & $0.000 \mathrm{E}+00$
\end{tabular}

Rattlesnake Springs

\begin{tabular}{llll}
6026 & Depth $1-2$ in. & Cs-137 \\
Sample & Dup. & Result & $+/-2$ \\
Date & No. & pCilg & Std. Dev. \\
\hline $10 / 25 / 72$ & & $0.3510 \mathrm{E}+00$ & $0.000 \mathrm{E}+00$ \\
$09 / 29 / 71$ & & $0.1360 \mathrm{E}+00$ & $0.000 \mathrm{E}+00$
\end{tabular}

Yakima Barricade

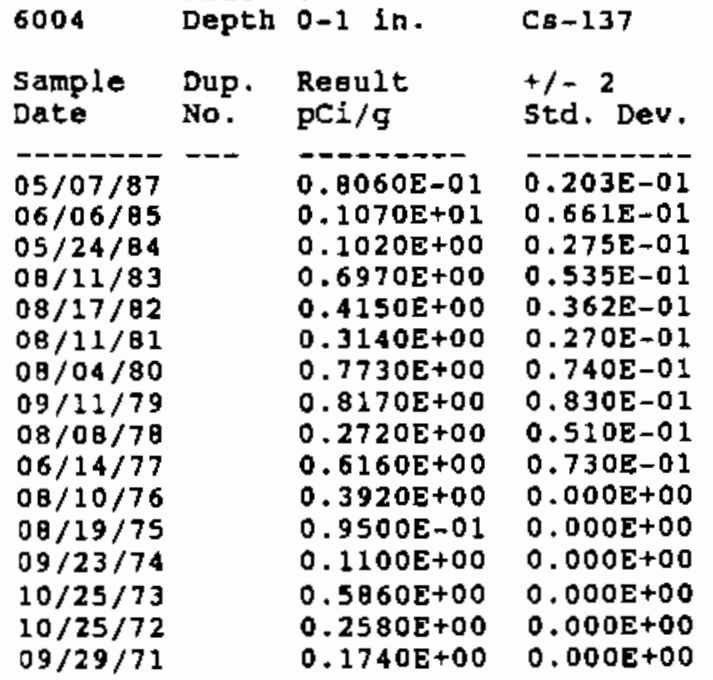


TABLE A.4. Plutonium-238 Concentrations in Soil at Onsite Locations

100 Areas

NE Corner of $100 \mathrm{~N}$ Area

\begin{tabular}{llll}
6275 & Depth $0-1$ in. & Pu-238 \\
Sample & Dup. Result & $+/-2$ \\
Date & No. & pCi/g & std. Dev. \\
\hdashline$-08 / 20 / 75$ & & $0.1820 \mathrm{E}-02$ & $0.000 \mathrm{E}+00$
\end{tabular}

1 Hile $\mathrm{NE}$ of $100 \mathrm{~N}$ Area

0590 Depth 0-1 in.

\begin{tabular}{llll} 
Sample & Dup. & Result & $+/-2$ \\
Date & No. & pCi/g & std. Dev. \\
\hline $04 / 30 / 86$ & & $0.3790 \mathrm{E}-03$ & $0.219 \mathrm{E}-03$ \\
$06 / 24 / 85$ & & $<0.1530 \mathrm{E}-03$ & $0.1 \mathrm{BgE}-03$ \\
$05 / 17 / 84$ & & $0.35 \mathrm{~B} 0 \mathrm{E}-03$ & $0.354 \mathrm{E}-03$ \\
$08 / 03 / 93$ & $<0.2730 \mathrm{E}-03$ & $0.385 \mathrm{E}-03$ \\
$08 / 24 / 82$ & & $0.1420 \mathrm{E}-02$ & $0.113 \mathrm{E}-02$
\end{tabular}

1 Mile $\mathrm{E}$ of $100 \mathrm{~N}$ Area 0578 Depth $0-1$ in.

\begin{tabular}{|c|c|c|c|}
\hline ample & $\begin{array}{l}\text { Dup. } \\
\text { No. }\end{array}$ & $\begin{array}{l}\text { Result } \\
\text { pci/g }\end{array}$ & $\begin{array}{l}+/-2 \\
\text { std. Dev. }\end{array}$ \\
\hline $\begin{array}{l}-04 / 29 / 87 \\
04 / 30 / 86 \\
06 / 24 / 85 \\
05 / 17 / 84 \\
08 / 11 / 83 \\
08 / 24 / 82\end{array}$ & --- & $\begin{array}{r}\overline{0.6450 \mathrm{E}-03} \\
0.7500 \mathrm{E}-03 \\
0.2850 \mathrm{E}-03 \\
<0.4550 \mathrm{E}-03 \\
<0.31 \mathrm{0OE}-03 \\
\langle 0.5840 \mathrm{E}-03\end{array}$ & $\begin{array}{l}9 \mathrm{BE}-03 \\
53 \mathrm{E}-03 \\
87 \mathrm{E}-03 \\
43 \mathrm{E}-03 \\
37 \mathrm{E}-03 \\
6 \mathrm{E}-03\end{array}$ \\
\hline
\end{tabular}

1 Mile SE of $100 \mathrm{~N}$ Trench 6320 Depth $0-1 \mathrm{in}$.

$\mathrm{Pu}-23 \mathrm{\theta}$

\begin{tabular}{llll} 
Sample & Dup. & Regult & $+/-2$ \\
Date & No. & pCi/g & Std. Dev. \\
\hdashline $08 / 03 / 84$ & & $0.6900 \mathrm{E}-03$ & $0.368 E-03$ \\
$08 / 11 / 76$ & & $<-.1390 \mathrm{E}-03$ & $0.000 \mathrm{E}+00$
\end{tabular}

Fahluke slope Opposite 100-N

6006 Depth 0-1 in. Pu-238

\begin{tabular}{llll} 
Sample & Dup. & Result & $+/-2$ \\
Date & No. & pCi/g & Std. Dev. \\
\hline $09 / 23 / 74$ & & $0.0480 \mathrm{E}-03$ & $-0.000 \mathrm{E}+00$ \\
$10 / 25 / 73$ & & $<0.1560 \mathrm{E}-02$ & $0.300 \mathrm{E}-02$ \\
$10 / 25 / 72$ & & $0.1550 \mathrm{E}-02$ & $0.000 \mathrm{E}+00$ \\
$09 / 29 / 71$ & & $0.2500 \mathrm{E}-02$ & $0.000 \mathrm{E}+00$
\end{tabular}

\begin{tabular}{llll} 
Hahluke & slope Opposite $100-\mathrm{N}$ \\
6029 & Depth & 1-2 in. & Pu-23g \\
& & & \\
Sample & Dup. Result & $+/-2$ \\
Date & No. & pCi/g & Std. Dev. \\
\hline $10 / 25 / 72$ & & $0.6350 \mathrm{E}-02$ & $0.000 \mathrm{E}+00$ \\
$09 / 29 / 71$ & & $0.3000 \mathrm{E}-02$ & $0.000 \mathrm{E}+00$
\end{tabular}

\begin{tabular}{llll}
100 Area Fire station & \\
0580 & Depth $0-1$ in. & Pu-238 \\
& & \\
Sample & Dup. Regult & $+/-2$ \\
Date & No. & pCi/g & Std. Dev. \\
\hline $04 / 29 / 87$ & & $0.5540 E-03$ & $0.254 E-03$ \\
$04 / 30 / 86$ & $0.1200 E-02$ & $0.412 E-03$ \\
$05 / 30 / 85$ & $0.7050 E-03$ & $0.286 E-03$ \\
$05 / 17 / 84$ & $0.7410 E-03$ & $0.320 E-03$ \\
$08 / 05 / 83$ & $<-1000 \mathrm{E}-03$ & $0.500 \mathrm{E}-03$ \\
$08 / 24 / 82$ & $<0.1120 \mathrm{E}-03$ & $0.774 \mathrm{E}-03$
\end{tabular}

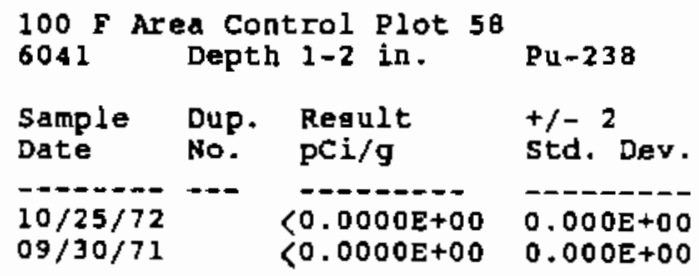


TABLE A.4. (contd)

200 East Area

\begin{tabular}{llll}
200 E Control Plot 59 & \\
6019 & $\begin{array}{l}\text { Depth } \\
0-3 \text { in. }\end{array}$ & Pu-238 \\
Sample & Dup. & Result & $+/-2$ \\
Date & No. & PCi/g & std. Dev. \\
\hline $08 / 18 / 75$ & & $-.4640 E-02$ & $0.000 \mathrm{E}+00$ \\
$09 / 24 / 74$ & & $0.1990 \mathrm{E}+00$ & $0.000 \mathrm{E}+00$ \\
$10 / 25 / 73$ & $0.2340 \mathrm{E}+00$ & $0.000 \mathrm{E}+00$ \\
$10 / 25 / 72$ & $0.2610 \mathrm{E}+00$ & $0.000 \mathrm{E}+00$ \\
$09 / 30 / 71$ & & $0.2860 \mathrm{E}-01$ & $0.000 \mathrm{E}+00$
\end{tabular}

200 E Control Plot 59

6042 Depth $1-2$ in.

\begin{tabular}{llll} 
Sample & Dup. & Result & $+/-2$ \\
Date & No. & pCi/g & Std. Dev. \\
\hline $10 / 25 / 73$ & & $<0.1820 \mathrm{E}-02$ & $0.300 \mathrm{E}-02$ \\
$10 / 25 / 72$ & & $<0.0000 \mathrm{E}+00$ & $0.000 \mathrm{E}+00$ \\
$09 / 29 / 71$ & & $0.2000 \mathrm{E}-02$ & $0.000 \mathrm{E}+00$
\end{tabular}

200 E Control Plot 59

6198 Depth 2-4 in

Sample Dup. Reoult

Date No. PCi/g

---0-0- --- - -

$10 / 25 / 73 \quad 0.8480 \mathrm{E}-02$
Pu-238

$+/-2$

std. Dev.
$0.300 \mathrm{E}-02$
200 ENC (East North Central)

6362

Depth $0-1$ in.

Sample

Dup. Result

No. $\mathrm{pCi} / \mathrm{g}$

Date

$\overline{05 / 07 / 87}$

$04 / 30 / 86$

$05 / 31 / 85$

$05 / 17 / 84$

$08 / 12 / 83$

$08 / 30 / 82$

$08 / 10 / 81$

$08 / 10 / 81$

$08 / 05 / 80$

$09 / 11 / 79$

$08 / 10 / 78$

$06 / 14 / 77$

$0.4310 \mathrm{E}-03$

$0.4300 \mathrm{E}-03$

$0.1150 \mathrm{E}-02$

$<0.6070 \mathrm{E}-03$

$0.1260 \mathrm{E}-02$

$<0.1250 \mathrm{E}-02$

$1<0.3460 \mathrm{E}-03$

$0.3080 \mathrm{E}-01$

$0.1720 E-02$

$0.1420 \mathrm{E}-02$

$<0.1190 \mathrm{E}-02$

$0.9180 \mathrm{E}-02$

PU -238

$+1-2$

std. Dev.

- - -

$0.268 \mathrm{E}-03$

$0.259 E-03$

$0.373 E-03$

$0.607 E-03$

$0.103 \mathrm{E}-02$

$0.176 \mathrm{E}-02$

$0.560 \mathrm{E}-03$

$0.210 E-02$

$0.600 \mathrm{E}-03$

$0.120 \mathrm{OE}-02$

$0.230 E-02$

$0.320 \mathrm{E}-02$

1.25 Mileg East of PUREX

0581 Deptb 0-1 In.

$\mathrm{Pu}-23 \mathrm{~B}$

Sample

Date

Dup

Result

No. $\mathrm{pCi} / \mathrm{g}$

$05 / 07 / 87$

$04 / 30 / 86$

$05 / 31 / 85$

$05 / 15 / 04$

$08 / 05 / 83$

$O B / 24 / 82$
$<0.12208-03$

$0.6410 \mathrm{E}-03$

$0.4030 \mathrm{E}-03$

$0.4890 E-03$

$<0.4340 \mathrm{E}-03$

$<-.7350 \mathrm{E}-03$
$+/-2$

std. Dev.

$0.213 E-03$

$0.340 \mathrm{E}-03$

$0.214 E-03$

$0.311 \mathrm{E}-03$

$0.434 \mathrm{E}-03$

$0.451 E-03$

\begin{tabular}{|c|c|c|c|}
\hline $\begin{array}{l}200 \mathrm{E} \\
6190\end{array}$ & Depth & 8-12 in. & $\mathrm{Pu}-23 \mathrm{~g}$ \\
\hline $\begin{array}{l}\text { Sample } \\
\text { Date }\end{array}$ & $\begin{array}{l}\text { Dup. } \\
\text { No. }\end{array}$ & $\begin{array}{l}\text { Result } \\
\mathrm{pCl} / \mathrm{g}\end{array}$ & $\begin{array}{l}+/-2 \\
\text { std. Dev. }\end{array}$ \\
\hline $10 / 25 / 73$ & -- & $0.4360 \mathrm{E}-02$ & $0.300 \mathrm{E}-02$ \\
\hline
\end{tabular}


TABLE A.4. (contd)

200 East_Area

200 ESE (East South East)

6022 Depth $0-1$ in.

$\mathrm{Pu}-238$

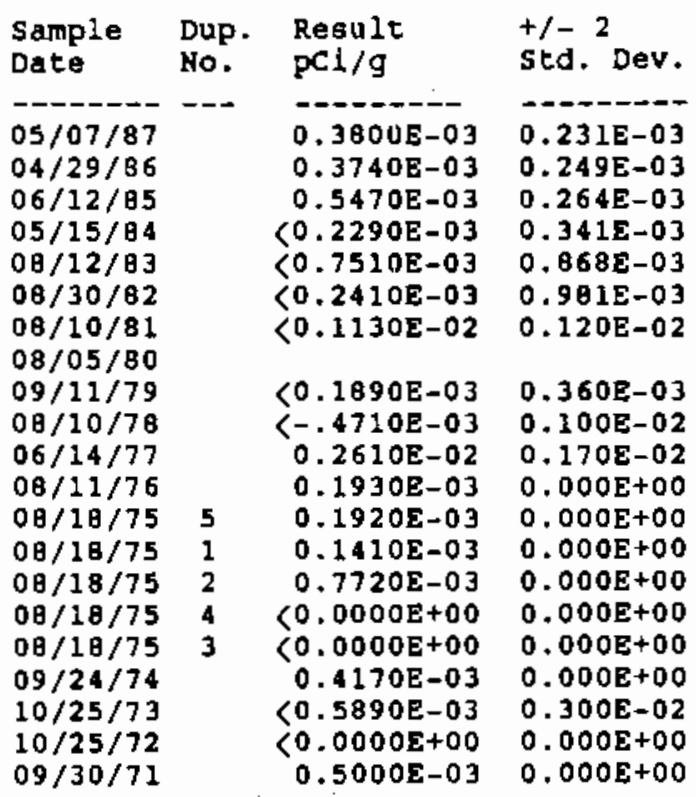

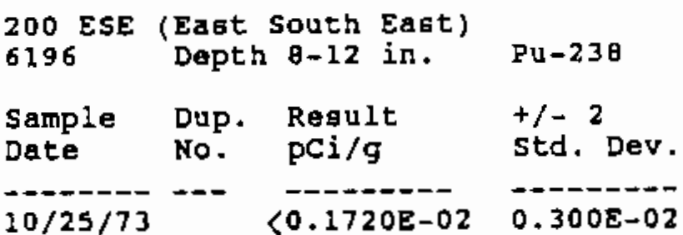

2 Milea South of PUREX

0582 Depth $0-1$ in.

Pu-238

\begin{tabular}{llll} 
Sample & Dup. & Result & $+1-2$ \\
Date & No. & pCi/g & Std. Dev. \\
\hline $05 / 07 / 87$ & & $<0.1010 \mathrm{E}-03$ & $0.125 \mathrm{E}-03$ \\
$04 / 30 / 86$ & $0.1700 \mathrm{E}-03$ & $0.139 \mathrm{E}-03$ \\
$06 / 24 / 85$ & $<0.1440 \mathrm{E}-03$ & $0.365 \mathrm{E}-03$ \\
$05 / 15 / 84$ & $<0.9090 \mathrm{E}-03$ & $0.151 \mathrm{E}-02$ \\
$08 / 05 / 83$ & $<0.1580 \mathrm{E}-03$ & $0.223 \mathrm{E}-03$ \\
$08 / 24 / 82$ & $<-.3400 \mathrm{E}-03$ & $0.389 \mathrm{E}-03$
\end{tabular}

3 Hiles SSW of PUREX

0583 Depth $0-1$ in. Pu-238

Sample Dup. Result $+/-2$

Date No. pCl/g std. Dev.

\begin{tabular}{|c|c|c|c|}
\hline 5045 & $\begin{array}{l}\text { (East } \\
\text { Depth }\end{array}$ & $\begin{array}{l}\text { South East) } \\
1-2 \text { in. }\end{array}$ & $P u-238$ \\
\hline $\begin{array}{l}\text { ample } \\
\text { ate }\end{array}$ & $\begin{array}{l}\text { Dup. } \\
\text { No. }\end{array}$ & $\begin{array}{l}\text { Result } \\
\text { pCi/g }\end{array}$ & $\begin{array}{l}+/-2 \\
\text { std. Dev. }\end{array}$ \\
\hline $\begin{array}{l}10 / 25 / 73 \\
10 / 25 / 72 \\
09 / 30 / 71\end{array}$ & --- & $\begin{array}{l}<0.3290 \mathrm{E}-03 \\
0.0020 \mathrm{E}-04 \\
0.1900 \mathrm{E}-02\end{array}$ & $\begin{array}{l}0.300 E-02 \\
0.000 E+00 \\
0.000 E+00\end{array}$ \\
\hline
\end{tabular}

$05 / 07 / 87-0<0$

$04 / 30 / 86 \quad\langle 0.2180 E-03$

$06 / 24 / 85 \quad 0.8860 E-03$

$05 / 15 / 84 \quad<0.4800 E-04$

$08 / 05 / 83 \quad 0.4280 \mathrm{E}-03$

$O B / 24 / B 2<-.1270 E-04$

$0.716 E-04$

$0.267 E-03$

$0.429 \mathrm{E}-03$

$0.551 \mathrm{E}-03$

$0.286 \mathrm{E}-03$

$0.277 \mathrm{E}-03$

\begin{tabular}{|c|c|c|c|}
\hline $\begin{array}{l}\text { Side } 0 \\
5281\end{array}$ & $\begin{array}{l}\text { f US E } \\
\text { Depth }\end{array}$ & cology & $P u-23 \theta$ \\
\hline $\begin{array}{l}\text { Sample } \\
\text { bate }\end{array}$ & $\begin{array}{l}\text { Dup. } \\
\text { No. }\end{array}$ & $\begin{array}{l}\text { Result } \\
\mathrm{pCi} / \mathrm{g}\end{array}$ & $\begin{array}{l}+/-2 \\
\text { std. Dev. }\end{array}$ \\
\hline $\begin{array}{l}----1--1 \\
08 / 03 / 84 \\
08 / 21 / 76 \\
08 / 18 / 75\end{array}$ & $-\infty$ & $\begin{array}{l}<0.3210 \mathrm{E}-03 \\
0.9740 \mathrm{E}-02 \\
0.3980 \mathrm{E}-03\end{array}$ & $\begin{array}{l}0.36 \mathrm{BE}-03 \\
0.000 \mathrm{E}+00 \\
0.000 \mathrm{E}+00\end{array}$ \\
\hline
\end{tabular}

200 ESE (East South East)

$\begin{array}{llll}6195 & \text { Depth } 4-8 \text { in. } & \text { Pu-238 } \\ \text { Sample } & \text { Dup. Regult } & +/-2 \\ \text { Date } & \text { No. } & \text { pCi/g } & \text { Std. Dev. } \\ -10 / 25 / 73 & & <0.5990 E-03 & 0.300 E-02\end{array}$


TABLE A.4. (contd)

200 Nest_Area

\begin{tabular}{|c|c|c|c|}
\hline of 200 & $\begin{array}{l}\text { West } \\
\text { Depth }\end{array}$ & $a_{0-1}^{a t e}$ in. & $P u-230$ \\
\hline Sample & $\begin{array}{l}\text { Dup. } \\
\text { No. }\end{array}$ & $\begin{array}{l}\text { Regult } \\
\mathrm{pCi} / \mathrm{g}\end{array}$ & $\begin{array}{l}+/{ }^{2} \\
\text { std. Dev. }\end{array}$ \\
\hline $\begin{array}{l}-0 .-1 / 0 \\
05 / 07 / 87 \\
05 / 01 / 86 \\
05 / 31 / 85 \\
05 / 15 / 84 \\
08 / 12 / 83 \\
08 / 30 / 82 \\
08 / 10 / 81 \\
08 / 04 / 80 \\
09 / 11 / 79 \\
08 / 09 / 78 \\
06 / 14 / 77 \\
08 / 11 / 76\end{array}$ & - - & $\begin{array}{l}-.-1 \\
0.2110 \mathrm{E}-02 \\
0.5270 \mathrm{E}-02 \\
0.5740 \mathrm{E}-02 \\
0.1400 \mathrm{E}-02 \\
0.9130 \mathrm{E}-02 \\
0.0190 \mathrm{E}-02 \\
0.4600 \mathrm{E}-02 \\
0.9790 \mathrm{E}-02 \\
0.7350 \mathrm{E}-02 \\
0.6130 \mathrm{E}-02 \\
0.8650 \mathrm{E}-02 \\
0.2000 \mathrm{E}-02 \\
0.5140 \mathrm{E}-02\end{array}$ & $\begin{array}{l}-0.486 \mathrm{E}-03 \\
0.757 \mathrm{E}-03 \\
0.914 \mathrm{E}-03 \\
0.572 \mathrm{E}-03 \\
0.286 \mathrm{E}-02 \\
0.181 \mathrm{E}-02 \\
0.910 \mathrm{E}-03 \\
0.210 \mathrm{E}-02 \\
0.220 \mathrm{E}-02 \\
0.240 \mathrm{E}-02 \\
0.450 \mathrm{E}-02 \\
0.000 \mathrm{E}+00 \\
0.000 \mathrm{E}+00\end{array}$ \\
\hline
\end{tabular}

200 Weat. Control Plot 60

6191 Depth 2-4 in. Pu-23日

Sample Dup. Result $+/-2$

Date No. pCilg std. Dev.

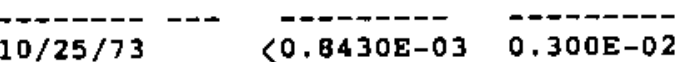

200 West Control Plot 60

6192 Depth 4-8 in. Pu-23日

Sample Dup. Reeult $+/-2$

Date No. pCi/g std. Dev.

--10/25/73 --- -

$10 / 25 / 73 \quad<0.6660 \mathrm{E}-03 \quad 0.300 \mathrm{E}-02$

200 West: Control Plot 60

6193 Depth 8-12 in. Pu-238

2 Miles $S$ of 200 West

$\begin{array}{llll}0584 & \text { Depth } 0-1 \text { in. } & \text { Pu-238 } \\ \text { Sample } & \begin{array}{l}\text { Dup. } \\ \text { Nesult }\end{array} & +/-2 \\ \text { Date } & \text { No. } & \text { pCi/g } & \text { std. Dev. } \\ -05 / 01 / 86 & & 0.3460 \mathrm{E}-03 & 0.216 \mathrm{E}-03 \\ 05 / 31 / 85 & & 0.1940 \mathrm{E}-03 & 0.170 \mathrm{E}-03 \\ 05 / 17 / 84 & <0.7740 \mathrm{E}-03 & 0.100 \mathrm{E}-02 \\ 08 / 05 / 83 & & 0.5680 \mathrm{E}-03 & 0.955 \mathrm{E}-03 \\ 08 / 24 / 82 & & <-.4520 \mathrm{E}-03 & 0.615 \mathrm{E}-03\end{array}$

\begin{tabular}{llll} 
Sample & Dup. & Result & $+/-2$ \\
Date & No. & pCi/g & Std. Dev. \\
\hline $10 / 25 / 73$ & & $<0.2440 \mathrm{O}-02$ & $0.300 \mathrm{0}-02$
\end{tabular}

other 200 srea tocations

200 Area Fire station

6021 Depth 0-1 in. 2u-238

200 West Control Plot 60

6020 Depth $0-1$ in.

Pu-238

Sample Dup. Result $+/-2$

Date No. pCi/g std. Dev.

-------- --D =-------

$08 / 18 / 75 \quad 0.3420 \mathrm{E}-04$

$09 / 24 / 74 \quad 0.1380 \mathrm{E}-02$

$10 / 25 / 73 \quad<0.2270 E-02$

$10 / 25 / 72 \quad<0.0000 \mathrm{E}+00$

$0.000 \mathrm{E}+00$

$0.000 \mathrm{E}+00$

$0.300 \mathrm{E}-02$

$0.000 E+00$

$09 / 30 / 71$

$0.000 E+00$

200 Weat Control Plot 60 6043 Depth 1-2 in.

Pu -238

\begin{tabular}{lll} 
Sample & Dup. & Result \\
Date & No. & pCi/g \\
\hline $10 / 25 / 73$ & & $<0.9400 \mathrm{E}-03$ \\
$10 / 25 / 72$ & $0.6560 \mathrm{E}-02$ \\
$09 / 30 / 71$ & & $<0.0000 \mathrm{E}+00$
\end{tabular}

$+1-2$

std. Dev.

------

$0.300 E-02$

$0.000 \mathrm{E}+00$

$0.000 \mathrm{E}+00$

\begin{tabular}{|c|c|c|c|}
\hline $\begin{array}{l}00 \text { Area } \\
044\end{array}$ & $\begin{array}{l}\text { Fire } \\
\text { Depth }\end{array}$ & $\begin{array}{l}\text { station } \\
1-2 \text { in. }\end{array}$ & $\mathrm{Pu}-238$ \\
\hline & $\begin{array}{l}\text { Dup. } \\
\text { No. }\end{array}$ & $\begin{array}{l}\text { Regult } \\
\mathrm{pCi} / \mathrm{g}\end{array}$ & $\begin{array}{l}+/-2 \\
\text { std. Dev. }\end{array}$ \\
\hline $\begin{array}{l}0 / 25 / 72 \\
9 / 30 / 7 \mathrm{~L}\end{array}$ & & $\begin{array}{l}<0.0000 \mathrm{E}+00 \\
0.3200 \mathrm{E}-02\end{array}$ & $\begin{array}{l}0.000 \mathrm{E}+00 \\
0.000 \mathrm{E}+00\end{array}$ \\
\hline
\end{tabular}


TABLE A.4. (contd)

other 200 Area tocations

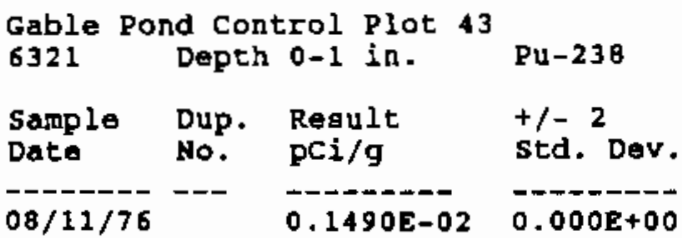

\begin{tabular}{|c|c|c|c|}
\hline $\begin{array}{l}\text { nter. } \\
223\end{array}$ & $\begin{array}{l}5 \text { and } \\
\text { Depth }\end{array}$ & $\begin{array}{c}\text { Army Loop R } \\
0-1 \text { in. }\end{array}$ & Pu-238 \\
\hline $\begin{array}{l}\text { Sample } \\
\text { Date }\end{array}$ & $\begin{array}{l}\text { Dup. } \\
\text { No. }\end{array}$ & $\begin{array}{l}\text { Result } \\
\mathrm{pCi} / \mathrm{g}\end{array}$ & $\begin{array}{l}+/-2 \\
\text { std. Dev. }\end{array}$ \\
\hline $\begin{array}{l}-08 / 10 / 76 \\
08 / 18 / 75 \\
09 / 24 / 74\end{array}$ & - & $\begin{array}{l}0.2040 \mathrm{E}-03 \\
0.2350 \mathrm{E}-04 \\
0.1110 \mathrm{E}-02\end{array}$ & $\begin{array}{l}0.000 \mathrm{E}+00 \\
0.000 \mathrm{E}+00 \\
0.000 \mathrm{E}+00\end{array}$ \\
\hline
\end{tabular}

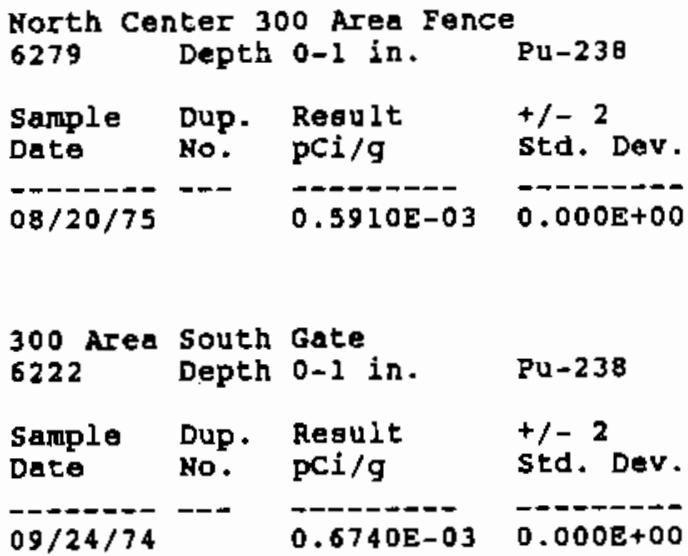

\begin{tabular}{llll}
$\begin{array}{l}\text { South of } \\
6323\end{array}$ & $\begin{array}{l}300 \text { Area } \\
\text { Depth } 0-1 \text { in. }\end{array}$ & Pu-238 \\
& & & \\
Sample & Dup. & Regult & $+/-2$ \\
Date & No. & pCi/g & std. Dev. \\
\hline $04 / 29 / 86$ & & $0.4320 E-03$ & $0.223 E-03$ \\
$05 / 31 / 85$ & & $0.3140 E-03$ & $0.199 E-03$ \\
$05 / 16 / 84$ & $0.3450 E-03$ & $0.310 E-03$ \\
$08 / 11 / 83$ & $0.1040 E-02$ & $0.302 E-03$ \\
$09 / 03 / 82$ & $<0.1030 E-04$ & $0.642 E-03$ \\
$08 / 10 / 76$ & & $0.3990 E-03$ & $0.000 E+00$
\end{tabular}

\begin{tabular}{llll} 
Highway & 240 Control plot 54 \\
6025 & Depth $1-2$ in. & Pu-238 \\
& & & \\
Sample & Dup. & Result & $+/-2$ \\
Date & No. & pCi/g & std. Dev. \\
\hline $10 / 25 / 72$ & & $0.4580 E-02$ & $0.000 E+00$ \\
$09 / 29 / 71$ & & $0.4000 E-02$ & $0.000 E+00$
\end{tabular}

\begin{tabular}{|c|c|c|c|}
\hline $\begin{array}{l}\text { North of } \\
6322\end{array}$ & $\begin{array}{l}300 \text { A } \\
\text { Depth }\end{array}$ & $e_{0-1}$ in. & $P u-238$ \\
\hline & $\begin{array}{l}\text { Dup. } \\
\text { No. }\end{array}$ & $\begin{array}{l}\text { Result } \\
\mathrm{pCi} / \mathrm{g}\end{array}$ & $\begin{array}{l}+/{ }^{2} \\
\text { std. Dev. }\end{array}$ \\
\hline $\begin{array}{l}-04 / 29 / 87 \\
041 / 25 / 86 \\
05 / 31 / 85 \\
05 / 16 / 84 \\
08 / 11 / 83 \\
09 / 03 / 82 \\
08 / 10 / 76\end{array}$ & -- & $\begin{array}{r}0.6500 \mathrm{E}-03 \\
<0.1530 \mathrm{E}-03 \\
0.2060 \mathrm{E}-03 \\
<0.0000 \mathrm{E}+00 \\
0.4310 \mathrm{E}-03 \\
0.3870 \mathrm{E}-02 \\
0.7110 \mathrm{E}-03\end{array}$ & $\begin{array}{l}0.277 \mathrm{E}-03 \\
0.252 \mathrm{E}-03 \\
0.165 \mathrm{E}-03 \\
0.000 \mathrm{E}+00 \\
0.352 \mathrm{E}-03 \\
0.172 \mathrm{E}-02 \\
0.000 \mathrm{E}+00\end{array}$ \\
\hline
\end{tabular}

300 Acea

\begin{tabular}{llll} 
Highway & 240 Control Plot 54 \\
6002 & Depth $0-2$ in. & Pu-238 \\
& & & \\
Sample & Dup, Result & $+/-2$ \\
Date & No. & pci/g & Std. Dev. \\
\hline $09 / 23 / 74$ & & $0.5960 E-03$ & $0.000 E+00$ \\
$10 / 25 / 73$ & $<0.1990 E-03$ & $0.300 E-02$ \\
$10 / 25 / 72$ & $0.3160 E-02$ & $0.000 E+00$ \\
$09 / 30 / 71$ & $0.5000 E-03$ & $0.000 E+00$ \\
$09 / 29 / 71$ & $<0.0000 E+00$ & $0.000 E+00$
\end{tabular}

\begin{tabular}{|c|c|c|c|}
\hline $\begin{array}{l}\text { Army LOO } \\
6226\end{array}$ & $\begin{array}{l}\text { Pd. } \\
\text { Depth }\end{array}$ & $\begin{array}{l}\text { Contl. Plot } \\
0-1 \text { in. }\end{array}$ & $\begin{array}{l}40 \\
P u-238\end{array}$ \\
\hline $\begin{array}{l}\text { Sample } \\
\text { Date }\end{array}$ & $\begin{array}{l}\text { Dup. } \\
\text { No. }\end{array}$ & $\begin{array}{l}\text { Result } \\
\text { pci/g }\end{array}$ & $\begin{array}{l}+/-2 \\
\text { std. Dev. }\end{array}$ \\
\hline $09 / 24 / 74$ & $m$ & $0.3020 \mathrm{E}-03$ & $0.000 \mathrm{E}+00$ \\
\hline
\end{tabular}


TABLE A.4. (contd)

300 Area

331 Bldg. Contral plat 50

\begin{tabular}{llll}
6014 & Depth $0-1$ in. & Pu-238 \\
Sample & Dup. & Result & $+/-2$ \\
Date & No. & pCi/g & std. Dev. \\
\hline $08 / 20 / 75$ & & $0.9090 \mathrm{E}-01$ & $0.000 \mathrm{E}+00$ \\
$10 / 25 / 73$ & & $<0.0000 \mathrm{E}+00$ & $0.000 \mathrm{E}+00$ \\
$10 / 25 / 72$ & & $<0.0000 \mathrm{E}+00$ & $0.000 \mathrm{E}+00$ \\
$09 / 30 / 71$ & & $0.0000 \mathrm{E}+00$ & $0.000 \mathrm{E}+00$
\end{tabular}

331 Bldg. Control plot 50

\begin{tabular}{llll}
6037 & Depth $1-2$ in. & Pu-238 \\
Sample & Dup. & Result & $+/-2$ \\
Date & No. & pCi/g & Std. Dev. \\
\hline $10 / 25 / 72$ & -- & $-0.1180 \mathrm{D}-01$ & $0.000 \mathrm{E}+00$ \\
$09 / 30 / 71$ & & $0.1000 \mathrm{D}-03$ & $0.000 \mathrm{E}+00$
\end{tabular}

400 Area and Vicinity

Wye Barricade

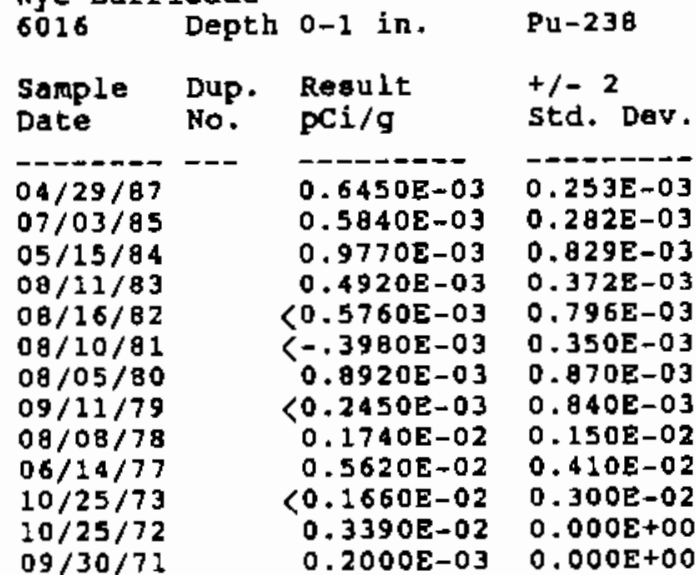

Wye Barricade

\begin{tabular}{llll}
6039 & Depth & $1-2$ in. & Pu-238 \\
& & & \\
Sample & Dup. & Result & $+/-2$ \\
Date & No. & pCi $/ g$ & std. Dev. \\
\hline $10 / 25 / 72$ & & $0.4770 \mathrm{E}-02$ & $0.000 \mathrm{E}+00$ \\
$09 / 30 / 71$ & & $0.0000 \mathrm{E}+00$ & $0.000 \mathrm{E}+00$
\end{tabular}

Wye Barricade (OC Dupl, A)

0816 Depth 0-1 in. Pu-238

\begin{tabular}{llll} 
Sample & Dup. & Result & $+/-2$ \\
Date & No. & pCi/g & Std. Dev. \\
\hdashline $04 / 29 / 87$ & & $0.4640 \mathrm{E}-03$ & $0.258 \mathrm{E}-03$ \\
$04 / 29 / 86$ & $<0.1620 \mathrm{E}-03$ & $0.18 \mathrm{BE}-03$ \\
$07 / 03 / 85$ & $0.3080 \mathrm{E}-03$ & $0.231 \mathrm{E}-03$ \\
$06 / 06 / 84$ & $<0.0000 \mathrm{E}+00$ & $0.000 \mathrm{~B}+00$ \\
$08 / 11 / 83$ & $0.3870 \mathrm{E}-03$ & $0.293 \mathrm{E}-03$ \\
$08 / 16 / 82$ & $<0.1210 \mathrm{E}-03$ & $0.676 \mathrm{E}-03$
\end{tabular}

\begin{tabular}{|c|c|c|c|}
\hline \multicolumn{2}{|c|}{ Wye Barricade (QC Dupl. } & o-1 in. & $\mathrm{Pu}-238$ \\
\hline $\begin{array}{l}\text { ample } \\
\text { ate }\end{array}$ & $\begin{array}{l}\text { Dup. } \\
\text { No. }\end{array}$ & $\begin{array}{l}\text { Result } \\
\text { pCi/g }\end{array}$ & $\begin{array}{l}+/-2 \\
\text { std. Dev. }\end{array}$ \\
\hline $\begin{array}{l}04 / 29 / 87 \\
04 / 29 / 86 \\
07 / 03 / 85 \\
06 / 06 / 84 \\
08 / 11 / 83 \\
08 / 16 / 82\end{array}$ & -- & $\begin{array}{l}<0.0000 \mathrm{E}+00 \\
<0.3460 \mathrm{E}-04 \\
0.1000 \mathrm{E}-02 \\
0.9390 \mathrm{E}-03 \\
0.6540 \mathrm{E}-03 \\
0.8040 \mathrm{E}-03\end{array}$ & $\begin{array}{l}-.000 E+00 \\
0.0003 E-04 \\
0.693 E-03 \\
0.466 E-03 \\
0.664 E-03 \\
0.335 E-03 \\
0.771 E-03\end{array}$ \\
\hline
\end{tabular}

1 Mile $N$ of mppss No, 2

\begin{tabular}{llll}
6324 & Depth $0-1$ in. & Pu-238 \\
Sample & Dup. Regult & $+/-2$ \\
Date & No. & pCi/g & Std. Dev. \\
\hdashline $01 / 10 / 76$ & & $0.1700 E-02$ & $0.000 E+00$
\end{tabular}

2 Mile $S$ of wPPSS No. 2

6325 Depth $0-1$ in. Pu-238

\begin{tabular}{|c|c|c|c|}
\hline $\begin{array}{l}\text { Sample } \\
\text { Date }\end{array}$ & $\begin{array}{l}\text { Dup. } \\
\text { No. }\end{array}$ & $\begin{array}{l}\text { Result } \\
\text { pCi/g }\end{array}$ & $\begin{array}{l}+/-2 \\
\text { std. Dev. }\end{array}$ \\
\hline $8 / 10 / 76$ & & $0.1390 \mathrm{E}-02$ & $0.000 \mathrm{E}+00$ \\
\hline
\end{tabular}

FFTr Control Plot 62

6015 Depth $0-1$ in, Pu-238

Sample Dup. Result $+/-2$

Date No. pCi/g std. Dev.

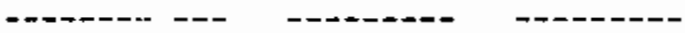

$09 / 24 / 74 \quad 0.3340 E-04 \quad 0.000 E+00$

$10 / 25 / 73 \quad<0.1040 E-02 \quad 0.300 E-02$

$10 / 25 / 72 \quad 0.3700 E-02 \quad 0.000 E+00$

$09 / 30 / 7 \mathrm{~L} \quad 0.1870 \mathrm{E}-02 \quad 0.000 \mathrm{E}+00$ 
TABLEA.4. (contd)

400 Area and Vicinity

Other onsite_focations

$\begin{array}{llll}\text { FFTF Control Plot } 62 & \\ 6038 & \text { Depth } 1-2 \text { in. } & \text { Pu-238 } \\ & & & +/-2 \\ \text { Sample } & \text { Dup. Result } & \text { Std. Dev. } \\ \text { Date } & \text { No. } & \text { pCi/g } & \\ -10 / 25 / 72 & & 0.6050 E-02 & 0.000 \mathrm{E}+00 \\ 09 / 30 / 71 & & 0.0000 \mathrm{E}+00 & 0.000 \mathrm{E}+00\end{array}$

\begin{tabular}{llll}
0.5 Mile NE of FFTF & \\
6282 & Depth $0-1$ in. & Pu-238 \\
Sample & Dup. & Result & $+/-2$ \\
Date & No. & pCi/g & std. Dev. \\
\hline $04 / 29 / 87$ & & $0.2600 \mathrm{E}-03$ & $0.165 \mathrm{E}-03$ \\
$06 / 05 / 85$ & $<0.4050 \mathrm{E}-04$ & $0.744 \mathrm{E}-04$ \\
$05 / 17 / 84$ & $<0.1250 \mathrm{E}-02$ & $0.501 \mathrm{E}-03$ \\
$08 / 12 / 83$ & $<0.0000 \mathrm{E}+00$ & $0.000 \mathrm{E}+00$ \\
$08 / 30 / 82$ & $<-.3760 \mathrm{E}-03$ & $0.751 \mathrm{E}-03$ \\
$08 / 10 / 81$ & $<-.9620 \mathrm{E}-03$ & $0.280 \mathrm{E}-03$ \\
$08 / 05 / 80$ & $<0.2510 \mathrm{E}-03$ & $0.070 \mathrm{E}-03$ \\
$09 / 11 / 79$ & $<-4310 \mathrm{E}-03$ & $0.120 \mathrm{E}-02$ \\
$0 \mathrm{D} / 08 / 78$ & $<0.1370 \mathrm{E}-03$ & $0.340 \mathrm{~B}-02$ \\
$06 / 14 / 77$ & $<-.6460 \mathrm{E}-03$ & $0.670 \mathrm{E}-01$ \\
$08 / 10 / 76$ & $0.1060 \mathrm{E}-02$ & $0.000 \mathrm{E}+00$ \\
$08 / 20 / 75$ & $<0.0000 \mathrm{E}+00$ & $0.000 \mathrm{E}+00$
\end{tabular}

N End of Vernita Bridge

$\mathrm{Pu}-238$

Sample Dup. Result t/- 2

Date No. pCi/g std. Dev.

$05 / 02 / 86$ o.2150E-03 $0.152 \mathrm{E}-03$

$06 / 11 / 85 \quad 0.2200 \mathrm{E}-03 \quad 0.191 \mathrm{E}-03$

$05 / 31 / 84 \quad<0.7450 \mathrm{E}-03 \quad 0.860 \mathrm{E}-03$

$08 / 11 / 83<0.0000 E+00 \quad 0.000 E+00$

$08 / 24 / 82 \quad\langle 0.2680 E-03 \quad 0.892 \mathrm{E}-03$

$09 / 23 / 74 \quad 0.2600 \mathrm{E}-02 \quad 0.000 \mathrm{E}+00$

$10 / 25 / 73 \quad<0.1120 \mathrm{E}-02 \quad 0.300 \mathrm{E}-02$

$10 / 25 / 72 \quad 0.7950 E-02 \quad 0.000 E+00$

$09 / 29 / 71 \quad 0.5000 E-03 \quad 0.000 E+00$

\begin{tabular}{llll} 
N End of Vernita Bridge \\
6028 & Depth $1-2$ in. & Pu-238 \\
& & & \\
Sample & Dup. Result & $+/-2$ \\
Date & No. & pCi/g & Std. Dev. \\
\hline $10 / 25 / 72$ & & $<0.0000 \mathrm{E}+00$ & $0.000 \mathrm{E}+00$ \\
$09 / 29 / 71$ & & $0.1000 \mathrm{E}-02$ & $0.000 \mathrm{E}+00$
\end{tabular}

Hanford Townsite

6017 Depth 0-1 in. Pu-23日

Sample Dup. Regult $\quad+/-2$

Date No. pCi/g std. Dev.

-

$04 / 29 / 87 \quad 0.4950 \mathrm{E}-03 \quad 0.374 E-03$

$06 / 12 / 85 \quad 0.3680 \mathrm{E}-03 \quad 0.231 \mathrm{E}-03$

$05 / 17 / 84<0.0000 E+00 \quad 0.000 E+00$

$08 / 12 / 83 \quad<0.0000 E+00 \quad 0.000 E+00$

$08 / 30 / 82<0.7160 E-04 \quad 0.738 E-03$

$08 / 10 / 81<-.3040 E-03 \quad 0.300 E-03$

$08 / 05 / 80 \quad 0.5600 \mathrm{E}-02 \quad 0.120 \mathrm{E}-02$

$09 / 11 / 79 \quad 0.3460 \mathrm{E}-02 \quad 0.320 \mathrm{E}-02$

$08 / 08 / 7 \theta \quad<-.5240 E-04 \quad 0.140 E-02$

$06 / 14 / 77 \quad<0.9870 E-03 \quad 0.990 E-03$

$08 / 11 / 76 \quad<-.1310 E-04 \quad 0.000 E+00$

$08 / 18 / 75 \quad 0.4720 E-03 \quad 0.000 E+00$

$10 / 25 / 73 \quad<0.2520 E-02 \quad 0.300 E-02$

$10 / 25 / 72 \quad 0.4920 \mathrm{E}-02 \quad 0.000 \mathrm{E}+00$

$09 / 30 / 71 \quad 0.4900 \mathrm{E}-02 \quad 0.000 \mathrm{E}+00$ 
TABLE A.4. (contd)

other onsite Locations

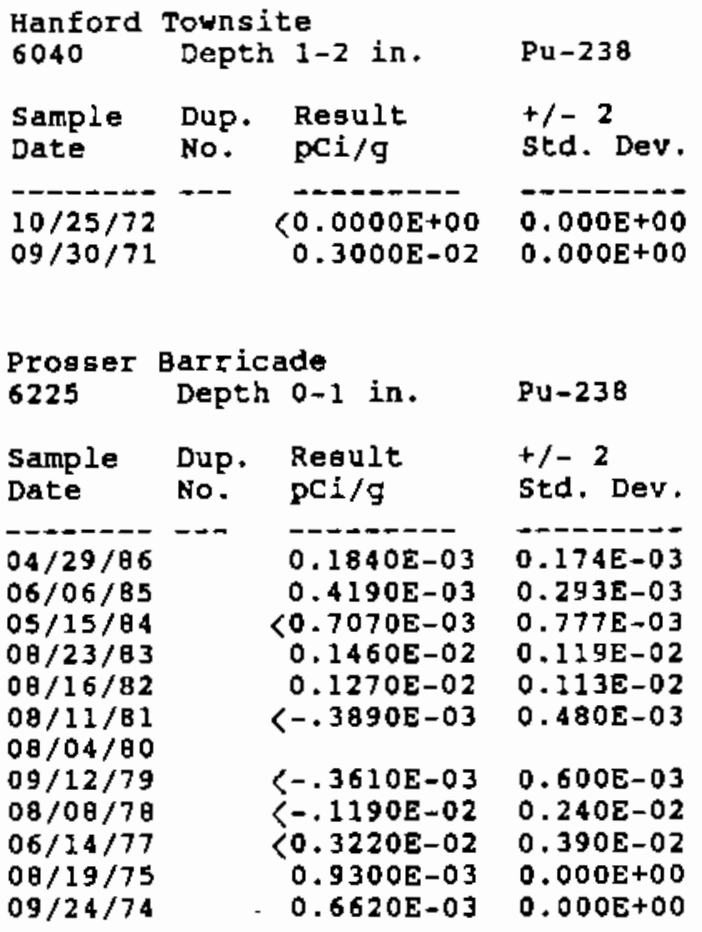

ERC (Emer. Reloc. Center)

\begin{tabular}{llll}
6001 & Depth $0-1$ in. & Pu-238 \\
Sample & Dup. & Regult & $+/-2$ \\
Date & No. & pCi/g & Std. Dev. \\
\hdashline $09 / 24 / 74$ & & $<0.0000 E+00$ & $0.000 E+00$ \\
$10 / 25 / 73$ & & $<0.1630 E-02$ & $0.300 E-02$ \\
$10 / 25 / 72$ & & $0.0000 E+00$ & $0.000 E+00$ \\
$09 / 30 / 71$ & & $<0.0000 \mathrm{E}+00$ & $0.000 \mathrm{E}+00$
\end{tabular}

$\begin{array}{llll}\text { ERC (Emer. Reloc. Center) } & \\ 6024 & \text { Depth } 1-2 \text { in. } & \text { Pu-23日 } \\ & & & \\ \text { Sample } & \text { Dup. } & \text { Regult } & +/-2 \\ \text { Date } & \text { No. } & \text { pCi/g } & \text { Std. Dev. } \\ -10 / 25 / 72 & & 0.1060 E-01 & 0.000 E+00 \\ 09 / 30 / 71 & & 0.3280 E-02 & 0.000 E+00\end{array}$

$E$ of Arid Land Ecol. Lab

6278 Depth $0-1$ in. Pu-238

Sample Dup. Result $+/-2$

Date No. pCi/g std. Dev.

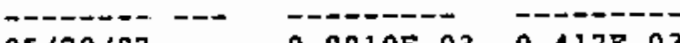

$\begin{array}{lll}05 / 20 / 87 & 0.9910 \mathrm{E}-03 & 0.417 \mathrm{E}-03 \\ 05 / 12 / 85 & 0.8750 \mathrm{E}-03 & 0.342 \mathrm{E}-03\end{array}$

$\begin{array}{lll}06 / 12 / 85 & 0.8750 E-03 & 0.342 E-03 \\ 05 / 24 / 84 & 0.1590 \mathrm{E}-03 & 0.237 \mathrm{E}-03\end{array}$

$\begin{array}{lrl}05 / 24 / 84 & <0.1590 \mathrm{E}-03 & 0.237 \mathrm{E}-03 \\ 08 / 23 / 83 & 0.1620 \mathrm{E}-02 & 0.115 \mathrm{E}-02\end{array}$

$08 / 16 / 82 \quad<0.6080 E-03 \quad 0.620 E-03$

$08 / 13 / 81 \quad 0.9660 \mathrm{E}-03 \quad 0.560 \mathrm{E}-03$

$08 / 05 / 80 \quad 0.3110 \mathrm{E}-02 \quad 0.130 \mathrm{E}-02$

$09 / 12 / 79 \quad 0.1800 \mathrm{E}-02 \quad 0.150 \mathrm{E}-02$

$08 / 10 / 78<-.1010 E-02 \quad 0.190 \mathrm{E}-02$

$06 / 14 / 7\rangle \quad<0.2250 E-03 \quad 0.110 E-02$

$08 / 10 / 76 \quad 0.4860 \mathrm{E}-03 \quad 0.000 E+00$

$08 / 20 / 75 \quad 0.7600 E-04 \quad 0.000 E+00$

Rattlesnake springs

6003 Depth $0-1$ in. Pu-23日

Sample Dup. Result $+/-2$

Date No. pCi/g std. Dev.

05/07/8\% $-0 \quad$ 0.4370E-03

$05 / 23 / 86.0 .1810 \mathrm{E}-03 \quad 0.137 \mathrm{E}-03$

$06 / 06 / 85 \quad 0.6330 E-03 \quad 0.281 E \rightarrow 03$

$05 / 24 / 84 \quad<0.0000 E+00 \quad 0.000 E+00$

$08 / 11 / \mathrm{BJ} \quad<0.0000 \mathrm{E}+00 \quad 0.000 \mathrm{E}+00$

$09 / 02 / 82<0.4670 \mathrm{E}-03 \quad 0.669 \mathrm{E}-03$

$09 / 23 / 74 \quad 0.4660 \mathrm{E}-03 \quad 0.000 \mathrm{E}+00$

$10 / 25 / 73<0.8090 \mathrm{E}-03 \quad 0.300 \mathrm{E}-02$

$10 / 25 / 72 \quad 0.1670 \mathrm{E}-02 \quad 0.000 \mathrm{E}+00$

$09 / 29 / 7 \mathrm{~L} \quad 0.6000 \mathrm{E}-02 \quad 0.000 \mathrm{E}+00$

Rattlesnake Springs

6026 Depth $1-2$ in. Pu-23日

Sample Dup. Result $+/-2$

Date No. $\mathrm{pCi} / \mathrm{g}$ std. Dev.

$\begin{array}{lll}10 / 25 / 72 & 0.1170 \mathrm{E}-02 & 0.000 \mathrm{E}+00\end{array}$

$09 / 29 / 71 \quad<0.0000 \mathrm{E}+00 \quad 0.000 \mathrm{E}+00$ 
TABLE A.4. (contd)

other onsite tocations

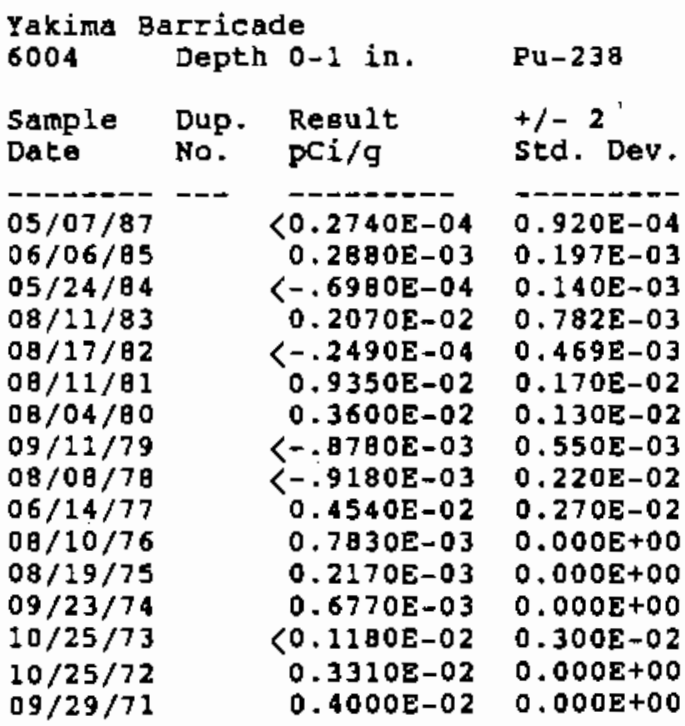

Yakima Barricade

\begin{tabular}{llll}
6027 & Depth $1-2$ in. & Pu-23日 \\
Sample & Dup. & Result & $+/-2$ \\
Date & No. & pCi/g & std. Dev. \\
\hline $10 / 25 / 72$ & & $<0.0000 \mathrm{E}+00$ & $0.000 \mathrm{E}+00$ \\
$09 / 29 / 71$ & & $0.3000 \mathrm{0}-02$ & $0.000 \mathrm{E}+00$
\end{tabular}

A. 31 
TABLE A.5. Plutonium-239,240 Concentrations in Soil at Onsite Locations

200 Areas

NE Corner of $100 \mathrm{H}$ Area

6275 Depth 0-1 in.

\begin{tabular}{llll} 
Sample & Dup. & Result & $+/-2$ \\
Date & No. & pC1/g & Std. Dev. \\
\hline $00 / 20 / 75$ & & $0.3400 \mathrm{E}-01$ & $0.000 \mathrm{E}+00$
\end{tabular}

1 Mile NE of $100 \mathrm{~N}$ Area

0590

Depth 0-1 in.

\begin{tabular}{llll} 
Sarple & Dup. & Result & $+/-2$ \\
Date & No. & pC1/g & std. Dev. \\
\hline $04 / 30 / 86$ & & $0.1450 \mathrm{E}-01$ & $0.136 \mathrm{E}-02$ \\
$06 / 24 / 85$ & & $0.1570 \mathrm{E}-01$ & $0.156 \mathrm{E}-02$ \\
$05 / 17 / 84$ & $0.1460 \mathrm{E}-01$ & $0.184 \mathrm{~B}-02$ \\
$08 / 05 / 83$ & & $0.1160 \mathrm{E}-01$ & $0.252 \mathrm{E}-02$ \\
$08 / 24 / 82$ & & $0.1460 \mathrm{E}-01$ & $0.282 \mathrm{E}-02$
\end{tabular}

1 Mile $E$ of $100 \mathrm{~N}$ Area

0578 Depth $0-1$ in.

\begin{tabular}{llll} 
Sample & Dup. & Regult & $+/-2$ \\
Date & No. & pCi/g & std. Dev. \\
\hline $04 / 29 / 87$ & & $0.2330 \mathrm{E}-01$ & $0.195 \mathrm{E}-02$ \\
$04 / 30 / 86$ & & $0.1160 \mathrm{E}-01$ & $0.140 \mathrm{E}-02$ \\
$06 / 24 / 85$ & & $0.1250 \mathrm{E}-01$ & $0.114 \mathrm{E}-02$ \\
$05 / 17 / 84$ & & $0.1640 \mathrm{E}-01$ & $0.274 \mathrm{E}-02$ \\
$08 / 11 / 83$ & & $0.6900 \mathrm{E}-02$ & $0.142 \mathrm{E}-02$ \\
$08 / 24 / 82$ & & $0.2610 \mathrm{E}-01$ & $0.329 \mathrm{E}-02$
\end{tabular}

1 Mile SE of $100 \mathrm{~N}$ Trench

6320 Depth $0-1$ in.

\begin{tabular}{llll} 
Sample & Dup. & Result & $+/-2$ \\
Date & No. & pC1/g & std. Dev. \\
\hline 0 - $/ 03 / 84$ & & $0.1340 \mathrm{E}-01$ & $0.156 \mathrm{E}-02$ \\
$0 \mathrm{~B} / 11 / 76$ & & $0.4290 \mathrm{E}-02$ & $0.000 \mathrm{E}+00$
\end{tabular}

Wabluke Slope Opposite 100-N

\begin{tabular}{llll}
6006 & Depth $0-1$ in. & Pu-239,240 \\
Sample & Dup. Result & $+/-2$ \\
Date & No. & pCi/g & Std. Dev. \\
\hline $09 / 23 / 74$ & & $0.4120 \mathrm{E}-02$ & $0.000 \mathrm{E}+00$ \\
$10 / 25 / 73$ & & $0.1990 \mathrm{E}-01$ & $0.100 \mathrm{E}-02$ \\
$10 / 25 / 72$ & & $0.1500 \mathrm{E}-01$ & $0.000 \mathrm{E}+00$ \\
$09 / 29 / 71$ & & $0.9000 \mathrm{E}-02$ & $0.000 \mathrm{D}+00$
\end{tabular}

Wahluke Slope Opposite 100-N

6029 Depth $1-21 \mathrm{n}$. Pu-239,240

\begin{tabular}{llll} 
Sample & Dup. & Result & $+/-2$ \\
Date & No. & PCi/g & Std. Dev. \\
\hdashline$-10 / 25 / 72$ & & $0.2200 E-01$ & $0.000 E+00$ \\
$09 / 29 / 71$ & & $0.3000 E-02$ & $0.000 E+00$
\end{tabular}

$\begin{array}{llll}100 \text { Area Fire Station } & \\ 0580 & \text { Depth } 0-1 \text { in. } & \text { Pu-239,240 } \\ & & & \\ \text { Sample } & \text { Dup. Regult } & +/-2 \\ \text { Date } & \text { No. } & \text { pC1/g } & \text { Std. Dev. } \\ & - & - & - \\ 04 / 29 / 87 & & 0.1650 \mathrm{E}-01 & 0.139 \mathrm{E}-02 \\ 04 / 30 / 86 & 0.2960 \mathrm{E}-01 & 0.204 \mathrm{E}-02 \\ 05 / 30 / 85 & 0.2410 \mathrm{E}-01 & 0.160 \mathrm{E}-02 \\ 05 / 17 / 84 & 0.2090 \mathrm{E}-01 & 0.169 \mathrm{E}-02 \\ 08 / 05 / 83 & 0.2220 \mathrm{E}-02 & 0.147 \mathrm{E}-02 \\ 08 / 24 / 82 & 0.1630 \mathrm{E}-01 & 0.285 \mathrm{E}-02\end{array}$

100 F Area Control Plot 58

6018 Depth $0-1$ in. Pu-239,240

\begin{tabular}{llll} 
Sample & Dup. & Regult & $+/-2$ \\
Date & No. & pC $\pm / 9$ & std. Dev. \\
\hline $08 / 11 / 76$ & & $0.7670 \mathrm{E}-02$ & $0.000 \mathrm{E}+00$ \\
$08 / 18 / 75$ & & $0.1570 \mathrm{E}-01$ & $0.000 \mathrm{E}+00$ \\
$09 / 24 / 74$ & & $0.1450 \mathrm{E}-01$ & $0.000 \mathrm{E}+00$ \\
$10 / 25 / 73$ & $0.6370 \mathrm{E}-02$ & $0.100 \mathrm{E}-02$ \\
$10 / 25 / 72$ & & $0.9000 \mathrm{E}-02$ & $0.000 \mathrm{E}+00$ \\
$09 / 30 / 71$ & & $0.1300 \mathrm{E}-01$ & $0.000 \mathrm{E}+00$
\end{tabular}

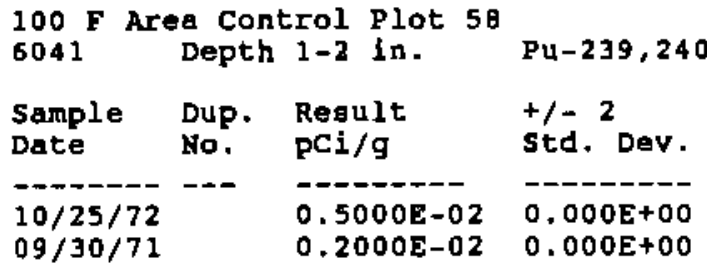


TABLE A. 5 . (contd)

200 East Area

200 E Control Plot 59

6019 Depth $0-1$ in.

Sample Dup. Result $\quad+/-2$

Date No. pCl/g std. Dev.

08/16/75 $-0 \quad \overline{0.2250 E-01}$

$09 / 24 / 74$

$10 / 25 / 72$

$09 / 30 / 71$

$0.5020 \mathrm{E}-01$

$0.1270 \mathrm{E}-01$

$0.9000 E-02$

$0.2000 \mathrm{E}-02$

$0.000 \mathrm{E}+00$

$0.000 \mathrm{E}+00$

$0.100 E-02$

$0.000 \mathrm{E}+00$

$0.000 E+00$

200 E Control Plot 59

6042 Depth 1-2 In.

\begin{tabular}{llll} 
Sample & $\begin{array}{l}\text { Dup. } \\
\text { No. Result }\end{array}$ & $+/-2$ \\
DCis & No. & std. Dev. \\
\hline $10 / 25 / 73$ & & $0.9330 \mathrm{E}-02$ & $0.100 \mathrm{E}-02$ \\
$10 / 25 / 72$ & & $0.9000 \mathrm{E}-02$ & $0.000 \mathrm{E}+00$ \\
$09 / 30 / 71$ & & $0.3000 \mathrm{E}-02$ & $0.000 \mathrm{E}+00$
\end{tabular}

200 E Control plot 59

6188 Depth 2-4 in.

Sample Dup. Regult $+/-2$

Date

pCI/g

$10 / 25 / 73$

$0.4390 \mathrm{E}-02$

std. Dev.

0. $100 \mathrm{0}-\overline{0}$

200 E Control Plot 59

6189 Depth 4-8 in.

\begin{tabular}{|c|c|c|c|}
\hline $\begin{array}{l}\text { Sample } \\
\text { Date }\end{array}$ & $\begin{array}{l}\text { Dup. } \\
\text { No. }\end{array}$ & $\begin{array}{l}\text { Regult } \\
\text { pCi/g }\end{array}$ & $\begin{array}{l}+/-2 \\
\text { std. Dev. }\end{array}$ \\
\hline $10 / 25 / 73$ & & $\langle 0.4800 \mathrm{E}-03$ & $0.100 \mathrm{E}-02$ \\
\hline
\end{tabular}

200 E Control plot 59

6190 Depth 8-12 in. Pu-239,240

Sample Dup, Regult $t /-2$

Date No. pci/g std. Dev.

$10 / 25 / 73=0.1410 \mathrm{E}-0100$
200 ENC (East North Central)

6362 Depth $0-1$ 1n. Pu-239,240

Sample Dup. Regult $\quad+/-2$

Date No. pCl/g std. Dev.

05/07/87 -

04/30/86 $0.1460 E-01 \quad 0.1518-02$

$05 / 31 / 85 \quad 0.2970 \Sigma-01 \quad 0.1898-02$

$05 / 17 / 64 \quad 0.3260 E-01 \quad 0.444 E-02$

$08 / 12 / 83 \quad 0.5110 \mathrm{E}-01 \quad 0.653 \mathrm{E}-02$

$08 / 30 / 82$ 0.5880E-01 $0.858 E-02$

$08 / 10 / 8110.2360 \mathrm{E}-01 \quad 0.220 \mathrm{E}-02$

$08 / 10 / 81 \quad 0.4210 \mathrm{E}-01 \quad 0.240 \mathrm{E}-02$

$08 / 05 / 80 \quad 0.2610 \mathrm{E}-01 \quad 0.190 \mathrm{E}-02$

$09 / 11 / 79 \quad 0.5630 \mathrm{E}-01 \quad 0.550 \mathrm{E}-02$

$08 / 10 / 78 \quad 0.1350 E-01 \quad 0.460 E-02$

$06 / 14 / 77 \quad 0.2450 E-01 \quad 0.500 E-02$

1.25 Mileg Eagt of PUREX

0581 Depth 0-1 in.

Pu-239, 240

Sample Dup. Regult $+/-2$

Date No. pCI/g std. Dev.

-5/07/87 -

04/30/86 $0.1060 \mathrm{E}-01 \quad 0.131 \mathrm{E}-02$

$05 / 31 / 85 \quad 0.2580 \mathrm{E}-01 \quad 0.165 \mathrm{E}-02$

$03 / 15 / 84 \quad 0.1240 \mathrm{E}-01 \quad 0.152 \mathrm{E}-02$

$08 / 05 / 83 \quad 0.1060 \mathrm{E}-01 \quad 0.218 \mathrm{~B}-02$

$08 / 24 / 82 \quad 0.1450 E-01 \quad 0.244 E-02$ 
TABLE A.5. (contd)

200 East Area

200 ESE (East South East)

6022 Depth $0-1$ in.

Pu-239, 240

\begin{tabular}{llll} 
Sample & Dup. & Regult & $+/{ }^{2}$ \\
Date & No. & pCi & std. Dev. \\
\hline $05 / 07 / 87$ & & $0.1180 \mathrm{E}-01$ & $0.120 \mathrm{E}-02$ \\
$04 / 29 / 86$ & & $0.8390 \mathrm{E}-02$ & $0.118 \mathrm{E}-02$ \\
$06 / 12 / 85$ & & $0.2160 \mathrm{E}-01$ & $0.161 \mathrm{E}-02$ \\
$05 / 15 / 84$ & & $0.9130 \mathrm{E}-02$ & $0.168 \mathrm{E}-02$ \\
$08 / 12 / 83$ & & $0.2840 \mathrm{E}-01$ & $0.537 \mathrm{E}-02$ \\
$08 / 30 / 82$ & & $0.2350 \mathrm{E}-01$ & $0.408 \mathrm{E}-02$ \\
$08 / 10 / 81$ & & $0.2630 \mathrm{E}-01$ & $0.400 \mathrm{E}-02$ \\
$08 / 05 / 80$ & & & \\
$09 / 11 / 79$ & & $0.2900 \mathrm{E}-01$ & $0.190 \mathrm{E}-02$ \\
$08 / 10 / 78$ & & $0.1980 \mathrm{E}-01$ & $0.370 \mathrm{E}-02$ \\
$06 / 14 / 77$ & & $0.6180 \mathrm{E}-02$ & $0.230 \mathrm{E}-02$ \\
$08 / 11 / 76$ & & $0.1360 \mathrm{E}-01$ & $0.000 \mathrm{E}+00$ \\
$08 / 18 / 75$ & 5 & $0.1680 \mathrm{E}-01$ & $0.000 \mathrm{E}+00$ \\
$08 / 18 / 75$ & 1 & $0.2210 \mathrm{E}-01$ & $0.000 \mathrm{E}+00$ \\
$08 / 18 / 75$ & 2 & $0.1390 \mathrm{E}-01$ & $0.000 \mathrm{E}+00$ \\
$08 / 18 / 75$ & 4 & $0.1650 \mathrm{E}-01$ & $0.000 \mathrm{E}+00$ \\
$08 / 18 / 75$ & 3 & $0.1290 \mathrm{E}-01$ & $0.000 \mathrm{E}+00$ \\
$09 / 24 / 74$ & & $0.1730 \mathrm{E}-01$ & $0.000 \mathrm{E}+00$ \\
$10 / 25 / 73$ & & $0.3580 \mathrm{E}-01$ & \\
$10 / 25 / 72$ & & $0.2300 \mathrm{E}-01$ & $0.000 \mathrm{E}+00$ \\
$09 / 30 / 71$ & & $0.1700 \mathrm{E}-01$ & $0.000 \mathrm{E}+00$
\end{tabular}

200 ESE (East South Eagt)

6045 Depth 1-2 in.

Pu-239,240

\begin{tabular}{lll} 
Sample & Dup. & Regult \\
Date & No. & pCl/g \\
\hline $10 / 25 / 73$ & & $0.2080 \mathrm{E}-01$ \\
$10 / 25 / 74$ & & $0.8000 \mathrm{E}-02$ \\
$09 / 30 / 71$ & & $0.7000 \mathrm{E}-02$
\end{tabular}

$+/-2$

std. Dev.

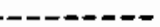

$0.100 E-02$

$0.000 E+00$

$0.000 E+00$

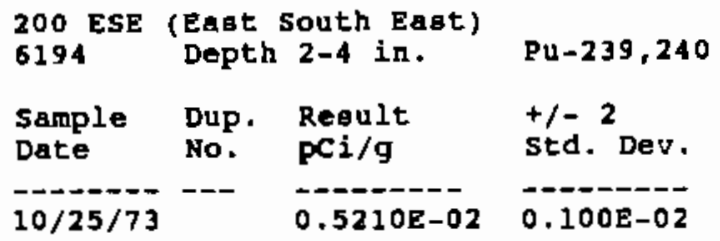

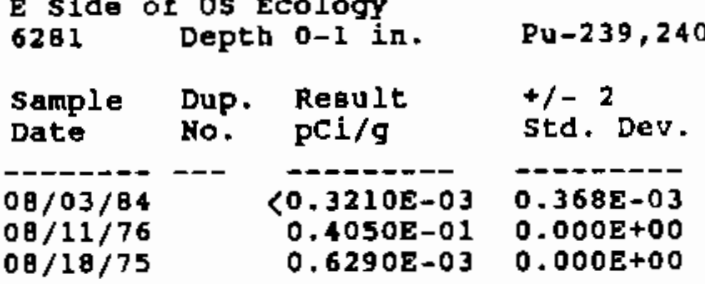

\begin{tabular}{|c|c|c|c|}
\hline $\begin{array}{l}2 \text { Hiles } \\
0582\end{array}$ & $\begin{array}{l}\text { South } \\
\text { Depth }\end{array}$ & $\begin{array}{l}f \text { PUR } \\
0-1 \text { i }\end{array}$ & \\
\hline $\begin{array}{l}\text { Sample } \\
\text { Date }\end{array}$ & $\begin{array}{l}\text { Dup. } \\
\text { No. }\end{array}$ & $\begin{array}{l}\text { Result } \\
\text { pCi/g }\end{array}$ & $\begin{array}{l}+/-2 \\
\text { std. Dev. }\end{array}$ \\
\hline $\begin{array}{l}-05 / 07 / 87 \\
04 / 30 / 86 \\
06 / 24 / 85 \\
05 / 15 / 84 \\
08 / 05 / 83 \\
08 / 24 / 82\end{array}$ & -- & $\begin{array}{l}0.2680 \mathrm{E}-02 \\
0.6700 \mathrm{E}-02 \\
0.4110 \mathrm{E}-02 \\
0.5610 \mathrm{E}-02 \\
0.8 \mathrm{~B} 00 \mathrm{E}-02 \\
0.1150 \mathrm{E}-01\end{array}$ & $\begin{array}{l}0.525 E-03 \\
0.871 E-03 \\
0.145 E-02 \\
0.311 E-02 \\
0.166 E-02 \\
0.137 E-02\end{array}$ \\
\hline
\end{tabular}

3 Miles SSH of PUREX

0583 Depth $0-1$ in. Pu-239, 240

Sample Dup. Regult $+/-2$

Date No. pCi/g std. Dev,

$05 / 07 / 87 \quad 0.6200 \mathrm{E}-03 \quad 0.303 \mathrm{E}-03$

$04 / 30 / 86 \quad 0.4030 E-02 \quad 0.949 E-03$

$06 / 24 / 85 \quad 0.2380 \mathrm{E}-01 \quad 0.219 E-02$

$05 / 15 / 84 \quad 0.3400 E-02 \quad 0.188 E-02$

$08 / 05 / 83 \quad 0.7590 \mathrm{E}-02 \quad 0.120 \mathrm{E}-02$

$00 / 24 / 82 \quad 0.1190 E-01 \quad 0.990 E-03$

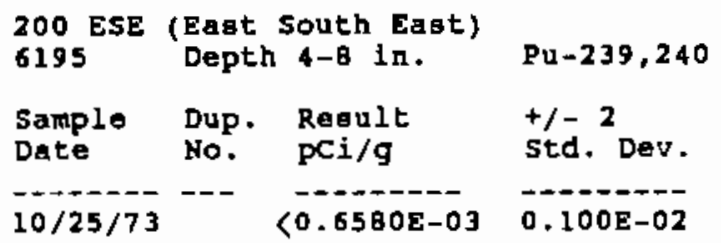


TABLE A.5. (contd)

200 West Area

\begin{tabular}{llll}
$\begin{array}{l}\text { E of } 200 \\
6276\end{array}$ & $\begin{array}{l}\text { West Gate } \\
\text { Depth } 0-1 \text { 1n. }\end{array}$ & Pu-239,240 \\
Sample & Dup. & Regult & $+/-2$ \\
Date & No. & PCi/g & std. Dev. \\
& & - & - \\
\hline $05 / 07 / 87$ & & $0.1700 \mathrm{E}+00$ & $0.430 \mathrm{E}-02$ \\
$05 / 01 / 86$ & $0.3370 \mathrm{E}+00$ & $0.605 \mathrm{E}-02$ \\
$05 / 31 / 85$ & $0.3270 \mathrm{E}+00$ & $0.686 \mathrm{E}-02$ \\
$05 / 15 / 84$ & $0.7370 \mathrm{E}-01$ & $0.415 \mathrm{E}-02$ \\
$08 / 12 / 83$ & $0.8310 \mathrm{E}+00$ & $0.272 \mathrm{E}-01$ \\
$08 / 30 / 82$ & $0.7780 \mathrm{E}+00$ & $0.165 \mathrm{E}-01$ \\
$08 / 10 / 81$ & $0.4200 \mathrm{E}+00$ & $0.750 \mathrm{E}-02$ \\
$08 / 04 / 80$ & $0.6300 \mathrm{E}+00$ & $0.160 \mathrm{E}-01$ \\
$09 / 11 / 79$ & $0.8330 \mathrm{E}+00$ & $0.220 \mathrm{E}-01$ \\
$08 / 09 / 78$ & $0.5610 \mathrm{E}+00$ & $0.200 \mathrm{E}-01$ \\
$06 / 14 / 77$ & $0.1650 \mathrm{E}+00$ & $0.170 \mathrm{E}-01$ \\
$08 / 11 / 76$ & $0.7570 \mathrm{E}-01$ & $0.000 \mathrm{E}+00$ \\
$0 \mathrm{~B} / 1 \mathrm{~B} / 75$ & $0.3060 \mathrm{E}+00$ & $0.000 \mathrm{E}+00$
\end{tabular}

2 Miles $S$ of 200 west

0584 Depth 0-1 in.

\begin{tabular}{llll} 
Sample & Dup. & Result & $+1-2$ \\
Date & No. & pCi/g & std. Dev. \\
\hline $05 / 01 / 86$ & & $0.1250 \mathrm{E}-01$ & $0.120 \mathrm{E}-02$ \\
$05 / 31 / 85$ & & $0.9420 \mathrm{E}-02$ & $0.109 \mathrm{E}-02$ \\
$05 / 17 / 84$ & $0.3620 \mathrm{E}-02$ & $0.187 \mathrm{E}-02$ \\
$08 / 05 / 83$ & $<0.6160 \mathrm{E}-03$ & $0.947 \mathrm{E}-03$ \\
$00 / 24 / 82$ & & $0.3550 \mathrm{E}-02$ & $0.154 \mathrm{E}-02$
\end{tabular}

200 Weat Control Plot 60 6020 Depth $0-1$ in.

$\begin{array}{llll}\text { Sample } & \text { Dup. } & \text { Result } & +/ \overrightarrow{2}^{2} \\ \text { Date } & \text { No. } & \text { pCilg } & \text { std. Dev. } \\ -08 / 18 / 75 & & 0.7660 \mathrm{E}-02 & -0.000 \mathrm{E}+00 \\ 09 / 24 / 74 & & 0.2810 \mathrm{E}-01 & 0.000 \mathrm{E}+00 \\ 10 / 25 / 73 & & 0.4530 \mathrm{E}-01 & 0.100 \mathrm{0}-02 \\ 10 / 25 / 72 & & 0.2100 \mathrm{E}-01 & 0.000 \mathrm{D}+00 \\ 09 / 29 / 71 & & 0.2900 \mathrm{E}-01 & 0.000 \mathrm{E}+00\end{array}$

200 West Control Plot 60

6043 Depth 1-2 in.

$\begin{array}{llll}\text { Sample } & \text { Dup. } & \text { Reeult } & +/{ }^{2} \\ \text { Date } & \text { No. } & \text { pCi/g } & \text { Std. Dev. } \\ -10 / 25 / 73 & & 0.1120 \mathrm{E}-01 & 0.100 \mathrm{E}-02 \\ 10 / 25 / 72 & & 0.9000 \mathrm{E}-02 & 0.000 \mathrm{E}+00 \\ 09 / 30 / 71 & & 0.2000 \mathrm{E}-02 & 0.000 \mathrm{E}+00\end{array}$

\begin{tabular}{|c|c|c|}
\hline $\begin{array}{l}200 \text { west } \\
6191\end{array}$ & Depth 2-4 in. & $\mathrm{Pu}-239,240$ \\
\hline $\begin{array}{l}\text { Sample } \\
\text { Date }\end{array}$ & $\begin{array}{ll}\text { Dup. } & \text { Result } \\
\text { No. } & \text { pCi/g }\end{array}$ & $\begin{array}{l}+/-{ }^{2} \\
\text { std. Dev. }\end{array}$ \\
\hline $10 / 25 / 73$ & $<0.0000 \mathrm{E}+00$ & $0.100 \mathrm{E}-02$ \\
\hline $\begin{array}{l}200 \text { Weat } \\
6192\end{array}$ & $\begin{array}{l}\text { Control Plot } 60 \\
\text { Depth } 4-8 \text { in. }\end{array}$ & $\mathrm{Pu}-239,240$ \\
\hline $\begin{array}{l}\text { Sample } \\
\text { Date }\end{array}$ & $\begin{array}{l}\text { Result } \\
\mathrm{pCi} / \mathrm{g}\end{array}$ & $\begin{array}{l}+/-2 \\
\text { std. Dev. }\end{array}$ \\
\hline $10 / 25 / 73$ & $\langle 0.3120 \mathrm{~B}-03$ & $0.100 \mathrm{E}-02$ \\
\hline
\end{tabular}

200 Weat Control Plot 60 6193 Depth 8-12 in.

Pu-239, 240

Sample Dup. Result

Date No. pC1/g

$+/-2$

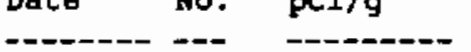

$10 / 25 / 73$

$<0.2400 \mathrm{E}-03$

std. Dev.

$0 . \overline{100 \mathrm{0}-02}$

other 200 Area docations

200 Area Fire station

6021 Depth $0-1$ in.

Pu-239, 240

Sample Dup. Result $+/-2$

Date No. pCi/g Std. Dev.

-

$10 / 25 / 72 \quad 0.1700 \mathrm{E}-01$

$09 / 30 / 71 \quad 0.8000 \mathrm{E}-02$

$0.100 E-02$

$0.000 E+00$

$0.000 \mathrm{E}+00$

200 Area Fire station

6044 Depth 1-2 in. Pu-239,240

Sample Dup. Result $+/-2$

Date No. pCi/g std. Dev.

$10 / 25 / 72=0.1200 \mathrm{E}-01 \quad 0.000 \mathrm{E}+00$

$09 / 30 / 71 \quad 0.3000 \mathrm{E}-02 \quad 0.000 \mathrm{E}+00$ 
TABLE A.5. (contd)

other 200 Area Locations

Gable Pond Control Plot 43

Pu-239, 240

\begin{tabular}{lll} 
Sample & Dup. & Result \\
Date & No. & pCi/g \\
\hline $08 / 11 / 76$ & & $0.7020 \mathrm{E}-02$
\end{tabular}

$+/-2$

std. Dev.

$0.000 E+00$

Inter. $4 \mathrm{~S}$ and Army Loop Rd.

$\begin{array}{llll}6223 & \text { Depth } 0-1 \text { in. } & \text { Pu-239,240 } \\ \text { Sample } & \text { Dup. Result } & +/-2 \\ \text { Date } & \text { No. } & \text { pCi/g } & \text { std. Dev. } \\ -08 / 10 / 76 & & 0.2960 E-02 & 0.000 E+00 \\ 08 / 18 / 75 & & 0.7920 E-02 & 0.000 E+00 \\ 09 / 24 / 74 & & 0.0000 E+00 & 0.000 E+00\end{array}$

Army Loop Rd. Contl. Plot 40

$\begin{array}{llll}6226 & \text { Depth } 0-1 \text { in. } & \text { Pu-239,240 } \\ \text { Sample } & \text { Dup. Regult } & +/-2 \\ \text { Date } & \text { No. } & \text { pCi/g } & \text { Std. Dev. } \\ -09 / 24 / 74 & & 0.4860 \mathrm{E}-02 & 0.000 \mathrm{E}+00\end{array}$

Highway 240 Control Plot 54 6002 Depth 0-1 in.

$\mathrm{Pu}-239,240$

Sample Dup. Regult

Date No. $\mathrm{pCi} / \mathrm{g}$

$+/-2$

-

$09 / 23 / 74$

$10 / 25 / 73$

$10 / 25 / 72$

$09 / 29 / 71$

$0.4120 E-02$

$0.6000 \mathrm{E}-02$

$0.2000 \mathrm{E}-02$

std. Dev.

$----2-1-1$

$0.000 \mathrm{E}+00$

$0.100 E-02$

$0.000 \mathrm{E}+00$

$0.000 \mathrm{E}+00$

Highway 240 Control Plot 54

\begin{tabular}{llll}
6025 & Depth $1-21 \mathrm{n}$. & Pu-239,240 \\
Sample & Dup. & Regult & $+/-2$ \\
Date & No. & pCi/g & std. Dev. \\
\hline $10 / 25 / 72$ & & $0.4000 \mathrm{E}-02$ & $0.000 \mathrm{E}+00$ \\
$09 / 29 / 71$ & & $0.1800 \mathrm{E}-01$ & $0.000 \mathrm{E}+00$
\end{tabular}

300 Area

\begin{tabular}{llll}
$\begin{array}{l}\text { North of } \\
6322\end{array}$ & $\begin{array}{l}300 \text { Area } \\
\text { Depth } 0-1 \text { in. }\end{array}$ & Pu-239,240 \\
Sample & Dup. Result & $+/-2$ \\
Date & No. & pCl/g & std. Dev. \\
\hline $04 / 29 / 87$ & & $0.1430 E-01$ & $0.130 E-02$ \\
$11 / 25 / 86$ & & $0.7580 E-02$ & $0.963 \mathrm{E}-03$ \\
$05 / 31 / 85$ & $0.1010 \mathrm{E}-01$ & $0.108 \mathrm{E}-02$ \\
$05 / 16 / 84$ & $0.6360 \mathrm{E}-02$ & $0.285 \mathrm{E}-02$ \\
$08 / 11 / 83$ & $0.1340 \mathrm{E}-01$ & $0.196 \mathrm{E}-02$ \\
$09 / 03 / 82$ & $0.1580 \mathrm{E}-01$ & $0.312 \mathrm{E}-02$ \\
$08 / 10 / 76$ & & $0.8130 \mathrm{E}-02$ & $0.000 \mathrm{E}+00$
\end{tabular}

Horth Center 300 Area Fence

6279 Depth 0-1 in. Pu-239,240

\begin{tabular}{llll} 
Sample & Dup. & Result & $+/-2$ \\
Date & No. & pCi $/ g$ & Std. Dev. \\
\hdashline $08 / 20 / 75$ & & $0.1060 \mathrm{E}-01$ & $0.000 E+00$
\end{tabular}

\begin{tabular}{|c|c|c|c|}
\hline $\begin{array}{l}300 \text { Area } \\
6222\end{array}$ & $\begin{array}{l}\text { South } \\
\text { Depth }\end{array}$ & $\begin{array}{l}\text { Gate } \\
0-1 \text { in. }\end{array}$ & Pu $-239,240$ \\
\hline $\begin{array}{l}\text { Sample } \\
\text { Date }\end{array}$ & $\begin{array}{l}\text { Dup. } \\
\text { No. }\end{array}$ & $\begin{array}{l}\text { Result } \\
\text { PCi/g }\end{array}$ & $\begin{array}{l}+/-2 \\
\text { std. Dev. }\end{array}$ \\
\hline $09 / 24 / 74$ & $\cdots$ & $0.2190 \mathrm{E}-01$ & $0.000 \mathrm{E}+0 \overline{0}$ \\
\hline
\end{tabular}

South of 300 Area

6323 Depth $0-1$ in. Pu-239, 240

Sample Dup. Result $+/-2$

Date Ho. pCi/g std. Dev.

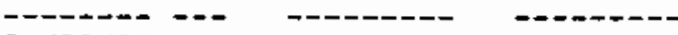

$04 / 29 / 86 \quad 0.1460 \mathrm{E}-01 \quad 0.130 \mathrm{E}-02$

$05 / 31 / 85 \quad 0.1770 E-010.149 E-02$

$05 / 16 / 84 \quad 0.2150 E-01 \quad 0.232 E-02$

$08 / 11 / 83 \quad 0.2160 \mathrm{E}-01 \quad 0.130 \mathrm{E}-02$

$09 / 03 / 82 \quad 0.1920 \mathrm{E}-01 \quad 0.281 \mathrm{E}-02$

$08 / 10 / 76 \quad 0.9420 \mathrm{E}-02 \quad 0.000 \mathrm{E}+00$ 
TABLEA.5. (contd)

300_Area

331 Bldg, Control plot 50

6014 Depth $0-1$ in.

\begin{tabular}{llll} 
Sarople & Dup. & Result & $+/-2$ \\
Date & No. & PC1/g & std. Dev. \\
\hdashline $08 / 20 / 75$ & & $0.7140 \mathrm{E}-03$ & $0.000 \mathrm{E}+00$ \\
$10 / 25 / 73$ & & $<0.7300 \mathrm{E}-03$ & $0.100 \mathrm{E}-02$ \\
$10 / 25 / 72$ & $0.3000 \mathrm{E}-02$ & $0.000 \mathrm{E}+00$ \\
$09 / 30 / 71$ & & $0.1400 \mathrm{E}-01$ & $0.000 \mathrm{E}+00$
\end{tabular}

331 Bldg, Control Plot 50 6037 Depth 1-2 in.

Pu-239, 240

\begin{tabular}{llll} 
Sample & Dup. & Regu1t & $+/-2$ \\
Date & No. & pCi/g & Std. Dev. \\
\hline $10 / 25 / 72$ & & $0.3000 \mathrm{E}-02$ & $0.000 \mathrm{E}+00$ \\
$09 / 30 / 71$ & & $0.5000 \mathrm{E}-02$ & $0.000 \mathrm{E}+00$
\end{tabular}

400 Area and Vicinity

\begin{tabular}{|c|c|c|c|}
\hline 6016 & Dept & $0-1$ in. & $\mathrm{Pu}-239,240$ \\
\hline $\begin{array}{l}\text { Samp1e } \\
\text { Date }\end{array}$ & $\begin{array}{l}\text { Dup. } \\
\text { No. }\end{array}$ & $\begin{array}{l}\text { Result } \\
\text { pci/g }\end{array}$ & $\begin{array}{l}+/-2 \\
\text { std. Dev. }\end{array}$ \\
\hline $\begin{array}{l}-04 / 29 / 87 \\
04 / 29 / 87 \\
07 / 03 / 85 \\
05 / 15 / 84 \\
08 / 11 / 83 \\
08 / 16 / 82 \\
08 / 10 / 81 \\
08 / 05 / 80 \\
09 / 11 / 79 \\
08 / 08 / 78 \\
06 / 14 / 77 \\
10 / 25 / 73 \\
10 / 25 / 72 \\
09 / 30 / 71\end{array}$ & 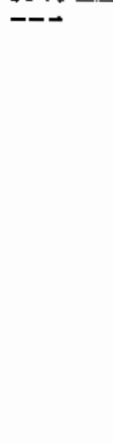 & $\begin{array}{l}0.1070 \mathrm{E}-01 \\
0.1730 \mathrm{E}-01 \\
0.1380 \mathrm{E}-01 \\
0.1670 \mathrm{E}-01 \\
0.1800 \mathrm{E}-01 \\
0.1160 \mathrm{E}-01 \\
0.1300 \mathrm{E}-01 \\
0.1780 \mathrm{E}-01 \\
0.6310 \mathrm{E}-02 \\
0.9480 \mathrm{E}-02 \\
0.1160 \mathrm{E}-01 \\
0.1400 \mathrm{E}-01 \\
0.1400 \mathrm{E}-01\end{array}$ & $\begin{array}{l}0.103 \mathrm{E}-02 \\
0.149 \mathrm{E}-02 \\
0.233 \mathrm{E}-02 \\
0.216 \mathrm{E}-02 \\
0.270 \mathrm{E}-02 \\
0.130 \mathrm{E}-02 \\
0.230 \mathrm{E}-02 \\
0.290 \mathrm{E}-02 \\
0.210 \mathrm{E}-02 \\
0.490 \mathrm{E}-02 \\
0.000 \mathrm{E}+00 \\
0.000 \mathrm{E}+00 \\
0.000 \mathrm{E}+00\end{array}$ \\
\hline
\end{tabular}

Wye Barricade

$\begin{array}{llll}6039 & \text { Depth } 1-2 \text { in. } & \text { Pu-239,240 } \\ \text { Sample } & \text { Dup. Reoult } & +/-2 \\ \text { Date } & \text { No. } & \text { peilg } & \text { Std. Dev. } \\ -10 / 25 / 72 & & 0.1600 \mathrm{E}-01 & 0.000 \mathrm{E}+00 \\ 09 / 30 / 71 & & 0.9000 \mathrm{E}-02 & 0.000 \mathrm{E}+00\end{array}$

\begin{tabular}{llll}
$\begin{array}{l}\text { Wye Barricade (OC Dupl. A) } \\
\text { O816 } \\
\text { Depth } 0-1 \text { in. }\end{array}$ & Pu-239,240 \\
Sample & Dup. Regult & $+/-2$ \\
Date & No. & PCi/g & std. Dev. \\
\hline $04 / 29 / 87$ & & $0.6350 E-02$ & $0.952 E-03$ \\
$04 / 29 / 86$ & $0.1680 \mathrm{E}-02$ & $0.602 \mathrm{E}-03$ \\
$07 / 03 / 85$ & $0.7930 \mathrm{E}-02$ & $0.113 \mathrm{E}-02$ \\
$06 / 06 / 84$ & $0.4870 \mathrm{E}-02$ & $0.325 \mathrm{E}-02$ \\
$08 / 11 / 83$ & $0.1050 \mathrm{E}-01$ & $0.152 \mathrm{E}-02$ \\
$08 / 16 / 82$ & $0.1960 \mathrm{E}-01$ & $0.278 \mathrm{E}-02$
\end{tabular}

Tye Barricade (OC Dupl. B) 0817 Depth 0-1 in.

Pu-239, 240

\begin{tabular}{llll}
$\begin{array}{l}\text { Sample } \\
\text { Date }\end{array}$ & $\begin{array}{l}\text { Dup. } \\
\text { No. }\end{array}$ & $\begin{array}{l}\text { Result } \\
\text { pCi/g }\end{array}$ & $\begin{array}{l}+/-2 \\
\text { Std. Dev. }\end{array}$ \\
\hline $04 / 29 / 87$ & & $0.8230 \mathrm{E}-02$ & $0.993 \mathrm{E}-03$ \\
$04 / 29 / 86$ & & $0.1730 \mathrm{E}-02$ & $0.489 \mathrm{E}-03$ \\
$07 / 03 / 85$ & & $0.2070 \mathrm{E}-01$ & $0.217 \mathrm{E}-02$ \\
$06 / 06 / 84$ & & $0.5980 \mathrm{E}-02$ & $0.168 \mathrm{E}-02$ \\
$08 / 11 / 83$ & & $0.1710 \mathrm{E}-01$ & $0.163 \mathrm{E}-02$ \\
$08 / 16 / 82$ & & $0.1690 \mathrm{E}-01$ & $0.226 \mathrm{E}-02$
\end{tabular}

\begin{tabular}{|c|c|c|c|}
\hline $\begin{array}{l}1 \text { M110 } N \\
6324\end{array}$ & $\begin{array}{l}\text { of } \mathrm{Wr} \\
\text { Depth }\end{array}$ & $\begin{array}{l}\text { Pss No. } 2 \\
0-1 \text { in. }\end{array}$ & Pu-239, 240 \\
\hline $\begin{array}{l}\text { Sample } \\
\text { Date }\end{array}$ & $\begin{array}{l}\text { Dup. } \\
\text { No. }\end{array}$ & $\begin{array}{l}\text { Regult } \\
\text { pCilg }\end{array}$ & $\begin{array}{l}+/-{ }^{2} \\
\text { std. Dev. }\end{array}$ \\
\hline $08 / 10 / 76$ & -- & $0.8190 \mathrm{E}-03$ & $0.000 \mathrm{E}+00$ \\
\hline
\end{tabular}

1 Mile $s$ of WPPS No. 2

6325 Depth $0-1$ in. Pu-239,240

Sample Dup. Result t/- 2

Date No. pCi/g std. Dev.

$\overline{08 / 10 / 76} \quad \overline{0.1330 \mathrm{E}-01} \quad \overline{0.000 \mathrm{E}+00}$ 


\section{TABLE A.5. (contd)}

400 Area and Vicinity

FFTF Control Plot 62

6015 Depth $0-1$ in.

Pu-239, 240

SE Side of FFTF

\begin{tabular}{llll} 
Sample & Dup. & Reeult & $+/-2$ \\
Date & No. & pCi/g & std. Dev. \\
\hline $09 / 24 / 74$ & & $0.2820 \mathrm{E}-02$ & $0.000 \mathrm{E}+00$ \\
$10 / 25 / 73$ & & $0.3490 \mathrm{E}-02$ & $0.100 \mathrm{E}-02$ \\
$10 / 25 / 72$ & & $0.5000 \mathrm{E}-02$ & $0.000 \mathrm{E}+00$ \\
$09 / 30 / 71$ & & $0.4000 \mathrm{E}-02$ & $0.000 \mathrm{E}+00$
\end{tabular}

FFTF Control Plot 62

6038 Depth 1-2 in.

Pu-239, 240

\begin{tabular}{llll} 
Sample & Dup. & Regult & $+/-2$ \\
Date & No. & pCi/g & Std. Dev. \\
\hline $10 / 25 / 72$ & & $0.1100 \mathrm{E}-01$ & $0.000 \mathrm{E}+00$ \\
$09 / 30 / 71$ & & $<0.0000 \mathrm{E}+00$ & $0.000 \mathrm{0}+00$
\end{tabular}

0.5 HIle NE of FFTP

\begin{tabular}{llll}
6282 & Depth & $0-11 \mathrm{n}$, & Pu-239,240 \\
Sample & Dup. & Regult & $+/-2$ \\
Date & No. & pCilg & std. Dev. \\
\hline $04 / 29 / 87$ & & $0.5540 \mathrm{E}-02$ & $0.760 \mathrm{E}-03$ \\
$06 / 05 / 85$ & $0.2500 \mathrm{E}-02$ & $0.503 \mathrm{E}-03$ \\
$05 / 17 / 84$ & $<0.2100 \mathrm{E}-02$ & $0.669 \mathrm{E}-03$ \\
$08 / 12 / 83$ & $0.2870 \mathrm{E}-02$ & $0.667 \mathrm{E}-03$ \\
$08 / 30 / 82$ & $0.2410 \mathrm{E}-02$ & $0.130 \mathrm{E}-02$ \\
$08 / 10 / 81$ & $0.2610 \mathrm{E}-02$ & $0.790 \mathrm{E}-03$ \\
$08 / 05 / 80$ & $0.3880 \mathrm{E}-02$ & $0.160 \mathrm{E}-02$ \\
$09 / 11 / 79$ & $0.2410 \mathrm{E}-02$ & $0.230 \mathrm{E}-02$ \\
$08 / 08 / 78$ & $<0.1700 \mathrm{E}-02$ & $0.360 \mathrm{E}-02$ \\
$06 / 14 / 77$ & $0.1650 \mathrm{E}+00$ & $0.120 \mathrm{E}+00$ \\
$08 / 10 / 76$ & $0.1340 \mathrm{E}-01$ & $0.000 \mathrm{E}+00$ \\
$08 / 20 / 75$ & $0.9250 \mathrm{E}-03$ & $0.000 \mathrm{E}+00$
\end{tabular}

\begin{tabular}{|c|c|c|c|}
\hline $\begin{array}{l}\text { N End of } \\
6005\end{array}$ & $\begin{array}{l}\text { Vern } \\
\text { Dept }\end{array}$ & $\begin{array}{l}\text { Bridge } \\
0-1 \text { in. }\end{array}$ & Pu-299, 24 \\
\hline $\begin{array}{l}\text { Sample } \\
\text { Date }\end{array}$ & $\begin{array}{l}\text { Dup. } \\
\text { No. }\end{array}$ & $\begin{array}{l}\text { Regult } \\
\mathrm{pci} / \mathrm{g}\end{array}$ & $\begin{array}{l}+/-2 \\
\text { std. Dev. }\end{array}$ \\
\hline $\begin{array}{l}-05 / 02 / 86 \\
05 / 11 / 85 \\
05 / 31 / 84 \\
08 / 11 / 83 \\
08 / 24 / 82 \\
09 / 23 / 74 \\
10 / 23 / 73 \\
10 / 25 / 72 \\
09 / 29 / 71\end{array}$ & -- & $\begin{array}{l}0.3030 \mathrm{E}-02 \\
0.9460 \mathrm{E}-02 \\
0.5950 \mathrm{E}-02 \\
0.1500 \mathrm{E}-01 \\
0.8910 \mathrm{E}-02 \\
0.4440 \mathrm{E}-02 \\
0.1270 \mathrm{E}-01 \\
0.3000 \mathrm{E}-02 \\
0.7000 \mathrm{E}-02\end{array}$ & $\begin{array}{l}0.576 \mathrm{E}-03 \\
0.105 \mathrm{E}-02 \\
0.243 \mathrm{E}-02 \\
0.261 \mathrm{E}-02 \\
0.234 \mathrm{E}-02 \\
0.000 \mathrm{E}+00 \\
0.000 \mathrm{E}+00 \\
0.000 \mathrm{E}+00 \\
0.000 \mathrm{E}+00\end{array}$ \\
\hline $\begin{array}{l}\text { N End of } \\
6028\end{array}$ & $\begin{array}{l}\text { Verni } \\
\text { Depth }\end{array}$ & $\begin{array}{l}\text { ta Bridge } \\
\text { 1-2 in. }\end{array}$ & $P u-239,240$ \\
\hline $\begin{array}{l}\text { Sample } \\
\text { Date }\end{array}$ & $\begin{array}{l}\text { Dup. } \\
\text { No. }\end{array}$ & $\begin{array}{l}\text { Result } \\
\mathrm{pCi} / \mathrm{g}\end{array}$ & $\begin{array}{l}+/-2 \\
\text { std. Dev. }\end{array}$ \\
\hline - & --- & $\begin{array}{l}0.6000 \mathrm{E}-03 \\
0.5000 \mathrm{E}-02\end{array}$ & $\begin{array}{l}0.000 \mathrm{E}+00 \\
0.000 \mathrm{E}+00\end{array}$ \\
\hline
\end{tabular}


TABLE A. 5. (contd)

Other Onsite Locations

Hanford Townite

\begin{tabular}{llll}
6017 & Depth $0-1$ in. & Pu-239,240 \\
Sample & Dup. & Result & $+/-2$ \\
Date & No. & pCi/g & std. Dev. \\
\hline $04 / 29 / 87$ & & $0.1920 \mathrm{E}-01$ & $0.233 \mathrm{E}-02$ \\
$06 / 12 / 85$ & & $0.5850 \mathrm{E}-02$ & $0.880 \mathrm{E}-03$ \\
$05 / 17 / 84$ & $0.1560 \mathrm{E}-01$ & $0.270 \mathrm{E}-02$ \\
$08 / 12 / 83$ & $0.2110 \mathrm{E}-01$ & $0.366 \mathrm{E}-02$ \\
$08 / 30 / 82$ & $0.1530 \mathrm{E}-01$ & $0.277 \mathrm{E}-02$ \\
$08 / 10 / 81$ & $0.2310 \mathrm{E}-01$ & $0.160 \mathrm{E}-02$ \\
$08 / 05 / 80$ & $0.4190 \mathrm{E}-01$ & $0.290 \mathrm{E}-02$ \\
$09 / 11 / 79$ & $0.1980 \mathrm{E}-01$ & $0.670 \mathrm{E}-02$ \\
$08 / 08 / 78$ & $<-1720 \mathrm{E}-03$ & $0.110 \mathrm{E}-02$ \\
$06 / 14 / 77$ & $0.1880 \mathrm{E}-01$ & $0.290 \mathrm{E}-02$ \\
$08 / 11 / 76$ & $0.1090 \mathrm{E}-01$ & $0.000 \mathrm{E}+00$ \\
$08 / 18 / 75$ & $0.1960 \mathrm{E}-01$ & $0.000 \mathrm{E}+00$ \\
$10 / 25 / 73$ & $0.1500 \mathrm{E}-01$ & $0.000 \mathrm{E}+00$ \\
$10 / 25 / 72$ & $0.2400 \mathrm{E}-01$ & $0.000 \mathrm{E}+00$ \\
$09 / 30 / 71$ & $0.2200 \mathrm{E}-01$ & $0.000 \mathrm{E}+00$
\end{tabular}

Hanford Towneite

\begin{tabular}{llll}
6040 & Depth $1-2$ in. & Pu-239,240 \\
Sample & Dup. Result & $+/-2$ \\
Date & No. & pCi/g & std. Dev. \\
\hline $10 / 25 / 72$ & & $0.4000 \mathrm{E}-02$ & $0.000 \mathrm{E}+00$ \\
$09 / 30 / 71$ & & $<0.0000 \mathrm{E}+00$ & $0.000 \mathrm{E}+00$
\end{tabular}

Progger Barricade

\begin{tabular}{llll}
6225 & Depth $0-1$ 1n. & Pu-239,240 \\
Sample & Dup. & Regult & $+/-2$ \\
Date & No. & pCi/g & std. Dev. \\
\hline $04 / 29 / 86$ & & $0.7920 E-02$ & $0.987 E-03$ \\
$06 / 06 / 85$ & $0.1920 E-01$ & $0.1108 E-02$ \\
$05 / 15 / 84$ & $0.3870 E-02$ & $0.161 E-02$ \\
$08 / 23 / 83$ & $0.2030 E-01$ & $0.443 E-02$ \\
$08 / 16 / 82$ & $0.3290 E-01$ & $0.428 E-02$ \\
$08 / 11 / 81$ & $0.5650 E-02$ & $0.120 E-02$ \\
$08 / 04 / 80$ & & & \\
$09 / 12 / 79$ & $0.1600 E-01$ & $0.310 E-02$ \\
$08 / 08 / 78$ & $0.1140 E-01$ & $0.520 E-02$ \\
$06 / 14 / 77$ & $0.1370 E-01$ & $0.610 E-02$ \\
$08 / 19 / 75$ & $0.4480 E-02$ & $0.000 E+00$ \\
$09 / 24 / 74$ & $0.1010 \mathrm{E}-01$ & $0.000 \mathrm{E}+00$
\end{tabular}

\begin{tabular}{|c|c|c|c|}
\hline $\begin{array}{l}\text { ERC (Emer } \\
6001\end{array}$ & $\begin{array}{l}\text { Rel } \\
\text { Depth }\end{array}$ & $\begin{array}{l}\text { c. Center) } \\
0-1 \text { in. }\end{array}$ & $P u-239,240$ \\
\hline $\begin{array}{l}\text { Sample } \\
\text { Date }\end{array}$ & $\begin{array}{l}\text { Dup. } \\
\text { No. }\end{array}$ & $\begin{array}{l}\text { Reeult } \\
\text { pci/g }\end{array}$ & $\begin{array}{l}+/-2 \\
\text { std. Dev. }\end{array}$ \\
\hline $\begin{array}{l}-09 / 24 / 74 \\
10 / 25 / 73 \\
10 / 25 / 72 \\
09 / 30 / 71\end{array}$ & --- & $\begin{array}{l}0.2540 \mathrm{E}-02 \\
0.1850 \mathrm{E}-01 \\
0.9000 \mathrm{E}-02 \\
0.1500 \mathrm{E}-01\end{array}$ & $\begin{array}{l}0.000 \mathrm{E}+00 \\
0.000 \mathrm{E}+00 \\
0.000 \mathrm{E}+00 \\
0.000 \mathrm{E}+00\end{array}$ \\
\hline $\begin{array}{l}\text { ERC (Emer } \\
6024\end{array}$ & Fepth & $\begin{array}{l}\text { ac. Center) } \\
1-2 \text { In. }\end{array}$ & $\mathrm{Pu}-239,240$ \\
\hline $\begin{array}{l}\text { Sample } \\
\text { Date }\end{array}$ & $\begin{array}{l}\text { Dup. } \\
\text { No. }\end{array}$ & $\begin{array}{l}\text { Result } \\
\text { pCi/g }\end{array}$ & $\begin{array}{l}+/-2 \\
\text { std. Dev. }\end{array}$ \\
\hline $\begin{array}{l}10 / 25 / 72 \\
09 / 30 / 71\end{array}$ & --- & $\begin{array}{l}0.1300 \mathrm{E}-01 \\
0.5000 \mathrm{E}-02\end{array}$ & $\begin{array}{l}0.000 \mathrm{E}+00 \\
0.000 \mathrm{E}+00\end{array}$ \\
\hline
\end{tabular}

E of Arld Land Ecol. Lab 6278 Depth $0-1 \mathrm{in}$.

Pu $-239,240$

Sample Dup. Regult $+/-2$

Date No, pC1/g std. Dev.

$05 / 20 / 87-0.2430 \mathrm{E}-01$

$06 / 12 / 85 \quad 0.3420 \mathrm{E}-01$

$05 / 24 / 84 \quad 0.9100 E-02$

$00 / 23 / 83 \quad 0.3110 \mathrm{E}-01$

$0 B / 16 / 82 \quad 0.2950 E-01$

$08 / 13 / 81$

$0.10108-01$

$0.2300 \mathrm{E}-01$

$0.2800 \mathrm{E}-01$

0.5620 E-02

$0.1670 E-01$

$0.5840 E-02$

$0.1310 \mathrm{E}-01$

$0.201 E-02$

$0.210 E-02$

$0.139 \mathrm{E}-02$

$0.502 \mathrm{E}-02$

$0.242 \mathrm{E}-02$

$0.120 \mathrm{E}-02$

$0.310 \mathrm{E}-02$

$0.480 \mathrm{E}-02$

$0.360 \mathrm{E}-02$

$0.3508-02$

$0.000 E+00$

$0.000 E+00$ 
TABLE A.5. (contd)

other onsite Locations

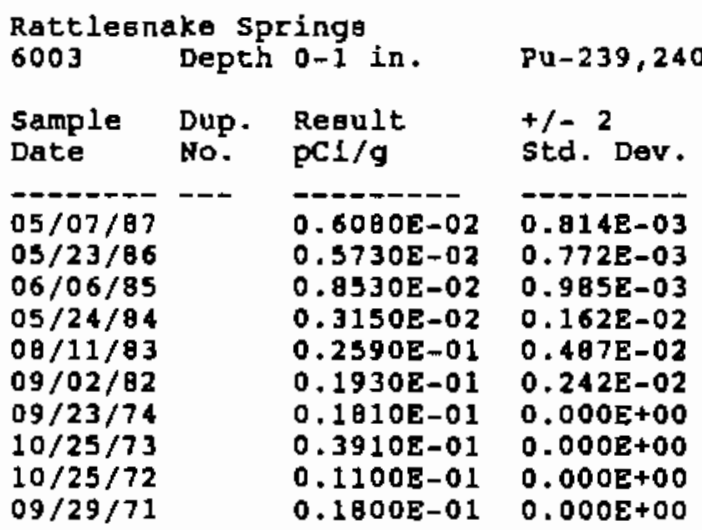

Yaxima Barricade

6027 Depth 1-2 in. Pu-239,240

Sample Dup. Result $+/-2$

Date No. pCi/g Std. Dev.

$\begin{array}{lll}10 / 25 / 72 & 0.5000 \mathrm{E}-02 & 0.000 \mathrm{E}+00\end{array}$

$09 / 29 / 71 \quad 0.3000 \mathrm{E}-02 \quad 0.000 \mathrm{E}+00$

\begin{tabular}{llll}
\multicolumn{2}{l}{$\begin{array}{l}\text { Rattlesnake Springs } \\
6026\end{array}$} & Depth $1-2$ in. & Pu-239,240 \\
& & & \\
Sample & Dup. Result & $+/-2$ \\
Date & No. & pCi/g & std. Dev. \\
$-10 / 25 / 72$ & -- & $0.6000 \mathrm{E}-02$ & $0.000 \mathrm{E}+00$ \\
$09 / 29 / 71$ & & $0.4000 \mathrm{E}-03$ & $0.000 \mathrm{E}+00$
\end{tabular}

\begin{tabular}{|c|c|c|c|}
\hline $\begin{array}{l}\text { Yaxima Bt } \\
6004\end{array}$ & $\begin{array}{l}\text { irric } \\
\text { Dept }\end{array}$ & ${ }_{0-1}^{e}$ in. & Pu-239,240 \\
\hline $\begin{array}{l}\text { Sarple } \\
\text { Date }\end{array}$ & $\begin{array}{l}\text { Dup. } \\
\text { No. }\end{array}$ & $\begin{array}{l}\text { Regult } \\
\text { pCi/g }\end{array}$ & $\begin{array}{l}+/=2 \\
\text { std. Dev. }\end{array}$ \\
\hline $\begin{array}{l}-\overline{05 / 07 / 87} \\
06 / 06 / 85 \\
05 / 24 / 84 \\
08 / 11 / 83 \\
00 / 17 / 82 \\
00 / 11 / 81 \\
08 / 04 / 80 \\
09 / 11 / 79 \\
00 / 08 / 78 \\
06 / 14 / 77 \\
08 / 10 / 76 \\
00 / 19 / 75 \\
09 / 23 / 74 \\
10 / 25 / 73\end{array}$ & -- & $\begin{array}{l}-.-15 \\
0.1510 \mathrm{E}-02 \\
0.2150 \mathrm{E}-01 \\
0.1570 \mathrm{E}-02 \\
0.1470 \mathrm{E}-01 \\
0.1060 \mathrm{E}-01 \\
0.1770 \mathrm{E}-01 \\
0.1700 \mathrm{E}-01 \\
0.4500 \mathrm{E}-02 \\
0.1960 \mathrm{E}-01 \\
0.8270 \mathrm{E}-02 \\
0.5720 \mathrm{E}-02 \\
0.4800 \mathrm{E}-02 \\
0.3440 \mathrm{E}-02 \\
0.1220 \mathrm{E}-01\end{array}$ & $\begin{array}{l}-0.404 \mathrm{E}-03 \\
0.154 \mathrm{E}-02 \\
0.105 \mathrm{E}-02 \\
0.204 \mathrm{E}-02 \\
0.148 \mathrm{E}-02 \\
0.220 \mathrm{E}-02 \\
0.260 \mathrm{E}-02 \\
0.200 \mathrm{E}-02 \\
0.660 \mathrm{E}-02 \\
0.330 \mathrm{E}-02 \\
0.000 \mathrm{E}+00 \\
0.000 \mathrm{E}+00 \\
0.000 \mathrm{E}+00 \\
0.000 \mathrm{E}+00\end{array}$ \\
\hline $\begin{array}{l}10 / 25 / 72 \\
09 / 29 / 71\end{array}$ & & $\begin{array}{l}0.9000 \mathrm{E}-02 \\
0.5000 \mathrm{E}-02\end{array}$ & $\begin{array}{l}0.000 \mathrm{E}+00 \\
0.000 \mathrm{E}+00\end{array}$ \\
\hline
\end{tabular}


TABLE A.6. Americium-24I Concentrations in Soil at onsite Locations

200 Eastmarea

200 EKC (East North Ceritral)

6362 Depth 0-1 in. Ara-24i

$\begin{array}{llll}\begin{array}{l}\text { Sample } \\ \text { Date }\end{array} & \begin{array}{l}\text { Dup. } \\ \text { No. }\end{array} & \begin{array}{l}\text { Result } \\ \text { pCi/g }\end{array} & \begin{array}{l}+/ 2 \\ \text { Std. Dev. }\end{array} \\ -05 / 31 / 85 & & 0.5960 E-02 & 0.397 E-02 \\ 05 / 17 / 84 & & <0.7780 \mathrm{E}-02 & 0.768 E-02 \\ 08 / 12 / 83 & & -.2040 \mathrm{E}-02 & 0.217 \mathrm{E}-02 \\ 08 / 30 / 82 & <0.2610 \mathrm{E}-01 & 0.335 \mathrm{E}-01 \\ 06 / 14 / 77 & & 0.8530 \mathrm{E}-01 & 0.670 \mathrm{E}-01\end{array}$

1.25 Hiles Eagt of PUREX

0581 Depth $0-1$ in. Am-241

\begin{tabular}{llll} 
Sample & Dup. & Result & $+/-2$ \\
Date & No. & DCilg & Std. Dev. \\
\hdashline $05 / 31 / 85$ & & $--3020 E-04$ & $0.249 E-02$ \\
$05 / 15 / 84$ & $0.2610 E-02$ & $0.249 E-02$ \\
$08 / 05 / 83$ & $<0.2010 E-02$ & $0.370 E-02$ \\
$08 / 24 / 82$ & & $-0.1020 E-02$ & $0.325 E-01$
\end{tabular}

200 ESE (East South Eagt)

6022 Depth $0-1$ in.

$A \sqrt{-241}$

sample

Bup. Result

Date No. $\mathrm{PCi} / \mathrm{g}$

$05 / 07 / 87<0.1920 \mathrm{E}-01$

$04 / 29 / 86 \quad<0.5550 \mathrm{E}-02$

$06 / 12 / 85 \quad 0.8000 E-02$

$05 / 15 / 84$

$08 / 12 / 83$

$08 / 30 / 82$

$06 / 14 / 77$

$<0.3070 \mathrm{E}-02$

$<-.1400 \mathrm{E}-02$

$0.5920 \mathrm{E}-01$

$<0.5940 \mathrm{E}-01$

$+/-2$

std. Dev.

$0.223 E-01$

$0.780 \mathrm{E}-02$

$0.581 \mathrm{E}-02$

$0.560 \mathrm{E}-02$

$0.347 \mathrm{E}-02$

$0.256 \mathrm{E}-01$

$0.670 \mathrm{E}-01$

2 Miles South of PUREX

0582 Depth $0-1$ in.

\begin{tabular}{|c|c|c|c|}
\hline $\begin{array}{l}\text { Sample } \\
\text { Date }\end{array}$ & $\begin{array}{l}\text { Dup. } \\
\text { No. }\end{array}$ & $\begin{array}{l}\text { Result } \\
\mathrm{pCi} / \mathrm{g}\end{array}$ & $\begin{array}{l}+/-{ }^{2} \\
\text { std. Dev. }\end{array}$ \\
\hline $\begin{array}{l}-1-1 / 85 \\
06 / 24 / 85 \\
05 / 15 / 84 \\
08 / 05 / 83 \\
08 / 24 / 82\end{array}$ & --- & $\begin{array}{l}<0.7830 \mathrm{E}-03 \\
<-.1160 \mathrm{E}-02 \\
\langle 0.4430 \mathrm{O}-02 \\
<-.6900 \mathrm{0}-02\end{array}$ & $\begin{array}{l}0.361 \mathrm{E}-02 \\
0.331 \mathrm{E}-02 \\
0.471 \mathrm{E}-02 \\
0.312 \mathrm{E}-01\end{array}$ \\
\hline
\end{tabular}

3 Miles SSW of PUREX

0583 Depth $0-1$ in. Ain-241

Sample Dup. Result $+/-2$

Date No. pCi/g std. Dev.

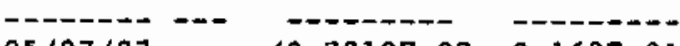

$05 / 07 / 67 \quad<0.7010 E-02 \quad 0.162 \mathrm{E}-01$

$04 / 30 / 86<-.3030 \mathrm{E}-03 \quad 0.574 \mathrm{E}-02$

$06 / 24 / 05 \quad 0.1220 \mathrm{E}-01 \quad 0.654 \mathrm{E}-02$

$05 / 15 / 84<0.2640 E-02 \quad 0.665 E-02$

$08 / 05 / 83<0.1700$ F, $-02 \quad 0.653 E-02$

$00 / 24 / 82<0.1070 \mathrm{E}-01 \quad 0.270 \mathrm{E}-01$

200 west Area

\begin{tabular}{|c|c|c|c|}
\hline $\begin{array}{r}\text { of } 200 \\
276\end{array}$ & $\begin{array}{l}\text { Hest } \\
\text { Eepth }\end{array}$ & $\begin{array}{l}\text { Gate } \\
0-1 \text { in. }\end{array}$ & $A m-241$ \\
\hline $\begin{array}{l}\text { ample } \\
\text { ate }\end{array}$ & $\begin{array}{l}\text { Dup. } \\
\text { No. }\end{array}$ & $\begin{array}{l}\text { Result } \\
\text { pci/y }\end{array}$ & $\begin{array}{l}+/-2 \\
\text { std. Dev. }\end{array}$ \\
\hline $\begin{array}{l}05 / 07 / 37 \\
05 / 6 / 86 \\
05 / 31 / 85 \\
05 / 15 / 84 \\
08 / 12 / 83 \\
08 / 30 / 82 \\
06 / 14 / 77\end{array}$ & $-\cdots$ & $\begin{array}{r}<0.1550 \mathrm{E}-01 \\
0.5280 \mathrm{E}-01 \\
0.2290 \mathrm{E}-01 \\
\angle 0.4040 \mathrm{E}-01 \\
0.69 \mathrm{~B} 0 \mathrm{E}-01 \\
0.5300 \mathrm{E}-01 \\
\langle 0.2170 \mathrm{E}-01\end{array}$ & $\begin{array}{l}0.167 \mathrm{E}-01 \\
0.294 \mathrm{E}-01 \\
0.727 \mathrm{E}-02 \\
0.546 \mathrm{E}-01 \\
0.280 \mathrm{E}-01 \\
0.334 \mathrm{E}-01 \\
0.730 \mathrm{E}-01\end{array}$ \\
\hline
\end{tabular}

2 Mileg 5 of 200 Weat

0584 Depth $0-1$ in.

$A \pi-241$

Sample Dup. Result $+/-2$

Date No. pCi/g std. Dev.

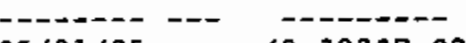

$05 / 31 / 85$

$<0.3220 \mathrm{E}-02$

$05 / 17 / 84$

$<0.4420 \mathrm{E}-02$

$<0.1580 E-02$

$0.636 \mathrm{E}-02$

$\mathrm{nA} / 0 \mathrm{~S} / \mathrm{S3}$

$0.3110 E-01$

$0.515 \mathrm{E}-02$

$0.255 \mathrm{E}-0 \mathrm{I}$ 
TABLEA.6. (contd)

400 Area and Vicinity

\begin{tabular}{|c|c|c|c|}
\hline $\begin{array}{l}\text { Wye Bar } \\
6016\end{array}$ & $\begin{array}{l}\text { 1Cacie } \\
\text { Depth }\end{array}$ & $0-1$ in. & $A m-241$ \\
\hline $\begin{array}{l}\text { Sample } \\
\text { Date }\end{array}$ & $\begin{array}{l}\text { Dup. } \\
\text { No. }\end{array}$ & $\begin{array}{l}\text { Result } \\
\mathrm{pCi} / \mathrm{g}\end{array}$ & $\begin{array}{l}+/-2 \\
\text { std. Dev. }\end{array}$ \\
\hline $\begin{array}{l}-07 / 03 / 85 \\
05 / 15 / 84 \\
08 / 11 / 83 \\
08 / 16 / 82 \\
06 / 14 / 77\end{array}$ & --- & $\begin{array}{r}0.4420 \mathrm{E}-02 \\
<0.1550 \mathrm{E}-02 \\
<0.4110 \mathrm{E}-02 \\
0.3010 \mathrm{E}-01 \\
<0.3840 \mathrm{E}-01\end{array}$ & $\begin{array}{l}0.338 \mathrm{E}-02 \\
0.643 \mathrm{E}-02 \\
0.507 \mathrm{E}-02 \\
0.264 \mathrm{E}-01 \\
0.670 \mathrm{E}-01\end{array}$ \\
\hline
\end{tabular}

\begin{tabular}{|c|c|c|c|}
\hline $\begin{array}{l}\text { SE side } \\
6277\end{array}$ & $\begin{array}{l}\text { of FFTF } \\
\text { Depth }\end{array}$ & $0-1$ in. & $A m-241$ \\
\hline $\begin{array}{l}\text { Sample } \\
\text { pate }\end{array}$ & $\begin{array}{l}\text { Dup. } \\
\text { No. }\end{array}$ & $\begin{array}{l}\text { Regult } \\
\mathrm{pCi} / \mathrm{g}\end{array}$ & $\begin{array}{l}+/-2 \\
\text { std. Dev. }\end{array}$ \\
\hline $06 / 14 / 77$ & --- & $0.6680 \mathrm{E}-01$ & $0.660 \mathrm{E}-01$ \\
\hline
\end{tabular}

Wye Barricade (OC Dupl. A)

\begin{tabular}{llll}
0016 & Depth $0-1$ in. & Am-24I \\
Sample & Dup. & Result & $+/-2$ \\
Date & No. & pCi/g & std. Dev. \\
\hline $04 / 29 / 87$ & & $<0.1620 \mathrm{E}-02$ & $0.405 E-02$ \\
$04 / 29 / 86$ & & $<0.0460 \mathrm{E}-02$ & $0.975 \mathrm{E}-02$ \\
$07 / 03 / 85$ & & $0.6000 \mathrm{E}-02$ & $0.510 \mathrm{E}-02$ \\
$06 / 06 / 84$ & & $0.5850 \mathrm{E}-02$ & $0.989 \mathrm{E}-03$ \\
$08 / 11 / 83$ & $<0.1600 \mathrm{E}-02$ & $0.425 \mathrm{E}-02$ \\
$08 / 16 / 82$ & & $<-.1930 \mathrm{E}-01$ & $0.303 \mathrm{E}-01$
\end{tabular}

Hanford Townsite
6017 Depth 0-1 in. An-241

Sample Dup. Result $+/-2$

Date No. pCi/g std. Dev.

$06 / 12 / 85-\quad<0.2030 \mathrm{E}-02 \quad 0.495 \mathrm{E}-02$

$05 / 17 / 84 \quad<0.1810 \mathrm{E}-02 \quad 0.369 \mathrm{E}-02$

$08 / 12 / 83 \quad 0.1360 \mathrm{E}-01 \quad 0.950 \mathrm{E}-02$

$08 / 30 / 82<-.3260 E-01 \quad 0.371 E-01$

$\begin{array}{llll}06 / 14 / 77 & 0.3640 E-01 & 0.000 E+00\end{array}$

Wye Barricade (OC Dupl. B)

\begin{tabular}{|c|c|c|c|}
\hline 817 & Depth & $0-1$ in. & $A m-241$ \\
\hline $\begin{array}{l}\text { ample } \\
\text { ate }\end{array}$ & $\begin{array}{l}\text { Dup. } \\
\text { No. }\end{array}$ & $\begin{array}{l}\text { Result } \\
\text { pCl/g }\end{array}$ & $\begin{array}{l}+/-2 \\
\text { std. Dev. }\end{array}$ \\
\hline $\begin{array}{l}-0----- \\
04 / 29 / 87 \\
04 / 29 / 86 \\
07 / 03 / 85 \\
06 / 06 / 84 \\
08 / 11 / 83 \\
08 / 16 / 82\end{array}$ & --- & $\begin{array}{r}<0.3400 \mathrm{E}-03 \\
0.9870 \mathrm{E}-02 \\
0.5760 \mathrm{E}-02 \\
<0.1560 \mathrm{E}-02 \\
0.9520 \mathrm{E}-02 \\
<0.6800 \mathrm{E}-02\end{array}$ & $\begin{array}{l}0.568 \mathrm{E}-02 \\
0.02 \\
0.890 \mathrm{E}-02 \\
0.332 \mathrm{E}-02 \\
0.287 \mathrm{E}-02 \\
0.820 \mathrm{E}-02 \\
0.343 \mathrm{E}-02\end{array}$ \\
\hline
\end{tabular}

\begin{tabular}{|c|c|c|c|}
\hline $\begin{array}{l}0.5 \mathrm{Mile} \\
6282\end{array}$ & $\begin{array}{l}\text { NE of } \\
\text { Depth }\end{array}$ & $\begin{array}{l}\text { FFTE } \\
0-1 \text { in. }\end{array}$ & Am-241 \\
\hline $\begin{array}{l}\text { Sample } \\
\text { Date }\end{array}$ & $\begin{array}{l}\text { Dup. } \\
\text { No. }\end{array}$ & $\begin{array}{l}\text { Result } \\
\mathrm{pci} / \mathrm{g}\end{array}$ & $\begin{array}{l}+/-2 \\
\text { std. Dev. }\end{array}$ \\
\hline $06 / 14 / 77$ & $=-$ & $<0.5460 \mathrm{E}-01$ & $0.670 \mathrm{E}-01$ \\
\hline
\end{tabular}

$E$ of Arid Land Ecol. Lab

6278 Depth 0-1 in. Am-241

Sample Dup. Result $t /-2$

Date No. pCi/g std. Dev.

$06 / 14 / 77 \quad<0.2830 \mathrm{E}-01 \quad 0.660 \mathrm{E}-01$ 
TABLE A.6. (contd)

Qther onsite Locations

Rattlesnake Springs

6003 Depth 0-1 in. Am-241

Sample Dup. Result $+/-2$

Date No. pCl/g Std. Dev.

$06 / 06 / 85$ 0.1720E-01 $0.111 \mathrm{E}-01$

$05 / 24 / 84<0.1490 E-02 \quad 0.254 E-02$

$0 \mathrm{~B} / 11 / 83 \quad 0.4280 \mathrm{E}-02 \quad 0.389 \mathrm{E}-0$ ?

$09 / 02 / 82<-.4660 \mathrm{E}-01 \quad 0.330 \mathrm{E}-\mathrm{C} 1$

Yakina Barricade

6004 Depth $0-1$ in. Am-241

Sample Dup. Regult $+/-2$

Date No. $\mathrm{pCi} / \mathrm{g}$ std. Dev.

$06 / 14 / 77^{-0} \quad\langle 0.2350 \mathrm{E}-01 \quad 0.660 \mathrm{E}-01$ 
TABLE A.7. Uranium Concentrations in Soil at Onsite Locations

100 Areas

\begin{tabular}{|c|c|c|c|}
\hline E Corner & r of 1 & 00 N Area & Urantum \\
\hline $\begin{array}{l}\text { Sample } \\
\text { Date }\end{array}$ & $\begin{array}{l}\text { Dup. } \\
\text { No. }\end{array}$ & $\begin{array}{l}\text { Regult } \\
\mathrm{pCi} / \mathrm{g}\end{array}$ & $\begin{array}{l}+/-2 \\
\text { std. Dev. }\end{array}$ \\
\hline $8 / 20 / 75$ & -- & $0.2420 \mathrm{E}+00$ & $0.000 \mathrm{E}+00$ \\
\hline $\begin{array}{c}\text { Mile NE } \\
590\end{array}$ & E of 10 & $\begin{array}{c}00 \text { N Area } \\
0-1 \text { in. }\end{array}$ & Uranium \\
\hline $\begin{array}{l}\text { Sample } \\
\text { Date }\end{array}$ & $\begin{array}{l}\text { Dup. } \\
\text { No. }\end{array}$ & $\begin{array}{l}\text { Regult } \\
\text { pCi/g }\end{array}$ & $\begin{array}{l}+/-2 \\
\text { std. Dev. }\end{array}$ \\
\hline $\begin{array}{l}-4-1 / 30 / 86 \\
04 / 24 / 85 \\
06 / 2417 / 84 \\
05 / 17 / 05 / 83 \\
08 / 05 / 24 / 82\end{array}$ & & $\begin{array}{l}0.1910 \mathrm{E}+00 \\
0.4870 \mathrm{E}+00 \\
0.4160 \mathrm{E}+00 \\
0.3900 \mathrm{E}+00 \\
0.2300 \mathrm{E}+00\end{array}$ & $\begin{array}{l}0.000 \mathrm{E}+00 \\
0.162 \mathrm{E}+00 \\
0.113 \mathrm{E}+00 \\
0.109 \mathrm{E}+00 \\
0.804 \mathrm{E}-01\end{array}$ \\
\hline
\end{tabular}

1 Mile $\mathrm{E}$ of $100 \mathrm{~N}$ Area 0578 Depth 0-1 in.

\begin{tabular}{llll} 
Sample & Dup. & Regult & $+/-2$ \\
Date & No. & pCi/g & std. Dev. \\
\hline $04 / 29 / 87$ & & $0.3440 E+00$ & $0.000 E+00$ \\
$04 / 30 / 86$ & & $0.4500 E+00$ & $0.000 E+00$ \\
$06 / 24 / 85$ & & $0.3980 E+00$ & $0.131 E+00$ \\
$05 / 17 / 84$ & & $0.3170 E+00$ & $0.878 E-01$ \\
$08 / 11 / 83$ & $0.2750 E+00$ & $0.766 E-01$ \\
$08 / 24 / 82$ & & $0.2200 E+00$ & $0.770 E-01$
\end{tabular}

1 Mile SE of $100 \mathrm{~N}$ Trench 6320 Depth 0-1 in.

\begin{tabular}{llll} 
Sample & Dup. & Result & $+/-2$ \\
Date & No. & gCi/g & std. Dev. \\
\hline $08 / 03 / 94$ & & $0.2760 \mathrm{E}+00$ & $0.000 \mathrm{E}+00$ \\
$08 / 11 / 76$ & & $0.1550 \mathrm{D}+00$ & $0.000 \mathrm{E}+00$
\end{tabular}

Wahluke slope Opposite 100-N

$\begin{array}{llll}6006 & \text { Depth } 0-1 \text { in. } & \text { Uranium } \\ \text { Sample } & \text { Dup. } & \text { Result } & +/-2 \\ \text { Date } & \text { No. } & \text { pCi/g } & \text { Std. Dev. } \\ -09 / 23 / 74 & & 0.2360 \mathrm{E}+00 & 0.000 \mathrm{E}+00 \\ 10 / 25 / 73 & & 0.6970 \mathrm{E}+00 & 0.000 E+00\end{array}$

\begin{tabular}{llll}
100 Area & Fire Station & \\
0580 & Depth $0-1$ in. & Uranium \\
& & & \\
Sample & Dup. Result & $+/-2$ \\
Date & No. & pCi/g & std. Dev. \\
\hline $04 / 29 / 87$ & & $0.3500 E+00$ & $0.000 E+00$ \\
$04 / 30 / 86$ & $0.5290 E+00$ & $0.000 E+00$ \\
$05 / 30 / 85$ & $0.4370 E+00$ & $0.145 E+00$ \\
$05 / 17 / 84$ & $0.4460 E+00$ & $0.122 E+00$ \\
$08 / 05 / 83$ & $0.2150 E+00$ & $0.606 E-01$ \\
$08 / 24 / 82$ & & $0.3220 E+00$ & $0.113 E+00$
\end{tabular}

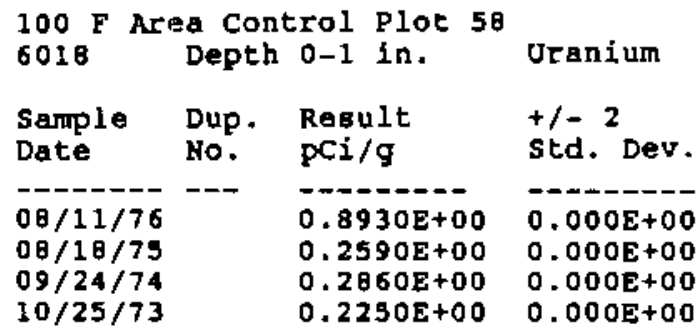

200 sast Area

$\begin{array}{llll}200 \text { E Control Plot } 59 & \\ 6019 & \text { Depth } 0-1 \text { in. } & \text { Uranium } \\ & & & \\ \text { Sample } & \text { Dup. } & \text { Regult } & +/-2 \\ \text { Date } & \text { No. } & \text { pCi/g } & \text { Std. Dev. } \\ -09 / 18 / 75 & & 0.2990 E+00 & 0.000 E+00 \\ 09 / 24 / 74 & 0.1310 E+00 & 0.000 E+00 \\ 10 / 25 / 73 & & 0.5120 E+00 & 0.000 E+00\end{array}$

\begin{tabular}{llll}
200 E Control Plot 59 & \\
6042 & Depth $1-2$ in. & Uranium \\
& & & \\
Sample & Dup. Result & $+/-2$ \\
Date & No. & pCi/g & Std. Dev. \\
\hdashline $10 / 25 / 73$ & & $0.1500 \mathrm{E}+00$ & $0.000 \mathrm{D}+00$
\end{tabular}


TABLEA.7. (contd)

200 East Area

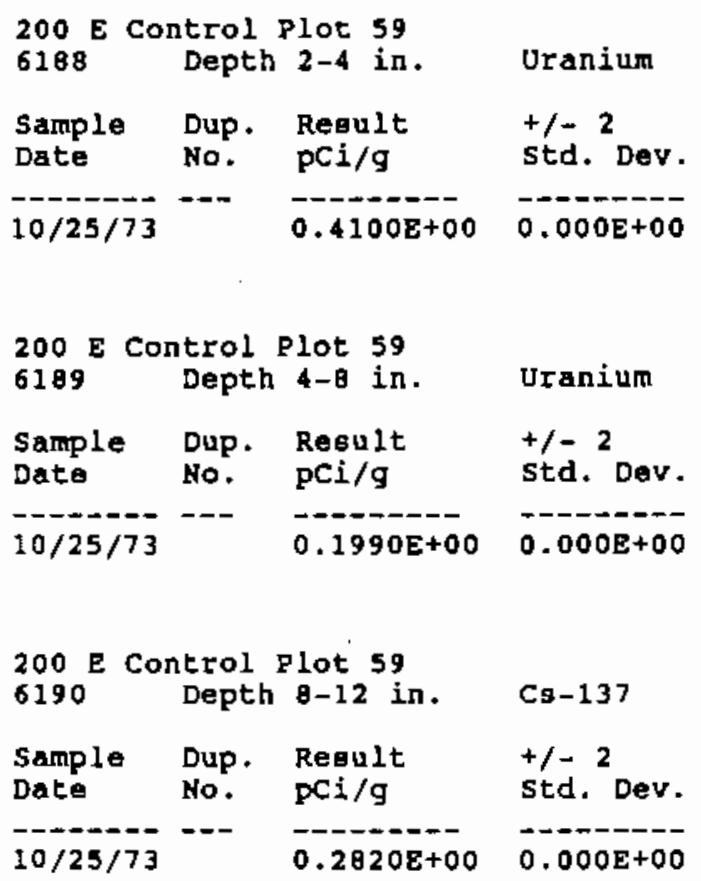

200 ENC (East North Central)

$\begin{array}{llll}6362 & \text { Depth } 0-1 \text { in. } & \text { Uranium } \\ \text { Sarnple } & \text { Dup. } & \text { Result } & +/-2 \\ \text { Date } & \text { No. } & \text { pCi } / g & \text { std. Dev. } \\ & & & \\ 05 / 07 / 87 & & 0.2320 E+00 & 0.000 E+00 \\ 04 / 30 / 86 & & 0.2830 E+00 & 0.000 E+00 \\ 05 / 31 / 85 & & 0.3850 E+00 & 0.126 E+00 \\ 05 / 17 / 84 & & 0.3620 E+00 & 0.982 E-01 \\ 08 / 12 / 83 & & 0.2530 E+00 & 0.710 E-01 \\ 08 / 30 / 82 & & 0.4460 E+00 & 0.157 E+00 \\ 08 / 10 / 81 & 1 & 0.3990 E+00 & 0.140 E+00 \\ 08 / 10 / 81 & & 0.6560 E+00 & 0.230 E+00 \\ 08 / 05 / 80 & & 0.2740 E+00 & 0.960 E-01 \\ 09 / 11 / 79 & & 0.3600 E+00 & 0.130 E+00 \\ 08 / 10 / 78 & & 0.5460 E+00 & 0.150 E+00 \\ 06 / 14 / 77 & & 0.2640 E+00 & 0.680 E-01\end{array}$

1.25 Miles East of PUREX 0581 Depth $0-1$ in.

\begin{tabular}{|c|c|c|c|}
\hline $\begin{array}{l}\text { ample } \\
\text { ate }\end{array}$ & $\begin{array}{l}\text { Dup. } \\
\text { No. }\end{array}$ & $\begin{array}{l}\text { Regult } \\
\text { pCi/g }\end{array}$ & $\begin{array}{l}+/-2 \\
\text { std. Dev. }\end{array}$ \\
\hline $\begin{array}{l}-05 / 07 / 87 \\
04 / 30 / 86 \\
05 / 31 / 85 \\
05 / 15 / 84 \\
08 / 05 / 83\end{array}$ & -- & $\begin{array}{l}0.3120 \mathrm{E}+00 \\
0.4720 \mathrm{D}+00 \\
0.4640 \mathrm{0}+00 \\
0.3200 \mathrm{0}+00 \\
0.2590 \mathrm{E}+00 \\
0.3190 \mathrm{0}+00\end{array}$ & $\begin{array}{l}0.000 \mathrm{E}+00 \\
0.000 \mathrm{E}+00 \\
0.151 \mathrm{E}+00 \\
0.020 \mathrm{E}-01 \\
0.724 \mathrm{E}-01 \\
0.112 \mathrm{E}+00\end{array}$ \\
\hline
\end{tabular}

200 ESE (Eagt South East) 6022 Depth 0-1 in.

Uranium

\begin{tabular}{|c|c|c|c|}
\hline $\begin{array}{l}\text { Sample } \\
\text { Date }\end{array}$ & $\begin{array}{l}\text { Dup. } \\
\text { No. }\end{array}$ & $\begin{array}{l}\text { Regult } \\
\text { pCl/g }\end{array}$ & $\begin{array}{l}+/-^{2} \\
\text { std. Dev. }\end{array}$ \\
\hline $\begin{array}{l}-1---\bar{l} \\
05 / 07 / 87 \\
04 / 29 / 86 \\
06 / 12 / 85 \\
05 / 15 / 84 \\
08 / 12 / 83 \\
08 / 30 / 82 \\
08 / 10 / 81 \\
08 / 05 / 80 \\
09 / 11 / 79 \\
08 / 10 / 78 \\
06 / 14 / 77 \\
08 / 11 / 76 \\
0 \theta / 18 / 75 \\
08 / 18 / 75 \\
08 / 18 / 75 \\
08 / 18 / 75 \\
00 / 18 / 75 \\
09 / 24 / 74 \\
10 / 25 / 73\end{array}$ & $\begin{array}{l}5 \\
4 \\
1 \\
2 \\
3\end{array}$ & 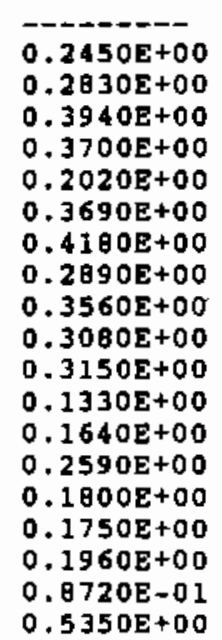 & $\begin{array}{l}0.000 \mathrm{E}+00 \\
0.000 \mathrm{E}+00 \\
0.129 \mathrm{E}+00 \\
0.700 \mathrm{E}-01 \\
0.570 \mathrm{E}-01 \\
0.129 \mathrm{E}+00 \\
0.150 \mathrm{E}+00 \\
0.100 \mathrm{E}+00 \\
0.120 \mathrm{E}+00 \\
0.150 \mathrm{E}+00 \\
0.600 \mathrm{E}-01 \\
0.000 \mathrm{E}+00 \\
0.000 \mathrm{E}+00 \\
0.000 \mathrm{0}+00 \\
0.000 \mathrm{E}+00 \\
0.000 \mathrm{E}+00 \\
0.000 \mathrm{E}+00 \\
0.000 \mathrm{0}+00 \\
0.000 \mathrm{0}+00\end{array}$ \\
\hline $\begin{array}{l}200 \text { ESE } \\
6045\end{array}$ & $\begin{array}{l}\text { (East } \\
\text { Depth }\end{array}$ & $\begin{array}{l}\text { South East) } \\
\text { 1-2 in. }\end{array}$ & Uranium \\
\hline $\begin{array}{l}\text { Sample } \\
\text { Date }\end{array}$ & $\begin{array}{l}\text { Dup. } \\
\text { No. }\end{array}$ & $\begin{array}{l}\text { Result } \\
\mathrm{pCi} / \mathrm{g}\end{array}$ & $\begin{array}{l}+/-2 \\
\text { std. Dev. }\end{array}$ \\
\hline$\overline{10 / 25 / 73}$ & --- & $0.20608+00$ & $0.000 E+00$ \\
\hline
\end{tabular}

\begin{tabular}{|c|c|c|c|}
\hline 6194 & Dept? & $2-4$ in. & uranium \\
\hline $\begin{array}{l}\text { Sample } \\
\text { Date }\end{array}$ & $\begin{array}{l}\text { Dup. } \\
\text { No. }\end{array}$ & $\begin{array}{l}\text { Result } \\
\mathrm{pCi} / \mathrm{g}\end{array}$ & $\begin{array}{l}+/-2 \\
\text { std. Dev. }\end{array}$ \\
\hline $0 / 25 / 73$ & --- & $0.2880 \mathrm{E}+00$ & $0.000 \mathrm{E}+00$ \\
\hline
\end{tabular}


TABLE A.7. (contd)

200 East Area

200 ESE (Eagt South Eagt)

6195 Depth 4-8 in.

\begin{tabular}{lll} 
Sample & Dup. & Regult \\
Date & No. & pCi/g \\
\hline $\mathbf{1 0 / 2 5 / 7 3}$ & & $0.2400 \mathrm{E}+00$
\end{tabular}

Uranium

$+/-2$

std. Dev.

$0.000 \mathrm{E}+00$

200 ESE (East South Eagt)

6196 Depth $8-12$ in.

\begin{tabular}{lll} 
Sample & Dup. & Result \\
Date & No. & pcl/g \\
\hline $10 / 25 / 73$ & & $0.26908+00$
\end{tabular}

Urantum

$+/-2$

std, Dev.

$0.000 E+00$

2 Mileg South of PUREX

0582 Depth 0-1 in.

Sample Dup. Result

Date No. pCi/g

$05 / 07 / 87-0.2170 \mathrm{E}+00$

$04 / 30 / 86$

$06 / 24 / 85$

$05 / 15 / 84$

$08 / 05 / 83$

$08 / 24 / 82$

$0.3230 \mathrm{E}+00$

$0.3350 \mathrm{E}+00$

$0.4600 \mathrm{E}+00$

$0.1770 \mathrm{E}+00$

$0.3040 \mathrm{E}+00$

3 Miles SSW of PURgX

0583 Depth 0-1 in.

Sample Dup. Reault $+/-2$

Date No. pCi/g std. Dev.

$\overrightarrow{05 / 07 / 87}-\overline{0.18908+00} \quad \overrightarrow{0.000 \mathrm{E}+00}$

$04 / 30 / 86 \quad 0.3240 E+00$

$06 / 24 / 85 \quad 0.3270 E+00$

$05 / 15 / 84 \quad 0.1030 E+01$

$08 / 05 / 83 \quad 0.2790 E+00$

$08 / 24 / 82 \quad 0.2700 E+00$

E side of Us Ecology

$$
6281 \text { Depth 0-1 in. }
$$

\begin{tabular}{llll} 
Sample & Dup. & Regult & $+/ \mathbf{2}^{2}$ \\
Dato & No. & pCi/g & std. Dev. \\
\hdashline $08 / 03 / 84$ & & $0.4540 E+00$ & $0.000 E+00$ \\
$08 / 11 / 76$ & & $0.1870 E+00$ & $0.000 E+00$ \\
$08 / 18 / 75$ & & $0.2070 E+00$ & $0.000 E+00$
\end{tabular}

200 West Area

\begin{tabular}{|c|c|c|c|}
\hline 276 & $\begin{array}{l}\text { Went } \\
\text { Depth }\end{array}$ & $\begin{array}{l}\text { Gate } \\
0-1 \text { in. } \\
\text { Reault } \\
\text { pcisg }\end{array}$ & $\begin{array}{l}\text { Uranium } \\
+/ \vec{~}^{2} \\
\text { std. Dev. }\end{array}$ \\
\hline $\begin{array}{l}--1-7 \bar{l} \\
5 / 07 / 87 \\
5 / 01 / 86 \\
5 / 31 / 85 \\
5 / 15 / 84 \\
8 / 12 / 83 \\
\theta / 30 / 82 \\
\theta / 10 / 81 \\
\theta / 04 / 80 \\
9 / 11 / 79 \\
8 / 09 / 78 \\
6 / 14 / 77 \\
\theta / 11 / 76 \\
8 / 18 / 75\end{array}$ & --- & $\begin{array}{l}-.-1 \\
0.3890 \mathrm{E}+00 \\
0.4760 \mathrm{E}+00 \\
0.4280 \mathrm{0}+00 \\
0.5300 \mathrm{E}+00 \\
0.5300 \mathrm{E}+00 \\
0.7310 \mathrm{E}+00 \\
0.7180 \mathrm{E}+00 \\
0.6190 \mathrm{E}+00 \\
0.6050 \mathrm{E}+00 \\
0.7030 \mathrm{E}+00 \\
0.3310 \mathrm{E}+00 \\
0.2390 \mathrm{E}+00 \\
0.2070 \mathrm{E}+00\end{array}$ & $\begin{array}{l}-.000 \mathrm{E}+00 \\
0.0000 \mathrm{E}+00 \\
0.000 \\
0.140 \mathrm{E}+00 \\
0.292 \mathrm{E}+00 \\
0.145 \mathrm{E}+00 \\
0.256 \mathrm{E}+00 \\
0.250 \mathrm{E}+00 \\
0.220 \mathrm{E}+00 \\
0.210 \mathrm{E}+00 \\
0.150 \mathrm{E}+00 \\
0.680 \mathrm{E}-01 \\
0.000 \mathrm{E}+00 \\
0.000 \mathrm{E}+00\end{array}$ \\
\hline
\end{tabular}

200 West Control plot 60

6020 Depth 0-1 in.

Sample Dup. Result

Date No. pCi/g

$0.000 E+00$

$0.112 \mathrm{E}+00$

$0.146 E+00$

$0.776 \mathrm{E}-01$

$0.943 \mathrm{E}-01$

$08 / 18 / 75$

$08 / 16 / 75$

$10 / 25 / 73$

$0.27805+00$

$0.2780 \mathrm{E}+00$

$0.7150 \mathrm{E}+00$

Uranium

$+1-2$

std. Dev.

$0.0008+00$

$0.000 \mathrm{E}+00$

$0.000 \mathrm{E}+00$
200 weet Control Plot 60 6043 Depth 1-2 in.

Sample Dup, Result Date No. pCi/g

$10 / 25 / 73-0.3470 \mathrm{E}+00$
Vranium

$+1-2$ std. Dev.

$0.0008+00$ 
TABLE A.7. (contd)

200 West Area

\begin{tabular}{|c|c|c|c|}
\hline $\begin{array}{l}200 \text { ฟевt } \\
6191\end{array}$ & Dept & 2-4 in. & Uranium \\
\hline $\begin{array}{l}\text { Sample } \\
\text { Date }\end{array}$ & $\begin{array}{l}\text { Dup. } \\
\text { No. }\end{array}$ & $\begin{array}{l}\text { Result } \\
\mathrm{pCi} / \mathrm{g}\end{array}$ & $\begin{array}{l}+/{ }^{2} 2 \\
\text { std. Dev. }\end{array}$ \\
\hline $10 / 25 / 73$ & & $0.3910 \mathrm{E}+00$ & $0.000 \mathrm{E}+00$ \\
\hline
\end{tabular}

200 West Control plot 60

\begin{tabular}{llll}
6192 & Depth $4-8$ in. & Uramium \\
Sample & Dup. Regult & $+/-2$ \\
Date & No. & pCi/g & std. Dev. \\
\hline $10 / 25 / 73$ & & $0.3460 \mathrm{E}+00$ & $0.000 \mathrm{E}+00$
\end{tabular}

200 West Control Plot 60 6193 Depth B-12 in.

\begin{tabular}{llll} 
Sample & Dup. & Regult & $+/-2$ \\
Date & No. & pCi $/ g$ & std. Dev. \\
\hline $10 / 25 / 73$ & & $0.4050 \mathrm{E}+00$ & $0.000 \mathrm{E}+00$
\end{tabular}

other 200 Area Iocations

\begin{tabular}{llll}
$\begin{array}{l}\text { Inter. } 45 \text { and Army Loop Rd. } \\
6223 \\
\text { Depth } 0-1 \text { in. }\end{array}$ & Uranium \\
Sample & Dup. Regult & $+/-2$ \\
Date & No. & pCl/g & std. Dev. \\
\hline $08 / 10 / 76$ & & $0.2000 E+00$ & $0.000 E+00$ \\
$08 / 18 / 75$ & $0.3980 E+00$ & $0.000 E+00$ \\
$09 / 24 / 74$ & & $0.3520 E+00$ & $0.000 E+00$
\end{tabular}

ArTuY Loop Rd. Contl. Plot 40 6226 Depth $0-1$ in. Uranium

\begin{tabular}{llll} 
Sample & Dup. & Regult & $+/-2$ \\
Date & No. & pCi/g & std. Dev. \\
\hline $09 / 24 / 74$ & & $0.2450 \mathrm{E}+00$ & $0.000 \mathrm{E}+00$
\end{tabular}

Highway 240 Control Plot 54 6002 Depeh $0-1$ in. Uranium

\begin{tabular}{llll} 
Sample & Dup. & Regult & $+/-2$ \\
Date & No. & pc1/g & Std. Dev. \\
\hline $09 / 23 / 74$ & & $0.1820 \mathrm{E}+00$ & $0.000 \mathrm{E}+00$ \\
$10 / 25 / 73$ & & $0.3710 \mathrm{E}+00$ & $0.000 \mathrm{E}+00$
\end{tabular}

300 Area

200 Area Fire station

6021 Depth 0-1 in. Uranium

\begin{tabular}{llll} 
Sample & Dup. & Regult & $+/-2$ \\
Date & No. & pci/g & Std. Dev. \\
\hdashline $10 / 25 / 73$ & & $0.5550 \mathrm{E}+00$ & $0.000 \mathrm{E}+00$
\end{tabular}

Gable Pond Control plot 43

$\begin{array}{llll}6321 & \text { Depth } 0-1 \text { in. } & \text { Uranium } \\ \text { Sample } & \text { Dup. } & \text { Result } & +/-2 \\ \text { Date } & \text { No. } & \text { pCi/g } & \text { Std. Dev. } \\ -08 / 11 / 76 & & 0.2330 \mathrm{E}+00 & 0.000 \mathrm{E}+00\end{array}$

\begin{tabular}{llll}
$\begin{array}{l}\text { North of } \\
6322\end{array}$ & $\begin{array}{l}\text { 300 Area } \\
\text { Depth 0-1 1n. }\end{array}$ & Uraniur \\
& & & \\
Sample & Dup. Result & $+/-2$ \\
Date & No. & pCi/g & Std. Dev. \\
\hline $04 / 29 / 87$ & & $0.3830 E+01$ & $0.000 E+00$ \\
$11 / 25 / 86$ & & $0.6580 E+00$ & $0.000 E+00$ \\
$10 / 09 / 85$ & 1 & $0.2040 E+01$ & $0.580 E+00$ \\
$05 / 31 / 85$ & & $0.5440 E+01$ & $0.165 E+01$ \\
$05 / 16 / 84$ & & $0.7560 E+00$ & $0.204 E+00$ \\
$08 / 11 / 83$ & & $0.4990 E+00$ & $0.136 E+00$ \\
$09 / 03 / 82$ & & $0.9890 E+00$ & $0.346 E+00$ \\
$08 / 10 / 76$ & & $0.2590 E+00$ & $0.000 E+00$
\end{tabular}


TABLE A.7. (contd)

300 Area

North Center 300 Area Fence

Depth 0-1 in. Uranjum

\begin{tabular}{llll} 
Sample & Dup. & Result & $+/{ }^{2}$ \\
Date & No. & pCi/g & Std. Dev. \\
\hline $08 / 20 / 75$ & & $0.1270 E+01$ & $0.000 E+00$
\end{tabular}

\begin{tabular}{llll}
300 Area & $\begin{array}{l}\text { South Gate } \\
\text { Depth } 0-1 \text { in. }\end{array}$ & Uranlur \\
Sample & Dup. Result & $+/-2$ \\
Date & No. & pCi $/ g$ & Std. Dev. \\
\hline $09 / 24 / 74$ & & $0.6580 E+00$ & $0.000 E+00$
\end{tabular}

South of 300 Area

\begin{tabular}{llll}
6323 & Depth $0-1$ in. & Uranium \\
Sample & Dup. & Regult & $+/-2$ \\
Date & No. & pCi/g & Std. Dev. \\
\hline $04 / 29 / 86$ & & $0.5570 \mathrm{E}+00$ & $0.000 \mathrm{E}+00$ \\
$05 / 31 / 85$ & & $0.6630 \mathrm{E}+00$ & $0.206 \mathrm{E}+00$ \\
$05 / 16 / 84$ & & $0.1040 \mathrm{E}+01$ & $0.294 \mathrm{E}+00$ \\
$08 / 11 / 83$ & $0.3100 \mathrm{E}+00$ & $0.844 \mathrm{E}-01$ \\
$09 / 03 / 82$ & & $0.5060 \mathrm{E}+00$ & $0.177 \mathrm{E}+00$ \\
$08 / 10 / 76$ & & $0.2810 \mathrm{E}+00$ & $0.000 \mathrm{D}+00$
\end{tabular}

331 8ldg. Control Plot 50 6014 Depth $0-1$ in.

Uranium

Sample Dup. Result $t /-2$

Date No. pCi/g std. Dev.

- $\overline{08 / 20 / 75}-\overline{0.1740 \mathrm{E}+00} \quad \overline{0.000 \mathrm{E}+00}$

$10 / 25 / 73 \quad 0.26908+00 \quad 0.000 E+00$
400 Aree and Vicinity

\begin{tabular}{llll}
$\begin{array}{l}\text { Wye Barricade } \\
6016\end{array}$ & Depth & $0-1$ in. & Uranium \\
Sample & Dup. & Regult & $+/-2$ \\
Date & No. & pCi/g & Std. Dev. \\
\hline $04 / 29 / 87$ & & $0.1900 \mathrm{E}+00$ & $0.000 \mathrm{E}+00$ \\
$07 / 03 / 85$ & $0.2860 \mathrm{E}+00$ & $0.972 \mathrm{E}-01$ \\
$05 / 15 / 84$ & $0.6500 \mathrm{E}+00$ & $0.102 \mathrm{E}+00$ \\
$08 / 11 / 83$ & $0.1870 \mathrm{E}+00$ & $0.526 \mathrm{E}-01$ \\
$08 / 16 / 82$ & $0.3800 \mathrm{E}+00$ & $0.133 \mathrm{E}+00$ \\
$08 / 10 / 81$ & $0.3740 \mathrm{E}+00$ & $0.130 \mathrm{E}+00$ \\
$08 / 05 / 80$ & $0.2620 \mathrm{E}+00$ & $0.920 \mathrm{E}-01$ \\
$09 / 11 / 79$ & $0.2220 \mathrm{E}+00$ & $0.7 \mathrm{BOE}-01$ \\
$08 / 08 / 78$ & $0.1980 \mathrm{E}+00$ & $0.150 \mathrm{E}+00$ \\
$06 / 14 / 77$ & $0.1810 \mathrm{E}+00$ & $0.680 \mathrm{E}-01$ \\
$10 / 25 / 73$ & $0.1160 \mathrm{E}+00$ & $0.000 \mathrm{E}+00$
\end{tabular}

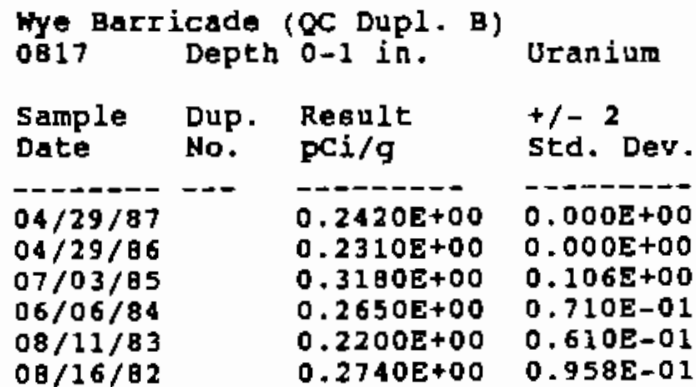

\begin{tabular}{|c|c|c|c|}
\hline $\begin{array}{l}1 \text { Mile } \\
6324\end{array}$ & $\begin{array}{l}\text { of } \\
\text { Dept }\end{array}$ & o-1 in. & Uranium \\
\hline $\begin{array}{l}\text { Sample } \\
\text { Date }\end{array}$ & $\begin{array}{l}\text { Dup. } \\
\text { No. }\end{array}$ & $\begin{array}{l}\text { Result } \\
\mathrm{pcl} / \mathrm{g}\end{array}$ & $\begin{array}{l}+/-2 \\
\text { std. Dev. }\end{array}$ \\
\hline $8 / 10 / 76$ & $-\infty$ & $0.1530 \mathrm{E}+00$ & $0.000 \mathrm{E}+00$ \\
\hline
\end{tabular}


TABLE_A.7. (contd)

400 Area and vicinity

Other onsite tocations

1 Mile $s$ of wPpSS No. 2 6325 Depth $0-1$ in.

\begin{tabular}{llll} 
Sample & Dup. & Regult & $+/-2$ \\
Date & No. & pC1/g & Std. Dev. \\
\hline $08 / 10 / 76$ & & $0.1570 \mathrm{E}+00$ & $0.000 \mathrm{E}+00$
\end{tabular}

FFTF Control Plot 62

$\begin{array}{llll}6015 & \text { Depth } 0-1 \text { in. } & \text { Uraniug } \\ \text { Sanple } & \text { Dup. } & \text { Result } & +/-2 \\ \text { Date } & \text { No. } & \text { pCl/g } & \text { Std. Dev. } \\ -09 / 24 / 74 & & 0.8510 \mathrm{E}+00 & 0.000 \mathrm{E}+00 \\ 10 / 25 / 73 & & 0.3540 \mathrm{E}+00 & 0.000 \mathrm{E}+00\end{array}$

0.5 Hile NE of FFTF

\begin{tabular}{llll}
6282 & Depth $0-1$ in. & Uranium \\
Sample & Dup. & Result & $+/-2$ \\
Date & No. & pC1/g & Std. Dev. \\
\hline $04 / 29 / 87$ & & $0.2390 \mathrm{E}+00$ & $0.000 \mathrm{E}+00$ \\
$06 / 05 / 85$ & & $0.3900 \mathrm{E}+00$ & $0.129 \mathrm{E}+00$ \\
$05 / 17 / 84$ & $0.2990 \mathrm{E}+00$ & $0.022 \mathrm{E}-01$ \\
$08 / 12 / 83$ & $0.2480 \mathrm{E}+00$ & $0.684 \mathrm{E}-01$ \\
$08 / 30 / 82$ & $0.2960 \mathrm{E}+00$ & $0.104 \mathrm{E}+00$ \\
$08 / 10 / 81$ & $0.4890 \mathrm{E}+00$ & $0.170 \mathrm{E}+00$ \\
$08 / 05 / 80$ & $0.2170 \mathrm{E}+00$ & $0.760 \mathrm{E}-01$ \\
$09 / 11 / 79$ & $0.2310 \mathrm{E}+00$ & $0.010 \mathrm{E}-01$ \\
$08 / 08 / 78$ & $0.2640 \mathrm{E}+00$ & $0.150 \mathrm{E}+00$ \\
$06 / 14 / 77$ & $0.2530 \mathrm{E}+00$ & $0.680 \mathrm{E}-01$ \\
$08 / 10 / 76$ & $0.1910 \mathrm{E}+00$ & $0.000 \mathrm{E}+00$ \\
$08 / 20 / 75$ & $0.1690 \mathrm{E}+00$ & $0.000 \mathrm{E}+00$
\end{tabular}

SE side of FFTF

\begin{tabular}{|c|c|c|c|}
\hline $\begin{array}{l}\text { Sample } \\
\text { Date }\end{array}$ & $\begin{array}{l}\text { Depth } \\
\text { Dup. } \\
\text { No. }\end{array}$ & $\begin{array}{l}\text { 0-1 in. } \\
\text { Result } \\
\text { pci/g }\end{array}$ & $\begin{array}{l}\text { Uranium } \\
+/ \vec{~}^{2} \\
\text { Std. Dev. }\end{array}$ \\
\hline $\begin{array}{l}-\overline{0}---1- \\
04 / 29 / 86 \\
06 / 05 / 85 \\
05 / 17 / 84 \\
08 / 12 / 83 \\
08 / 30 / 82 \\
08 / 10 / 81 \\
08 / 05 / 80 \\
09 / 11 / 79 \\
08 / 08 / 78 \\
06 / 14 / 77 \\
08 / 20 / 75\end{array}$ & --- & $\begin{array}{l}\overline{0}-3740 \mathrm{E}+00 \\
0.4040 \mathrm{E}+00 \\
0.2700 \mathrm{E}+00 \\
0.1620 \mathrm{E}+00 \\
0.2790 \mathrm{E}+00 \\
0.4650 \mathrm{E}+00 \\
0.2300 \mathrm{E}+00 \\
0.3830 \mathrm{E}+00 \\
0.2480 \mathrm{E}+00 \\
0.2990 \mathrm{E}+00 \\
0.1700 \mathrm{0}+00\end{array}$ & $\begin{array}{l}--. \\
0.000 \mathrm{E}+00 \\
0.132 \mathrm{E}+00 \\
0.726 \mathrm{E}-01 \\
0.464 \mathrm{E} \sim 01 \\
0.97 \mathrm{E}-01 \\
0.160 \mathrm{E}+00 \\
0.010 \mathrm{0}-01 \\
0.130 \mathrm{E}+00 \\
0.150 \mathrm{0}+00 \\
0.680 \mathrm{0}-01 \\
0.000 \mathrm{E}+00\end{array}$ \\
\hline
\end{tabular}

$N$ End of Vernita Bridge

6005 Depch 0-1 in. Uranium

\begin{tabular}{|c|c|c|c|}
\hline $\begin{array}{l}\text { Sample } \\
\text { Date }\end{array}$ & $\begin{array}{l}\text { Dup. } \\
\text { No. }\end{array}$ & $\begin{array}{l}\text { Regult } \\
\mathrm{pCi} / \mathrm{g}\end{array}$ & $\begin{array}{l}+/{ }^{2} \\
\text { Std. Dev. }\end{array}$ \\
\hline $\begin{array}{l}05 / 02 / 86 \\
06 / 11 / 85 \\
05 / 31 / 84 \\
08 / 11 / 83 \\
08 / 24 / 82 \\
09 / 23 / 74 \\
10 / 25 / 73\end{array}$ & --- & $\begin{array}{l}\overline{0.3440 E+00} \\
0.7260 \mathrm{E}+00 \\
0.9240 \mathrm{E}+00 \\
0.3700 \mathrm{E}+00 \\
0.37 \mathrm{BOE}+00 \\
0.1320 \mathrm{E}+00 \\
0.2790 \mathrm{E}+00\end{array}$ & $\begin{array}{l}0.000 \mathrm{E}+00 \\
0.222 \mathrm{E}+00 \\
0.260 \mathrm{E}+00 \\
0.102 \mathrm{E}+00 \\
0.132 \mathrm{E}+00 \\
0.000 \mathrm{E}+00 \\
0.000 \mathrm{E}+00\end{array}$ \\
\hline
\end{tabular}

Hanford Townsite

6017 Depth 0-1 in. Uranium

Sample Dup. Regult $t /-2$

Date Ho. pCi/g std. Dev.

$\overline{04 / 29 / 87}=-\quad \overline{0.4200 \mathrm{E}+00} \quad \overline{0.000 \mathrm{E}+00}$

$06 / 12 / 85 \quad 0.3510 E+00 \quad 0.117 E+00$

$05 / 17 / 84 \quad 0.3430 \mathrm{E}+00 \quad 0.934 E-01$

$08 / 12 / 83 \quad 0.2390 \mathrm{E}+00 \quad 0.670 \mathrm{E}-01$

$08 / 30 / 82 \quad 0.3240 E+00 \quad 0.113 E+00$

$08 / 10 / \theta 1 \quad 0.4010 E+00 \quad 0.140 E+00$

$08 / 05 / 80 . \quad 0.2800 \mathrm{E}+00 \quad 0.980 \mathrm{E}-01$

$09 / 11 / 79 \quad 0.3650 E+00 \quad 0.130 E+00$

$08 / 08 / 78 \quad 0.3500 E+00 \quad 0.150 E+00$

$06 / 14 / 77 \quad 0.1880 E+00 \quad 0.680 E-01$

$08 / 11 / 76 \quad 0.3260 E+00 \quad 0.000 E+00$

$08 / 18 / 75 \quad 0.2410 \mathrm{E}+00 \quad 0.000 \mathrm{E}+00$

$10 / 25 / 73 \quad 0.3020 \mathrm{E}+00 \quad 0.000 \mathrm{E}+00$

\begin{tabular}{|c|c|c|c|}
\hline $\begin{array}{l}\text { Prosger } \\
5225\end{array}$ & $\begin{array}{c}\text { Barrice } \\
\text { Depth }\end{array}$ & $\begin{array}{l}\text { de } \\
0-1 \text { in. }\end{array}$ & Uranium \\
\hline $\begin{array}{l}\text { Sample } \\
\text { Date }\end{array}$ & $\begin{array}{l}\text { Dup. } \\
\text { No. }\end{array}$ & $\begin{array}{l}\text { Result } \\
\mathrm{pCi} / \mathrm{g}\end{array}$ & $\begin{array}{l}+/-2 \\
\text { std. Dev. }\end{array}$ \\
\hline $\begin{array}{l}04 / 29 / 86 \\
04 / 06 / 85 \\
06 / 05 / 15 / 84 \\
08 / 23 / 83 \\
06 / 16 / 82 \\
08 / 11 / 81 \\
08 / 04 / 80 \\
09 / 12 / 79 \\
08 / 08 / 78 \\
06 / 14 / 77 \\
08 / 19 / 75 \\
09 / 24 / 74\end{array}$ & $=-$ & $\begin{array}{l}-.2540 \mathrm{E}+00 \\
0.7980 \mathrm{E}+00 \\
0.3600 \mathrm{E}+00 \\
0.2490 \mathrm{E}+00 \\
0.2010 \mathrm{E}+00 \\
0.3500 \mathrm{E}+00 \\
0.1620 \mathrm{E}+00 \\
0.3010 \mathrm{E}+00 \\
0.6790 \mathrm{E}+00 \\
0.3180 \mathrm{E}+00 \\
0.2060 \mathrm{E}+00 \\
0.1320 \mathrm{E}+00\end{array}$ & $\begin{array}{l}0.000 \mathrm{E}+00 \\
0.242 \mathrm{E}+00 \\
0.640 \mathrm{E}-01 \\
0.686 \mathrm{E}-01 \\
0.703 \mathrm{E}-01 \\
0.120 \mathrm{E}+00 \\
0.570 \mathrm{E}-01 \\
0.110 \mathrm{E}+00 \\
0.150 \mathrm{E}+00 \\
0.680 \mathrm{E}-01 \\
0.000 \mathrm{E}+00 \\
0.000 \mathrm{E}+00\end{array}$ \\
\hline
\end{tabular}


TABLE A.7. (contd)

other Onsite tocations

\begin{tabular}{llll} 
ERC (Emer. Reloc. Center) & \\
6001 & Depth $0-1$ in. & Uranium \\
Sample & Dup. & Result & $+/-2$ \\
Date & No. & pCi $/ g$ & std. Dev. \\
\hline $09 / 24 / 74$ & & $0.7030 E+00$ & $0.000 E+00$ \\
$10 / 25 / 73$ & & $0.3910 E+00$ & $0.000 E+00$
\end{tabular}

$E$ of Arid Land Ecol. Lab

\begin{tabular}{llll}
6278 & Depth $0-1$ in. & Uranium \\
Sample & Dup. & Result & $+/-2$ \\
Date & No. & pC1/g & std. Dev. \\
\hline $05 / 20 / 87$ & & $0.4530 E+00$ & $0.000 E+00$ \\
$06 / 12 / 85$ & & $0.4560 \mathrm{E}+00$ & $0.146 \mathrm{E}+00$ \\
$05 / 24 / 84$ & $0.2460 \mathrm{E}+00$ & $0.670 \mathrm{E}-01$ \\
$08 / 23 / 83$ & $0.2830 \mathrm{E}+00$ & $0.780 \mathrm{E}-01$ \\
$08 / 16 / 82$ & $0.3480 \mathrm{E}+00$ & $0.122 \mathrm{E}+00$ \\
$08 / 13 / 81$ & $0.3130 \mathrm{E}+00$ & $0.110 \mathrm{E}+00$ \\
$08 / 05 / 80$ & $0.3630 \mathrm{E}+00$ & $0.130 \mathrm{E}+00$ \\
$09 / 12 / 79$ & $0.3300 \mathrm{E}+00$ & $0.120 \mathrm{E}+00$ \\
$08 / 10 / 78$ & $0.3820 \mathrm{E}+00$ & $0.150 \mathrm{E}+00$ \\
$06 / 14 / 77$ & $<0.4570 \mathrm{E}-01$ & $0.680 \mathrm{E}-01$ \\
$00 / 10 / 76$ & $0.2850 \mathrm{E}+00$ & $0.000 \mathrm{E}+00$ \\
$08 / 20 / 75$ & $0.1520 \mathrm{E}+00$ & $0.000 \mathrm{E}+00$
\end{tabular}

$\begin{array}{llll}\begin{array}{l}\text { Yakima Barricade } \\ 6004 \\ \text { Deptb } 0-1 \text { in. }\end{array} & \text { Uranium } \\ \text { Sample } & \text { Dup. } & \text { Regult } & +/-2 \\ \text { Date } & \text { No. } & \text { pCi/g } & \text { std. Dev. } \\ - & & \\ 05 / 07 / 87 & & 0.2710 \mathrm{E}+00 & 0.000 \mathrm{E}+00 \\ 06 / 06 / 85 & 0.3460 \mathrm{E}+00 & 0.115 \mathrm{E}+00 \\ 05 / 24 / 84 & 0.2060 \mathrm{E}+00 & 0.562 \mathrm{E}-01 \\ 08 / 11 / 83 & 0.2550 \mathrm{E}+00 & 0.706 \mathrm{E}-01 \\ 08 / 17 / 82 & 0.2270 \mathrm{E}+00 & 0.794 \mathrm{E}-01 \\ 08 / 11 / 81 & 0.5000 \mathrm{E}+00 & 0.170 \mathrm{E}+00 \\ 08 / 04 / 80 & 0.2910 \mathrm{E}+00 & 0.100 \mathrm{E}+00 \\ 09 / 11 / 79 & 0.3890 \mathrm{E}+00 & 0.140 \mathrm{E}+00 \\ 08 / 08 / 78 & 0.2140 \mathrm{E}+00 & 0.150 \mathrm{E}+00 \\ 06 / 14 / 77 & 0.1230 \mathrm{E}+00 & 0.680 \mathrm{E}-01 \\ 08 / 10 / 76 & 0.2440 \mathrm{E}+00 & 0.000 \mathrm{E}+00 \\ 08 / 19 / 75 & 0.2530 \mathrm{E}+00 & 0.000 \mathrm{E}+00 \\ 09 / 23 / 74 & 0.1010 \mathrm{E}+00 & 0.000 \mathrm{E}+00 \\ 10 / 25 / 73 & 0.3040 \mathrm{E}+00 & 0.000 \mathrm{E}+00\end{array}$

Rattlesnake Springs

\begin{tabular}{|c|c|c|c|}
\hline 003 & Depth & $0-1$ in. & Uranium \\
\hline $\begin{array}{l}\text { ample } \\
\text { ate }\end{array}$ & $\begin{array}{l}\text { Dup. } \\
\text { No. }\end{array}$ & $\begin{array}{l}\text { Rosult } \\
\mathrm{pCi} / \mathrm{g}\end{array}$ & $\begin{array}{l}+/-2 \\
\text { std. Dev. }\end{array}$ \\
\hline $\begin{array}{l}05 / 07 / 87 \\
05 / 23 / 86 \\
06 / 06 / 85 \\
05 / 24 / 84 \\
00 / 11 / 83 \\
09 / 02 / 82 \\
09 / 23 / 74 \\
10 / 25 / 73\end{array}$ & --- & $\begin{array}{l}0.2780 \mathrm{E}+00 \\
0.2610 \mathrm{E}+00 \\
0.4410 \mathrm{E}+00 \\
0.2580 \mathrm{E}+00 \\
0.2510 \mathrm{E}+00 \\
0.3000 \mathrm{E}+00 \\
0.3210 \mathrm{E}+00 \\
0.5170 \mathrm{E}+00\end{array}$ & $\begin{array}{l}0.000 \mathrm{E}+00 \\
0.000 \mathrm{E}+00 \\
0.143 \mathrm{E}+00 \\
0.692 \mathrm{E}-01 \\
0.702 \mathrm{E}-01 \\
0.105 \mathrm{E}+00 \\
0.000 \mathrm{E}+00 \\
0.000 \mathrm{E}+00\end{array}$ \\
\hline
\end{tabular}


APPENDIX B

OFFSITE ROUTINE SOIL SAMPLING LOCATIONS AND RESULTS 
IABLE B.1. Offsite Routine Soil Sampling Locations, 1971 through 1987

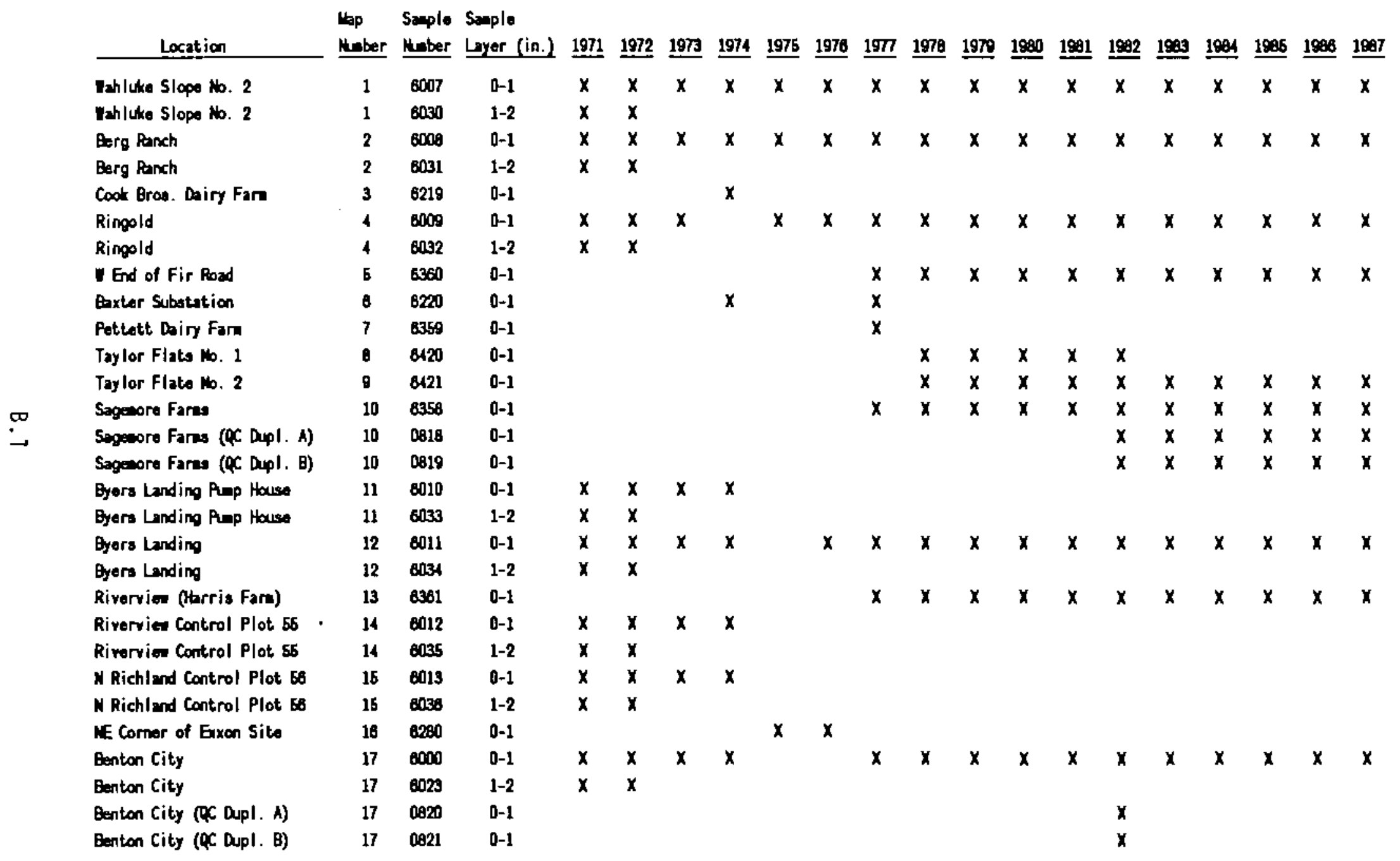


IABLE B.1. (contd)

tha Sauple Sample

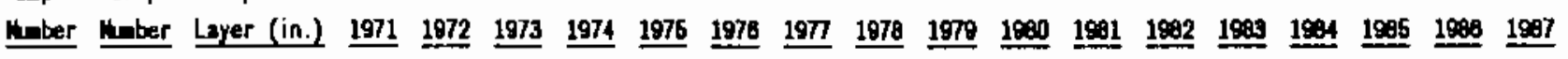

Sunnygide

Yakien

theses Lake

Othello

Connell

lashtucna

Valla Dalla

Lithery Das
$18 \quad 0357 \quad 0-1$

$18 \quad 0512 \quad 0-1$

$200500 \quad 0-1$

$210505 \quad 0-1$

$220503 \quad 0-1$

$230501 \quad 0-1$

$24 \quad 0502 \quad 0-1$

$250504 \quad 0-1$ $\begin{array}{lllllll} & \times & \times & \times & \times & x & x\end{array}$

$\begin{array}{ccc}x & x & x \\ & x & x \\ & x & x \\ & x & x \\ & x & x \\ & x & x \\ x & x & x \\ x & x & x\end{array}$




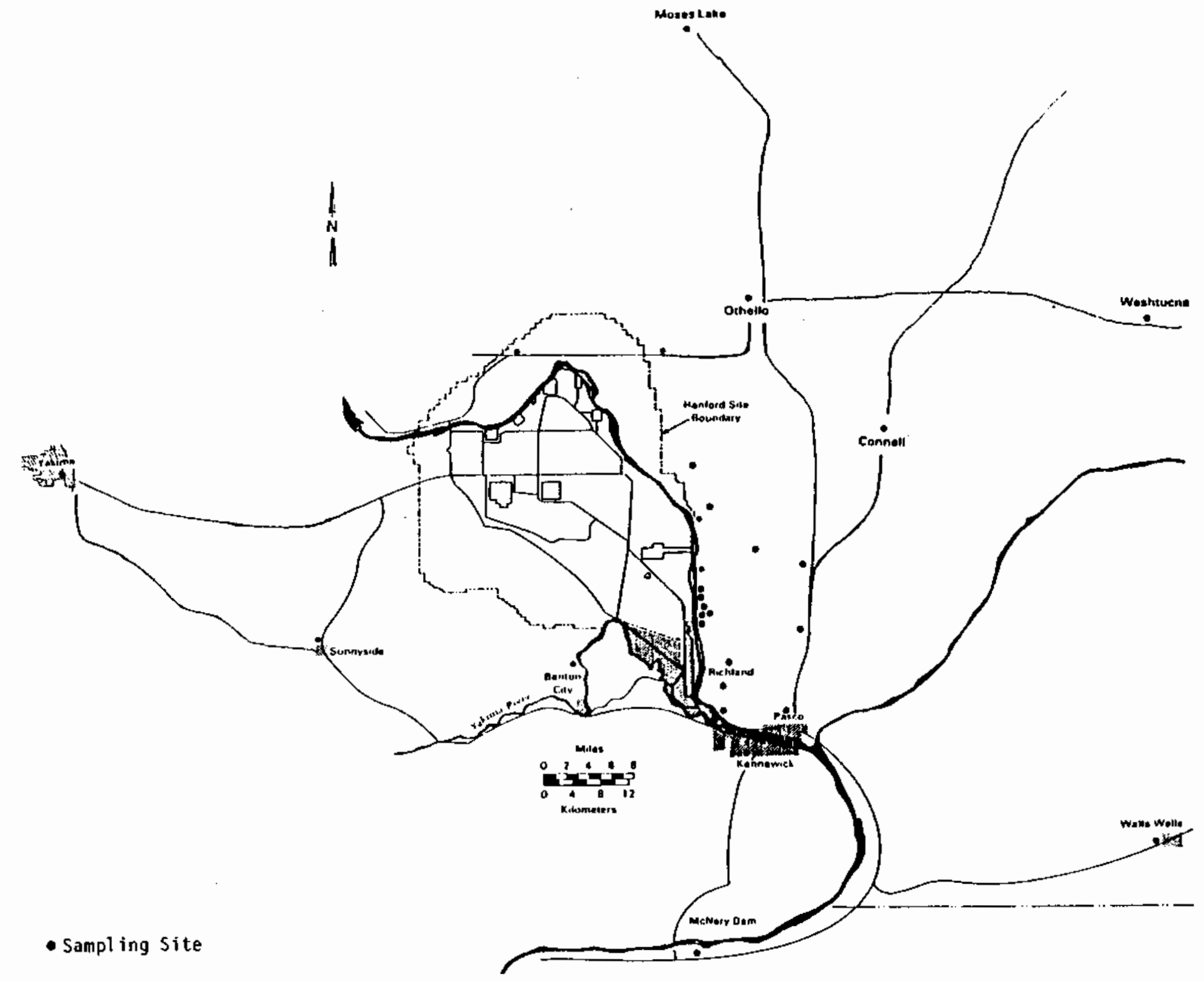

FIGURE B.1. Offsite Routine Soil Sampling Locations, 1971 to 1987 
Wahluke Slope No. 2

\begin{tabular}{llll}
6007 & Depth $0-1$ in. & Sr-90 \\
Sample & Dup. & Regult & $+/-2$ \\
Date & No. & pCi/g & std. Dev. \\
\hline $05 / 26 / 87$ & & $0.7470 \mathrm{E}-01$ & $0.101 \mathrm{E}-01$ \\
$05 / 02 / 86$ & & $0.9830 \mathrm{E}-01$ & $0.656 \mathrm{E}-02$ \\
$06 / 12 / 85$ & $0.2070 \mathrm{E}+00$ & $0.227 \mathrm{E}-01$ \\
$05 / 31 / 84$ & $0.1610 \mathrm{E}+00$ & $0.170 \mathrm{E}-01$ \\
$0 \mathrm{~B} / 26 / 83$ & $0.6460 \mathrm{E}+00$ & $0.232 \mathrm{E}-01$ \\
$0 \mathrm{~B} / 30 / 82$ & $0.9920 \mathrm{E}-01$ & $0.349 \mathrm{E}-01$ \\
$08 / 11 / 81$ & $0.8990 \mathrm{E}-01$ & $0.220 \mathrm{E}-02$ \\
$08 / 06 / 80$ & $0.3090 \mathrm{E}+00$ & $0.140 \mathrm{E}-01$ \\
$09 / 13 / 79$ & $0.9580 \mathrm{E}-01$ & $0.670 \mathrm{E}-02$ \\
$08 / 09 / 78$ & $0.1140 \mathrm{E}-01$ & $0.340 \mathrm{E}-02$ \\
$06 / 14 / 77$ & $0.1670 \mathrm{E}-01$ & $0.340 \mathrm{E}-02$ \\
$08 / 09 / 76$ & $0.3090 \mathrm{E}-01$ & $0.000 \mathrm{E}+00$ \\
$08 / 19 / 75$ & $0.1000 \mathrm{E}+00$ & $0.000 \mathrm{E}+00$ \\
$09 / 23 / 74$ & $0.1540 \mathrm{E}-01$ & $0.000 \mathrm{E}+00$ \\
$10 / 25 / 73$ & $0.3740 \mathrm{E}+00$ & $0.540 \mathrm{E}-02$ \\
$10 / 25 / 72$ & $0.2950 \mathrm{E}+00$ & $0.000 \mathrm{E}+00$ \\
$09 / 29 / 71$ & $0.1830 \mathrm{E}+00$ & $0.000 \mathrm{E}+00$
\end{tabular}

Wahluke slope No. 2

$\begin{array}{llll}6030 & \text { Depth } & 1-2 \text { in. } & 5 r-90 \\ \text { Sample } & \text { Dup. } & \text { Result } & +/-2 \\ \text { Date } & \text { No. } & \text { pCi/g } & \text { std. Dev. } \\ -70 / 25 / 72 & & 0.1310 E+00 & 0.000 E+00 \\ 09 / 29 / 71 & & 0.9200 E-01 & 0.000 E+00\end{array}$

Berg Ranch

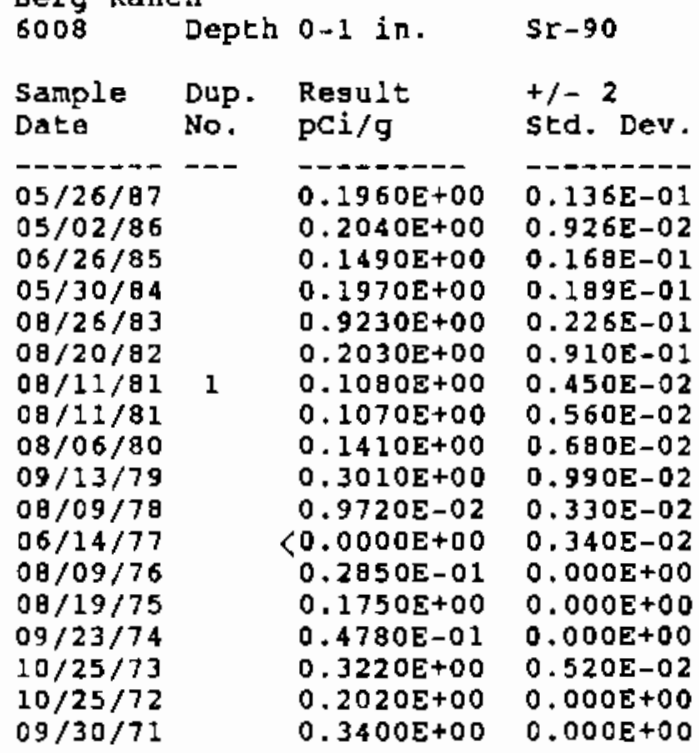

Berg Ranth

\begin{tabular}{llll}
6031 & Depth $1-2$ in. & Sr-90 \\
Sample & Dup. & Result & $+/-2$ \\
Date & No. & pCi/g & Std. Dev. \\
\hline $10 / 25 / 72$ & & $0.7000 E-01$ & $0.000 E+00$ \\
$09 / 29 / 71$ & & $0.3400 E+00$ & $0.000 E+00$
\end{tabular}

Cook Bros, Dairy Farm

\begin{tabular}{llll}
6219 & Depth 0-1 in. & Sr-90 \\
Sample & Dup. Result & $+/-2$ \\
Date & No. & pCi/g & Std. Dev. \\
\hdashline $09 / 25 / 74$ & & $0.5910 \mathrm{DE}-01$ & $0.000 \mathrm{E}+00$
\end{tabular}

Ringold

\begin{tabular}{|c|c|c|c|}
\hline 6009 & Depth & $0-1$ in. & $S I-90$ \\
\hline $\begin{array}{l}\text { ample } \\
\text { ate }\end{array}$ & $\begin{array}{l}\text { Dup. } \\
\text { No. }\end{array}$ & $\begin{array}{l}\text { Result } \\
\mathrm{pCi} / \mathrm{g}\end{array}$ & $\begin{array}{l}+/-2 \\
\text { std. Dev. }\end{array}$ \\
\hline $\begin{array}{l}-05 / 15 / 87 \\
05 / 22 / 86 \\
07 / 08 / 85 \\
05 / 30 / 84 \\
08 / 18 / 83 \\
08 / 20 / 82 \\
08 / 12 / 81 \\
08 / 06 / 80 \\
09 / 13 / 79 \\
08 / 09 / 78 \\
06 / 14 / 77 \\
08 / 09 / 76 \\
08 / 19 / 75 \\
10 / 25 / 73 \\
10 / 25 / 72 \\
10 / 01 / 71\end{array}$ & $--\infty$ & $\begin{array}{l}0.2080 \mathrm{E}+00 \\
0.2620 \mathrm{E}+00 \\
0.1980 \mathrm{E}+00 \\
0.2410 \mathrm{E}+00 \\
0.1750 \mathrm{E}+01 \\
0.84 \mathrm{~B} 0 \mathrm{E}-01 \\
0.2000 \mathrm{E}+00 \\
0.6960 \mathrm{E}-01 \\
0.1770 \mathrm{E}+00 \\
0.2560 \mathrm{E}-01 \\
<0.3320 \mathrm{E}-02 \\
0.1720 \mathrm{E}-01 \\
0.7290 \mathrm{E}-01 \\
0.3810 \mathrm{E}-01 \\
0.1170 \mathrm{E}+00 \\
0.1180 \mathrm{E}+00\end{array}$ & $\begin{array}{l}-.140 \mathrm{E}-01 \\
0.144 \mathrm{E}-01 \\
0.247 \\
0.179 \mathrm{E}-01 \\
0.143 \mathrm{E}-01 \\
0.321 \mathrm{E}-01 \\
0.390 \mathrm{E}-01 \\
0.220 \mathrm{E}-02 \\
0.680 \mathrm{E}-02 \\
0.120 \mathrm{E}-01 \\
0.360 \mathrm{E}-02 \\
0.340 \mathrm{E}-02 \\
0.000 \mathrm{E}+00 \\
0.000 \mathrm{E}+00 \\
0.230 \mathrm{E}-02 \\
0.000 \mathrm{E}+00 \\
0.000 \mathrm{E}+00\end{array}$ \\
\hline
\end{tabular}

\begin{tabular}{llll}
$\begin{array}{l}\text { Ringold } \\
6032\end{array}$ & Depth & $1-2$ in. & Sr-90 \\
Sample & Dup. & Result & $+/-2$ \\
Date & No. & pCi/g & std. Dev. \\
\hdashline $10 / 25 / 72$ & & $0.7970 E-01$ & $0.000 E+00$ \\
$10 / 01 / 71$ & & $0.9950 E+00$ & $0.000 E+00$
\end{tabular}


TABLE B.2. (contd)

$\begin{array}{llll}\begin{array}{l}\text { W End of Fir Road } \\ 6360\end{array} & \begin{array}{l}\text { Depth } 0-1 \text { in. } \\ \text { Sr-90 }\end{array} \\ \text { Sample } & \text { Dup. } & \text { Result } & +/-2 \\ \text { Date } & \text { No. } & \text { pCi/g } & \text { std. Dev. } \\ & & & \\ 05 / 15 / 87 & & 0.5360 E-01 & 0.780 E-02 \\ 05 / 22 / 86 & 0.1200 E+00 & 0.178 E-01 \\ 07 / 03 / 85 & 0.9110 E-01 & 0.121 E-01 \\ 05 / 31 / 84 & 0.1430 E+00 & 0.151 E-01 \\ 08 / 18 / 83 & 0.1180 E+01 & 0.311 E-01 \\ 08 / 20 / 82 & 0.7010 E-01 & 0.675 E-02 \\ 08 / 12 / 81 & 0.5060 E-01 & 0.310 E-02 \\ 08 / 06 / 80 & 0.1710 E+00 & 0.680 E-02 \\ 09 / 13 / 79 & 0.1210 E+00 & 0.890 E-02 \\ 08 / 09 / 78 & 0.1070 E-01 & 0.320 E-02 \\ 06 / 14 / 77 & 0.1500 E+00 & 0.770 E-02\end{array}$

\begin{tabular}{|c|c|c|}
\hline $\begin{array}{l}\text { Baxter } 5 \\
6220\end{array}$ & $\begin{array}{l}\text { ubstat } \\
\text { Depth }\end{array}$ & ion in. \\
\hline $\begin{array}{l}\text { Sample } \\
\text { Date }\end{array}$ & $\begin{array}{l}\text { Dup. } \\
\text { No. }\end{array}$ & $\begin{array}{l}\text { Result } \\
\text { pCi/g }\end{array}$ \\
\hline $\begin{array}{l}06 / 14 / 77 \\
09 / 25 / 74\end{array}$ & $=-0$ & $0.3040 \mathrm{E}-01$ \\
\hline
\end{tabular}

\begin{tabular}{|c|c|c|c|}
\hline $\begin{array}{l}\text { fectett } \\
6359\end{array}$ & $\begin{array}{l}\text { Dasry } \\
\text { Depth }\end{array}$ & Farm $0-1 \mathrm{n}$. & Sr -90 \\
\hline $\begin{array}{l}\text { Sample } \\
\text { Date }\end{array}$ & $\begin{array}{l}\text { Dup. } \\
\text { No. }\end{array}$ & $\begin{array}{l}\text { Result } \\
\mathrm{pCi} / \mathrm{g}\end{array}$ & $\begin{array}{l}+/-2 \\
\text { std. Dev. }\end{array}$ \\
\hline $06 / 14 / 77$ & -- & $\langle 0.2650 \mathrm{E}-02$ & $0.340 \mathrm{E}-02$ \\
\hline
\end{tabular}

Taylor Flata No. 1

\begin{tabular}{|c|c|c|c|}
\hline 120 & Depth & $0-1$ in. & Sr-90 \\
\hline $\begin{array}{l}\text { Sample } \\
\text { Date }\end{array}$ & $\begin{array}{l}\text { Dup. } \\
\text { No. }\end{array}$ & $\begin{array}{l}\text { Regult } \\
\mathrm{pCi} / \mathrm{g}\end{array}$ & $\begin{array}{l}+/-2 \\
\text { std. Dev. }\end{array}$ \\
\hline $\begin{array}{l}-0----7 \\
08 / 18 / 82 \\
00 / 14 / 81 \\
01 / 22 / 81 \\
08 / 06 / 80 \\
09 / 13 / 79 \\
0 \theta / 10 / 78\end{array}$ & --- & $\begin{array}{l}0.6910 \mathrm{E}-01 \\
0.1110 \mathrm{E}+00 \\
0.6640 \mathrm{E}-02 \\
0.7430 \mathrm{E}-02 \\
0.5520 \mathrm{E}-01 \\
0.7790 \mathrm{E}-02\end{array}$ & $\begin{array}{l}0.196 \mathrm{E}-01 \\
0.110 \mathrm{E}-01 \\
0.680 \mathrm{E}-02 \\
0.680 \mathrm{E}-02 \\
0.660 \mathrm{E}-02 \\
0.320 \mathrm{E}-02\end{array}$ \\
\hline
\end{tabular}

$5 r-90$

$+/-2$

std. Dev.

$0.000 E+00$

$0.340 \mathrm{E}-02$
Taylor Flatg No. 2

6421 Depth 0-1 in.

Sr -90

\begin{tabular}{|c|c|c|c|}
\hline ample & $\begin{array}{l}\text { Dup. } \\
\text { No. }\end{array}$ & $\begin{array}{l}\text { Result } \\
\mathrm{pCi} / \mathrm{g}\end{array}$ & $\begin{array}{l}+/-2 \\
\text { std. Dev. }\end{array}$ \\
\hline $\begin{array}{l}-\overline{05 / 15 / 87} \\
05 / 22 / 86 \\
07 / 08 / 85 \\
05 / 30 / 84 \\
08 / 26 / 83 \\
08 / 20 / 82 \\
11 / 03 / 81 \\
11 / 03 / 81 \\
11 / 03 / 81 \\
0 \theta / 14 / 81 \\
01 / 22 / 81 \\
08 / 06 / 80 \\
09 / 13 / 79 \\
08 / 10 / 7 \theta\end{array}$ & $\begin{array}{l}2 \\
1\end{array}$ & $\begin{array}{l}0.9740 E-01 \\
0.974 \\
0.3550 E+00 \\
0.4550 E-01 \\
0.4190 E-01 \\
0.2310 E+01 \\
0.2340 E+00 \\
0.1250 E+00 \\
0.6450 E-01 \\
0.1100 E+00 \\
0.2530 E+00 \\
0.1900 E+00 \\
0.2530 E+00 \\
0.3360 E+00 \\
0.6530 E-02\end{array}$ & $\begin{array}{l}-.101 \mathrm{E}-01 \\
0.191 \mathrm{E}-01 \\
0.1910 \\
0.874 \mathrm{E}-02 \\
0.821 \mathrm{E}-02 \\
0.387 \mathrm{E}-01 \\
0.591 \mathrm{E}-01 \\
0.260 \mathrm{E}-02 \\
0.310 \mathrm{E}-02 \\
0.110 \mathrm{E}-01 \\
0.890 \mathrm{E}-02 \\
0.200 \mathrm{E}-01 \\
0.110 \mathrm{E}-01 \\
0.990 \mathrm{E}-02 \\
0.340 \mathrm{E}-02\end{array}$ \\
\hline
\end{tabular}

$0.360 \mathrm{E}-02$

Sagemore Farms

\begin{tabular}{|c|c|c|c|}
\hline 050 & Depth & $0-1$ in. & Sr -90 \\
\hline $\begin{array}{l}\text { ample } \\
\text { ate }\end{array}$ & $\begin{array}{l}\text { Dup. } \\
\text { No. }\end{array}$ & $\begin{array}{l}\text { Regult } \\
\mathrm{pCi} / \mathrm{g}\end{array}$ & $\begin{array}{l}+/-2 \\
\text { std. Dev. }\end{array}$ \\
\hline $\begin{array}{l}-\infty----- \\
05 / 15 / 87 \\
05 / 22 / 86 \\
07 / 08 / 85 \\
05 / 30 / 84 \\
08 / 25 / 83 \\
08 / 18 / 82 \\
11 / 02 / 81 \\
08 / 12 / 81 \\
0 \theta / 06 / 80 \\
09 / 13 / 79 \\
00 / 09 / 78 \\
06 / 14 / 77\end{array}$ & -- & $\begin{array}{l}-.-\overline{0} \\
0.4100 \mathrm{E}-01 \\
0.1070 \mathrm{E}+00 \\
0.8060 \mathrm{E}-01 \\
0.2510 \mathrm{E}+00 \\
0.2770 \mathrm{E}+00 \\
0.6280 \mathrm{E}-01 \\
0.6140 \mathrm{E}-02 \\
0.1770 \mathrm{E}-01 \\
0.1550 \mathrm{E}-01 \\
0.2920 \mathrm{E}-01 \\
0.8000 \mathrm{E}-02 \\
0.1130 \mathrm{E}-01\end{array}$ & $\begin{array}{l}0.759 \mathrm{E}-02 \\
0.170 \mathrm{E}-01 \\
0.114 \mathrm{E}-01 \\
0.463 \mathrm{E}-01 \\
0.170 \mathrm{E}-01 \\
0.198 \mathrm{E}-01 \\
0.290 \mathrm{E}-02 \\
0.210 \mathrm{E}-02 \\
0.660 \mathrm{E}-02 \\
0.670 \mathrm{E}-02 \\
0.330 \mathrm{E}-02 \\
0.340 \mathrm{E}-02\end{array}$ \\
\hline
\end{tabular}

\begin{tabular}{|c|c|c|c|}
\hline $\begin{array}{l}\text { Sagemore } \\
0818\end{array}$ & $\begin{array}{l}\text { Farms } \\
\text { Depth }\end{array}$ & $\begin{array}{l}\text { LQC Dupl. } \\
\text { O-1 in. }\end{array}$ & Sr-90 \\
\hline $\begin{array}{l}\text { ample } \\
\text { ate }\end{array}$ & $\begin{array}{l}\text { Dup. } \\
\text { No. }\end{array}$ & $\begin{array}{l}\text { Result } \\
\mathrm{pCi} / \mathrm{g}\end{array}$ & $\begin{array}{l}+/-2 \\
\text { Std. Dev. }\end{array}$ \\
\hline $\begin{array}{l}-\overline{0}-1-1 / 15 \\
05 / 15 / 87 \\
05 / 22 / 86 \\
07 / 08 / 85 \\
05 / 30 / 84 \\
08 / 25 / 83 \\
0 \theta / 18 / 82\end{array}$ & --- & $\begin{array}{l}0.8940 \mathrm{E}-01 \\
0.1320 \mathrm{E}+00 \\
0.6110 \mathrm{E}-01 \\
0.1580 \mathrm{E}+00 \\
0.4470 \mathrm{E}+00 \\
0.5680 \mathrm{E}-01\end{array}$ & $\begin{array}{l}0.116 \mathrm{E}-01 \\
0.110 \mathrm{E}-01 \\
0.100 \mathrm{E}-01 \\
0.169 \mathrm{E}-01 \\
0.301 \mathrm{E}-01 \\
0.637 \mathrm{E}-02\end{array}$ \\
\hline
\end{tabular}


TABLE B.2. (contd)

\begin{tabular}{|c|c|c|c|}
\hline $\begin{array}{l}\text { 5agemoor } \\
0819\end{array}$ & $\begin{array}{l}\text { Farms } \\
\text { Depth }\end{array}$ & $\begin{array}{l}\text { KOC Dupl. } \\
\text { o-1 in. }\end{array}$ & $S r-90$ \\
\hline $\begin{array}{l}\text { Sample } \\
\text { Date }\end{array}$ & $\begin{array}{l}\text { Dup. } \\
\text { No. }\end{array}$ & $\begin{array}{l}\text { Result } \\
\mathrm{pC} i / \mathrm{g}\end{array}$ & $\begin{array}{l}+/ \overrightarrow{.}^{2} \\
\text { std. Dev. }\end{array}$ \\
\hline $\begin{array}{l}------- \\
05 / 15 / 87 \\
05 / 22 / 86 \\
07 / 08 / 85 \\
05 / 30 / 84 \\
08 / 25 / 83 \\
08 / 18 / 82\end{array}$ & --- & $\begin{array}{l}-.6440 \mathrm{E}-01 \\
0.61 \\
0.1300 \mathrm{E}+00 \\
0.6340 \mathrm{E}-01 \\
0.1750 \mathrm{E}+00 \\
0.4100 \mathrm{E}+00 \\
0.5300 \mathrm{E}-01\end{array}$ & $\begin{array}{l}0.106 \mathrm{E}-01 \\
0.990 \mathrm{E}-02 \\
0.122 \mathrm{E}-01 \\
0.273 \mathrm{E}-01 \\
0.248 \mathrm{E}-01 \\
0.129 \mathrm{E}-01\end{array}$ \\
\hline
\end{tabular}

\begin{tabular}{|c|c|c|c|}
\hline $\begin{array}{l}\text { Oyers Lan } \\
6010\end{array}$ & $\begin{array}{l}\text { nding } \\
\text { Depth }\end{array}$ & $\begin{array}{l}\text { Pump House } \\
0-1 \text { in. }\end{array}$ & $S I-90$ \\
\hline $\begin{array}{l}\text { Sample } \\
\text { Date }\end{array}$ & $\begin{array}{l}\text { Dup. } \\
\text { No. }\end{array}$ & $\begin{array}{l}\text { Result } \\
\text { pCi/g }\end{array}$ & $\begin{array}{l}+/{ }^{2} \\
\text { std. Dev. }\end{array}$ \\
\hline $\begin{array}{l}--1--1-\overline{0} \\
09 / 25 / 74 \\
10 / 25 / 73 \\
10 / 25 / 72 \\
10 / 01 / 71\end{array}$ & --- & $\begin{array}{l}0.3800 \mathrm{E}+00 \\
0.1100 \mathrm{E}-01 \\
0.7740 \mathrm{E}-01 \\
0.1410 \mathrm{E}+00\end{array}$ & $\begin{array}{l}0.000 \mathrm{E}+00 \\
0.230 \mathrm{E}-02 \\
0.000 \mathrm{E}+00 \\
0.000 \mathrm{E}+00\end{array}$ \\
\hline
\end{tabular}

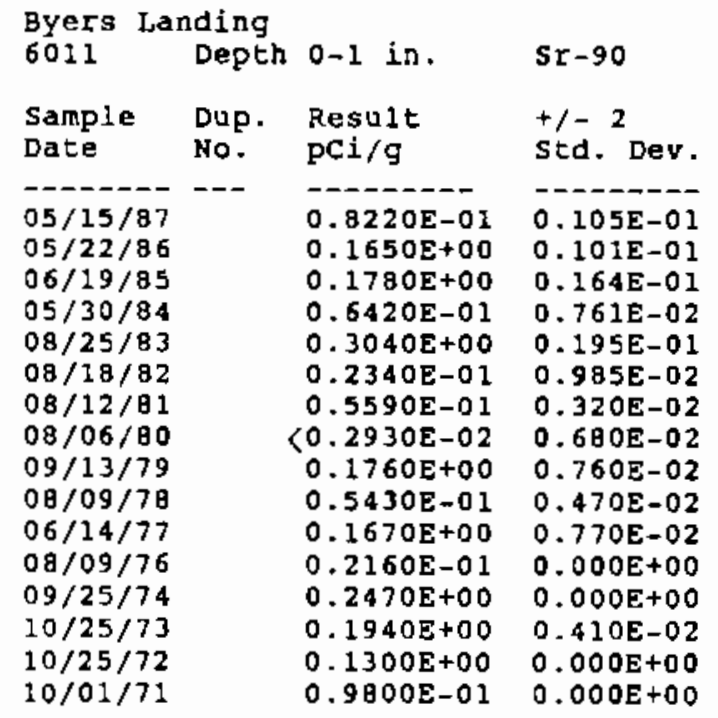

$\begin{array}{llll}\begin{array}{l}\text { Byers Landing } \\ 6034\end{array} & & \\ & \text { Depth } & 1-2 \text { in. } & \text { Sr-90 } \\ \text { Sample } & \text { Dup. } & \text { Result } & +/-2 \\ \text { Date } & \text { No. } & \text { pCi/g } & \text { Std. Dev. } \\ -10 / 25 / 72 & & 0.1770 E+00 & 0.000 E+00 \\ 10 / 01 / 71 & & 0.9800 E-01 & 0.000 E+00\end{array}$

Rlverview (Harris Farm)

6361 Depth $0-1$ in. Sr-90

Sample Dup, Result $+/-2$

Date No, pCi/g std. Dev.

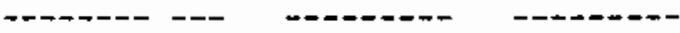

$05 / 15 / 87 \quad 0.1870 \mathrm{E}+00 \quad 0.135 \mathrm{E}-01$

$05 / 22 / 86 \quad 0.6110 \mathrm{E}-01 \quad 0.116 \mathrm{E}-01$

$06 / 19 / 85 \quad 0.7410 \mathrm{E}-01 \quad 0.118 \mathrm{AE}-01$

$05 / 30 / 84 \quad 0.3910 E-01 \quad 0.115 E-01$

$0 B / 18 / 83 \quad 0.8970 E+00 \quad 0.437 E-01$

$0 B / 1 \mathrm{~B} / 82 \quad 0.1150 \mathrm{E}+00 \quad 0.409 \mathrm{E}-01$

$08 / 12 / 81 \quad 0.2090 E+00 \quad 0.900 E-02$

$08 / 06 / 80<<0.1350 \mathrm{E}-02 \quad 0.680 \mathrm{E}-02$

$09 / 13 / 79 \quad 0.3740 E+00 \quad 0.110 \mathrm{E}-01$

$08 / 09 / 78 \quad 0.3540 E-01 \quad 0.380 \mathrm{E}-02$

$06 / 14 / 77 \quad 0.1740 E-01 \quad 0.340 E-02$

Riverview Control Plot 55

\begin{tabular}{|c|c|c|c|}
\hline 012 & Depth & $0-1$ in. & $S r-90$ \\
\hline $\begin{array}{l}\text { Sample } \\
\text { late }\end{array}$ & $\begin{array}{l}\text { Dup. } \\
\text { No. }\end{array}$ & $\begin{array}{l}\text { Result } \\
\text { pci/g }\end{array}$ & $\begin{array}{l}+/-2 \\
\text { std. Dev. }\end{array}$ \\
\hline $\begin{array}{l}9 / 25 / 74 \\
0 / 25 / 73 \\
0 / 25 / 72 \\
10 / 01 / 71\end{array}$ & $m--$ & $\begin{array}{l}0.3950 \mathrm{E}-01 \\
0.6420 \mathrm{E}-01 \\
0.22 \mathrm{~B} 0 \mathrm{E}+00 \\
0.3300 \mathrm{E}+00\end{array}$ & $\begin{array}{l}0.000 \mathrm{E}+00 \\
0.290 \mathrm{D}-02 \\
0.000 \mathrm{E}+00 \\
0.000 \mathrm{E}+00\end{array}$ \\
\hline
\end{tabular}

$\begin{array}{llll}\begin{array}{l}\text { Riverview Control Plot } 55 \\ \text { 6035 } \\ \text { Depth } 1-2 \text { in. }\end{array} & \text { Sr-90 } \\ & & & \\ \text { Sample } & \text { Dup. } & \text { Result } & +/-2 \\ \text { Date } & \text { No. } & \text { pCi/g } & \text { Std. Dev. } \\ -10 / 25 / 72 & & 0.1700 \mathrm{E}+00 & 0.000 \mathrm{E}+00 \\ 10 / 01 / 71 & & 0.1960 \mathrm{E}+00 & 0.000 \mathrm{E}+00\end{array}$


TABLE B.2. (contd)

\begin{tabular}{|c|c|c|c|}
\hline \multicolumn{3}{|c|}{ N Richland Control Plot 56} & $S z-90$ \\
\hline $\begin{array}{l}\text { Sample } \\
\text { Date }\end{array}$ & $\begin{array}{l}\text { Dup. } \\
\text { No. }\end{array}$ & $\begin{array}{l}\text { Regult } \\
\text { pCi/g }\end{array}$ & $\begin{array}{l}+/-2 \\
\text { std. Dev. }\end{array}$ \\
\hline $\begin{array}{l}-09 / 25 / 74 \\
09 / 25 / 73 \\
10 / 25 / 72 \\
09 / 30 / 71\end{array}$ & -+- & $\begin{array}{l}0.2610 \mathrm{E}+00 \\
0.1180 \mathrm{E}+00 \\
0.3180 \mathrm{E}+00 \\
0.2400 \mathrm{E}+00\end{array}$ & $\begin{array}{l}0.000 \mathrm{E}+00 \\
0.290 \mathrm{E}-02 \\
0.000 \mathrm{E}+00 \\
0.000 \mathrm{E}+00\end{array}$ \\
\hline
\end{tabular}

\begin{tabular}{llll}
$\begin{array}{l}\text { Benton City } \\
6023\end{array}$ & Depth & $1-2$ in. & sr-90 \\
& & & \\
Sample & Dup. & Regult & $+/-2$ \\
Date & No. & pCi/g & std. Dev. \\
\hline $10 / 25 / 72$ & & $0.2700 \mathrm{E}+00$ & $0.000 \mathrm{E}+00$ \\
$09 / 30 / 71$ & & $0.2170 \mathrm{E}+00$ & $0.000 \mathrm{E}+00$
\end{tabular}

N Richland Control Plot 56

\begin{tabular}{llll}
6036 & Depth $1-2$ in, & sz-90 \\
Sample & Dup. & Result & $+/-2$ \\
Date & No. & pCi/g & std, Dev. \\
\hline $10 / 25 / 72$ & & $0.1800 \mathrm{E}+00$ & $0.000 \mathrm{E}+00$ \\
$09 / 30 / 71$ & & $0.2400 \mathrm{E}+00$ & $0.000 \mathrm{E}+00$
\end{tabular}

\begin{tabular}{llll}
$\begin{array}{l}\text { Benton City (QC Dupl. A) } \\
0820\end{array}$ & septh $0-1$ in. & Sr-90 \\
Sample & Dup. Result & $+/-2$ \\
Date & No. & pCi $/ 9$ & std. Dev. \\
\hdashline $08 / 17 / 82$ & & $0.2170 \mathrm{E}+00$ & $0.108 \mathrm{E}+00$
\end{tabular}

NE Corner of Exxon site

$\begin{array}{llll}6280 & \text { Depth } 0-1 \text { in. } & \text { Sr-90 } \\ \text { Sample } & \text { Dup. } & \text { Regult } & +/-2 \\ \text { Date } & \text { No. } & \text { pCi/g } & \text { Std. Dev. } \\ -08 / 09 / 76 & & 0.2530 E+00 & 0.000 E+00 \\ 08 / 19 / 75 & & 0.5550 \mathrm{E}+00 & 0.000 \mathrm{E}+00\end{array}$

Benton City

\begin{tabular}{llll}
6000 & Depth $0-1$ in. & st-90 \\
Sample & Dup. & Result & $+/-2$ \\
Date & No. & pCi/g & std. Dev. \\
\hline $05 / 20 / 87$ & & $0.2400 E+00$ & $0.139 E-01$ \\
$05 / 23 / 86$ & & $0.2450 E+00$ & $0.101 E-01$ \\
$07 / 03 / 85$ & & $0.1180 E+00$ & $0.144 E-01$ \\
$06 / 06 / 84$ & $0.3590 E+00$ & $0.306 E-01$ \\
$08 / 23 / 83$ & $0.4180 E+00$ & $0.181 E-01$ \\
$08 / 16 / 82$ & $0.2120 E+00$ & $0.298 E-01$ \\
$08 / 13 / 81$ & $0.2950 E+00$ & $0.320 E-02$ \\
$08 / 04 / 80$ & $0.280 E+00$ & $0.510 E-02$ \\
$09 / 12 / 79$ & $0.3360 E+00$ & $0.990 E-02$ \\
$08 / 08 / 78$ & $0.3200 E-01$ & $0.360 E-02$ \\
$06 / 14 / 77$ & $0.2500 E-01$ & $0.360 E-02$ \\
$09 / 25 / 74$ & $0.7770 E+00$ & $0.000 E+00$ \\
$10 / 25 / 73$ & $0.1230 E+00$ & $0.340 E-02$ \\
$10 / 25 / 72$ & $0.3790 E+00$ & $0.000 E+00$ \\
$09 / 30 / 71$ & $0.4500 E+00$ & $0.000 E+00$
\end{tabular}

Benton City (QC Dupl. B)

0821 Depth $0-1$ in.

Sample Dup. Reault $+/-2$

Date No. pCi/g std. Dev.

$\begin{array}{llll}08 / 17 / 82 & - & 0.6970 \mathrm{E}-02 & 0.177 \mathrm{E}-02\end{array}$

\section{$08 / 17 / 82$}

Sunnyside

\begin{tabular}{llll}
6357 & Depth & $0-1 \mathrm{in}$. & Sr-90 \\
Sample & Dup. & Regult & $+/-2$ \\
Date & No. & pCi/g & std. Dev. \\
\hline $05 / 21 / 87$ & 1 & $0.2540 \mathrm{E}+00$ & $0.221 \mathrm{E}-01$ \\
$06 / 03 / 86$ & & $0.5220 \mathrm{E}-01$ & $0.776 \mathrm{E}-02$ \\
$06 / 17 / 85$ & & $0.2620 \mathrm{E}+00$ & $0.197 \mathrm{E}-01$ \\
$05 / 22 / 84$ & & $0.3100 \mathrm{E}+00$ & $0.294 \mathrm{E}-01$ \\
$08 / 23 / 83$ & & $0.1610 \mathrm{E}+01$ & $0.397 \mathrm{E}-01$ \\
$08 / 17 / 82$ & & $0.1200 \mathrm{E}+00$ & $0.315 \mathrm{E}-01$ \\
$08 / 13 / 81$ & & $0.1570 \mathrm{E}+00$ & $0.160 \mathrm{~B}-01$ \\
$08 / 04 / 80$ & & $0.1900 \mathrm{E}+00$ & $0.210 \mathrm{E}-01$ \\
$09 / 12 / 79$ & & $0.2450 \mathrm{E}+00$ & $0.990 \mathrm{E}-02$ \\
$08 / 08 / 78$ & & $0.1370 \mathrm{E}-01$ & $0.310 \mathrm{E}-02$ \\
$06 / 14 / 77$ & & $0.3330 \mathrm{E}-01$ & $0.430 \mathrm{E}-02$
\end{tabular}

\begin{tabular}{llll}
$\begin{array}{l}\text { Yakima } \\
0512\end{array}$ & Depth & $0-1$ in. & Sr-90 \\
& & & \\
Sample & Dup. & Result & $+/-2$ \\
Date & No. & pCi/g & Std. Dev. \\
\hdashline $05 / 21 / 87$ & 1 & $0.4610 \mathrm{E}-01$ & $0.772 \mathrm{E}-02$ \\
$05 / 21 / 87$ & & $0.6790 \mathrm{E}-01$ & $0.87 \mathrm{E}-02$ \\
$06 / 03 / 86$ & & $0.3890 \mathrm{E}-01$ & $0.661 \mathrm{E}-02$
\end{tabular}


TABLE B.2. (contd)

\begin{tabular}{llll} 
Moses Lake & & \\
0500 & Depth & $0-1$ in. & Sr-90 \\
Sample & Dup. & Result & $+/-2$ \\
Date & No. & pCi/g & std. Dev. \\
\hline $05 / 08 / 87$ & 1 & $0.6950 \mathrm{E}-01$ & $0.103 \mathrm{E}-01$ \\
$05 / 08 / 87$ & & $0.5700 \mathrm{E}-01$ & $0.100 \mathrm{E}-01$ \\
$05 / 23 / 86$ & & $0.7570 \mathrm{E}-01$ & $0.600 \mathrm{E}-02$
\end{tabular}

\begin{tabular}{llll}
$\begin{array}{l}\text { MeNary } \\
\text { 0504 }\end{array}$ & Dam & & \\
& Depth & $0-1 \mathrm{in}$. & Sr-90 \\
Sample & Dup. & Regult & $+/-2$ \\
Date & No. & pCi/g & std. Dev. \\
\hline $05 / 29 / 87$ & & $0.9100 \mathrm{E}-01$ & $0.95 \mathrm{EE}-02$ \\
$05 / 29 / 87$ & 1 & $0.5440 \mathrm{E}-01$ & $0.738 \mathrm{E}-02$ \\
$05 / 02 / 86$ & & $0.1750 \mathrm{E}+00$ & $0.022 \mathrm{E}-02$ \\
$11 / 15 / 85$ & & $0.2870 \mathrm{E}+00$ & $0.188 \mathrm{E}-01$
\end{tabular}

\begin{tabular}{llll}
$\begin{array}{l}\text { Othello } \\
\text { 0505 }\end{array}$ & Depth $0-1$ in. & Sr-90 \\
Sample & Dup. & Regult & $+/-2$ \\
Date & No. & pCi/g & Std. Dev. \\
\hline $05 / 26 / 87$ & 1 & $0.1310 \mathrm{E}+00$ & $0.105 \mathrm{E}-01$ \\
$05 / 26 / 87$ & & $0.1730 \mathrm{E}+00$ & $0.123 \mathrm{E}-01$ \\
$05 / 02 / 86$ & & $0.2720 \mathrm{E}+00$ & $0.236 \mathrm{E}-01$
\end{tabular}

\begin{tabular}{llll}
$\begin{array}{l}\text { Connell } \\
0503\end{array}$ & Depth $0-1$ in. & sr-90 \\
Sample & Dup. & Reeult & $+/-2$ \\
Date & No. & pCi $/ 9$ & std. Dev. \\
\hline $05 / 26 / 87$ & 1 & $0.8950 \mathrm{E}-01$ & $0.921 \mathrm{E}-02$ \\
$05 / 26 / 87$ & & $0.1520 \mathrm{E}+00$ & $0.113 \mathrm{E}-01$ \\
$05 / 02 / 86$ & & $0.3170 \mathrm{E}+00$ & $0.163 \mathrm{E}-01$
\end{tabular}

\begin{tabular}{llll}
$\begin{array}{l}\text { Washtucna } \\
0501\end{array}$ & Depth & $0-1 \mathrm{in.}$ & $5 \mathrm{r}-90$ \\
Sample & Dup. & Result & $+/-2$ \\
Date & No. & $\mathrm{pCi} / \mathrm{g}$ & Std. Dev. \\
\hline $05 / 08 / 87$ & 1 & $0.1490 \mathrm{E}+00$ & $0.129 \mathrm{E}-01$ \\
$05 / 08 / 87$ & & $0.8040 \mathrm{E}-01$ & $0.106 \mathrm{E}-01$ \\
$05 / 23 / 86$ & & $0.4010 \mathrm{E}+00$ & $0.185 \mathrm{E}-01$
\end{tabular}

\begin{tabular}{llll}
$\begin{array}{l}\text { Walla Walla } \\
0502\end{array}$ & $\begin{array}{l}\text { Depth } \\
0-1 \text { in. }\end{array}$ & Sr-90 \\
Sample & Dup. & Result & $+/-2$ \\
Date & No. & pCi/g & std. Dev. \\
\hline $05 / 29 / 97$ & & $0.1940 E-01$ & $0.533 E-02$ \\
$05 / 29 / 97$ & 1 & $0.2190 E-01$ & $0.605 E-02$ \\
$05 / 02 / 96$ & & $0.1380 E+00$ & $0.789 E-02$ \\
$11 / 15 / 85$ & & $0.31108+00$ & $0.147 E-01$
\end{tabular}


TABLE B.3. Cesium-137 Concentrations in soil at offsite Locations

\begin{tabular}{llll} 
Wahluke Slope No. 2 & \\
6007 & Depth $0-1$ in. & Cs -137 \\
Sample & Dup, & Result & $+/ 2$ \\
Date & No. & pCi/g & std. Dev. \\
\hline $05 / 26 / 87$ & & $0.1590 \mathrm{E}+00$ & $0.316 \mathrm{E}-01$ \\
$05 / 02 / 86$ & $0.3030 \mathrm{E}+00$ & $0.330 \mathrm{E}-01$ \\
$06 / 12 / 85$ & $0.4740 \mathrm{E}+00$ & $0.472 \mathrm{E}-01$ \\
$05 / 31 / 84$ & $0.2800 \mathrm{E}+00$ & $0.284 \mathrm{E}-01$ \\
$08 / 26 / 83$ & $0.2520 \mathrm{E}+00$ & $0.291 \mathrm{E}-01$ \\
$08 / 30 / 82$ & $0.3420 \mathrm{E}+00$ & $0.687 \mathrm{E}-01$ \\
$08 / 11 / 81$ & $0.1010 \mathrm{E}+00$ & $0.180 \mathrm{E}-01$ \\
$08 / 06 / 80$ & $0.3490 \mathrm{E}+00$ & $0.520 \mathrm{E}-01$ \\
$09 / 13 / 79$ & $0.4630 \mathrm{E}+00$ & $0.640 \mathrm{E}-01$ \\
$08 / 09 / 78$ & $0.3270 \mathrm{E}+00$ & $0.550 \mathrm{E}-01$ \\
$06 / 14 / 77$ & $0.1770 \mathrm{E}+00$ & $0.430 \mathrm{E}-01$ \\
$08 / 09 / 76$ & $0.2880 \mathrm{E}+00$ & $0.000 \mathrm{E}+00$ \\
$08 / 19 / 75$ & $0.3130 \mathrm{E}+00$ & $0.000 \mathrm{E}+00$ \\
$09 / 23 / 74$ & $0.2290 \mathrm{E}+00$ & $0.000 \mathrm{E}+00$ \\
$10 / 25 / 73$ & $0.1010 \mathrm{E}+01$ & $0.000 \mathrm{E}+00$ \\
$10 / 25 / 72$ & $0.7240 \mathrm{E}+00$ & $0.000 \mathrm{E}+00$ \\
$09 / 29 / 71$ & $0.4680 \mathrm{E}+00$ & $0.000 \mathrm{E}+00$
\end{tabular}

\begin{tabular}{|c|c|c|c|}
\hline $\begin{array}{l}\text { Wahluke } \\
6030\end{array}$ & $\begin{array}{l}\text { Slope } 1 \\
\text { Depth }\end{array}$ & No. ${ }^{2}$ in. & $\mathrm{Cs}-137$ \\
\hline $\begin{array}{l}\text { Sample } \\
\text { Date }\end{array}$ & $\begin{array}{l}\text { Dup. } \\
\text { No. }\end{array}$ & $\begin{array}{l}\text { Result } \\
\text { pci/g }\end{array}$ & $\begin{array}{l}+/-2 \\
\text { std. Dev. }\end{array}$ \\
\hline $\begin{array}{l}10 / 25 / 72 \\
09 / 29 / 71\end{array}$ & & $\begin{array}{l}0.1580 \mathrm{E}+00 \\
0.2560 \mathrm{E}+00\end{array}$ & $\begin{array}{l}0.000 E+00 \\
0.000 E+00\end{array}$ \\
\hline
\end{tabular}

Berg Ranch

\begin{tabular}{llll}
6008 & Depth & $0-1 \mathrm{in}$. & Cs-137 \\
Sample & Dup. & Regult & $+/-2$ \\
Date & No. & pCi/g & std. Dev. \\
\hline $05 / 26 / 87$ & & $0.3100 \mathrm{E}+00$ & $0.354 \mathrm{E}-01$ \\
$05 / 02 / 86$ & & $0.6020 \mathrm{E}+00$ & $0.454 \mathrm{E}-01$ \\
$06 / 26 / 85$ & & $0.5790 \mathrm{E}+00$ & $0.521 \mathrm{E}-01$ \\
$05 / 30 / 84$ & & $0.4940 \mathrm{E}+00$ & $0.457 \mathrm{E}-01$ \\
$08 / 26 / 83$ & & $0.6080 \mathrm{E}+00$ & $0.453 \mathrm{E}-01$ \\
$08 / 20 / 82$ & & $0.8340 \mathrm{E}+00$ & $0.512 \mathrm{E}-01$ \\
$08 / 11 / 81$ & 1 & $0.4850 \mathrm{E}+00$ & $0.380 \mathrm{E}-01$ \\
$08 / 13 / 81$ & & $0.3960 \mathrm{E}+00$ & $0.240 \mathrm{E}-01$ \\
$08 / 06 / 80$ & & $0.5820 \mathrm{E}+00$ & $0.640 \mathrm{E}-01$ \\
$09 / 13 / 79$ & & $0.8630 \mathrm{E}+00$ & $0.850 \mathrm{E}-01$ \\
$08 / 09 / 78$ & & $0.5480 \mathrm{E}+00$ & $0.680 \mathrm{E}-01$ \\
$06 / 14 / 77$ & & $0.1380 \mathrm{E}+01$ & $0.110 \mathrm{E}+00$ \\
$08 / 09 / 76$ & & $0.2950 \mathrm{E}+00$ & $0.000 \mathrm{E}+00$ \\
$00 / 19 / 75$ & & $0.3220 \mathrm{E}+00$ & $0.000 \mathrm{D}+00$ \\
$09 / 23 / 74$ & & $0.4510 \mathrm{E}+00$ & $0.000 \mathrm{E}+00$ \\
$10 / 25 / 73$ & & $0.0220 \mathrm{E}+00$ & $0.000 \mathrm{E}+00$ \\
$10 / 25 / 72$ & & $0.2260 \mathrm{E}+00$ & $0.000 \mathrm{E}+00$ \\
$09 / 30 / 71$ & & $0.5690 \mathrm{E}+00$ & $0.000 \mathrm{E}+00$
\end{tabular}

Berg Ranch

\begin{tabular}{llll}
6031 & Depth $1-2$ in. & Cs-137 \\
Sample & Dup. & Result & $+/-2$ \\
Date & No. & pCi/g & Std. Dev. \\
\hline $10 / 25 / 72$ & & $0.1200 E-01$ & $0.000 \mathrm{E}+00$ \\
$09 / 29 / 71$ & & $<0.0000 \mathrm{E}+00$ & $0.000 \mathrm{E}+00$
\end{tabular}

Cook Brog. Dairy Farm

$\begin{array}{llll}6219 & \text { Deptb 0-1 in. } & \text { Co-137 } \\ \text { Sample } & \text { Dup. Result } & +/-2 \\ \text { Date } & \text { No. } & \text { pci/g } & \text { Std. Dev. } \\ -09 / 25 / 74 & & 0.74008-01 & 0.000 \mathrm{E}+00\end{array}$

Ringold

6009 Depth $0-1$ in. Cs-137

Sample Dup. Result $+/-2$

Date No. pCi/g std. Dev.

05/15/07 -

$05 / 22 / 86 \quad 0.3980 \mathrm{E}+00 \quad 0.428 \mathrm{E}-01$

$07 / 0 \mathrm{~B} / 85 \quad 0.1120 \mathrm{E}+01 \quad 0.45 \mathrm{EE}-01$

$05 / 30 / 84 \quad 0.4440 \mathrm{E}+00 \quad 0.436 \mathrm{E}-01$

$08 / 18 / 83 \quad 0.1560 E+01 \quad 0.805 E-01$

$08 / 20 / 82 \quad 0.82608+00 \quad 0.551 E-01$

$08 / 12 / 81 \quad 0.9380 \mathrm{E}+00 \quad 0.480 \mathrm{E}-01$

$08 / 06 / 80 \quad 0.6780 \mathrm{E}+00 \quad 0.690 \mathrm{E}-01$

$09 / 13 / 79 \quad 0.6920 \mathrm{E}+00 \quad 0.760 \mathrm{E}-01$

$08 / 09 / 7 \mathrm{~B} \quad 0.9670 \mathrm{E}+00 \quad 0.870 \mathrm{E}-01$

$06 / 14 / 77 \quad 0.2070 E+00 \quad 0.460 E-01$

$08 / 09 / 76 \quad 0.4340 E+00 \quad 0.000 E+00$

$08 / 19 / 75 \quad 0.1710 \mathrm{E}+00 \quad 0.000 \mathrm{E}+00$

$10 / 25 / 73 \quad<0.0000 E+00 \quad 0.000 E+00$

$10 / 25 / 72 \quad 0.4000 \mathrm{E}+00 \quad 0.000 \mathrm{E}+00$

$10 / 01 / 71 \quad 0.4080 \mathrm{E}+00 \quad 0.000 \mathrm{E}+00$

\begin{tabular}{llll}
$\begin{array}{l}\text { Ringold } \\
6032\end{array}$ & Depth & $1-2$ in. & Cs-137 \\
Sample & Dup, & Regult & $+/{ }^{2}$ \\
Date & No. & pCi/g & Std. Dev. \\
\hline $10 / 25 / 72$ & & $0.4800 E-01$ & $0.000 \mathrm{E}+00$ \\
$10 / 01 / 71$ & & $0.3230 \mathrm{E}-01$ & $0.000 \mathrm{O}+00$
\end{tabular}


TABLE B. 3. (contd)

\begin{tabular}{llll}
$\begin{array}{l}\text { W End of Fir Road } \\
6360\end{array}$ & Depth $0-1$ In. & Cs-137 \\
& & & \\
Sample & Dup. & Result & $+/-2$ \\
Date & No. & PCi/g & Std. Dev. \\
\hline $05 / 15 / 87$ & & $0.2270 E+00$ & $0.333 E-01$ \\
$05 / 22 / 86$ & $0.2540 E+00$ & $0.302 E-01$ \\
$07 / 03 / 85$ & $0.1410 E+00$ & $0.246 E-01$ \\
$05 / 31 / 84$ & $0.1160 E+00$ & $0.308 E-01$ \\
$08 / 18 / 83$ & $0.2500 E+00$ & $0.345 E-01$ \\
$08 / 20 / 82$ & $0.3470 E+00$ & $0.546 E-01$ \\
$08 / 12 / 81$ & $0.1470 E+00$ & $0.200 E-01$ \\
$08 / 06 / 80$ & $0.1850 E+00$ & $0.400 E-01$ \\
$09 / 13 / 79$ & $0.5710 E+00$ & $0.690 E-01$ \\
$08 / 09 / 78$ & $0.3920 E+00$ & $0.590 E-01$ \\
$06 / 14 / 77$ & $0.3990 E+00$ & $0.600 E-01$
\end{tabular}

Baxter Subatation

\begin{tabular}{llll}
6220 & Depth $0-1$ in. & Cs-137 \\
Sample & Dup. & Result & $+/-2$ \\
Date & No. & pCi/g & std. Dev. \\
\hline $06 / 14 / 77$ & & $0.1930 E+00$ & $0.450 E-01$ \\
$09 / 25 / 74$ & & $0.2570 \mathrm{E}+00$ & $0.000 \mathrm{E}+00$
\end{tabular}

\begin{tabular}{|c|c|c|c|}
\hline $\begin{array}{l}\text { Pettett } \\
6359\end{array}$ & $\begin{array}{l}\text { Dairy } \\
\text { Depth }\end{array}$ & $\begin{array}{c}\text { Farm } \\
0-1 \text { in. }\end{array}$ & $\mathrm{Ca}-137$ \\
\hline $\begin{array}{l}\text { Sample } \\
\text { Date }\end{array}$ & $\begin{array}{l}\text { Dup. } \\
\text { No. }\end{array}$ & $\begin{array}{l}\text { Result } \\
\text { pCi/g }\end{array}$ & $\begin{array}{l}+/-2 \\
\text { std. Dev. }\end{array}$ \\
\hline $06 / 14 / 77$ & $=--$ & $0.2920 \mathrm{E}+00$ & $0.530 \mathrm{E}-01$ \\
\hline
\end{tabular}

Taylor Flatg No. 1

\begin{tabular}{llll}
6420 & \multicolumn{2}{l}{ Depth $0-1$ in. } & Cs-137 \\
Sample & Dup. & Result & $+/-2$ \\
Date & No. & pCi/g & Std. Dev. \\
\hline $08 / 18 / 82$ & & $<0.2530 E-01$ & $0.540 E-01$ \\
$08 / 14 / 81$ & & $0.7870 E+00$ & $0.420 E-01$ \\
$01 / 22 / 81$ & & $0.9890 E-01$ & $0.190 E-01$ \\
$08 / 06 / 80$ & & $0.8610 E-01$ & $0.360 E-01$ \\
$09 / 13 / 79$ & & $0.1270 E+00$ & $0.370 E-01$ \\
$08 / 10 / 78$ & & $0.2340 E+00$ & $0.480 E-01$
\end{tabular}

Taylor Flats No, 2

6421 Depth $0-1$ in. $\mathrm{Cs}-137$

Sample Dup. Regult $+/-2$

Date No. pCi/g std. Dev.

$05 / 15 / 87-0.6040 \mathrm{E}+00 \quad 0.548 \mathrm{E}-01$

$05 / 22 / 86 \quad 0.1190 E+01 \quad 0.132 E+00$

$07 / 08 / 85 \quad 0.8540 \mathrm{E}-01 \quad 0.281 \mathrm{E}-01$

$05 / 30 / 84 \quad 0.8420 E-01 \quad 0.310 E-01$

$08 / 26 / 83 \quad 0.2180 E+01 \quad 0.702 E-01$

$08 / 20 / 82 \quad 0.6050 E+00 \quad 0.488 E-0 I$

$11 / 03 / 81 \quad 0.3720 \mathrm{E}+00 \quad 0.180 \mathrm{E}-01$

$11 / 03 / 812 \quad 0.4050 E+00 \quad 0.320 E-01$

$11 / 03 / 81 \quad 1 \quad 0.3160 E+00 \quad 0.290 E-01$

$08 / 14 / 81 \quad 0.1070 E+01 \quad 0.510 E-01$

$01 / 22 / 81 \quad 0.2700 E+00 \quad 0.210 E-01$

$08 / 06 / 80 \quad 0.1400 \mathrm{E}+01 \quad 0.110 \mathrm{E}+00$

$09 / 13 / 79 \quad 0.1600 E+01 \quad 0.110 E+00$

$08 / 10 / 78 \quad 0.9120 E-01 \quad 0.340 E-01$

\begin{tabular}{llll}
$\begin{array}{l}\text { Sagemore } \\
6358\end{array}$ & $\begin{array}{l}\text { Farma } \\
\text { Depth }\end{array}$ 0-1 in. & Cg-137 \\
Sample & Dup. & Regult & $+/-2$ \\
Date & No. & pCi/g & Std. Dev. \\
\hline $05 / 15 / 87$ & & $0.1170 E+00$ & $0.241 E-01$ \\
$05 / 22 / 86$ & & $0.3210 E+00$ & $0.393 E-01$ \\
$07 / 08 / 85$ & & $0.1030 E+00$ & $0.225 E-01$ \\
$05 / 30 / 84$ & & $0.1020 E+01$ & $0.642 E-01$ \\
$08 / 25 / 83$ & & $0.1410 E+00$ & $0.264 E-01$ \\
$08 / 18 / 82$ & & $0.5860 E-01$ & $0.372 E-01$ \\
$08 / 12 / 81$ & 1 & $0.4490 E-01$ & $0.160 E-01$ \\
$08 / 12 / 81$ & & $0.5890 E-01$ & $0.150 E-01$ \\
$08 / 06 / 80$ & & $0.5510 E+00$ & $0.630 E-01$ \\
$09 / 13 / 79$ & & $0.8950 E-01$ & $0.300 E-01$ \\
$08 / 09 / 78$ & & $0.1180 E+00$ & $0.360 E-01$ \\
$06 / 14 / 77$ & & $0.7060 E-01$ & $0.320 E-01$
\end{tabular}

\begin{tabular}{|c|c|c|c|}
\hline $\begin{array}{l}\text { agemore } \\
\text { ois }\end{array}$ & $\begin{array}{l}\text { Farms } \\
\text { Depth }\end{array}$ & $\begin{array}{l}\text { (QC Dupl. } \\
0-1 \text { in. }\end{array}$ & $\mathrm{Cs}-137$ \\
\hline $\begin{array}{l}\text { ample } \\
\text { ate }\end{array}$ & $\begin{array}{l}\text { Dup. } \\
\text { No. }\end{array}$ & $\begin{array}{l}\text { Regult } \\
\mathrm{pCi} / \mathrm{g}\end{array}$ & $\begin{array}{l}+/{ }^{2} \\
\text { std. Dev. }\end{array}$ \\
\hline $\begin{array}{l}5 / 15 / 87 \\
5 / 22 / 86 \\
7 / 08 / 85 \\
5 / 30 / 84 \\
8 / 25 / 83 \\
8 / 18 / 82\end{array}$ & -- & $\begin{array}{r}0.2810 \mathrm{E}+00 \\
0.2440 \mathrm{E}+00 \\
\angle 0.3310 \mathrm{E}-02 \\
0.1040 \mathrm{E}+01 \\
0.9150 \mathrm{E}-01 \\
0.4040 \mathrm{E}+00\end{array}$ & $\begin{array}{l}0.315 E-01 \\
0.348 \mathrm{E}-01 \\
0.360 \mathrm{E}-01 \\
0.662 \mathrm{E}-01 \\
0.243 \mathrm{E}-01 \\
0.640 \mathrm{E}-01\end{array}$ \\
\hline
\end{tabular}


TABLE B.3. (contd)

\begin{tabular}{|c|c|c|c|}
\hline $\begin{array}{l}\text { Sagemore } \\
0819\end{array}$ & $\begin{array}{l}\text { Farms } \\
\text { Depth }\end{array}$ & $\begin{array}{l}\text { COC Dupl. E } \\
\text { O-1 in. }\end{array}$ & $\mathrm{Cs}-137$ \\
\hline $\begin{array}{l}\text { Sample } \\
\text { Date }\end{array}$ & $\begin{array}{l}\text { Dup. } \\
\text { No. }\end{array}$ & $\begin{array}{l}\text { Result } \\
\text { pCi/g }\end{array}$ & $\begin{array}{l}+/-2 \\
\text { std. Dev. }\end{array}$ \\
\hline $\begin{array}{l}-05 / 15 / 87 \\
05 / 22 / 86 \\
07 / 08 / 85 \\
05 / 30 / 84 \\
08 / 25 / 83 \\
08 / 18 / 82\end{array}$ & --- & $\begin{array}{l}--1-\overline{0} \\
0.3290 \mathrm{E}+00 \\
0.2940 \mathrm{E}+00 \\
0.7160 \mathrm{E}-01 \\
0.1110 \mathrm{E}+01 \\
0.1340 \mathrm{E}+00 \\
0.18 \mathrm{~B} 0 \mathrm{E}+00\end{array}$ & $\begin{array}{l}0.364 \mathrm{E}-01 \\
0.346 \mathrm{E}-01 \\
0.207 \mathrm{E}-01 \\
0.559 \mathrm{E}-01 \\
0.265 \mathrm{E}-01 \\
0.274 \mathrm{E}-01\end{array}$ \\
\hline
\end{tabular}

Byers Landing Pump House

6010 Depth $0-1$ in.

Sample Dup. Result $\quad+/-2$

Date No. pCi/g std. Dev.

-

$10 / 25 / 73 \quad 0.1420 E+00 \quad 0.000 E+00$

$10 / 25 / 72 \quad 0.4240 E+00 \quad 0.000 E+00$

$10 / 01 / 71 \quad 0.6030 E+00 \quad 0.000 E+00$

Byers Landing Pump Houge

6033 Depth 1-2 in.

Cs -137

Sample Dup. Result $+/-2$

Date No. pCilg std. Dev,

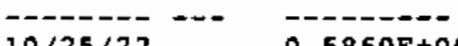

$10 / 01 / 71 \quad 0.1750 \mathrm{E}+00$

$0.000 \mathrm{E}+00$

$0.000 \mathrm{E}+00$

\begin{tabular}{llll}
$\begin{array}{l}\text { Byers Landing } \\
6011\end{array}$ & Depth & $0-1$ in. & Ce-137 \\
Sample & Dup. & Regult & $+/-2$ \\
Date & No. & pCi/g & Std. Dev. \\
\hline $05 / 15 / 87$ & & $0.2270 E+00$ & $0.304 E-01$ \\
$05 / 22 / 86$ & $0.5040 E+00$ & $0.36 \theta E-01$ \\
$06 / 19 / 85$ & $0.1930 E+00$ & $0.347 E-01$ \\
$05 / 30 / 84$ & $0.2020 E+00$ & $0.305 E-01$ \\
$08 / 25 / 83$ & $0.5870 E+00$ & $0.460 E-01$ \\
$08 / 18 / 82$ & $0.2790 E+00$ & $0.745 E-01$ \\
$08 / 12 / 81$ & $0.2280 E+00$ & $0.240 E-01$ \\
$08 / 06 / 80$ & $0.3410 E+00$ & $0.490 E-01$ \\
$09 / 13 / 79$ & $0.9370 E+00$ & $0.850 E-01$ \\
$08 / 09 / 78$ & $0.1100 E+01$ & $0.950 E-01$ \\
$06 / 14 / 77$ & $0.4240 E+00$ & $0.620 E-01$ \\
$08 / 09 / 76$ & $0.2010 E+00$ & $0.000 E+00$ \\
$09 / 25 / 74$ & $0.6940 E+00$ & $0.000 E+00$ \\
$10 / 25 / 73$ & $0.7650 E+00$ & $0.000 E+00$ \\
$10 / 25 / 72$ & $0.8360 E+00$ & $0.000 E+00$ \\
$10 / 01 / 71$ & $0.1270 E+01$ & $0.000 E+00$
\end{tabular}

\begin{tabular}{llll}
$\begin{array}{l}\text { Byerg Landing } \\
6034\end{array}$ & Depth & & \\
& & & \\
& & in. & Cs-137 \\
Sample & Dup. & Result & $+/-2$ \\
Date & No. & pCi/g & Std. Dev. \\
\hline $10 / 25 / 72$ & & $0.0810 \mathrm{E}+00$ & $0.000 \mathrm{E}+00$ \\
$10 / 01 / 71$ & & $0.2740 \mathrm{E}+00$ & $0.000 \mathrm{E}+00$
\end{tabular}

Riverview (Harrig Farm)

6361 Depth 0-1 in.

$\mathrm{Cs}-137$

Sample Dup. Regult $t /-2$

Date No. pCi/g std. Dev.

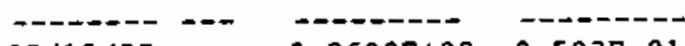

$05 / 15 / 97 \quad 0.8600 \mathrm{E}+00 \quad 0.503 \mathrm{E}-01$

$05 / 22 / 86 \quad 0.1680 \mathrm{E}+00 \quad 0.358 \mathrm{E}-01$

$06 / 19 / 85 \quad 0.2080 E+00 \quad 0.260 E-01$

$05 / 30 / 84 \quad 0.7710 E-01 \quad 0.205 E-01$

$08 / 18 / 83 \quad 0.1160 \mathrm{E}+01 \quad 0.653 \mathrm{E}-01$

$0 B / 18 / 82 \quad 0.4890 E+00 \quad 0.69 \theta E-01$

$08 / 12 / 81 \quad 0.4630 \mathrm{E}+00 \quad 0.330 \mathrm{E}-01$

$08 / 06 / 80 \quad 0.1090 \mathrm{E}+01 \quad 0.050 \mathrm{E}-01$

$09 / 13 / 79 \quad 0.3520 E+00 \quad 0.570 E-01$

$00 / 09 / 78 \quad 0.5010 \mathrm{E}+00 \quad 0.660 \mathrm{E}-01$

$06 / 14 / 77 \quad 0.16508+00 \quad 0.420 \mathrm{E}-01$

Riverview Control Plot 55

6012 Depth $0-1$ in. Cs-137

Sample Dup. Result $+/-2$

Date No. pCi/g std. Dev.

$09 / 25 / 74=0.58208-01 \quad 0.000 E+00$

$10 / 25 / 73 \quad 0.0380 E+00 \quad 0.000 E+00$

$10 / 25 / 72 \quad 0.5460 E+00 \quad 0.000 E+00$

$10 / 01 / 71 \quad 0.7430 \mathrm{E}+00 \quad 0.000 \mathrm{E}+00$

Riverview Control Plot 55

6035 Depth 1-2 in. Cs-137

Sample Dup. Result $+/-2$

Date No. pCi/g std. Dev.

$10 / 25 / 72-0.4540 \mathrm{E}+00 \quad 0.000 \mathrm{E}+00$

$10 / 01 / 71 \quad 0.2990 \mathrm{E}+00 \quad 0.000 \mathrm{E}+00$ 
TABLE B. 3. (contd)

N Richland Control Plot 56

$\begin{array}{llll}6013 & \text { Depth } 0-1 \text { in. } & \text { Cs-137. } \\ \text { Sample } & \text { Dup. } & \text { Result } & +/-2 \\ \text { Date } & \text { No. } & \text { pCi/g } & \text { std. Dev. } \\ -09 / 25 / 74 & -- & --470 \mathrm{E}+00 & 0.000 \mathrm{E}+00 \\ 09 / 25 / 73 & & 0.8380 \mathrm{E}+00 & 0.000 \mathrm{E}+00 \\ 10 / 25 / 72 & & 0.7530 \mathrm{E}+00 & 0.000 \mathrm{E}+00 \\ 09 / 30 / 71 & & 0.4180 \mathrm{E}+00 & 0.000 \mathrm{E}+00\end{array}$

N Richland Control Plot 56

\begin{tabular}{llll}
6036 & Depth $1-2$ in. & Cs-137 \\
& & & \\
Sample & Dup. & Result & $+/-2$ \\
Date & No. & pCi/g & Std. Dey. \\
\hline $10 / 25 / 72$ & & $0.3590 \mathrm{E}+00$ & $0.000 \mathrm{E}+00$ \\
$09 / 30 / 71$ & & $0.2590 \mathrm{E}+00$ & $0.000 \mathrm{E}+00$
\end{tabular}

NE Corner of Exxon site

6280 Depth $0-1$ in.

\begin{tabular}{|c|c|c|}
\hline $\begin{array}{l}\text { Sample } \\
\text { Date }\end{array}$ & $\begin{array}{l}\text { Dup. } \\
\text { No. }\end{array}$ & $\begin{array}{l}\text { Result } \\
\text { pCi/g }\end{array}$ \\
\hline $8 / 09 / 76$ & & 0.92 \\
\hline
\end{tabular}

Benton City

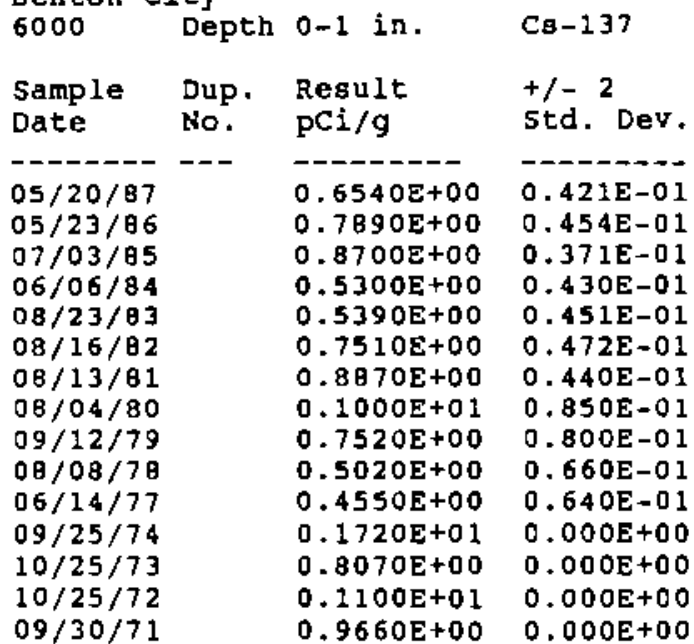

$\mathrm{C}=137$

$+1-2$

std. Dev.

$0.000 \mathrm{E}+00$

$0.000 \mathrm{E}+00$
Benton City

6023 Depth 1-2 in. Cg-137

Sample Dup. Result $+/-2$

Date No. $\mathrm{pCi} / \mathrm{g}$ std. Dev.

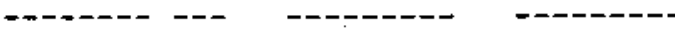

$10 / 25 / 72 \quad 0.84308+00 \quad 0.000 E+00$

$09 / 30 / 71 \quad 0.6010 \mathrm{E}-02 \quad 0.000 \mathrm{E}+00$

Benton City (QC Dupl. A)

0920 Depth $0-1$ in. Cs-137

\begin{tabular}{|c|c|c|c|}
\hline $\begin{array}{l}\text { Sample } \\
\text { Date }\end{array}$ & $\begin{array}{l}\text { Dup. } \\
\text { No. }\end{array}$ & $\begin{array}{l}\text { Result } \\
\text { pCi/g }\end{array}$ & $\begin{array}{l}+/-2 \\
\text { std. Dev. }\end{array}$ \\
\hline $8 / 17 / 82$ & $=-$ & $0.8090 \mathrm{E}+00$ & 0.934 \\
\hline
\end{tabular}

Sunnyside

\begin{tabular}{llll}
6357 & Depth & $0-1$ in. & Cs-137 \\
Sample & Dup. & Result & $+/-2$ \\
Date & No. & pCi/g & Std. Dev. \\
\hline $05 / 21 / 87$ & 1 & $0.2870 \mathrm{E}+00$ & $0.364 \mathrm{E}-01$ \\
$06 / 03 / 86$ & & $0.9710 \mathrm{E}-01$ & $0.263 \mathrm{E}-01$ \\
$06 / 17 / 85$ & & $0.2910 \mathrm{E}+00$ & $0.357 \mathrm{E}-01$ \\
$05 / 22 / 84$ & & $0.1520 \mathrm{E}+01$ & $0.711 \mathrm{E}-01$ \\
$08 / 23 / 83$ & & $0.1130 \mathrm{E}+01$ & $0.634 \mathrm{E}-01$ \\
$08 / 17 / 82$ & $0.4090 \mathrm{E}+00$ & $0.583 \mathrm{E}-01$ \\
$08 / 13 / 81$ & $0.6320 \mathrm{E}+00$ & $0.390 \mathrm{E}-01$ \\
$08 / 04 / 80$ & & $0.6530 \mathrm{E}+00$ & $0.840 \mathrm{E}-01$ \\
$09 / 12 / 79$ & & $0.7350 \mathrm{E}+00$ & $0.720 \mathrm{E}-01$ \\
$08 / 0 \mathrm{D} / 78$ & $0.5600 \mathrm{E}+00$ & $0.690 \mathrm{E}-01$ \\
$06 / 14 / 77$ & & $0.1430 \mathrm{E}+00$ & $0.400 \mathrm{E}-01$
\end{tabular}

Yakima

0512

Depth 0-1 in.

Cs-137

Sample

Dup. Result

$+/-2$

Date

No. $\mathrm{pCi} / \mathrm{q}$

std. Dev.

$05 / 21 / 87$

-

- - - - - - -

$0.1740 \mathrm{E}+00$

-----

$05 / 21 / 87$

$0.14 \mathrm{BOE}+00$

$0.2675-01$

$06 / 03 / 86$

$0.1140 \mathrm{E}+00$

$0.249 \mathrm{E}-01$ 
TABLE B. 3. (contd)

$\begin{array}{llll}\begin{array}{l}\text { Moges Lake } \\ \text { O500 }\end{array} & \text { Depth } & 0-1 \text { in. } & \text { Cg-137 } \\ \text { Sample } & \text { Dup. } & \text { Result } & +/-2 \\ \text { Date } & \text { No. } & \text { pCi/g } & \text { Std. Dev. } \\ -05 / 08 / 87 & 1 & 0.1300 E+00 & 0.282 E-01 \\ 05 / 08 / 87 & & 0.1790 E+00 & 0.276 E-01 \\ 05 / 23 / 86 & & 0.2410 E+00 & 0.285 E-01\end{array}$

othelio

0505

Depth 0-1 in.

Sample

Date

$-05 / 26 / B 2-1$

$05 / 25 / 87$

$05 / 02 / 86$

\section{Connell}

0503

Sample
Date

Depth 0-1 in.

Dup. Result

No.

$\mathrm{pCi} / \mathrm{g}$

$05 / 26 / 87$

$05 / 26 / 87$

$05 / 02 / 86$

1

--D-C-

$0.1780 \mathrm{E}+00$

$0.5220 \mathrm{E}+00$

$0.1740 \mathrm{E}+01$

\section{Washtucna}

$$
0501
$$

Depth 0-1 in.

Sample

Date

Dup.

Result

No.

$\mathrm{pCi} / \mathrm{g}$

$05 / 08 / 87$

$05 / 08 / 87$

$05 / 23 / 86$

1

$0.4230 \mathrm{E}+00$

$0.3280 \mathrm{E}+00$

$0.1200 \mathrm{E}+01$

Falla walla

0502

Depth 0-1 in.

Sample Dup.

No.

Result

$\mathrm{pCi} / \mathrm{g}$

$05 / 29 / 871$

$05 / 29 / 87$

$05 / 02 / 86$

$11 / 15 / 85$

$0.6770 \mathrm{0}-0$

$0.6770 \mathrm{E}-01$
$0.7130 \mathrm{E}-01$

$0.2480 E+00$

$0.2870 E+00$
Cs-137

$+/-2$

std. Dev.

- - - -

$0.292 E-01$

$0.399 E-01$

$0.559 \mathrm{E}-01$

$+/-2$

Std. Dev.

$0.717 \mathrm{E}-01$

60E-01

C6-137

$+/-2$

std. Dev.

$0.360 E-01$

$0.349 E-01$

$0.548 E-01$
Cs-137

$+/-2$

Std. Dev.

----D-

0.197E-01

$0.213 E-01$

$0.289 E-01$

$0.240 \mathrm{E}-01$
McNary Dam

0504 Depth o-1 in. Cs-137

Sample Dup. Result $+/-2$

Date No, pCl/g std. Dev.

$05 / 29 / 87 \quad 1 \quad 0.1650 \mathrm{E}+00 \quad 0.306 \mathrm{E}-01$

$05 / 29 / 87 \quad 0.4270 E+00 \quad 0.397 E-01$

$05 / 02 / 86 \quad 0.2810 \mathrm{E}+00 \quad 0.279 \mathrm{E}-01$

$11 / 15 / 85 \quad 0.5150 E+00 \quad 0.396 E-01$ 
TABLE B.4. Plutonlum-238 Concentrations in Soil at offsite Locations

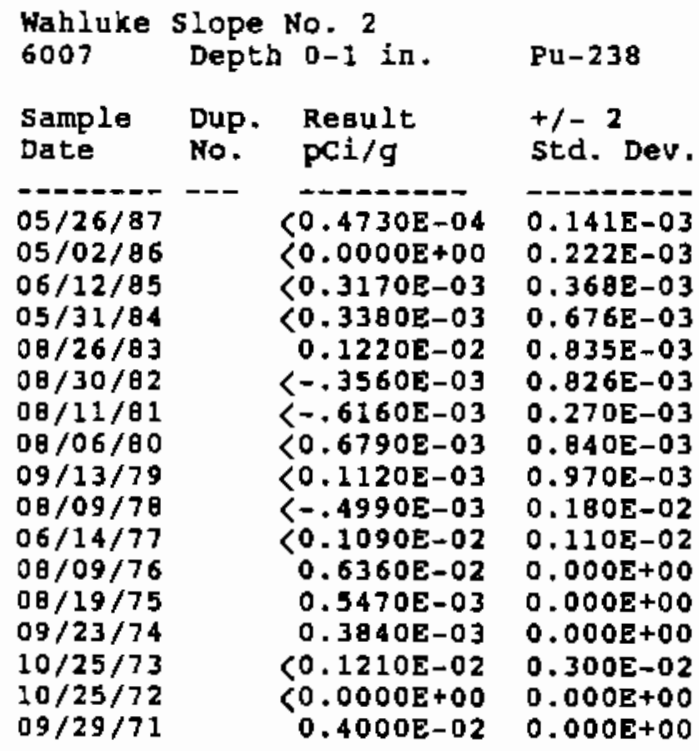

\begin{tabular}{llll} 
Wahluke slope No. 2 & \\
6030 & Depth $1-2$ ia. & Pu-238 \\
Sample & Dup. Result & $+/-2$ \\
Date & No. PCi/g & std. Dev. \\
\hline $10 / 25 / 72$ & & $0.6270 \mathrm{E}-02$ & $0.000 \mathrm{E}+00$ \\
$09 / 29 / 71$ & & $0.3000 \mathrm{E}-02$ & $0.000 \mathrm{E}+00$
\end{tabular}

Berg Ranch

\begin{tabular}{llll} 
6008 & Depth $0-1$ in. & Pu-23日 \\
Sample & Dup. & Result & $+/-2$ \\
Date & No. & pCi/g & std. Dev. \\
\hline $05 / 26 / 87$ & & $<0.1420 E-03$ & $0.174 E-03$ \\
$05 / 02 / 86$ & & $0.5740 E-03$ & $0.406 E-03$ \\
$06 / 26 / 85$ & $<0.1040 E-03$ & $0.104 E-03$ \\
$05 / 30 / 84$ & $0.3910 E-03$ & $0.334 E-03$ \\
$08 / 26 / 83$ & $0.1160 E-02$ & $0.848 E-03$ \\
$08 / 20 / 82$ & $<-.4990 E-03$ & $0.504 E-03$ \\
$08 / 11 / 81$ & 1 & $<0.4310 E-03$ & $0.610 E-03$ \\
$08 / 11 / 81$ & $<-1890 E-03$ & $0.340 E-03$ \\
$08 / 06 / 80$ & $0.1420 E-02$ & $0.890 E-03$ \\
$09 / 13 / 79$ & $<0.1490 E-03$ & $0.760 E-03$ \\
$09 / 09 / 78$ & $<-.4040 E-03$ & $0.160 E-02$ \\
$06 / 14 / 77$ & $0.3940 E-02$ & $0.320 E-02$ \\
$08 / 09 / 76$ & $0.4440 E-02$ & $0.000 E+00$ \\
$08 / 19 / 75$ & $<-.2800 E-03$ & $0.000 E+00$ \\
$09 / 23 / 74$ & $0.3120 E-03$ & $0.000 E+00$ \\
$10 / 25 / 73$ & $<0.1330 E-02$ & $0.300 E-02$ \\
$10 / 25 / 72$ & $0.6010 E-03$ & $0.000 E+00$ \\
$09 / 29 / 71$ & $0.6700 E-02$ & $0.000 E+00$
\end{tabular}

\begin{tabular}{llll}
$\begin{array}{l}\text { Berg Ranch } \\
6031\end{array}$ & Depth & $1-2$ in. & Pu-238 \\
& & & \\
Sample & Dup. & Result & $+/-2$ \\
Date & No. & pci/g & Std. Dev. \\
\hline $10 / 25 / 72$ & & $0.1600 E-01$ & $0.000 E+00$ \\
$09 / 29 / 71$ & & $0.3000 E-02$ & $0.000 E+00$
\end{tabular}

Cook 日roa. Dairy Farm

\begin{tabular}{llll}
6219 & Deptb $0-1$ In. & Pu-238 \\
Sample & Dup. Result & $+/-2$ \\
Date & No. & pc1/g & Std. Dev. \\
\hdashline $09 / 25 / 74$ & & $<0.00008+00$ & $0.000 E+00$
\end{tabular}

Ringold

6009

Depth $0-1$ in.

Pu $=238$

Sample

Dup.

Reeult

$+1-2$

Date

No.

$\mathrm{pCi} / \mathrm{g}$

Std. Dev.

$05 / 15 / 87$

$05 / 22 / 86$

$07 / 08 / 85$

$05 / 30 / 84$

$08 / 18 / 83$

$0 \theta / 20 / 82$

$08 / 12 / 81$

$08 / 06 / 80$

$09 / 13 / 79$

$08 / 09 / 7 \theta$

$06 / 14 / 77$

$08 / 09 / 76$

$08 / 19 / 75$

$10 / 25 / 73$

$10 / 25 / 72$

$10 / 01 / 71$

$0.7300 \mathrm{E}-03$

$<0.8910 E-04$

<0.2670E-03

$0.5320 E-03$

$<0.0000 \mathrm{E}+00$

$<-.3730 E-03$

$<0.5250 E-03$

$0.1520 \mathrm{E}-02$

$0.2220 \mathrm{E}-02$

$<0.1070 \mathrm{E}-02$

$0.3220 \mathrm{E}-02$

$0.0970 \mathrm{E}-03$

0.1220E-02

$<0.1340 \mathrm{E}-03$

$0.5360 \mathrm{E}-02$

$0,1900 \mathrm{E}-02$

$0.359 \mathrm{E}-03$

$0.103 E-03$

$0.275 E-03$

$0.362 E-03$

$0.0008+00$

$0.6328-03$

$0.640 E-03$

$0.950 \mathrm{E}-03$

$0.1208-02$

$0.150 \mathrm{E}-02$

$0.260 \mathrm{E}-02$

$0.000 \mathrm{E}+00$

$0.000 \mathrm{E}+00$

$0.3008-02$

$0.000 \mathrm{E}+00$

$0.0008+00$

Ringold

\section{2}

Sample

Date

$10 / 25 / 72$

$10 / 01 / 71$.
Depth 1-2 in.

Dup. Result

No.

$\mathrm{pCi} / \mathrm{g}$

$0.1590 \mathrm{E}-01$

$0.2900 E-02$
Pu-23B

$+/-2$

std. Dev.

$0.0008+00$

$0.000 \mathrm{E}+00$ 


\section{TABLE B.4. (contd)}

\begin{tabular}{|c|c|c|c|}
\hline $\begin{array}{l}\text { End of } \\
6360\end{array}$ & $\begin{array}{l}\text { Fir } \\
\text { Dept }\end{array}$ & o-1 in. & $P u-238$ \\
\hline $\begin{array}{l}\text { Sample } \\
\text { jate }\end{array}$ & $\begin{array}{l}\text { Dup. } \\
\text { No. }\end{array}$ & $\begin{array}{l}\text { Result } \\
\text { pCi/g }\end{array}$ & $\begin{array}{l}+/-2 \\
\text { std. Dev. }\end{array}$ \\
\hline $\begin{array}{l}-05 / 15 / 87 \\
05 / 22 / 86 \\
07 / 03 / 85 \\
05 / 31 / 84 \\
08 / 18 / 83 \\
08 / 20 / 82 \\
00 / 12 / 81 \\
08 / 06 / 80 \\
09 / 13 / 79 \\
08 / 09 / 78 \\
06 / 14 / 77\end{array}$ & --- & $\begin{array}{r}<0.5700 \mathrm{E}-04 \\
0.4120 \mathrm{E}-03 \\
0.2000 \mathrm{E}-03 \\
\langle 0.4970 \mathrm{E}-03 \\
\langle 0.3420 \mathrm{E}-03 \\
\langle-.8440 \mathrm{E}-04 \\
0.9090 \mathrm{E}-03 \\
\langle 0.1220 \mathrm{E}-03 \\
<-.1930 \mathrm{E}-03 \\
<0.9380 \mathrm{E}-03 \\
<-.3080 \mathrm{E}-03\end{array}$ & $\begin{array}{l}-.12 \mathrm{E}-03 \\
0.126 \mathrm{E}-03 \\
0.220 \mathrm{E}-03 \\
0.179 \mathrm{E}-03 \\
0.702 \mathrm{E}-03 \\
0.395 \mathrm{E}-03 \\
0.562 \mathrm{E}-03 \\
0.660 \mathrm{E}-03 \\
0.650 \mathrm{E}-03 \\
0.770 \mathrm{E}-03 \\
0.170 \mathrm{E}-02 \\
0.720 \mathrm{E}-03\end{array}$ \\
\hline
\end{tabular}

Baxter substation

$\begin{array}{lll}6220 & \text { Depth } 0-1 \mathrm{in} . \\ \text { Sample } & \text { Dup. Result } \\ \text { Date } & \text { No. } & \text { pCi/g } \\ -06 / 14 / 77 & & <0.2510 \mathrm{E}-03 \\ 09 / 25 / 74 & & 0.1060 \mathrm{E}-01\end{array}$

Pu-238

$+1-2$

std. Dev.

$0.760 \mathrm{E}-03$

$0.000 E+00$

\section{Pettett Dairy Farm}

$\begin{array}{llll}6359 & \text { Depth } 0-1 \text { in. } & \text { Pu-238 } \\ & & & \\ \text { Sample } & \text { Dup. } & \text { Result } & +/-2 \\ \text { Date } & \text { No. } & \text { pCi/g } & \text { std. Dev. } \\ -06 / 14 / 77 & & 0.2690 \mathrm{E}-02 & 0.140 \mathrm{E}-02\end{array}$

Taylor Flatg No. 1

$\begin{array}{llll}6420 & \text { Depth } 0-1 \text { in. } & \text { Pu-238 } \\ \text { Sample } & \text { Dup. } & \text { Regult } & +/-2 \\ \text { Date } & \text { No. } & \text { pCi } / g & \text { std. Dev. } \\ -08 / 18 / 82 & & <-.4290 E-04 & 0.588 E-03 \\ 08 / 14 / 81 & & 0.1980 E-02 & 0.690 E-03 \\ 01 / 22 / 81 & & 0.7160 E-03 & 0.550 E-03 \\ 08 / 06 / 80 & & 0.7280 E-02 & 0.150 E-02 \\ 09 / 13 / 79 & <-.3690 E-03 & 0.940 E-03 \\ 08 / 10 / 78 & & 0.3170 E-02 & 0.120 E-02\end{array}$

\begin{tabular}{|c|c|c|c|}
\hline 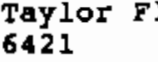 & Dept & $0-1$ in. & $\mathrm{Pu}-238$ \\
\hline $\begin{array}{l}\text { ample } \\
\text { ate }\end{array}$ & $\begin{array}{l}\text { Dup. } \\
\text { No. }\end{array}$ & $\begin{array}{l}\text { Result } \\
\text { pCi/g }\end{array}$ & $\begin{array}{l}+/-2 \\
\text { std. Dev. }\end{array}$ \\
\hline $\begin{array}{l}0-10- \\
05 / 15 / 87 \\
05 / 22 / 86 \\
07 / 08 / 85 \\
05 / 30 / 84 \\
08 / 26 / 83 \\
08 / 20 / 82 \\
11 / 03 / 81 \\
11 / 03 / 81 \\
11 / 03 / 81 \\
08 / 14 / 81 \\
01 / 22 / 81 \\
08 / 06 / 80 \\
09 / 13 / 79 \\
08 / 10 / 78\end{array}$ & $-\infty-$ & $\begin{array}{r}-0.4620 \mathrm{E}-03 \\
<0.5290 \mathrm{E}-03 \\
0.2240 \mathrm{E}-03 \\
<0.8470 \mathrm{E}-04 \\
0.1120 \mathrm{E}-02 \\
<0.9650 \mathrm{E}-03 \\
<-.4710 \mathrm{E}-03 \\
<-.5710 \mathrm{E}-03 \\
<-.1520 \mathrm{E}-04 \\
0.1650 \mathrm{E}-02 \\
0.2190 \mathrm{E}-02 \\
0.4670 \mathrm{E}-02 \\
0.9910 \mathrm{E}-03 \\
<0.1040 \mathrm{E}-03\end{array}$ & $\begin{array}{l}-.257 \mathrm{E}-03 \\
0.529 \mathrm{E}-03 \\
0.177 \mathrm{E}-03 \\
0.140 \mathrm{E}-03 \\
0.100 \mathrm{E}-02 \\
0.124 \mathrm{E}-02 \\
0.260 \mathrm{E}-03 \\
0.260 \mathrm{E}-03 \\
0.350 \mathrm{E}-03 \\
0.620 \mathrm{E}-03 \\
0.800 \mathrm{E}-03 \\
0.170 \mathrm{E}-02 \\
0.740 \mathrm{E}-03 \\
0.300 \mathrm{E}-02\end{array}$ \\
\hline
\end{tabular}

\begin{tabular}{|c|c|c|c|}
\hline $\begin{array}{l}\text { Sagemore } \\
6358\end{array}$ & $\begin{array}{l}\text { FarmB } \\
\text { Depth }\end{array}$ & 0-1 in. & $\mathrm{Pu}-238$ \\
\hline $\begin{array}{l}\text { Sample } \\
\text { Date }\end{array}$ & $\begin{array}{l}\text { Dup. } \\
\text { No. }\end{array}$ & $\begin{array}{l}\text { Reau 1t } \\
\mathrm{pCi} / \mathrm{g}\end{array}$ & $\begin{array}{l}+/-2 \\
\text { Std. Dev. }\end{array}$ \\
\hline $\begin{array}{l}-\overline{0} / 15 / 87 \\
05 / 22 / 86 \\
07 / 08 / 85 \\
05 / 30 / 84 \\
08 / 25 / 83 \\
08 / 18 / 82 \\
11 / 02 / 81 \\
08 / 12 / 81 \\
08 / 06 / 80 \\
09 / 13 / 79 \\
08 / 09 / 78 \\
06 / 14 / 77\end{array}$ & --- & $\begin{array}{r}<0.2760 \mathrm{E}-05 \\
0.4280 \mathrm{E}-03 \\
<0.8870 \mathrm{E}-04 \\
0.6040 \mathrm{E}-03 \\
0.1130 \mathrm{E}-02 \\
\langle-.7300 \mathrm{E}-03 \\
\langle-.4360 \mathrm{E}-03 \\
0.1920 \mathrm{E}-02 \\
0.6880 \mathrm{E}-02 \\
\angle 0.5940 \mathrm{E}-03 \\
<-.6800 \mathrm{E}-03 \\
<0.9530 \mathrm{E}-03\end{array}$ & $\begin{array}{l}0.779 \mathrm{E}-04 \\
0.214 \mathrm{E}-03 \\
0.159 \mathrm{E}-03 \\
0.382 \mathrm{E}-03 \\
0.563 \mathrm{E}-03 \\
0.296 \mathrm{E}-03 \\
0.350 \mathrm{E}-03 \\
0.670 \mathrm{E}-03 \\
0.150 \mathrm{E}-02 \\
0.140 \mathrm{E}-02 \\
0.130 \mathrm{E}-02 \\
0.130 \mathrm{E}-02\end{array}$ \\
\hline
\end{tabular}


TABLE B.4. (contd)

\begin{tabular}{llll}
$\begin{array}{l}\text { Sagemore } \\
\text { 0819 }\end{array}$ & $\begin{array}{l}\text { Farms } \\
\text { Depth }\end{array}$ (QC Dupl. B) \\
& & & Pu-238 \\
Sample & Dup. & Result & $+/-2$ \\
Date & No. & pCi/g & Std. Dev. \\
\hline $05 / 15 / 87$ & & $-0.6240 E-04$ & $0.135 E-03$ \\
$05 / 22 / 86$ & & $0.6350 E-03$ & $0.276 E-03$ \\
$07 / 08 / 85$ & & $0.2620 E-03$ & $0.220 E-03$ \\
$05 / 30 / 84$ & & $0.0190 E-03$ & $0.579 E-03$ \\
$00 / 25 / 83$ & $0.1320 E-02$ & $0.116 E-02$ \\
$08 / 18 / 82$ & & $0.1140 E-02$ & $0.890 E-03$
\end{tabular}

Byers Landing Pump House 6010 Depth $0-1$ in.

Pu-238

\begin{tabular}{llll} 
Sample & Dup. & $\begin{array}{l}\text { Regult } \\
\text { pci }\end{array}$ & $+/-2$ \\
Date & No. & Std. Dev. \\
\hline $09 / 25 / 74$ & & $0.1320 \mathrm{E}-02$ & $0.000 \mathrm{E}+00$ \\
$10 / 25 / 73$ & & $<0.1520 \mathrm{E}-02$ & $0.300 \mathrm{E}-02$ \\
$10 / 25 / 72$ & & $<0.0000 \mathrm{E}+00$ & $0.000 \mathrm{E}+00$ \\
$10 / 01 / 71$ & & $0.2800 \mathrm{E}-02$ & $0.000 \mathrm{E}+00$
\end{tabular}

Byers Landing Pump Houge 6033 Depth $1-2$ in.

Pu-238

\begin{tabular}{llll} 
Sample & Dup. & Regult & $+/-{ }^{2}$ \\
Date & No. & pCi/g & Std. Dev. \\
\hline $10 / 25 / 72$ & & $<0.0000 \mathrm{E}+00$ & $0.000 \mathrm{D}+00$ \\
$10 / 01 / 71$ & & $0.1100 \mathrm{D}-02$ & $0.000 \mathrm{E}+00$
\end{tabular}

Byers Landing

\begin{tabular}{llll}
6011 & Depth $0-1$ in. & Pu-238 \\
Sample & Dup. & Regult & $+/-2$ \\
Date & No. & pCi/g & std. Dev. \\
\hline $05 / 15 / 87$ & & $<0.6590 E-04$ & $0.106 \mathrm{E}-03$ \\
$05 / 22 / 86$ & & $0.3810 \mathrm{E}-03$ & $0.211 \mathrm{E}-03$ \\
$06 / 19 / 85$ & $0.1190 \mathrm{E}-02$ & $0.451 \mathrm{E}-03$ \\
$05 / 30 / 84$ & $0.3600 \mathrm{E}-02$ & $0.294 \mathrm{E}-02$ \\
$00 / 25 / 83$ & $<0.3620 \mathrm{E}-03$ & $0.362 \mathrm{E}-03$ \\
$08 / 18 / 82$ & $<0.6040 \mathrm{E}-03$ & $0.871 \mathrm{E}-03$ \\
$08 / 12 / 81$ & $<-.5060 \mathrm{E}-03$ & $0.380 \mathrm{E}-03$ \\
$08 / 06 / 80$ & & & \\
$09 / 13 / 79$ & $<0.3290 \mathrm{E}-03$ & $0.720 \mathrm{E}-03$ \\
$00 / 09 / 78$ & $<0.3460 \mathrm{E}-02$ & $0.500 \mathrm{E}-02$ \\
$06 / 14 / 77$ & $<0.2760 \mathrm{E}-03$ & $0.890 \mathrm{E}-03$ \\
$08 / 09 / 76$ & $0.1360 \mathrm{E}-02$ & $0.000 \mathrm{E}+00$ \\
$09 / 25 / 74$ & $0.2650 \mathrm{E}-03$ & $0.000 \mathrm{E}+00$ \\
$10 / 25 / 73$ & $<0.6750 \mathrm{E}-03$ & $0.300 \mathrm{E}-02$ \\
$10 / 25 / 72$ & $0.9110 \mathrm{E}-03$ & $0.000 \mathrm{E}+00$ \\
$10 / 01 / 71$ & $0.1600 \mathrm{E}-02$ & $0.000 \mathrm{E}+00$
\end{tabular}

\begin{tabular}{llll}
$\begin{array}{l}\text { Byerg Landing } \\
6034\end{array}$ & & \\
& Depth & $1-2$ in. & Pu-238 \\
Sample Dup. Regult & $+/-2$ \\
Date & No. & pCilg & Std. Dev. \\
\hdashline $10 / 25 / 72$ & & $0.2880 \mathrm{E}-02$ & $0.000 \mathrm{E}+00$ \\
$10 / 01 / 71$ & & $0.2440 \mathrm{E}-02$ & $0.000 \mathrm{E}+00$
\end{tabular}

Riverview (Harrio Earm)

6361 Depth $0-1$ in.

$\mathrm{Pu}-238$

\begin{tabular}{|c|c|c|c|}
\hline $\begin{array}{l}\text { Sample } \\
\text { Date }\end{array}$ & $\begin{array}{l}\text { Dup. } \\
\text { No. }\end{array}$ & $\begin{array}{l}\text { Result } \\
\mathrm{pCi} / \mathrm{g}\end{array}$ & $\begin{array}{l}+/-2 \\
\text { std. Dev. }\end{array}$ \\
\hline $\begin{array}{l}05 / 15 / 87 \\
05 / 22 / 86 \\
06 / 19 / 85 \\
05 / 30 / \theta 4 \\
08 / 18 / \theta 3 \\
08 / 18 / 82 \\
0 \theta / 12 / 81 \\
0 \theta / 06 / 80 \\
09 / 13 / 79 \\
08 / 09 / 78 \\
06 / 14 / 77\end{array}$ & --- & $\begin{array}{r}0.3240 \mathrm{E}-03 \\
0.2670 \mathrm{E}-03 \\
<0.2290 \mathrm{E}-03 \\
0.2260 \mathrm{E}-02 \\
<0.0230 \mathrm{E}-03 \\
\langle-.2710 \mathrm{E}-03 \\
\langle-.3580 \mathrm{E}-03 \\
\langle 0.5170 \mathrm{E}-03 \\
<0.5890 \mathrm{E}-04 \\
<0.9790 \mathrm{E}-03 \\
0.3170 \mathrm{O}-02\end{array}$ & $\begin{array}{l}-0.194 \mathrm{E}-03 \\
0.197 \mathrm{E}-03 \\
0.242 \mathrm{E}-03 \\
0.202 \mathrm{E}-02 \\
0.106 \mathrm{E}-02 \\
0.633 \mathrm{E}-03 \\
0.300 \mathrm{E}-03 \\
0.560 \mathrm{E}-03 \\
0.750 \mathrm{E}-03 \\
0.130 \mathrm{E}-02 \\
0.280 \mathrm{E}-02\end{array}$ \\
\hline
\end{tabular}

Rivervlew Control Plot 55

6012 Depth $0-1$ in.

Pu-238

Sample Dup. Result $t /-2$

Date No. pCi/g std. Dev.

-

$09 / 25 / 74<0.0000 \mathrm{E}+00 \quad 0.000 \mathrm{E}+00$

$10 / 25 / 73 \quad 0.6270 E-02 \quad 0.3008-02$

$10 / 25 / 72 \quad 0.2200 E-02 \quad 0.000 E+00$

$10 / 01 / 71 \quad 0.5000 E-02 \quad 0.000 E+00$

\begin{tabular}{|c|c|c|c|}
\hline $\begin{array}{l}\text { Rivervier } \\
6035\end{array}$ & $\begin{array}{l}\text { Cont } \\
\text { Depth }\end{array}$ & $\begin{array}{l}\text { rol plot } 55 \\
1-2 \text { in. }\end{array}$ & $\mathrm{Pu}-238$ \\
\hline $\begin{array}{l}\text { Sample } \\
\text { Date }\end{array}$ & $\begin{array}{l}\text { Dup. } \\
\text { No. }\end{array}$ & $\begin{array}{l}\text { Result } \\
\text { pci/g }\end{array}$ & $\begin{array}{l}+/-2 \\
\text { std. Dev. }\end{array}$ \\
\hline $\begin{array}{l}-10 / 25 / 72 \\
10 / 01 / 71\end{array}$ & -- & $<0.0000 \mathrm{E}+00$ & $\begin{array}{l}0.000 E+00 \\
0.000 E+00\end{array}$ \\
\hline
\end{tabular}


TABLE B.4. (contd)

N Richland Control Plot 56

$\begin{array}{llll}6013 & \text { Depth } 0-1 \text { in. } & \text { Pu-238 } \\ \text { Sample } & \text { Dup. } & \text { Result } & +/-2 \\ \text { Date } & \text { No. } & \text { pCi } / g & \text { std. Dev. } \\ -09 / 25 / 74 & & 0.4510 \mathrm{E}-03 & 0.000 \mathrm{E}+00 \\ 10 / 25 / 73 & & <0.7480 \mathrm{E}-03 & 0.300 \mathrm{E}-02 \\ 10 / 25 / 72 & & 0.1160 \mathrm{E}-01 & 0.000 \mathrm{E}+00 \\ 09 / 30 / 71 & & 0.3700 \mathrm{E}-02 & 0.000 \mathrm{E}+00\end{array}$

N Richland Control Plot 56

$\begin{array}{llll}6036 & \text { Depth } 1-2 \text { in. } & \text { Pu-23日 } \\ \text { Sample } & \text { Dup. } & \text { Result } & +/-2 \\ \text { Date } & \text { No. } & \text { pCi/g } & \text { Std. Dev. } \\ -10 / 25 / 72 & & 0.9740 \mathrm{E}-02 & 0.000 \mathrm{E}+00 \\ 09 / 30 / 71 & & 0.2680 \mathrm{E}-02 & 0.000 \mathrm{E}+00\end{array}$

NE Corner of Exxon Site

6200 Depth $0-1$ in.

$\mathrm{Pu}-238$

\begin{tabular}{llll} 
Sample & Dup. & Regult & $+/-2$ \\
Date & No. & pCi/g & std. Dev. \\
\hdashline $08 / 09 / 76$ & & $0.6610 \mathrm{E}-03$ & $0.000 \mathrm{E}+00$ \\
$08 / 19 / 75$ & & $0.2870 \mathrm{E}-03$ & $0.000 \mathrm{E}+00$
\end{tabular}

Benton city

\begin{tabular}{|c|c|c|c|}
\hline 000 & Depth & h $0-1$ in. & $P u-23 \theta$ \\
\hline $\begin{array}{l}\text { ample } \\
\text { ate }\end{array}$ & $\begin{array}{l}\text { Dup. } \\
\text { No. }\end{array}$ & $\begin{array}{l}\text { Result } \\
\mathrm{p} \text { Ei/g }\end{array}$ & $\begin{array}{l}+/-2 \\
\text { std. Dev. }\end{array}$ \\
\hline $\begin{array}{l}------- \\
05 / 20 / 87 \\
05 / 23 / 86 \\
07 / 03 / 85 \\
06 / 06 / 84 \\
08 / 23 / 83 \\
08 / 16 / 82 \\
08 / 13 / 81 \\
08 / 04 / 80 \\
09 / 12 / 79 \\
08 / 08 / 78 \\
06 / 14 / 77 \\
09 / 25 / 74 \\
10 / 25 / 73\end{array}$ & --- & $\begin{array}{r}- \\
0.2780 E-03 \\
0.6200 E-03 \\
0.5620 E-03 \\
<0.3500 E-03 \\
0.6640 E-03 \\
<0.4640 E-03 \\
<-.4310 E-03 \\
0.1940 E-02 \\
<0.2940 E-03 \\
<0.0510 E-04 \\
<-.1930 E-04 \\
0.1770 E-02 \\
<0.0000 E+00\end{array}$ & $\begin{array}{l}=-.--- \\
0.206 \mathrm{E}-03 \\
0.312 \mathrm{E}-03 \\
0.371 \mathrm{E}-03 \\
0.368 \mathrm{E}-03 \\
0.379 \mathrm{E}-03 \\
0.820 \mathrm{E}-03 \\
0.410 \mathrm{E}-03 \\
0.110 \mathrm{E}-02 \\
0.390 \mathrm{E}-03 \\
0.770 \mathrm{E}-03 \\
0.730 \mathrm{E}-03 \\
0.000 \mathrm{E}+00 \\
0.300 \mathrm{E}-02\end{array}$ \\
\hline $\begin{array}{l}10 / 25 / 72 \\
09 / 30 / 71\end{array}$ & & $\begin{array}{l}0.1670 \mathrm{E}-02 \\
0.1890 \mathrm{E}-02\end{array}$ & $\begin{array}{l}0.000 \mathrm{E}+00 \\
0.000 \mathrm{E}+00\end{array}$ \\
\hline
\end{tabular}

$\begin{array}{llll}\begin{array}{l}\text { Benton City } \\ 6023\end{array} & \text { Depth } 1-2 \text { in. } & \text { Pu-238 } \\ & & & \\ \text { Sample } & \text { Dup. Regult } & +/-2 \\ \text { Date } & \text { No. } & \text { pCi/g } & \text { std. Dev. } \\ -10 / 25 / 72 & & <0.0000 E+00 & 0.000 E+00 \\ 09 / 30 / 71 & & 0.1200 E-02 & 0.000 E+00\end{array}$

Benton City (QC Dupl. A) 0820 Depth 0-1 in.

$\mathrm{Pu}-23 \theta$

\begin{tabular}{|c|c|c|c|}
\hline $\begin{array}{l}\text { Sample } \\
\text { Date }\end{array}$ & $\begin{array}{l}\text { Dup. } \\
\text { No. }\end{array}$ & $\begin{array}{l}\text { Result } \\
\text { pCi/g }\end{array}$ & $\begin{array}{l}+/-2 \\
\text { std. Dev. }\end{array}$ \\
\hline$/ 17 / 82$ & --- & $0.7800 \mathrm{E}-03$ & \\
\hline
\end{tabular}

Benton City (QC Dupl. B) 0821 Depth $0-1$ in.

Pu-23日

Sample Dup. Result $+/-2$ Date No. pCi/g std. Dev.

-------- -. - -

$08 / 17 / 82 \quad<0.1730 \mathrm{E}-03 \quad 0.652 \mathrm{E}-03$

Sunnyside

6357 Depth $0-1$ in. Pu-238

Sample Dup. Result $+/-2$

Date No. pCi/g std. Dev.

$\begin{array}{llll}05 / 21 / 87 & 1 & 0.2380 \mathrm{E}-03 & 0.220 \mathrm{E}-03\end{array}$

$06 / 03 / 86<0.0000 \mathrm{E}+00 \quad 0.182 \mathrm{E}-03$

$06 / 17 / 85<0.1990 E-03 \quad 0.211 E-03$

$05 / 22 / 84 \quad 0.10708-02 \quad 0.536 \mathrm{E}-03$

$08 / 23 / 83<0.20008-03 \quad 0.401 E-03$

$08 / 17 / 82<0.5260 E-03 \quad 0.785 E-03$

$08 / 13 / 81<-.1160 \mathrm{~L}-03 \quad 0.300 \mathrm{E}-03$

$08 / 04 / 80 \quad 0.22108-02 \quad 0.120 E-02$

$09 / 12 / 79 \quad<0.9400 E-04 \quad 0.720 E-03$

$08 / 08 / 78<-.2210 \mathrm{E}-03 \quad 0.130 \mathrm{E}-02$

$06 / 14 / 77 \quad 0.12$ Q0E +02 0.110E-02

\begin{tabular}{llll}
$\begin{array}{l}\text { Yakima } \\
\text { 0512 }\end{array}$ & Depth $0-1$ in. & Pu-238 \\
Sample & Dup. & Regult & $+/-2$ \\
Date & No. & pCi/g & std. Dev. \\
\hline $05 / 21 / 87$ & 1 & $<0.9420 E-04$ & $0.147 \mathrm{E}-03$ \\
$05 / 21 / 87$ & & $<0.1420 \mathrm{E}-03$ & $0.194 \mathrm{E}-03$ \\
$06 / 03 / 86$ & & $0.4520 \mathrm{E}-03$ & $0.369 \mathrm{E}-03$
\end{tabular}


TABLE B.4. (contd)

\begin{tabular}{|c|c|c|c|}
\hline $\begin{array}{l}\text { Moses Lal } \\
0500\end{array}$ & ke & $0-1$ in. & $\mathrm{Pu}-238$ \\
\hline $\begin{array}{l}\text { Sample } \\
\text { Date }\end{array}$ & $\begin{array}{l}\text { Dup. } \\
\text { No. }\end{array}$ & $\begin{array}{l}\text { Result } \\
\mathrm{pCl} / \mathrm{g}\end{array}$ & $\begin{array}{l}+/-2 \\
\text { std. Dev. }\end{array}$ \\
\hline $\begin{array}{l}05 / 08 / 87 \\
05 / 08 / 87 \\
05 / 23 / 86\end{array}$ & --- & $\begin{array}{l}<0.9800 E-04 \\
<-.1810 \mathrm{E}-04 \\
0.3070 \mathrm{E}-03\end{array}$ & $\begin{array}{l}0.174 \mathrm{E}-03 \\
0.119 \mathrm{E}-03 \\
0.171 \mathrm{E}-03\end{array}$ \\
\hline $\begin{array}{l}\text { Othello } \\
0505\end{array}$ & Depth & $0-1$ in. & $P u-238$ \\
\hline $\begin{array}{l}\text { Sample } \\
\text { Date }\end{array}$ & $\begin{array}{l}\text { Dup. } \\
\text { No. }\end{array}$ & $\begin{array}{l}\text { Regult } \\
\text { pCi/g }\end{array}$ & $\begin{array}{l}+/-2 \\
\text { std. Dev. }\end{array}$ \\
\hline $\begin{array}{l}05 / 26 / 87 \\
05 / 26 / 87 \\
05 / 02 / 86\end{array}$ & --- & $\begin{array}{l}0.2940 \mathrm{E}-03 \\
0.4790 \mathrm{E}-03 \\
0.8410 \mathrm{E}-04\end{array}$ & $\begin{array}{l}0.245 E-03 \\
0.332 E-03 \\
0.971 E-04\end{array}$ \\
\hline $\begin{array}{l}\text { Connell } \\
0503\end{array}$ & Depth & $0-1$ in. & $\mathrm{Pu}-238$ \\
\hline $\begin{array}{l}\text { Sample } \\
\text { Date }\end{array}$ & $\begin{array}{l}\text { Dup. } \\
\text { No. }\end{array}$ & $\begin{array}{l}\text { Regult } \\
\mathrm{pCi} / \mathrm{g}\end{array}$ & $\begin{array}{l}+/{ }^{2} \\
\text { std. Dev. }\end{array}$ \\
\hline $\begin{array}{l}-------- \\
05 / 26 / 87 \\
05 / 26 / 87 \\
05 / 02 / 86\end{array}$ & $-\frac{-}{1}$ & $\begin{array}{l}0.2750 \mathrm{E}-03 \\
0.3370 \mathrm{E}-03 \\
0.1040 \mathrm{E}-02\end{array}$ & $\begin{array}{l}0.197 \mathrm{E}-03 \\
0.189 \mathrm{E}-03 \\
0.486 \mathrm{E}-03\end{array}$ \\
\hline
\end{tabular}

\begin{tabular}{llll}
$\begin{array}{l}\text { McNary Dam } \\
0504\end{array}$ & \multicolumn{1}{l}{ Depth $0-1$ in. } & Pu-238 \\
Sample & Dup. & Regult & $+/-2$ \\
Date & No. & pCi/g & std. Dev. \\
\hline $05 / 29 / 87$ & & $<0.1690 E-03$ & $0.181 E-03$ \\
$05 / 29 / 87$ & 1 & $<0.32208-04$ & $0.976 E-04$ \\
$05 / 02 / 86$ & & $0.2920 E-03$ & $0.215 E-03$ \\
$11 / 15 / 85$ & & $0.1430 E-02$ & $0.759 E-03$
\end{tabular}

\begin{tabular}{llll}
\multicolumn{2}{l}{ Waghtucna } \\
0501 & Depth $0-1$ in. & Pu-238 \\
Sample & Dup. & Result & $+/-2$ \\
Date & No. & pCi/g & std. Dev. \\
\hline $05 / 08 / 87$ & 1 & $<0.1460 \mathrm{E}-03$ & $0.199 \mathrm{E}-03$ \\
$05 / 00 / 87$ & & $0.1220 \mathrm{E}-03$ & $0.156 \mathrm{E}-03$ \\
$05 / 23 / 86$ & & $0.1080 \mathrm{E}-02$ & $0.364 \mathrm{E}-03$
\end{tabular}

Walla Walla

$\begin{array}{llll}0502 & \text { Depth } & 0-1 \text { in. } & \text { Pu-23日 } \\ \text { Sample } & \text { Dup. } & \text { Regult } & +/-2 \\ \text { Date } & \text { No. } & \text { pci/g } & \text { std. Dev. } \\ -05 / 29 / 87 & & --1600 E-04 & 0.726 E-04 \\ 05 / 29 / 87 & 1 & <0.1070 E-03 & 0.225 E-03 \\ 05 / 02 / 86 & & 0.3510 E-03 & 0.234 E-03 \\ 11 / 15 / 85 & & 0.8080 E-03 & 0.330 E-03\end{array}$


TABLE B.5. Plutonium-239,240 Concentrations in soil at offsite Locations

Wahluke slope No. 2 6007 Depth $0-1$ in.

$\mathrm{Pu}-239,240$

\begin{tabular}{lll} 
Sample & Dup. & Regult \\
Date & No. & pC1/g \\
\hline $05 / 26 / 87$ & & $0.2860 \mathrm{E}-02$ \\
$05 / 02 / 86$ & & $0.5520 \mathrm{E}-02$ \\
$06 / 12 / 85$ & $0.8720 \mathrm{E}-02$ \\
$05 / 31 / 84$ & $0.6080 \mathrm{E}-02$ \\
$08 / 26 / 83$ & $0.9560 \mathrm{E}-02$ \\
$08 / 30 / 82$ & $0.5030 \mathrm{E}-02$ \\
$08 / 11 / 81$ & $0.3670 \mathrm{E}-02$ \\
$08 / 06 / 80$ & $0.6320 \mathrm{E}-02$ \\
$09 / 13 / 79$ & $0.3960 \mathrm{E}-02$ \\
$08 / 09 / 78$ & $0.4580 \mathrm{E}-02$ \\
$06 / 14 / 77$ & $0.3350 \mathrm{E}-02$ \\
$08 / 09 / 76$ & $0.1000 \mathrm{E}-01$ \\
$08 / 19 / 75$ & $0.4140 \mathrm{E}-02$ \\
$09 / 23 / 74$ & $0.1340 \mathrm{E}-01$ \\
$10 / 25 / 73$ & $0.2310 \mathrm{E}-01$ \\
$10 / 25 / 72$ & $0.1000 \mathrm{E}-01$ \\
$09 / 29 / 71$ & $0.4000 \mathrm{E}-02$
\end{tabular}

Wabluke slope No, 2

6030 Depth $1-21 \mathrm{ln}$

\begin{tabular}{llll} 
Sample & Dup. & Regult & $+/-2$ \\
Date & No. & pCI/g & std. Dev. \\
\hline $10 / 25 / 72$ & & $0.3000 \mathrm{E}-02$ & $0.000 \mathrm{E}+00$ \\
$09 / 29 / 71$ & & $0.4000 \mathrm{E}-02$ & $0.000 \mathrm{E}+00$
\end{tabular}

Berg Ranch

\begin{tabular}{llll}
$600 \mathrm{~B}$ & Depth $0-1$ in. & Pu-239,240 \\
Sample & Dup. & Result & $+/-2$ \\
Date & No. & pC1/g & std. Dev. \\
\hline $05 / 26 / 87$ & & $0.6120 \mathrm{E}-02$ & $0.977 \mathrm{E}-03$ \\
$05 / 02 / 86$ & & $0.1200 \mathrm{E}-01$ & $0.151 \mathrm{E}-02$ \\
$06 / 26 / 83$ & & $0.1060 \mathrm{E}-01$ & $0.105 \mathrm{E}-02$ \\
$05 / 30 / 84$ & & $0.9700 \mathrm{E}-02$ & $0.150 \mathrm{E}-02$ \\
$08 / 26 / 83$ & & $0.1350 \mathrm{E}-01$ & $0.284 \mathrm{E}-02$ \\
$08 / 20 / 82$ & & $0.1180 \mathrm{E}-01$ & $0.233 \mathrm{E}-02$ \\
$08 / 11 / 81$ & 1 & $0.8680 \mathrm{E}-02$ & $0.150 \mathrm{E}-02$ \\
$08 / 11 / 81$ & & $0.9680 \mathrm{E}-02$ & $0.110 \mathrm{E}-02$ \\
$08 / 06 / 80$ & & $0.8870 \mathrm{E}-02$ & $0.100 \mathrm{E}-02$ \\
$09 / 13 / 79$ & & $0.1300 \mathrm{E}-01$ & $0.260 \mathrm{E}-02$ \\
$08 / 09 / 78$ & & $0.4260 \mathrm{E}-02$ & $0.270 \mathrm{E}-02$ \\
$06 / 14 / 77$ & & $0.2680 \mathrm{E}-01$ & $0.670 \mathrm{E}-02$ \\
$08 / 09 / 76$ & & $0.5610 \mathrm{E}-02$ & $0.000 \mathrm{E}+00$ \\
$08 / 19 / 75$ & & $0.8180 \mathrm{E}-02$ & $0.000 \mathrm{E}+00$ \\
$09 / 23 / 74$ & & $0.7150 \mathrm{E}-02$ & $0.000 \mathrm{E}+00$ \\
$10 / 25 / 73$ & & $0.1580 \mathrm{E}-01$ & $0.000 \mathrm{E}+00$ \\
$10 / 25 / 72$ & & $0.7000 \mathrm{E}-02$ & $0.000 \mathrm{E}+00$ \\
$09 / 30 / 71$ & & $0.1200 \mathrm{E}-01$ & $0.000 \mathrm{E}+00$
\end{tabular}

Berg Ranch

\begin{tabular}{llll}
6031 & Depth $1-2$ in. & Pu-239,240 \\
Sample & Dup. & Result & $+/-2$ \\
Date & No: & pc1/g & Std. Dev. \\
\hline $10 / 25 / 72$ & & $0.2000 \mathrm{E}-02$ & $0.000 \mathrm{E}+00$ \\
$09 / 29 / 71$ & & $0.3000 \mathrm{E}-02$ & $0.000 \mathrm{E}+00$
\end{tabular}

Cook Brob. Dalry Farm 6219 Depth 0-1 in.

Pu-239,240

Sample Dup. Result $\quad+/-2$

Date No. pCl/g std. Dev.

$09 / 25 / 74=0.3110 \mathrm{E}-02 \quad 0.000 \mathrm{E}+00$

RIngold

6009 Depth $0-1$ in. Pu-239,240

\begin{tabular}{|c|c|c|c|}
\hline $\begin{array}{l}\text { Sample } \\
\text { Date }\end{array}$ & $\begin{array}{l}\text { Dup. } \\
\text { No. }\end{array}$ & $\begin{array}{l}\text { Result } \\
\text { pCi/g }\end{array}$ & $\begin{array}{l}+/-2 \\
\text { std. Dev. }\end{array}$ \\
\hline $\begin{array}{l}-05 / 15 / 87 \\
05 / 22 / 86 \\
05 / 22 \\
07 / 08 / 85 \\
05 / 30 / 84 \\
08 / 18 / 83 \\
08 / 20 / 82 \\
08 / 12 / 81 \\
08 / 06 / 80 \\
09 / 13 / 79 \\
08 / 09 / 78 \\
06 / 14 / 77 \\
08 / 09 / 76 \\
08 / 19 / 75\end{array}$ & -- & $\begin{array}{l}-.-168 \mathrm{E}-01 \\
0.1680 \\
0.6190 \mathrm{E}-02 \\
0.1700 \mathrm{E}-01 \\
0.7450 \mathrm{E}-02 \\
0.2770 \mathrm{E}-01 \\
0.1290 \mathrm{E}-01 \\
0.2140 \mathrm{E}-01 \\
0.1790 \mathrm{E}-01 \\
0.1630 \mathrm{E}-01 \\
0.1740 \mathrm{E}-01 \\
0.4640 \mathrm{E}-02 \\
0.3440 \mathrm{E}-02 \\
0.4430 \mathrm{E}-02\end{array}$ & $\begin{array}{l}0.166 \mathrm{E}-02 \\
0.857 \mathrm{E}-03 \\
0.162 \mathrm{E}-02 \\
0.123 \mathrm{E}-02 \\
0.518 \mathrm{E}-02 \\
0.243 \mathrm{E}-02 \\
0.220 \mathrm{E}-02 \\
0.260 \mathrm{E}-02 \\
0.290 \mathrm{E}-02 \\
0.370 \mathrm{E}-02 \\
0.280 \mathrm{E}-02 \\
0.000 \mathrm{E}+00 \\
0.000 \mathrm{E}+00\end{array}$ \\
\hline $\begin{array}{l}10 / 25 / 73 \\
10 / 25 / 72 \\
10 / 01 / 71\end{array}$ & & $\begin{array}{r}<0.2900 E-04 \\
0.8000 E-02 \\
0.6000 E-02\end{array}$ & $\begin{array}{l}0.000 E+00 \\
0.000 E+00 \\
0.000 E+00\end{array}$ \\
\hline
\end{tabular}

Ringold

6032 Depth 1-2 in. Pu-239,240

Sample Dup. Regult $+/-2$

Date No. pCi/g std. Dev.

$\begin{array}{llll}10 / 25 / 72 & 0.2200 \mathrm{E}-01 & 0.000 \mathrm{E}+00\end{array}$

$10 / 01 / 71 \quad 0.8000 \mathrm{E}-02 \quad 0.000 \mathrm{E}+00$ 
TABLE B.5. (contd)

\begin{tabular}{llll}
$\begin{array}{l}\text { W End of Fir Road } \\
6360\end{array}$ & Repth $0-1$ in. & Pu-239,240 \\
& & & \\
Sample & Dup. & Result & $+/-2$ \\
Date & No. & pCi/g & std. Dev. \\
\hline $05 / 15 / 87$ & & $0.2890 \mathrm{E}-02$ & $0.651 \mathrm{E}-03$ \\
$05 / 22 / 86$ & $0.4050 \mathrm{E}-02$ & $0.690 \mathrm{E}-03$ \\
$07 / 03 / 85$ & $0.1720 \mathrm{E}-02$ & $0.525 \mathrm{E}-03$ \\
$05 / 31 / 84$ & $0.2230 \mathrm{E}-02$ & $0.149 \mathrm{E}-02$ \\
$08 / 18 / 83$ & $0.5930 \mathrm{E}-02$ & $0.166 \mathrm{E}-02$ \\
$08 / 20 / 82$ & $0.4760 \mathrm{E}-02$ & $0.134 \mathrm{E}-02$ \\
$08 / 12 / 81$ & $0.3980 \mathrm{E}-02$ & $0.950 \mathrm{E}-03$ \\
$08 / 06 / 80$ & $0.8240 \mathrm{E}-02$ & $0.170 \mathrm{E}-02$ \\
$09 / 13 / 79$ & $0.9780 \mathrm{E}-02$ & $0.270 \mathrm{E}-02$ \\
$08 / 09 / 78$ & $0.4370 \mathrm{O}-02$ & $0.240 \mathrm{E}-02$ \\
$06 / 14 / 77$ & $0.5640 \mathrm{E}-02$ & $0.180 \mathrm{E}-02$
\end{tabular}

\begin{tabular}{llll}
$\begin{array}{l}\text { Baxter } \\
6220\end{array}$ & $\begin{array}{c}\text { Substation } \\
\text { Depth } 0-1 \text { 1n. }\end{array}$ & Pu-239,240 \\
& & & $+/-2$ \\
Sample & Dup. Result & std. Dev. \\
Date & No. & pCi/g & std \\
\hline $06 / 14 / 77$ & & $0.3350 \mathrm{E}-02$ & $0.120 \mathrm{E}-02$ \\
$09 / 25 / 74$ & & $0.3900 \mathrm{E}-02$ & $0.000 \mathrm{E}+00$
\end{tabular}

Pettett Dairy Farm

\begin{tabular}{|c|c|c|c|}
\hline $\begin{array}{l}\text { Sample } \\
\text { Date }\end{array}$ & $\begin{array}{l}\text { Dup. } \\
\text { No. }\end{array}$ & $\begin{array}{l}\text { Regult } \\
\mathrm{pCl} / \mathrm{g}\end{array}$ & $\begin{array}{l}+/{ }^{2} \\
\text { std. Dev. }\end{array}$ \\
\hline$\overline{06 / 14 / 77}$ & -- & $0.2000 \mathrm{E}-01$ & $0.330 \mathrm{E}-02$ \\
\hline
\end{tabular}

Taylar Flata No. 1

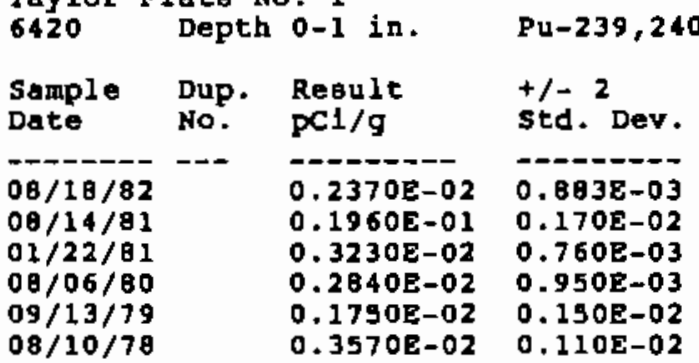

Taylor Flate No. 2

6421 Depth 0-1 in. Pu-239,240

\begin{tabular}{llll} 
Sample & Dup. & Result & $+/-2$ \\
Date & No. & pC1/g & Std. Dev. \\
\hline $05 / 15 / 87$ & & $0.1090 \mathrm{E}-01$ & $0.118 \mathrm{E}-02$ \\
$05 / 22 / 86$ & & $0.2050 \mathrm{E}-01$ & $0.331 \mathrm{E}-02$ \\
$07 / 08 / 85$ & & $0.7530 \mathrm{E}-03$ & $0.300 \mathrm{E}-03$ \\
$05 / 30 / 84$ & & $0.1350 \mathrm{E}-02$ & $0.455 \mathrm{E}-03$ \\
$08 / 26 / 83$ & & $0.3110 \mathrm{E}-01$ & $0.528 \mathrm{E}-02$ \\
$08 / 20 / 82$ & & $0.1570 \mathrm{E}-01$ & $0.316 \mathrm{E}-02$ \\
$11 / 03 / 81$ & & $0.5820 \mathrm{E}-02$ & $0.880 \mathrm{E}-03$ \\
$11 / 03 / 81$ & 2 & $0.4940 \mathrm{E}-02$ & $0.770 \mathrm{E}-03$ \\
$11 / 03 / 81$ & 1 & $0.3780 \mathrm{E}-02$ & $0.710 \mathrm{E}-03$ \\
$08 / 14 / 81$ & & $0.4250 \mathrm{E}-01$ & $0.240 \mathrm{E}-02$ \\
$01 / 22 / 81$ & & $0.1600 \mathrm{E}-01$ & $0.170 \mathrm{E}-02$ \\
$08 / 06 / 80$ & & $0.2610 \mathrm{E}-01$ & $0.350 \mathrm{E}-02$ \\
$09 / 13 / 79$ & $0.2520 \mathrm{E}-01$ & $0.260 \mathrm{E}-02$ \\
$08 / 10 / 78$ & & $<-1800 \mathrm{E}-03$ & $0.250 \mathrm{E}-02$
\end{tabular}

$\begin{array}{llll}\begin{array}{l}\text { Sagemore Farms } \\ 6358\end{array} & \begin{array}{l}\text { Depth } \\ \text { D-1 in. }\end{array} & \text { Pu-239,240 } \\ \text { Sample } & \text { Dup. } & \text { Result } & +/-2 \\ \text { Date } & \text { No. } & \text { pCi/g } & \text { Std. Dev. } \\ & - & - & - \\ 05 / 15 / 87 & & 0.1820 \mathrm{E}-02 & 0.450 \mathrm{E}-03 \\ 05 / 22 / 86 & 0.6120 \mathrm{E}-02 & 0.812 \mathrm{E}-03 \\ 07 / 08 / 85 & 0.1820 \mathrm{E}-02 & 0.524 \mathrm{E}-03 \\ 05 / 30 / 84 & 0.1890 \mathrm{E}-01 & 0.214 \mathrm{E}-02 \\ 08 / 25 / 83 & 0.7910 \mathrm{E}-02 & 0.145 \mathrm{E}-02 \\ 08 / 18 / 82 & 0.2620 \mathrm{E}-02 & 0.068 \mathrm{E}-03 \\ 11 / 02 / 81 & 0.1200 \mathrm{E}-02 & 0.570 \mathrm{E}-03 \\ 08 / 12 / 81 & 0.2770 \mathrm{E}-02 & 0.670 \mathrm{E}-03 \\ 08 / 06 / 80 & 0.7870 \mathrm{E}-02 & 0.150 \mathrm{E}-02 \\ 09 / 13 / 79 & <0.1230 \mathrm{E}-02 & 0.140 \mathrm{E}-02 \\ 08 / 09 / 78 & 0.3080 \mathrm{E}-02 & 0.220 \mathrm{E}-02 \\ 06 / 14 / 77 & 0.2100 \mathrm{E}-02 & 0.140 \mathrm{E}-02\end{array}$

\begin{tabular}{|c|c|c|c|}
\hline $\begin{array}{l}\text { agemoro } \\
\text { gis }\end{array}$ & $\begin{array}{l}\text { Farma } \\
\text { Depth }\end{array}$ & $\begin{array}{l}\text { (OC Dupl. } \\
\text { 0-1 1n. }\end{array}$ & \\
\hline & $\begin{array}{l}\text { Dup. } \\
\text { No. }\end{array}$ & & $\begin{array}{l}+/-2 \\
\text { std. Dev. }\end{array}$ \\
\hline - & $=--$ & $\begin{array}{l}0.3520 \mathrm{E}-02 \\
0.3170 \mathrm{E}-02 \\
0.1550 \mathrm{E}-02 \\
0.2120 \mathrm{E}-01\end{array}$ & $\begin{array}{l}0.793 \mathrm{E}-03 \\
0.727 \mathrm{E}-03 \\
0.509 \mathrm{E}-03 \\
0.268 \mathrm{E}-02 \\
0.101 \mathrm{E}-02 \\
0.14 \mathrm{E}-02\end{array}$ \\
\hline
\end{tabular}


TABLE B.5. (contd)

\begin{tabular}{|c|c|c|c|}
\hline $\begin{array}{l}\text { Sagemoro } \\
\text { odig }\end{array}$ & $\begin{array}{l}\text { Farmo } \\
\text { Depth }\end{array}$ & $\begin{array}{l}\text { loc Dupl. B } \\
0-1 \text { in. }\end{array}$ & $\mathrm{Pu}-239,240$ \\
\hline $\begin{array}{l}\text { Sample } \\
\text { Date }\end{array}$ & $\begin{array}{l}\text { Dup. } \\
\text { No. }\end{array}$ & $\begin{array}{l}\text { Result } \\
\mathrm{pCi} / \mathrm{g}\end{array}$ & $\begin{array}{l}+/-2 \\
\text { std. Dev. }\end{array}$ \\
\hline $\begin{array}{l}---1 / 157 \\
05 / 15 / 87 \\
05 / 22 / 86 \\
07 / 08 / 85 \\
05 / 30 / 84 \\
08 / 25 / 83 \\
08 / 18 / 82\end{array}$ & --- & 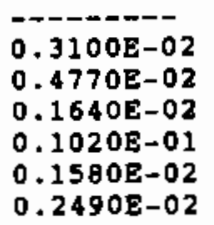 & $\begin{array}{l}0.648 \mathrm{E}-03 \\
0.725 \mathrm{E}-03 \\
0.487 \mathrm{E}-03 \\
0.205 \mathrm{E}-02 \\
0.129 \mathrm{E}-02 \\
0.101 \mathrm{E}-02\end{array}$ \\
\hline
\end{tabular}

Byerg Landing Pump Houge

\begin{tabular}{llll}
6010 & Depth $0-1$ in. & Pu-239,240 \\
Sample & Dup. & Result & $+/-2$ \\
Date & No. & pCi/g & Std. Dev. \\
\hline $09 / 25 / 74$ & & $0.2280 \mathrm{E}-01$ & $0.000 \mathrm{E}+00$ \\
$10 / 25 / 73$ & & $0.2750 \mathrm{E}-02$ & $0.000 \mathrm{E}+00$ \\
$10 / 25 / 72$ & & $0.3000 \mathrm{E}-02$ & $0.000 \mathrm{E}+00$ \\
$10 / 01 / 71$ & & $0.1200 \mathrm{E}-01$ & $0.000 \mathrm{E}+00$
\end{tabular}

Byers Landing Pump House 6033 Depth $1-2$ in.

\begin{tabular}{lll} 
Sample & Dup. & Regult \\
Date & No: & PCI/g \\
\hline $10 / 25 / 72$ & & $0.3000 \mathrm{E}-02$ \\
$10 / 01 / 71$ & & $0.6000 \mathrm{E}-03$
\end{tabular}

Byerg Landing

6011 Depth 0-1 in.

\begin{tabular}{llll} 
Sample & Dup. & $\begin{array}{l}\text { Result } \\
\text { pCi }\end{array}$ & $\begin{array}{l}+/ \mathrm{g} \\
\text { std. Dev. }\end{array}$ \\
\hline $05 / 15 / 87$ & & $0.3450 \mathrm{E}-02$ & $0.573 \mathrm{E}-03$ \\
$05 / 22 / 86$ & & $0.7900 \mathrm{E}-02$ & $0.961 \mathrm{E}-03$ \\
$06 / 19 / 85$ & $0.2740 \mathrm{E}-02$ & $0.629 \mathrm{E}-03$ \\
$05 / 30 / 84$ & $0.6590 \mathrm{E}-02$ & $0.398 \mathrm{E}-02$ \\
$08 / 25 / 83$ & $0.1160 \mathrm{E}-01$ & $0.205 \mathrm{E}-02$ \\
$08 / 18 / 82$ & $0.1510 \mathrm{E}-02$ & $0.900 \mathrm{E}-03$ \\
$08 / 12 / 81$ & $0.4920 \mathrm{E}-02$ & $0.970 \mathrm{E}-03$ \\
$08 / 06 / 80$ & & & \\
$09 / 13 / 79$ & $0.1820 \mathrm{E}-01$ & $0.270 \mathrm{E}-02$ \\
$08 / 09 / 78$ & $<0.4370 \mathrm{E}-03$ & $0.370 \mathrm{E}-02$ \\
$06 / 14 / 77$ & $0.5540 \mathrm{E}-02$ & $0.170 \mathrm{E}-02$ \\
$08 / 09 / 76$ & $0.2870 \mathrm{E}-02$ & $0.000 \mathrm{E}+00$ \\
$09 / 25 / 74$ & $0.5620 \mathrm{E}-02$ & $0.000 \mathrm{E}+00$ \\
$10 / 25 / 73$ & $0.5240 \mathrm{E}-02$ & $0.000 \mathrm{E}+00$ \\
$10 / 25 / 72$ & $0.6000 \mathrm{E}-02$ & $0.000 \mathrm{E}+00$ \\
$10 / 01 / 71$ & $0.1200 \mathrm{E}-01$ & $0.000 \mathrm{E}+00$
\end{tabular}

Pu-239, 240

$+/-2$

5td. Dev.

$0.0008+00$

$0.000 E+00$

$P u=239,240$

\begin{tabular}{|c|c|c|c|}
\hline $\begin{array}{l}\text { Yerg Lar } \\
034\end{array}$ & Depth & $1-2$ in. & $P u=239,240$ \\
\hline $\begin{array}{l}\text { Sample } \\
\text { Date }\end{array}$ & $\begin{array}{l}\text { Dup. } \\
\text { No. }\end{array}$ & $\begin{array}{l}\text { Regult } \\
\text { pci/g }\end{array}$ & $\begin{array}{l}+/{ }^{2} \\
\text { std. Dev. }\end{array}$ \\
\hline $\begin{array}{l}10 / 25 / 72 \\
10 / 01 / 71\end{array}$ & -- & $\begin{array}{l}0.1300 \mathrm{E}-01 \\
0.8000 \mathrm{E}-02\end{array}$ & $\begin{array}{l}0.000 \mathrm{E}+00 \\
0.000 \mathrm{E}+00\end{array}$ \\
\hline $\begin{array}{l}\text { Riverviev } \\
6361\end{array}$ & $\begin{array}{l}\text { (Har } \\
\text { Depth }\end{array}$ & $\begin{array}{l}\text { If Farm) } \\
0-1 \text { in. }\end{array}$ & $P u-239,24$ \\
\hline $\begin{array}{l}\text { Sample } \\
\text { Dato }\end{array}$ & $\begin{array}{l}\text { Dup. } \\
\text { No. }\end{array}$ & $\begin{array}{l}\text { Result } \\
\text { pCl/g }\end{array}$ & $\begin{array}{l}+/-2 \\
\text { std. Dev. }\end{array}$ \\
\hline $\begin{array}{l}-1-1-- \\
05 / 15 / 87 \\
05 / 22 / 86 \\
06 / 19 / 85 \\
05 / 30 / 84 \\
08 / 18 / 83 \\
08 / 18 / 82 \\
08 / 12 / 81 \\
08 / 06 / 80 \\
09 / 13 / 79 \\
08 / 09 / 78 \\
06 / 14 / 77\end{array}$ & -- & $\begin{array}{l}-0.1500 \mathrm{E}-01 \\
0.3410 \mathrm{E}-02 \\
0.5200 \mathrm{E}-02 \\
<0.1600 \mathrm{E}-02 \\
0.2080 \mathrm{E}-01 \\
0.6440 \mathrm{E}-02 \\
0.1090 \mathrm{E}-01 \\
0.1570 \mathrm{E}-01 \\
0.1670 \mathrm{E}-01 \\
0.1660 \mathrm{E}-01 \\
0.4010 \mathrm{E}-02\end{array}$ & $\begin{array}{l}-0.123 \mathrm{E}-02 \\
0.02 \\
0.636 \mathrm{E}-03 \\
0.983 \mathrm{E}-03 \\
0.180 \mathrm{E}-02 \\
0.505 \mathrm{E}-02 \\
0.166 \mathrm{E}-02 \\
0.120 \mathrm{E}-02 \\
0.180 \mathrm{E}-02 \\
0.290 \mathrm{E}-02 \\
0.340 \mathrm{E}-02 \\
0.290 \mathrm{E}-02\end{array}$ \\
\hline
\end{tabular}

Riverview Control Plot 55 6012 Depth $0-1$ in.

$P u-239,240$

\begin{tabular}{lll} 
Sample & Dup. & Regult \\
Date & No. & pC1/g \\
\hline $09 / 25 / 74$ & & $0.1680 \mathrm{E}-02$ \\
$10 / 25 / 73$ & & $0.2180 \mathrm{E}-01$ \\
$10 / 25 / 72$ & & $0.8000 \mathrm{E}-02$ \\
$10 / 01 / 71$ & & $0.1100 \mathrm{E}-01$
\end{tabular}

$+/-2$

std. Dev.

- $-1 .---1$

$0.000 E+00$

$0.000 \mathrm{E}+00$

$0.000 E+00$

$0.000 E+00$

\begin{tabular}{llll} 
Riverview Control plot s5 & \\
6039 & Depth $1-2$ in. & Pu-239,240 \\
Sample & Dup. Regult & $+/-2$ \\
Date & No. & pCi/g & Std. Dev. \\
\hdashline $10 / 25 / 72$ & & $0.9000 \mathrm{E}-02$ & $0.000 \mathrm{E}+00$ \\
$10 / 01 / 71$ & & $0.9000 \mathrm{E}-02$ & $0.000 \mathrm{E}+00$
\end{tabular}


TABLE B.5. (contd)

N Richland Control plot $\$ 6$

\begin{tabular}{llll}
6013 & Depth $0-1$ in. & Pu-239,240 \\
& & & \\
Sample & Dup. & Result & $+/-2$ \\
Date & No. & pC1/g & Std. Dev. \\
\hline $09 / 25 / 74$ & & $0.6980 E-02$ & $0.000 E+00$ \\
$10 / 25 / 73$ & & $0.1570 E-01$ & $0.100 \mathrm{E}-02$ \\
$10 / 25 / 72$ & & $0.9000 \mathrm{E}-02$ & $0.000 \mathrm{E}+00$ \\
$09 / 30 / 71$ & & $0.1400 \mathrm{E}-01$ & $0.000 \mathrm{E}+00$
\end{tabular}

N Richland control Plot 56

\begin{tabular}{llll}
6036 & Depth $1-2$ in. & Pu-239,240 \\
Sample & Dup. & Regult & $+/-2$ \\
Date & No. & pc1/g & std. Dev. \\
\hline $10 / 25 / 72$ & & $0.6000 \mathrm{E}-02$ & $0.000 \mathrm{E}+00$ \\
$09 / 30 / 71$ & & $0.2000 \mathrm{E}-02$ & $0.000 \mathrm{E}+00$
\end{tabular}

\begin{tabular}{|c|c|c|c|}
\hline $\begin{array}{l}\text { NE Corner } \\
6280\end{array}$ & $\begin{array}{l}\text { of Ex } \\
\text { Depth }\end{array}$ & $\begin{array}{c}\text { exon site } \\
0-1 \text { in. }\end{array}$ & $\mathrm{Pu}-239,240$ \\
\hline $\begin{array}{l}\text { Sample } \\
\text { Date }\end{array}$ & $\begin{array}{l}\text { Dup. } \\
\text { No. }\end{array}$ & $\begin{array}{l}\text { Result } \\
\mathrm{pCi} / \mathrm{g}\end{array}$ & $\begin{array}{l}+/-2 \\
\text { std. Dev. }\end{array}$ \\
\hline $\begin{array}{l}-08 / 09 / 76 \\
08 / 19 / 75\end{array}$ & -- & $\begin{array}{l}0.1410 \mathrm{E}-01 \\
0.1460 \mathrm{E}-01\end{array}$ & $\begin{array}{l}0.000 \mathrm{E}+00 \\
0.000 \mathrm{E}+00\end{array}$ \\
\hline
\end{tabular}

Benton City

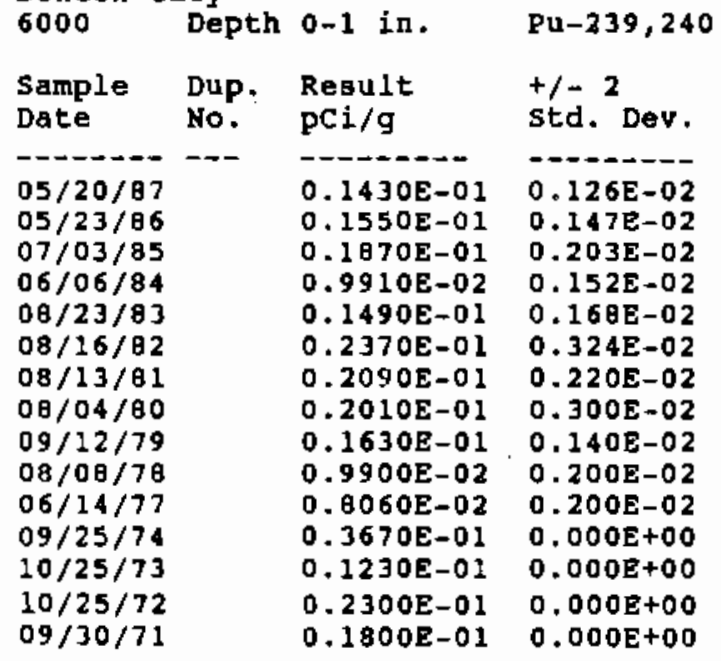

\begin{tabular}{llll}
$\begin{array}{l}\text { Benton City } \\
6023\end{array}$ & Depth & $1-2$ in. & Pu-239,240 \\
Sample & Dup. & Regult & $+/-2$ \\
Date & No. & PCi/g & std. Dev. \\
\hline$-0 / 25 / 72$ & & $0.1100 \mathrm{E}-01$ & $0.000 \mathrm{E}+00$ \\
$109 / 30 / 71$ & & $0.8000 \mathrm{E}-03$ & $0.000 \mathrm{E}+00$
\end{tabular}

Benton City (QC Dupl. A) 0B20 Depth $0-1$ in.

$\mathrm{Pu}-239,240$

\begin{tabular}{llll} 
Sample & Dup. & Result & $+/{ }^{2}$ \\
Date & No. & pCi $/ g$ & Std. Dev. \\
\hline $08 / 17 / 82$ & & $0.1290 E-01$ & $0.203 E-02$
\end{tabular}

Benton City (QC Dupl. B) 0821 Depth 0-1 in.

Pu-239,240

\begin{tabular}{llll} 
Sample & Dup. & Regult & $+/-2$ \\
Date & No. & PCi/g & Std. Dev. \\
\hdashline $08 / 17 / 82$ & & $0.5030 E-02$ & $0.126 E-02$
\end{tabular}

Sunnyoide

\begin{tabular}{llll}
6357 & Depth & $0-1$ in. & Pu-239,240 \\
Sample & Dup. & Result & $+/-2$ \\
Date & No. & pCi/g & std. Dev. \\
\hline $05 / 21 / 87$ & 1 & $0.6060 \mathrm{E}-02$ & $0.100 \mathrm{E}-02$ \\
$06 / 03 / 86$ & & $0.2120 \mathrm{E}-02$ & $0.739 \mathrm{E}-03$ \\
$06 / 17 / 85$ & & $0.1540 \mathrm{E}-01$ & $0.160 \mathrm{E}-02$ \\
$05 / 22 / 84$ & & $0.2510 \mathrm{E}-01$ & $0.259 \mathrm{E}-02$ \\
$08 / 23 / 83$ & & $0.2560 \mathrm{E}-01$ & $0.452 \mathrm{E}-02$ \\
$08 / 17 / 82$ & & $0.9160 \mathrm{E}-02$ & $0.192 \mathrm{E}-02$ \\
$08 / 13 / 81$ & $0.1260 \mathrm{E}-01$ & $0.140 \mathrm{E}-02$ \\
$08 / 04 / 80$ & & $0.1420 \mathrm{E}-01$ & $0.250 \mathrm{E}-02$ \\
$09 / 12 / 79$ & & $0.2520 \mathrm{E}-01$ & $0.350 \mathrm{E}-02$ \\
$08 / 08 / 78$ & $0.0020 \mathrm{E}-02$ & $0.290 \mathrm{E}-02$ \\
$06 / 14 / 77$ & & $0.3630 \mathrm{E}-02$ & $0.150 \mathrm{E}-02$
\end{tabular}

\begin{tabular}{llll} 
Yaking & & & \\
0512 & Depth & $0-1$ in. & Pu-239,240 \\
Sample & Dup. Regult & $+/-2$ \\
Date & No. & PCi $/ g$ & std. Dev. \\
\hdashline $05 / 21 / 87$ & 1 & $0.2100 \mathrm{E}-02$ & $0.557 \mathrm{E}-03$ \\
$05 / 21 / 87$ & & $0.3410 \mathrm{E}-02$ & $0.016 \mathrm{E}-03$ \\
$06 / 03 / 86$ & & $0.2480 \mathrm{E}-02$ & $0.865 \mathrm{E}-03$
\end{tabular}


TABLE B.5. (contd)

\begin{tabular}{llll}
$\begin{array}{l}\text { Moseg Lake } \\
\text { 0500 }\end{array}$ & Depth & & \\
& & & \\
& & in. & Pu-239,240 \\
Sample & Dup. & Result & $+/-2$ \\
Date & Ho. & pCI/g & Std. Dev. \\
\hline $05 / 08 / 87$ & 1 & $0.26308-02$ & $0.728 E-03$ \\
$05 / 08 / 87$ & & $0.2340 \mathrm{E}-02$ & $0.576 \mathrm{E}-03$ \\
$05 / 23 / 86$ & & $0.1630 \mathrm{E}-01$ & $0.124 \mathrm{E}-02$
\end{tabular}

Othello 0505

\begin{tabular}{lll} 
Sample & Dup. & Regult \\
Date & No. & pci/g \\
\hline $05 / 26 / 87$ & 1 & $0.9540 \mathrm{E}-02$ \\
$05 / 26 / 87$ & & $0.1550 \mathrm{E}-01$ \\
$05 / 02 / 86$ & & $0.4000 \mathrm{E}-02$
\end{tabular}

Connell

0503

Sample Date

05/26/87

$05 / 26 / 87$

$05 / 02 / 86$

Depth 0-1 ln.

Dup. Regult

No. pCi/g

$-2$

$0.3750 \mathrm{~B}-02$

$0.3750 \mathrm{E}-02$

$0.2710 \mathrm{E}-01$

Pu-239, 240

$+/-2$

std. Dev.

$0.129 \mathrm{E}-02$

$0.166 \mathrm{E}-02$

$0.669 \mathrm{E}-03$

Wahtucna

\begin{tabular}{llll}
0501 & Depth & $0-11 \mathrm{n}$. & Pu-239,240 \\
Sample & Dup. Result & $+/-2$ \\
Date & No. & pC1/g & std. Dev. \\
\hdashline $05 / 08 / 87$ & 1 & $0.6330 \mathrm{E}-02$ & $0.112 \mathrm{E}-02$ \\
$05 / 08 / 87$ & & $0.4910 \mathrm{E}-02$ & $0.828 \mathrm{E}-03$ \\
$05 / 23 / 86$ & & $0.2370 \mathrm{E}-01$ & $0.166 \mathrm{E}-02$
\end{tabular}

Walla Walla

\begin{tabular}{llll}
0502 & Depth & $0-1$ in. & Pu-239,240 \\
Sample & Dup. & Regult & $+/-2$ \\
Date & No. & pCilg & std. Dev. \\
\hline $05 / 29 / 87$ & & $0.7020 E-03$ & $0.324 E-03$ \\
$05 / 29 / 87$ & 1 & $0.1880 \mathrm{E}-02$ & $0.777 \mathrm{E}-03$ \\
$05 / 02 / 86$ & & $0.4710 \mathrm{E}-02$ & $0.856 \mathrm{E}-03$ \\
$11 / 15 / 85$ & & $0.1130 \mathrm{E}-01$ & $0.122 \mathrm{E}-02$
\end{tabular}

Std. Dev.

$0.670 \mathrm{E}-03$

$0.946 \mathrm{E}-03$

$0.237 \mathrm{E}-02$
McNary Dam

0504 Depth 0-1 1n. Pu-239,240

Sample Dup. Result $+/-2$

Date No. pCl/g std. Dev.

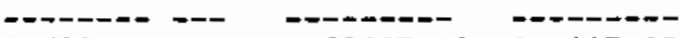

$05 / 29 / 87 \quad 0.7940 \mathrm{E}-02 \quad 0.109 \mathrm{E}-02$

$05 / 29 / 87 \quad 1 \quad 0.3580 \mathrm{E}-02 \quad 0.636 \mathrm{E}-03$

$05 / 02 / 86 \quad 0.6510 E-02 \quad 0.923 E-03$

$11 / 15 / 85 \quad 0.1530 E-01 \quad 0.235 E-02$ 
TABLE B.6. Americium-241 Concentrations in Soil at offsite Locations

\begin{tabular}{|c|c|c|c|}
\hline 6007 & Depth & $0-1$ in. & $A_{11}-241$ \\
\hline $\begin{array}{l}\text { Sample } \\
\text { Date }\end{array}$ & $\begin{array}{l}\text { Dup. } \\
\text { No. }\end{array}$ & $\begin{array}{l}\text { Result } \\
\text { PCi/g }\end{array}$ & $\begin{array}{l}+/{ }^{2} \\
\text { std. Dev. }\end{array}$ \\
\hline $06 / 14 / 77$ & $=-$ & $0.8690 \mathrm{E}-01$ & $0.670 \mathrm{E}-01$ \\
\hline
\end{tabular}

Berg Ranch

\begin{tabular}{llll}
6008 & Depth $0-1$ in. & Am-241 \\
Sample & Dup. Result & $+/ \mathbf{2}^{2}$ \\
Date & No. & pCi/g & std. Dev. \\
\hline $06 / 14 / 77$ & & $\langle 0.5180 \mathrm{E}-01$ & $0.670 \mathrm{E}-01$
\end{tabular}

\begin{tabular}{llll}
$\begin{array}{l}\text { Ringold } \\
6009\end{array}$ & Depth $0-1$ in. & Am-241 \\
Sample & Dup. & Result & $+/-2$ \\
Date & No. & pCl/g & std. Dev. \\
\hline $06 / 14 / 77$ & & $<0.3180 \mathrm{~B}-01$ & $0.670 \mathrm{E}-01$
\end{tabular}

4 End of Fir Road

\begin{tabular}{llll}
6360 & Depth $0-1$ in. & An-241 \\
Sample & Dup. Result & $+/{ }^{2}$ \\
Date & No. & pCi/g & Std. Dev. \\
\hdashline $06 / 14 / 77$ & -- & $<0.4250 \mathrm{E}-01$ & $0.660 \mathrm{E}-01$
\end{tabular}

Baxter substation

\begin{tabular}{llll}
6220 & Depth $0-1$ in. & An-241 \\
Sample & Dup. Result & $+/ 22$ \\
Date & No. & pCi/g & std. Dev. \\
\hdashline $06 / 14 / 77$ & & $<0.8090 E-02$ & $0.660 E-01$
\end{tabular}

\begin{tabular}{|c|c|c|c|}
\hline $\begin{array}{l}\text { butcelc } \\
6359\end{array}$ & $\begin{array}{l}\text { Darfy } \\
\text { Depth }\end{array}$ & $0-1$ in. & $A m-241$ \\
\hline $\begin{array}{l}\text { Sample } \\
\text { Date }\end{array}$ & $\begin{array}{l}\text { Dup. } \\
\text { No. }\end{array}$ & $\begin{array}{l}\text { Regult } \\
\text { PCi/g }\end{array}$ & $\begin{array}{l}+/=2 \\
\text { std. Dev. }\end{array}$ \\
\hline $06 / 14 / 77$ & -- & $\langle 0.2230 \mathrm{E}-01$ & $0.660 \mathrm{E}-01$ \\
\hline
\end{tabular}

\begin{tabular}{|c|c|c|c|}
\hline $\begin{array}{l}\text { Sagenore } \\
6358\end{array}$ & $\begin{array}{l}\text { Farms } \\
\text { Depth }\end{array}$ & 0-1 in. & AMr-241 \\
\hline ample & $\begin{array}{l}\text { Dup. } \\
\text { No. }\end{array}$ & $\begin{array}{l}\text { Result } \\
\mathrm{pCl} / \mathrm{g}\end{array}$ & $\begin{array}{l}+/-2 \\
\text { std. Dev. }\end{array}$ \\
\hline $\begin{array}{l}05 / 15 / 87 \\
05 / 22 / 86 \\
07 / 08 / 85 \\
05 / 30 / 84 \\
0 \theta / 18 / 82 \\
06 / 14 / 77\end{array}$ & & $\begin{array}{r}<0.78 \\
\langle 0.77 \\
\langle-.54 \\
\langle 0.41 \\
0.42 \\
<0.10\end{array}$ & $\begin{array}{l}0.169 \mathrm{E}-01 \\
0.683 \mathrm{E}-02 \\
0.306 \mathrm{E}-01 \\
0.915 \mathrm{E}-02 \\
0.292 \mathrm{E}-01 \\
0.680 \mathrm{E}-01\end{array}$ \\
\hline
\end{tabular}

\begin{tabular}{|c|c|c|c|}
\hline $\begin{array}{l}\text { Sagemore } \\
081 \mathrm{~B}\end{array}$ & $\begin{array}{l}\text { Farms } \\
\text { Depth }\end{array}$ & $\begin{array}{l}(00 \text { Dupl. A) } \\
\text { o-1 in. }\end{array}$ & Ara-241 \\
\hline $\begin{array}{l}\text { Sample } \\
\text { Date }\end{array}$ & $\begin{array}{l}\text { Dup. } \\
\text { No. }\end{array}$ & $\begin{array}{l}\text { Regult } \\
\text { PCi/g }\end{array}$ & $\begin{array}{l}+/-2 \\
\text { std. Dev. }\end{array}$ \\
\hline $\begin{array}{l}-05 / 15 / 87 \\
05 / 22 / 86 \\
05 / 08 / 85 \\
05 / 30 / 84 \\
08 / 25 / 83 \\
08 / 18 / 82\end{array}$ & --- & $\begin{array}{l}<0.5320 \mathrm{E}-02 \\
<0.1110 \mathrm{E}-02 \\
<-.47 \mathrm{BOE}-01 \\
<-.1690 \mathrm{E}-02 \\
<0.1310 \mathrm{E}-02 \\
<0.9710 \mathrm{E}-03\end{array}$ & $\begin{array}{l}0.730 \mathrm{E}-02 \\
0.711 \mathrm{E}-02 \\
0.302 \mathrm{E}-01 \\
0.372 \mathrm{E}-02 \\
0.526 \mathrm{E}-02 \\
0.291 \mathrm{E}-01\end{array}$ \\
\hline
\end{tabular}

\begin{tabular}{|c|c|c|c|}
\hline $\begin{array}{l}\text { agemore } \\
819\end{array}$ & $\begin{array}{l}\text { Farma } \\
\text { Depth }\end{array}$ & $\begin{array}{l}\text { (OC Dupl. B } \\
0-1 \text { in. }\end{array}$ & Am-241 \\
\hline $\begin{array}{l}\text { ample } \\
\text { ate }\end{array}$ & $\begin{array}{l}\text { Dup. } \\
\text { No. }\end{array}$ & $\begin{array}{l}\text { Regult } \\
\text { pCi/g }\end{array}$ & $\begin{array}{l}+/-2 \\
\text { std. Dev. }\end{array}$ \\
\hline $\begin{array}{l}05 / 15 / 87 \\
05 / 22 / 86 \\
07 / 08 / 85 \\
05 / 30 / 84 \\
08 / 25 / 83 \\
00 / 18 / 82\end{array}$ & $-\infty$ & $\begin{array}{l}<-.0450 \mathrm{E}-02 \\
<0.1060 \mathrm{E}-01 \\
<0.1010 \mathrm{E}-01 \\
<0.3220 \mathrm{E}-02 \\
<0.7380 \mathrm{E}-03 \\
<0.2580 \mathrm{E}-01\end{array}$ & $\begin{array}{l}0.980 \mathrm{E}-03 \\
0.943 \mathrm{E}-02 \\
0.13 \mathrm{BE}-01 \\
0.910 \mathrm{E}-02 \\
0.651 \mathrm{E}-02 \\
0.311 \mathrm{E}-01\end{array}$ \\
\hline
\end{tabular}


TABLE B.6. (contd)

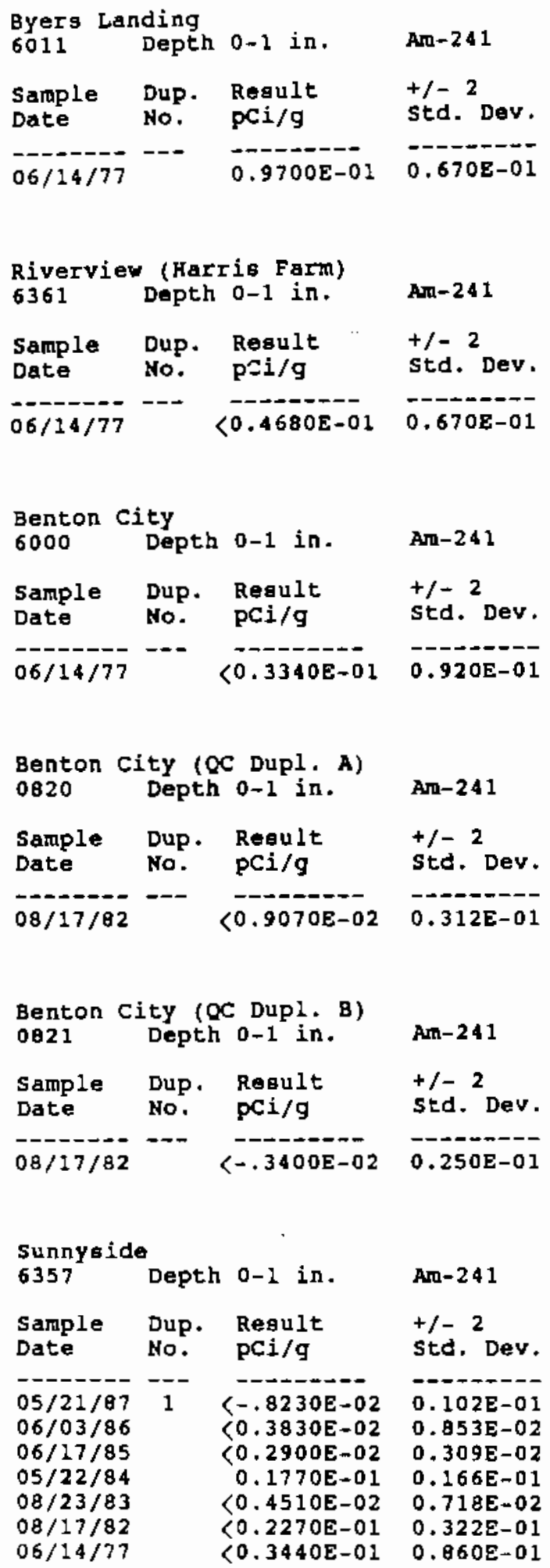


TABLE B.7. Uranium Concentrations in Soil at Offsite Locations

\begin{tabular}{|c|c|c|c|}
\hline ample & $\begin{array}{l}\text { Dup. } \\
\text { No. }\end{array}$ & $\begin{array}{l}0-1 \text { in. } \\
\text { Regult } \\
p C i / g\end{array}$ & $\begin{array}{l}\text { Uranium } \\
+/-2 \\
\text { std. Dev. }\end{array}$ \\
\hline $\begin{array}{l}--1--= \\
05 / 26 / 87 \\
05 / 02 / 86 \\
06 / 12 / 85 \\
05 / 31 / 84 \\
08 / 26 / 83 \\
08 / 30 / 82 \\
08 / 11 / 81 \\
00 / 06 / 80 \\
09 / 13 / 79 \\
08 / 09 / 78 \\
06 / 14 / 77 \\
08 / 09 / 76 \\
08 / 19 / 75 \\
09 / 23 / 74\end{array}$ & . & $\begin{array}{l}-.1670 \mathrm{E}+00 \\
0.160 \\
0.2060 \mathrm{E}+00 \\
0.3510 \mathrm{E}+00 \\
0.4340 \mathrm{E}+00 \\
0.3500 \mathrm{E}+00 \\
0.3590 \mathrm{E}+00 \\
0.4550 \mathrm{E}+00 \\
0.3350 \mathrm{E}+00 \\
0.3060 \mathrm{E}+00 \\
0.4610 \mathrm{E}+00 \\
0.1650 \mathrm{E}+00 \\
0.2760 \mathrm{E}+00 \\
0.1920 \mathrm{E}+00 \\
0.1060 \mathrm{E}+00\end{array}$ & $\begin{array}{l}0.000 \mathrm{E}+00 \\
0.000 \mathrm{D}+00 \\
0.115 \mathrm{E}+00 \\
0.117 \mathrm{E}+00 \\
0.962 \mathrm{E}-01 \\
0.12 \mathrm{~B}+00 \\
0.160 \mathrm{E}+00 \\
0.120 \mathrm{E}+00 \\
0.110 \mathrm{E}+00 \\
0.150 \mathrm{E}+00 \\
0.600 \mathrm{E}-01 \\
0.000 \mathrm{E}+00 \\
0.000 \mathrm{E}+00 \\
0.000 \mathrm{E}+00\end{array}$ \\
\hline $10 / 25 / 73$ & & $0.5340 \mathrm{E}+00$ & $0.000 E+00$ \\
\hline
\end{tabular}

Berg Ranch

\begin{tabular}{|c|c|c|c|}
\hline $\begin{array}{l}\text { ample } \\
\text { ate }\end{array}$ & $\begin{array}{l}\text { Dup. } \\
\text { No. }\end{array}$ & $\begin{array}{l}0-1 \text { in. } \\
\text { Regult } \\
\text { pci/g }\end{array}$ & $\begin{array}{l}\text { Uranium } \\
+/-2 \\
\text { std. Dev. }\end{array}$ \\
\hline $\begin{array}{l}05 / 26 / 87 \\
05 / 02 / 86 \\
06 / 26 / 85 \\
05 / 30 / 84 \\
08 / 26 / 83 \\
08 / 20 / 82 \\
08 / 11 / 81 \\
08 / 11 / 81 \\
08 / 06 / 80 \\
09 / 13 / 79 \\
08 / 09 / 78 \\
06 / 14 / 77 \\
08 / 09 / 76 \\
08 / 19 / 75\end{array}$ & $--\infty$ & 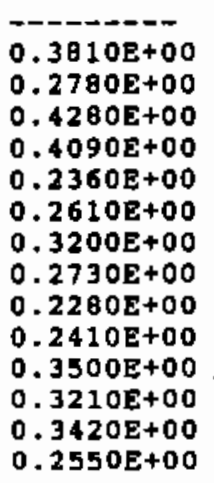 & $\begin{array}{l}0.000 \mathrm{E}+00 \\
0.000 \\
0.000 \mathrm{E}+00 \\
0.142 \mathrm{E}+00 \\
0.110 \mathrm{E}+00 \\
0.662 \mathrm{E}-01 \\
0.913 \mathrm{E}-01 \\
0.110 \mathrm{E}+00 \\
0.950 \mathrm{E}-01 \\
0.800 \mathrm{E}-01 \\
0.840 \mathrm{E}-01 \\
0.150 \mathrm{E}+00 \\
0.680 \mathrm{E}-01 \\
0.000 \mathrm{E}+00 \\
0.000 \mathrm{E}+00\end{array}$ \\
\hline & & $\begin{array}{l}0 E-01 \\
0 E+00\end{array}$ & $\begin{array}{l}0.000 \mathrm{E}+00 \\
0.000 \mathrm{E}+00\end{array}$ \\
\hline
\end{tabular}

Cook Bros. Dairy Farm

\begin{tabular}{llll}
6219 & Depth $0-1$ in. & Uranium \\
Sample & Dup. & Regult & $+/-2$ \\
Date & No. & pCi/g & std. Dev, \\
\hdashline $09 / 24 / 74$ & & $0.3910 E+00$ & $0.000 E+00$
\end{tabular}

Ringold

6009 Depth $0-1$ in. Uranium

\begin{tabular}{|c|c|c|c|}
\hline $\begin{array}{l}\text { Sample } \\
\text { Date }\end{array}$ & $\begin{array}{l}\text { Dup. } \\
\text { No. }\end{array}$ & $\begin{array}{l}\text { Result } \\
\text { pct/g }\end{array}$ & $\begin{array}{l}+/-2 \\
\text { std. Dev. }\end{array}$ \\
\hline $\begin{array}{l}------\bar{l} \\
05 / 15 / 87 \\
05 / 22 / 86 \\
07 / 00 / 85 \\
05 / 30 / 84 \\
08 / 18 / 83 \\
08 / 20 / 82 \\
08 / 12 / 81 \\
08 / 06 / 80 \\
09 / 13 / 79 \\
08 / 09 / 78 \\
06 / 14 / 77 \\
08 / 09 / 76 \\
08 / 19 / 75 \\
10 / 25 / 73\end{array}$ & $--\pi$ & $\begin{array}{l}\overline{0} .6650 \mathrm{E}+00 \\
0.1140 \mathrm{E}+01 \\
0.6420 \mathrm{E}+00 \\
0.7800 \mathrm{E}+00 \\
0.3720 \mathrm{E}+00 \\
0.4330 \mathrm{E}+00 \\
0.8210 \mathrm{E}+00 \\
0.3360 \mathrm{E}+00 \\
0.1200 \mathrm{E}+01 \\
0.6630 \mathrm{E}+00 \\
0.2060 \mathrm{E}+00 \\
0.2970 \mathrm{E}+00 \\
0.2080 \mathrm{E}+00 \\
0.5040 \mathrm{E}+00\end{array}$ & $\begin{array}{l}-.000 \mathrm{E}+00 \\
0.000 \mathrm{E}+00 \\
0.000 \mathrm{E}+00 \\
0.256 \mathrm{E}+00 \\
0.206 \mathrm{E}+00 \\
0.101 \mathrm{E}+00 \\
0.152 \mathrm{E}+00 \\
0.290 \mathrm{E}+00 \\
0.120 \mathrm{E}+00 \\
0.420 \mathrm{E}+00 \\
0.150 \mathrm{E}+00 \\
0.680 \mathrm{E}-01 \\
0.000 \mathrm{E}+00 \\
0.000 \mathrm{E}+00 \\
0.000 \mathrm{E}+00\end{array}$ \\
\hline
\end{tabular}

F End of Fir Road

6360 Depth 0-1 in. Uranium

\begin{tabular}{llll} 
Sample & Dup, & Result & $+/-2$ \\
Date & No. & pCi/g & Std. Dev. \\
\hline $05 / 15 / 87$ & & $0.5530 \mathrm{E}+00$ & $0.000 \mathrm{E}+00$ \\
$05 / 22 / 86$ & & $0.3240 \mathrm{E}+00$ & $0.000 \mathrm{D}+00$ \\
$07 / 03 / 85$ & $0.7250 \mathrm{E}+00$ & $0.222 \mathrm{E}+00$ \\
$05 / 31 / 84$ & $0.5360 \mathrm{E}+00$ & $0.140 \mathrm{E}+00$ \\
$08 / 18 / 63$ & $0.4710 \mathrm{E}+00$ & $0.128 \mathrm{E}+00$ \\
$08 / 20 / 82$ & $0.2750 \mathrm{E}+00$ & $0.962 \mathrm{E}-01$ \\
$08 / 12 / 81$ & $0.8630 \mathrm{E}+00$ & $0.310 \mathrm{E}+00$ \\
$08 / 06 / 80$ & $0.4590 \mathrm{E}+00$ & $0.160 \mathrm{E}+00$ \\
$09 / 13 / 79$ & $0.5660 \mathrm{E}+00$ & $0.200 \mathrm{E}+00$ \\
$08 / 09 / 78$ & $0.3500 \mathrm{E}+00$ & $0.150 \mathrm{E}+00$ \\
$06 / 14 / 77$ & $0.3250 \mathrm{E}+00$ & $0.600 \mathrm{E}-01$
\end{tabular}

Baxter subetation

6220 Depth $0-1$ in. Uranium

\begin{tabular}{llll} 
Sample & Dup. & Regult & $+/-2$ \\
Date & No. & pCi/g & Std. Dev. \\
\hline $06 / 14 / 77$ & & $0.3180 \mathrm{E}+00$ & $0.680 \mathrm{E}-01$ \\
$09 / 24 / 74$ & & $0.3610 \mathrm{E}+00$ & $0.000 E+00$
\end{tabular}

Pettett Dairy Farn

6359 Depth $0-1$ in. Uranium

\begin{tabular}{llll} 
Sample & Dup. & Regult & $+/-2$ \\
Date & No. & pCi/g & std. Dev. \\
\hline $06 / 14 / 77$ & & $0.4700 E+00$ & $0.6808-01$
\end{tabular}




\section{TABLE B.7. (contd)}

Taylor Flata No. 1

\begin{tabular}{|c|c|c|c|}
\hline 420 & Depth & 0-1 in. & Uranium \\
\hline $\begin{array}{l}\text { Sample } \\
\text { Date }\end{array}$ & $\begin{array}{l}\text { Dup. } \\
\text { No. }\end{array}$ & $\begin{array}{l}\text { Result } \\
\mathrm{pCi} / \mathrm{g}\end{array}$ & $\begin{array}{l}+/-2 \\
\text { std. Dev. }\end{array}$ \\
\hline $\begin{array}{l}08 / 18 / 82 \\
00 / 14 / 81 \\
01 / 22 / 81 \\
08 / 06 / 80 \\
09 / 13 / 79 \\
08 / 20 / 78\end{array}$ & --- & $\begin{array}{l}0.7410 \mathrm{E}+00 \\
0.1140 \mathrm{E}+01 \\
0.2720 \mathrm{E}+00 \\
0.1420 \mathrm{E}+01 \\
0.1130 \mathrm{E}+01 \\
0.1150 \mathrm{E}+01\end{array}$ & $\begin{array}{l}0.249 \mathrm{E}+00 \\
0.400 \mathrm{E}+00 \\
0.950 \mathrm{E}-01 \\
0.500 \mathrm{E}+00 \\
0.400 \mathrm{E}+00 \\
0.150 \mathrm{E}+00\end{array}$ \\
\hline
\end{tabular}

Taylor flats Ho. 2

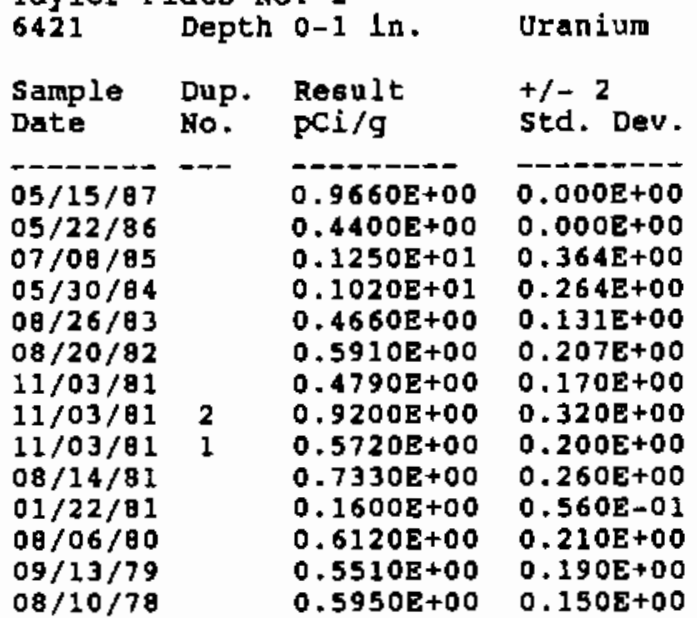

Sagemore Farms

6350

Depth $0-1$ in.

Sample

Date

Dup. Regult

No. $\mathrm{pCi} / \mathrm{g}$

$05 / 15 / 87$

$05 / 22 / 86$

$07 / 08 / 85$

$05 / 30 / 84$

$00 / 25 / \theta 3$

$08 / 18 / 62$

$11 / 02 / 81$

$08 / 12 / 81$

$00 / 06 / 80$

$09 / 13 / 79$

$08 / 09 / 78$

$06 / 14 / 77$

$\mathrm{PCi} / \mathrm{g}$

$0.2370 \mathrm{E}+00$

$0.3100 \mathrm{E}+00$

$0.5760 \mathrm{E}+00$

$0.5030 \mathrm{E}+00$

$0.3760 E+00$

$0.3090 \mathrm{E}+00$

$0.5940 \mathrm{E}+00$

$0.6330 E+00$

$0.3680 E+00$

$0.4130 E+00$

$0.3000 E+00$

$0.2400 \mathrm{E}+00$
Uranium

$+/-2$

std. Dev.

$0.000 E+00$

$0.000 E+00$

$0.180 \mathrm{E}+00$

$0.132 \mathrm{E}+00$

$0.105 \mathrm{E}+00$

$0.108 \mathrm{E}+00$

$0.210 E+00$

$0.220 \mathrm{E}+00$

$0.1308+00$

$0.1408+00$

$0.150 \mathrm{E}+00$

$0.680 \mathrm{E}-01$
Sagenore Farme (QC Dupl. A)

ob18 Depth 0-1 in. Uranium

\begin{tabular}{llll} 
Sample & Dup. & Result & $+/-2$ \\
Date & No. & pCi/g & std. Dev. \\
\hline $05 / 15 / 87$ & & $0.4130 \mathrm{E}+00$ & $\mathbf{0 . 0 0 0 E + 0 0}$ \\
$05 / 22 / 86$ & & $0.4140 \mathrm{E}+00$ & $0.000 \mathrm{E}+00$ \\
$07 / 08 / 83$ & & $0.3370 \mathrm{E}+00$ & $0.111 \mathrm{E}+00$ \\
$05 / 30 / 84$ & & $0.4270 \mathrm{E}+00$ & $0.114 \mathrm{E}+00$ \\
$08 / 25 / 83$ & & $0.4410 \mathrm{E}+00$ & $0.123 \mathrm{E}+00$ \\
$08 / 18 / 82$ & & $0.3290 \mathrm{~B}+00$ & $0.115 \mathrm{~B}+00$
\end{tabular}

Sagenore Farmi (OC Dupl. B)

0019 Depth 0-1 in. Uranium

\begin{tabular}{|c|c|c|c|}
\hline $\begin{array}{l}\text { ample } \\
\text { ate }\end{array}$ & $\begin{array}{l}\text { Dup. } \\
\text { No. }\end{array}$ & $\begin{array}{l}\text { Result } \\
\text { pCi/g }\end{array}$ & $\begin{array}{l}+/-2 \\
\text { std. Dev. }\end{array}$ \\
\hline $\begin{array}{l}05 / 15 / 87 \\
05 / 22 / 86 \\
07 / 08 / 85 \\
05 / 30 / 84 \\
00 / 25 / 83 \\
00 / 18 / 82\end{array}$ & --- & $\begin{array}{l}- \\
0.3630 E+00 \\
0.3920 E+00 \\
0.6030 E+00 \\
0.9120 E+00 \\
0.3500 E+00 \\
0.2490 E+00\end{array}$ & $\begin{array}{l}0.000 \mathrm{E}+00 \\
0.000 \mathrm{E}+00 \\
0.189 \mathrm{E}+00 \\
0.224 \mathrm{E}+00 \\
0.974 \mathrm{E}-01 \\
0.070 \mathrm{E}-01\end{array}$ \\
\hline
\end{tabular}

Byera Landing Pump Houge

6010 Depth $0-1$ in.

Uranium

\begin{tabular}{llll} 
Sample & Dup. & Regu1t & $+/-2$ \\
Date & No. & pCi/g & Std. Dev. \\
\hline $09 / 24 / 74$ & & $0.6090 \mathrm{E}+00$ & $0.000 \mathrm{E}+00$ \\
$10 / 25 / 73$ & & $0.2790 \mathrm{E}+00$ & $0.000 \mathrm{E}+00$
\end{tabular}

Byers Landing

6011 Depth $0-1$ in. Uraniur

Sample Dup. Regult $+/-2$

Date No. pCl/g std. Dev.

$\overline{05 / 15 / 87}-\overline{0.9680 \mathrm{E}-01} 0 . \overline{0.000 \mathrm{E}+00}$

$05 / 22 / 86 \quad 0.2590 E+00 \quad 0.000 E+00$

$06 / 19 / 85 \quad 0.3940 E+00 \quad 0.129 E+00$

$05 / 30 / 84 \quad 0.4270 \mathrm{E}+00 \quad 0.113 \mathrm{E}+00$

$08 / 25 / 83 \quad 0.3220 \mathrm{E}+00 \quad 0.904 \mathrm{E}-01$

$08 / 18 / 82 \quad 0.5460 E+00 \quad 0.191 E+00$

$0 \theta / 12 / 81 \quad 0.4710 E+00 \quad 0.170 E+00$

$00 / 06 / 80 \quad 0.3410 E+00 \quad 0.120 E+00$

$09 / 13 / 79 \quad 0.4510 E-01 \quad 0.230 E-01$

$08 / 09 / 7 \theta \quad 0.3540 E+00 \quad 0.150 E+00$

$06 / 14 / 77 \quad 0.2780 E+00 \quad 0.680 \mathrm{E}-01$

$08 / 09 / 76 \quad 0.4050 \mathrm{E}+00 \quad 0.000 \mathrm{E}+00$

$09 / 24 / 74 \quad 0.3900 E+00 \quad 0.000 E+00$

$10 / 25 / 73 \quad 0.1770 E+00 \quad 0.000 E+00$ 


\section{TABLE B. 7 . (contd)}

\begin{tabular}{|c|c|c|c|}
\hline $\begin{array}{l}\text { Rivervie } \\
6361\end{array}$ & $\begin{array}{r}\text { (Ha } \\
\text { Dept }\end{array}$ & $\begin{array}{l}18 \text { Farm) } \\
0-1 \text { in. }\end{array}$ & \\
\hline & $\begin{array}{l}\text { Dup. } \\
\text { No. }\end{array}$ & $\begin{array}{l}\text { Regult } \\
\text { pci/g }\end{array}$ & $\begin{array}{l}+/-2 \\
\text { std. Dev. }\end{array}$ \\
\hline $\begin{array}{l}-05 / 15 / 87 \\
05 / 22 / 86 \\
06 / 19 / 85 \\
05 / 30 / 84 \\
08 / 18 / 83 \\
08 / 18 / 82 \\
08 / 12 / 81 \\
08 / 06 / 80 \\
09 / 13 / 79 \\
08 / 09 / 78 \\
06 / 14 / 77\end{array}$ & - - & $\begin{array}{l}\overline{0} .2960 \mathrm{E}+00 \\
0.2560 \mathrm{E}+00 \\
0.4400 \mathrm{E}+00 \\
0.3210 \mathrm{E}+00 \\
0.3730 \mathrm{E}+00 \\
0.1400 \mathrm{E}+00 \\
0.6390 \mathrm{E}+00 \\
0.3250 \mathrm{E}+00 \\
0.4370 \mathrm{E}+00 \\
0.2730 \mathrm{E}+00 \\
0.2890 \mathrm{E}+00\end{array}$ & $\begin{array}{l}-.-1- \\
0.000 \mathrm{E}+00 \\
0.000 \mathrm{E}+00 \\
0.142 \mathrm{E}+00 \\
0.850 \mathrm{E}-01 \\
0.102 \mathrm{E}+00 \\
0.492 \mathrm{E}-01 \\
0.230 \mathrm{E}+00 \\
0.110 \mathrm{E}+00 \\
0.150 \mathrm{E}+00 \\
0.150 \mathrm{E}+00 \\
0.680 \mathrm{E}-01\end{array}$ \\
\hline
\end{tabular}

Riverview Control Plot 55

\begin{tabular}{llll}
6012 & Depth $0-11 \mathrm{l}$. & Uranium \\
Sample & Dup. Result & $+/-2$ \\
Date & No. & pCl/g & std. Dev. \\
\hdashline $09 / 24 / 74$ & & $0.4220 \mathrm{E}+00$ & $0.000 \mathrm{E}+00$ \\
$10 / 25 / 73$ & & $0.1410 \mathrm{E}+00$ & $0.000 \mathrm{E}+00$
\end{tabular}

N Richland Control Plot 56

\begin{tabular}{llll}
6013 & Depth $0-1$ in. & Uranium \\
Sample & Dup. Result & $+/-2$ \\
Date & No. & pCi/g & Std. Dev. \\
\hdashline $09 / 24 / 74$ & & $0.2550 E+00$ & $0.000 E+00$ \\
$10 / 25 / 73$ & & $0.3460 \mathrm{E}+00$ & $0.000 \mathrm{E}+00$
\end{tabular}

\begin{tabular}{llll} 
NE Corner of Exxon Site & \\
6280 & Depth $0-1$ in. & Uranium \\
Sample & Dup. & Regult & $+/-2$ \\
Date & No. & pCi $/ g$ & Std. Dev. \\
\hline $08 / 09 / 76$ & & $0.2510 \mathrm{E}+00$ & $0.000 \mathrm{D}+00$ \\
$0 \mathrm{~B} / 19 / 75$ & & $0.1400 \mathrm{E}+00$ & $0.000 \mathrm{E}+00$
\end{tabular}

\begin{tabular}{llll}
$\begin{array}{l}\text { Benton City } \\
6000\end{array}$ & & \\
& Depth & $0-1$ in. & Uraniur \\
Sample & Dup. & Regult & $+/-2$ \\
Date & No. & pCi/g & std. Dov. \\
\hline $05 / 20 / 87$ & & $0.3930 E+00$ & $0.000 E+00$ \\
$05 / 23 / 86$ & $0.4490 E+00$ & $0.000 E+00$ \\
$07 / 03 / 85$ & $0.6390 E+00$ & $0.200 E+00$ \\
$06 / 06 / 84$ & $0.9140 E+00$ & $0.244 E+00$ \\
$08 / 23 / 83$ & $0.4390 E+00$ & $0.122 E+00$ \\
$08 / 16 / 82$ & $0.5550 E+00$ & $0.194 E+00$ \\
$08 / 13 / 81$ & $0.6260 E+00$ & $0.220 E+00$ \\
$08 / 04 / 80$ & $0.6970 E+00$ & $0.240 E+00$ \\
$09 / 12 / 79$ & $0.4430 E+00$ & $0.160 E+00$ \\
$08 / 08 / 78$ & $0.5000 E+00$ & $0.150 E+00$ \\
$06 / 14 / 77$ & $0.3260 E+00$ & $0.680 E-01$ \\
$09 / 24 / 74$ & $0.2920 E+00$ & $0.000 E+00$ \\
$10 / 25 / 73$ & $0.6700 E+00$ & $0.000 E+00$
\end{tabular}

\begin{tabular}{llll}
$\begin{array}{l}\text { Benton City (OC Dupl. B) } \\
\text { 0821 }\end{array}$ & Depth 0-1 in. & Uranium \\
Sample & Dup. Result & $+/ 2$ \\
Date & No. & pCl/g & std, Dev. \\
\hline $08 / 17 / 82$ & & $0.1970 \mathrm{E}+00$ & $0.690 \mathrm{E}-01$
\end{tabular}

sunnyside

\begin{tabular}{llll}
6357 & Depth & $0-1$ in. & Uranium \\
Sample & Dup. & Regult & $+/-2$ \\
Date & No. & pCi/g & std. Dev. \\
\hline $05 / 21 / 87$ & I & $0.2860 E+00$ & $0.000 \mathrm{E}+00$ \\
$06 / 03 / 86$ & & $0.3070 \mathrm{E}+00$ & $0.000 \mathrm{E}+00$ \\
$06 / 17 / 85$ & & $0.2580 \mathrm{E}+00$ & $0.89 \mathrm{BE}-01$ \\
$05 / 22 / 84$ & & $0.2580 \mathrm{E}+00$ & $0.706 \mathrm{E}-01$ \\
$08 / 23 / 83$ & & $0.1960 \mathrm{E}+00$ & $0.54 \mathrm{BE}-01$ \\
$08 / 17 / 82$ & & $0.1720 \mathrm{E}+00$ & $0.602 \mathrm{E}-01$ \\
$08 / 13 / 81$ & & $0.3360 \mathrm{E}+00$ & $0.120 \mathrm{E}+00$ \\
$08 / 04 / 80$ & & $0.4090 \mathrm{E}+00$ & $0.140 \mathrm{E}+00$ \\
$09 / 13 / 79$ & & $0.2230 \mathrm{E}+00$ & $0.780 \mathrm{E}-01$ \\
$08 / 08 / 78$ & & $0.3840 \mathrm{E}+00$ & $0.150 \mathrm{E}+00$ \\
$06 / 14 / 77$ & & $0.4710 \mathrm{E}+00$ & $0.680 \mathrm{E}-01$
\end{tabular}




\section{TABLE B. 7 . (contd)}

\begin{tabular}{|c|c|c|c|}
\hline $\begin{array}{l}\text { Yakima } \\
0512 \\
\text { Sanple } \\
\text { Date }\end{array}$ & $\begin{array}{l}\text { Depth } \\
\text { Dup. } \\
\text { no. }\end{array}$ & $\begin{array}{l}\text { o-l in. } \\
\text { Result } \\
\text { pci/g }\end{array}$ & $\begin{array}{l}\text { Uranium } \\
+/-2 \\
\text { std. Dev. }\end{array}$ \\
\hline $\begin{array}{l}05 / 21 / 87 \\
05 / 21 / 87 \\
06 / 03 / 86\end{array}$ & --- & $\begin{array}{l}0.4190 \mathrm{E}+00 \\
0.3970 \mathrm{E}+00 \\
0.4780 \mathrm{E}+00\end{array}$ & $\begin{array}{l}0.000 \mathrm{E}+00 \\
0.000 \mathrm{E}+00 \\
0.000 \mathrm{E}+00\end{array}$ \\
\hline $\begin{array}{l}\text { Moseg Lak } \\
0500\end{array}$ & kepth & $0-1$ in. & Uranium \\
\hline $\begin{array}{l}\text { Sample } \\
\text { Date }\end{array}$ & $\begin{array}{l}\text { Dup. } \\
\text { No. }\end{array}$ & $\begin{array}{l}\text { Regult } \\
\text { pCi/g }\end{array}$ & $\begin{array}{l}+/-2 \\
\text { std. Dev }\end{array}$ \\
\hline $\begin{array}{l}05 / 08 / 87 \\
05 / 08 / 87 \\
05 / 23 / 86\end{array}$ & --- & $\begin{array}{l}0.2940 \mathrm{E}+00 \\
0.8160 \mathrm{E}-01 \\
0.1120 \mathrm{E}+00\end{array}$ & $\begin{array}{l}0.000 E+00 \\
0.000 E+00 \\
0.000 E+00\end{array}$ \\
\hline
\end{tabular}

Walla Walla

0502 Depth 0-1 in. Uranium

\begin{tabular}{|c|c|c|c|}
\hline $\begin{array}{l}\text { Sample } \\
\text { Date }\end{array}$ & $\begin{array}{l}\text { Dup. } \\
\text { No. }\end{array}$ & $\begin{array}{l}\text { Regult } \\
\text { pCi/g }\end{array}$ & $\begin{array}{l}+/-2 \\
\text { std. Dev. }\end{array}$ \\
\hline $\begin{array}{l}-05 / 29 / 87 \\
05 / 29 / 87 \\
05 / 02 / 86 \\
11 / 15 / 85\end{array}$ & -- & $\begin{array}{l}0.5160 \mathrm{E}+00 \\
0.7220 \mathrm{E}+00 \\
0.3480 \mathrm{E}+00 \\
0.1980 \mathrm{E}+00\end{array}$ & $\begin{array}{l}0.000 \mathrm{E}+00 \\
0.000 \mathrm{E}+00 \\
0.000 \mathrm{E}+00 \\
0.714 \mathrm{E}-01\end{array}$ \\
\hline
\end{tabular}

\begin{tabular}{llll}
$\begin{array}{l}\text { McNary } \\
\text { O504 }\end{array}$ & $\begin{array}{l}\text { Depth } \\
0-1 \text { in. }\end{array}$ & Uranium \\
Sample & Dup. & Regult & $+/-2$ \\
Date & No. & pCi/g & std. Dev. \\
\hline $05 / 29 / 87$ & & $0.3690 \mathrm{E}+00$ & $0.000 \mathrm{E}+00$ \\
$05 / 29 / 87$ & 1 & $0.2610 \mathrm{E}+00$ & $0.000 \mathrm{E}+00$ \\
$05 / 02 / 86$ & & $0.4130 \mathrm{E}+00$ & $0.000 \mathrm{E}+00$ \\
$11 / 15 / 85$ & & $0.1470 \mathrm{E}+00$ & $0.550 \mathrm{E}-01$
\end{tabular}

Othello

\begin{tabular}{llll}
0505 & Depth $0-1$ in. & Uranium \\
Sample & Dup. & Result & $+/-2$ \\
Date & No. & pCl/g & Std. Dev. \\
\hline $05 / 26 / 87$ & 1 & $0.2220 E+00$ & $0.000 E+00$ \\
$05 / 26 / 87$ & & $0.2510 E+00$ & $0.000 E+00$ \\
$05 / 02 / 86$ & & $0.2000 E+00$ & $0.000 E+00$
\end{tabular}

\begin{tabular}{llll}
$\begin{array}{l}\text { Connell } \\
0503\end{array}$ & Depth $0-1$ in. & Uranium \\
Sample & Dup. & Result & $+/-2$ \\
Date & No. & pCi/g & std. Dev. \\
\hline $05 / 26 / 87$ & 1 & $0.30108+00$ & $0.000 E+00$ \\
$05 / 26 / 87$ & & $0.3440 E+00$ & $0.000 E+00$ \\
$05 / 02 / 86$ & & $0.2490 E+00$ & $0.000 E+00$
\end{tabular}

\begin{tabular}{|c|c|c|c|}
\hline 0501 & Depth & $0-1$ in. & Uranium \\
\hline $\begin{array}{l}\text { Sample } \\
\text { Date }\end{array}$ & $\begin{array}{l}\text { Dup. } \\
\text { No. }\end{array}$ & $\begin{array}{l}\text { Reeult } \\
\mathrm{pCi} / \mathrm{g}\end{array}$ & $\begin{array}{l}+/-2 \\
\text { std. Dev. }\end{array}$ \\
\hline $\begin{array}{l}----1--7 \\
05 / 08 / 87 \\
05 / 08 / 87 \\
05 / 23 / 86\end{array}$ & $--=$ & $\begin{array}{l}0.2300 E+00 \\
0.2550 E+00 \\
0.1900 E+00\end{array}$ & $\begin{array}{l}0.000 E+00 \\
0.000 E+00 \\
0.000 E+00\end{array}$ \\
\hline
\end{tabular}





\section{APPENDIX C}

UNPUBLISHED RESULTS FROM SPECIAL-PURPOSE STUDIES 


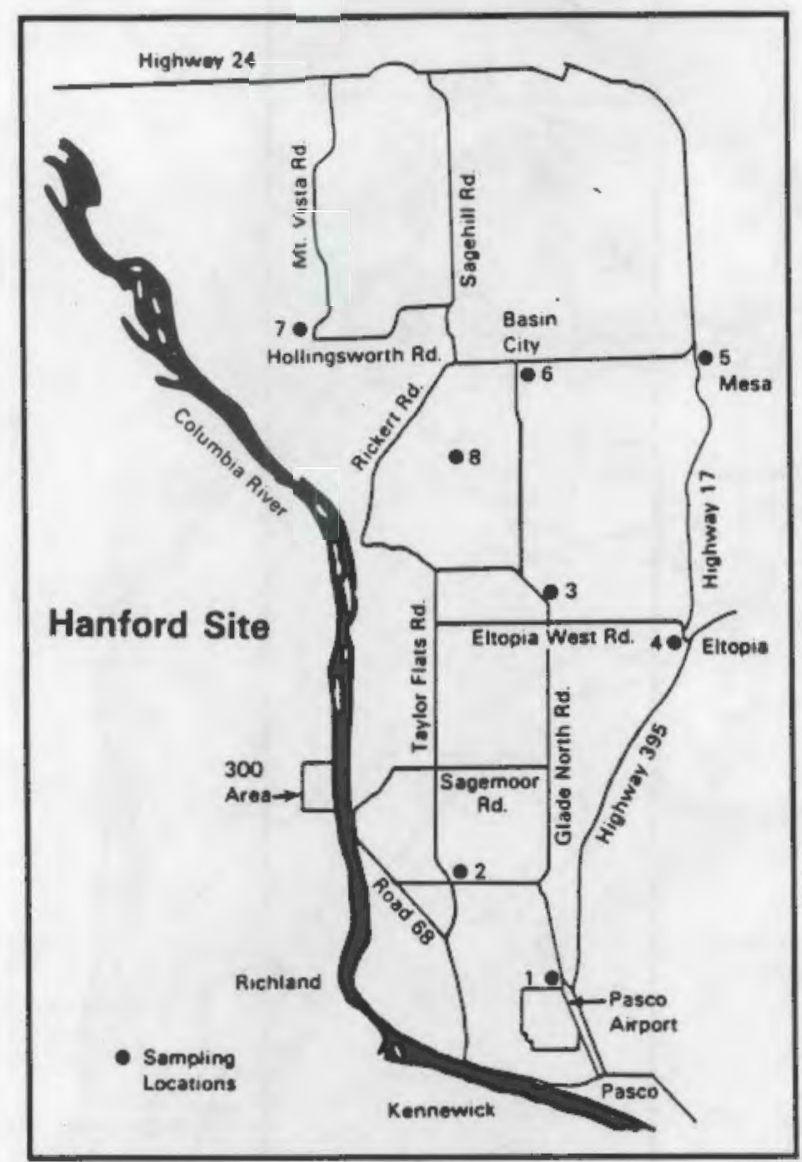

1987 Special Duplicate Soll Samples - Downwind of the Hanford Site

\begin{tabular}{|c|c|c|c|c|c|c|}
\hline \multirow[b]{2}{*}{ Location } & \multirow{3}{*}{$\begin{array}{l}\text { Map } \\
\text { o. }\end{array}$} & \\
\hline & & $5 r-90$ & $c s-137$ & $P u-238$ & $\mathrm{Pu}-239 / 40$ & Uranium \\
\hline N. Pasco & & $0.19 \pm 0.05$ & $0.76 \div 0.09$ & $0.0003 \pm 0.0002$ & $0.014 \pm 0.002$ & $0.14 \pm 0.05$ \\
\hline Taylor Flats Rd & 2 & 0 & $1.07 \pm$ & $1007 \pm$ & $\begin{array}{l}0.017 \\
0.021\end{array}$ & $\begin{array}{l}0.24 \pm 0.08 \\
0.26 \pm 0.08\end{array}$ \\
\hline & & $0.30 \pm 0 .($ & $002=0$ & $0.0006 \mp 0$. & $0.016 \pm$ & $0.20 \pm 0$. \\
\hline Merrills Corner & 3 & $0.16 \pm 0$. & $1.02 \pm 0.12$ & $0.0010 \pm 0$. & $0.020 \pm$ & $0.31 \pm 0.10$ \\
\hline & & $0.19 \pm 0.05$ & $1.26 \pm 0.14$ & & $0.022 \pm 0.0$ & \\
\hline El topia & 4 & $\begin{array}{l}0.28 \pm 0.07 \\
0.33 \pm 0.08\end{array}$ & $\begin{array}{l}0.90 \pm 0.11 \\
0.78 \pm 0.09\end{array}$ & $\begin{array}{l}0.0005 \pm 0.0 \\
0.0006 \pm 0.0\end{array}$ & $\begin{array}{l}0.016 \pm 0.002 \\
0.019 \pm 0.003\end{array}$ & $\begin{array}{l}0.22 \pm 0.07 \\
0.25 \pm 0.08\end{array}$ \\
\hline Mesa & 5 & $0.34 \pm 0$ & \pm 0.15 & \pm \pm 0 & & 0.12 \\
\hline Basin $C$ & & $33 \pm 0$ & & & & \\
\hline Basin Cfty & 6 & $\begin{array}{l}0.26 \pm 0 . \\
0.29 \pm 0 .\end{array}$ & $\begin{array}{l}0.85 \div 0.10 \\
0.94+0.11\end{array}$ & 0.0006 & $0.017 \pm 0.002$ & 0.17 \\
\hline Hollingsworth Rd & 7 & $0.16 \pm 0$. & $3 \pm 0.07$ & & & $0.18 \div 0.06$ \\
\hline & & & $0.53 \pm 0.07$ & & $0.009 \pm 0.001$ & $0.15 \mp 0.05$ \\
\hline Ringold & 8 & $0.16 \pm 0.04$ & $0.60 \pm 0.08$ & $05 \pm 0.0003$ & $0.012 \pm 0.002$ & $0.42 \pm 0.13$ \\
\hline & & $0.18 \pm 0.04$ & $0.77 \pm 0.09$ & $8 \pm 0.0003$ & $0.016 \pm 0.002$ & $0.43 \pm 0.13$ \\
\hline
\end{tabular}

FIGURE C.T. Unpublished Results from a Special Purpose Study Downwind of the Hanford Site, May 1987 


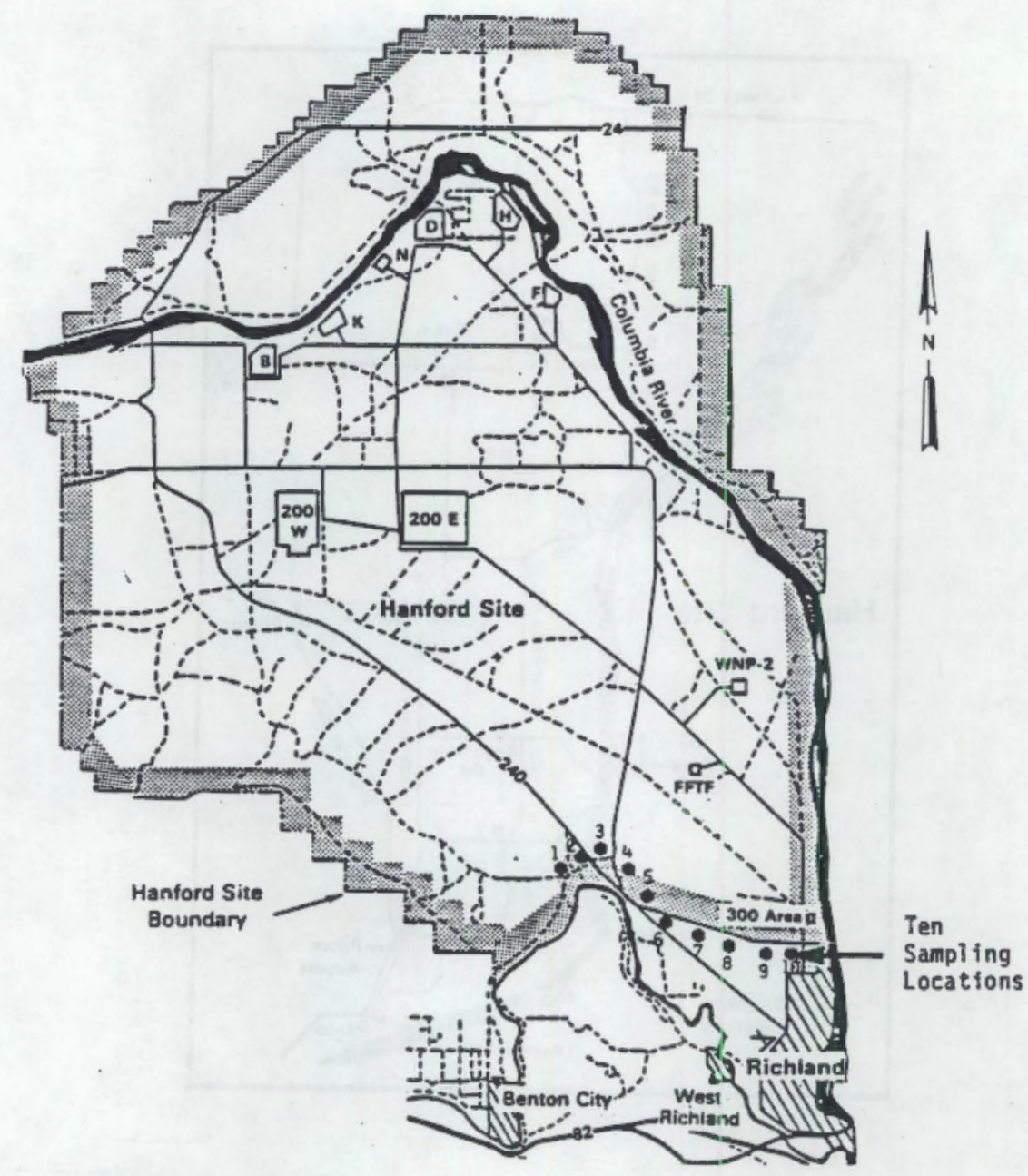

1985 Special Soil Samples - Southern Perimeter of the Hanford Site

\begin{tabular}{|c|c|c|c|c|c|}
\hline & & & Concentration, $\mathrm{pC} 1 / \mathrm{g}$ & & \\
\hline Sample & $\mathrm{Cs}-137$ & $5 r-90$ & $\mathrm{Pu}-238$ & $\mathrm{Pu}-239,240$ & Uranium \\
\hline$i$ & $0.03 \pm 0.02$ & $0.14 \pm 0.02$ & $0.0001 \pm 0.0001$ & $0.001 \pm 0.001$ & $0.42 \pm 0.14$ \\
\hline 2 & $0.60 \pm 0.04$ & $0.28 \pm 0.02$ & $0.0005 \pm 0.0004$ & $0.013 \pm 0.002$ & $0.22 \pm 0.07$ \\
\hline 3 & $0.03 \pm 0.02$ & $0.06 \pm 0.01$ & $0.0002 \pm 0.0003$ & $0.003 \pm 0.001$ & $0.32 \pm 0.12$ \\
\hline 4 & $1.6 \pm 0.1$ & $0.46 \pm 0.02$ & $0.0012 \pm 0.0004$ & $0.027 \pm 0.002$ & $0.24 \pm 0.09$ \\
\hline 5 & $0.43 \pm 0.04$ & $0.19 \pm 0.01$ & $0.0003 \pm 0.0003$ & $0.008 \pm 0.001$ & $0.23 \pm 0.09$ \\
\hline 6 & $0.14 \pm 0.03$ & $0.12 \pm 0.01$ & $0.0002 \pm 0.0002$ & $0.005 \pm 0.001$ & $0.18 \pm 0.07$ \\
\hline 7 & $0.37 \pm 0.03$ & $0.21 \pm 0.01$ & $0.0004 \pm 0.0003$ & $0.007 \pm 0.001$ & $0.16 \pm 0.07$ \\
\hline 8 & $0.07 \pm 0.02$ & $0.09 \pm 0.01$ & $0.0000^{(a)} \pm 0.0002$ & $0.004 \pm 0.009$ & $0.26 \pm 0.10$ \\
\hline 9 & $0.03 \pm 0.02$ & $0.07 \pm 0.01$ & $0.0000^{(a)} \pm 0.0003$. & $0.002 \pm 0.001$ & $0.26 \pm 0.09$ \\
\hline 10 & $0.85 \pm 0.05$ & $0.29 \pm 0.02$ & $0.0008 \pm 0.0002$ & $0.016 \pm 0.002$ & $0.24 \pm 0.09$ \\
\hline Prosser Barricade (b) & $0.73 \pm 0.05$ & $0.45 \pm 0.09$ & $0.0004 \pm 0.0003$ & $0.019 \pm 0.002$ & $0.80 \pm 0.24$ \\
\hline
\end{tabular}

(a) No net counts above background.

(b) 1985 results for closest routine sampling location.

FIGURE C.2. Unpublished Results from a Special-Purpose Study Along the Southern Perimeter of the Hanford Site, June 1985 


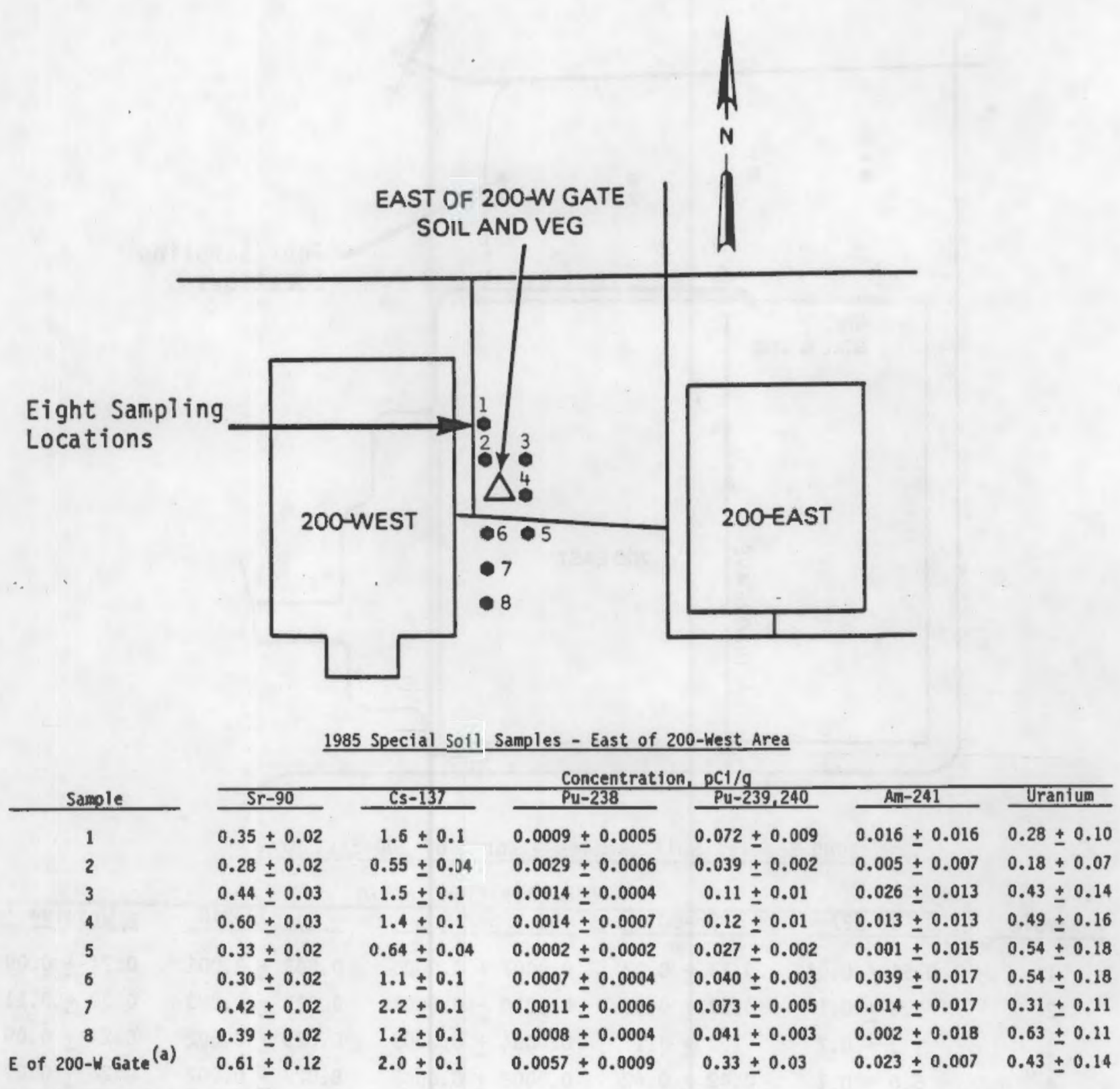

(a) 1985 results for closest routine sampiling location.

FIGURE C.3. Unpublished Results from a Special-Purpose Study East of the 200-West Area, August 1985 


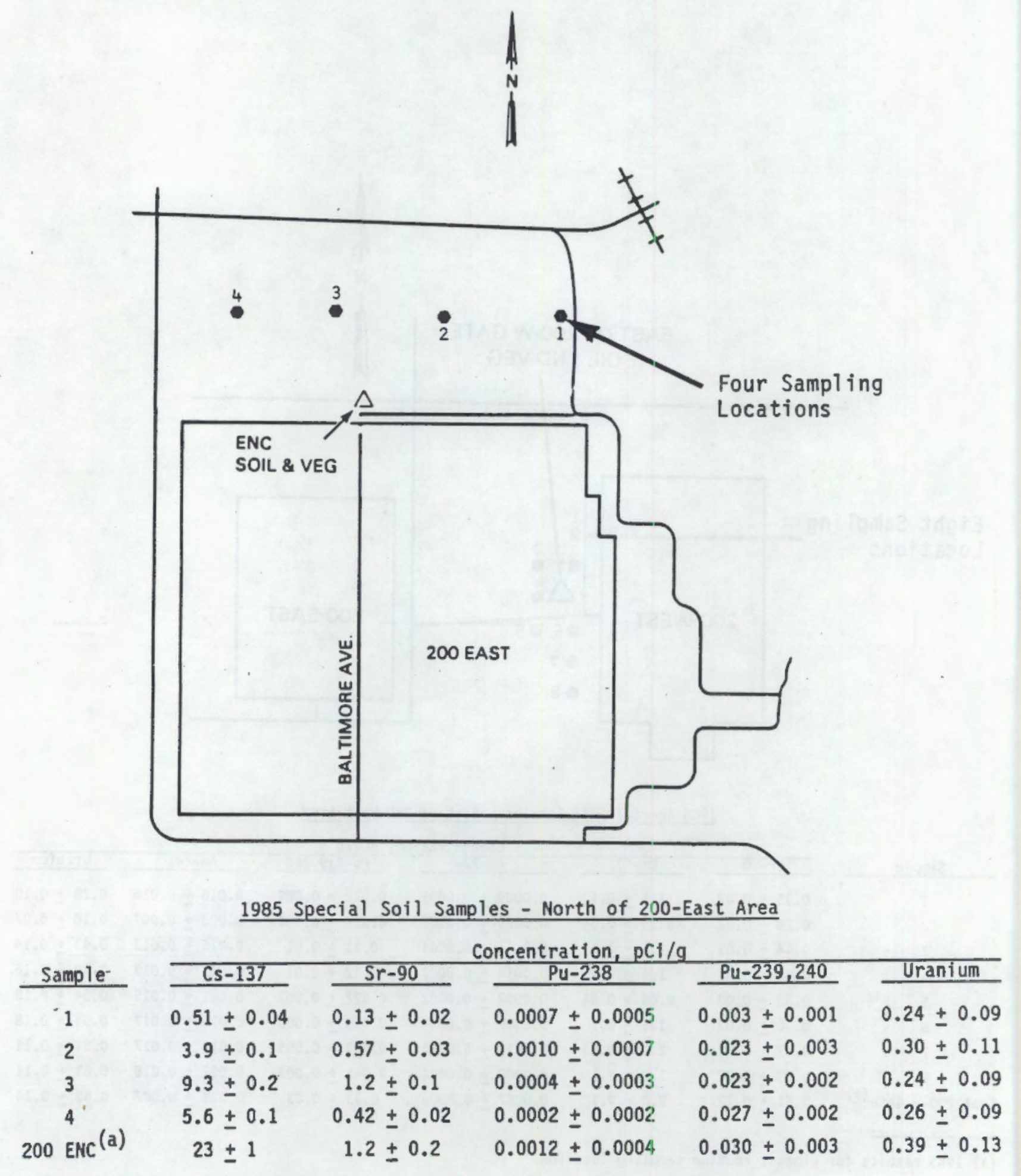

(a) 1985 results for closest routine sampling location.

FIEURE C.4. Unpublished Results from a Special-Purpose Study North of the 200-East Area, August 7985

$$
\text { C. } 4
$$




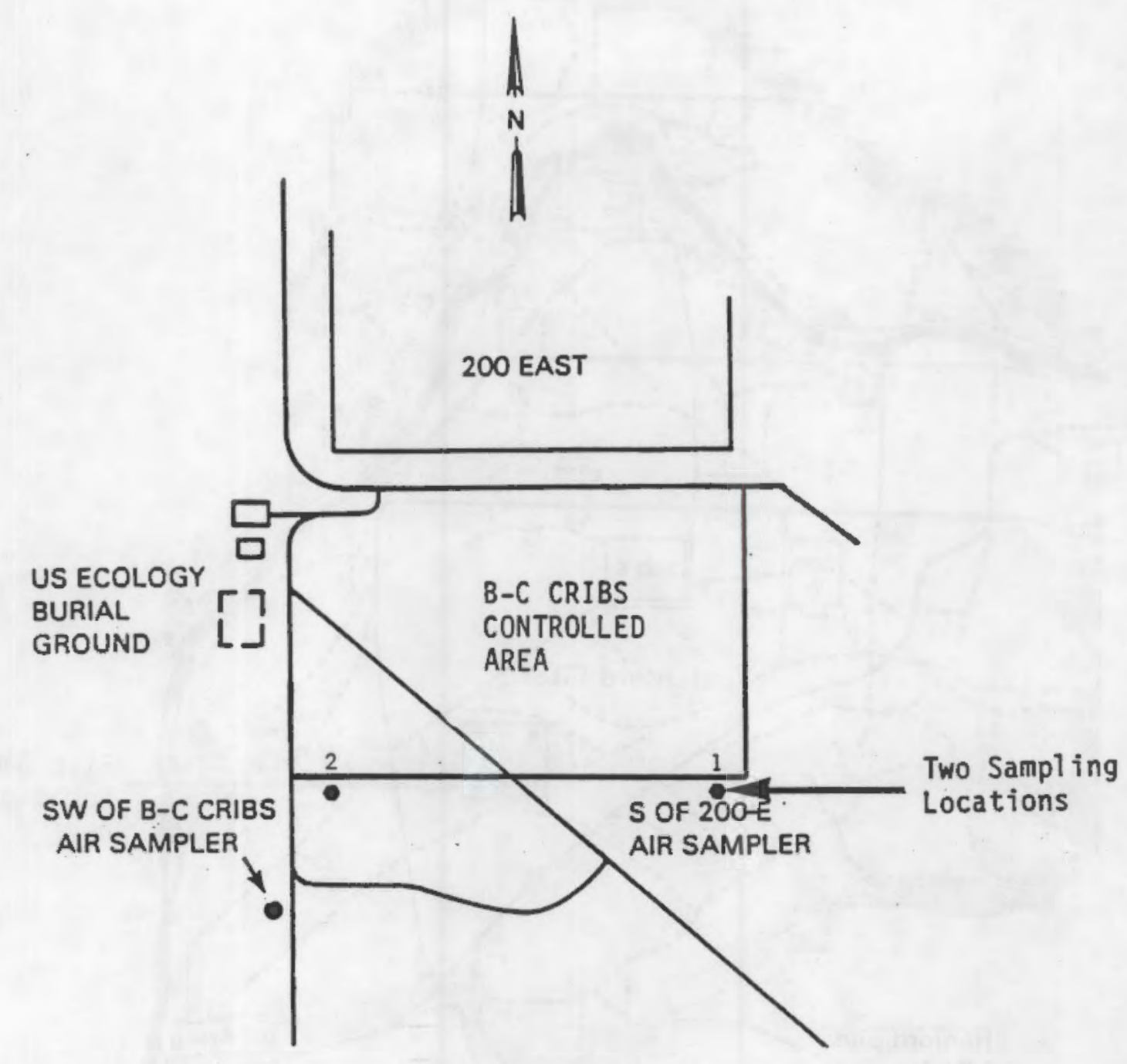

1985 Special Soil Samples - South of B-C Cribs Controlled Area

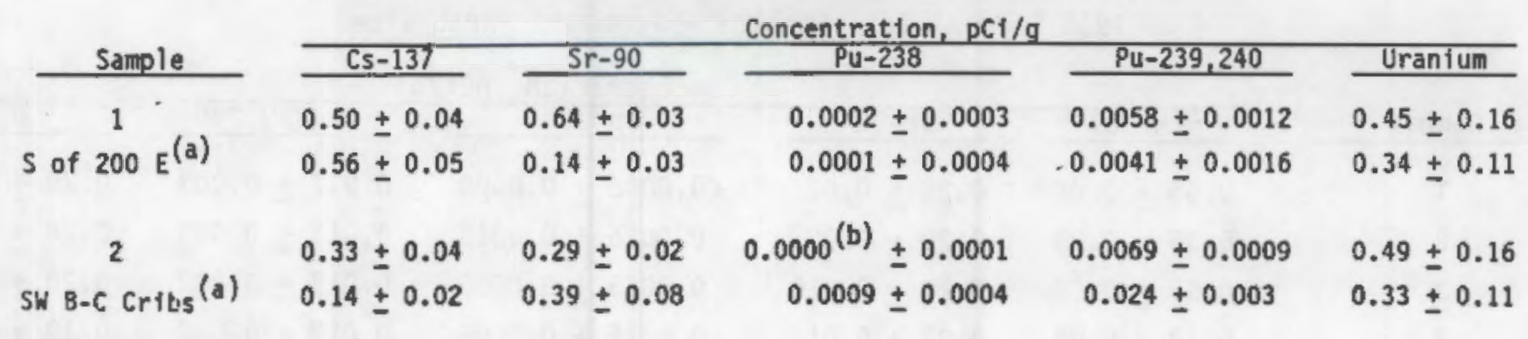

(a) 1985 results for closest routine sampling location.

(b) No net counts above background.

FIGURE C.5. Unpublished Results from a Special-Purpose Study South of the B-C Cribs Controlled Area, August 1985 


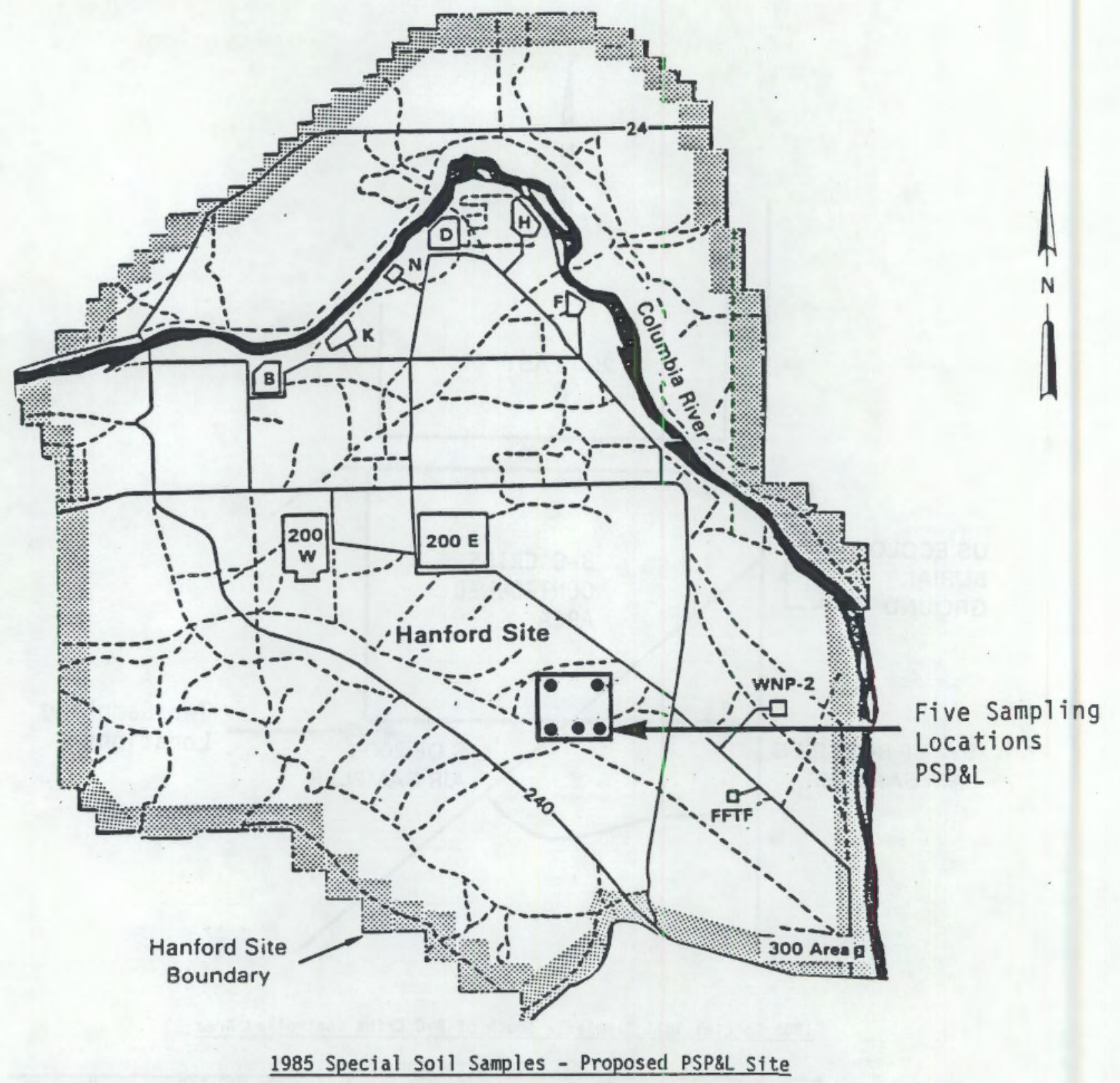

\begin{tabular}{|c|c|c|c|c|c|}
\hline & & & atration & & \\
\hline Sample & Cs-137 & $5 r-90$ & Pu-238 & $P_{y}-239,240$ & 0 \\
\hline 1 & $0.66 \pm 0.04$ & $0.26 \pm 0.02$ & $<0.0008 \pm 0.0009$ & $0.017 \pm 0.003$ & $0.29 \pm 0.10$ \\
\hline 2 & $0.36 \pm 0.03$ & $0.20 \pm 0.02$ & $0.0026 \pm 0.0015$ & $0.013 \pm 0.003$ & $0.28 \pm Q .10$ \\
\hline 3 & $0.55 \pm 0.04$ & $0.20 \pm 0.010$ & $0.0035 \pm 0.0010$ & $0.018 \pm 0.002$ & $0.20 \pm 0.07$ \\
\hline 4 & $0.70 \pm 0.04$ & $0.27 \pm 0.01$ & $0.0015 \pm 0.0009$ & $0.019 \pm 0.002$ & $0.19 \pm 0.07$ \\
\hline 5 & $0.31 \pm 0.03$ & $0.15 \pm 0.01$ & $0.0068 \pm 0.0008$ & $0.014 \pm 0.001$ & $0.28 \pm 0.10$ \\
\hline Barricade ${ }^{(a)}$ & $1.3 \pm 0.10$ & $0.31 \pm 0.06$ & $0.0006 \pm 0.0003$ & $0.017 \pm 0.002$ & $0.29 \pm 0.10$ \\
\hline
\end{tabular}

(a) 1985 results for closest routine sampling location.

FI SURE C.6. Unpubl ished Results from a Special-Purpose Study at the Proposed Puget Sound Power and Light Company Site, August 1985

C. 6 


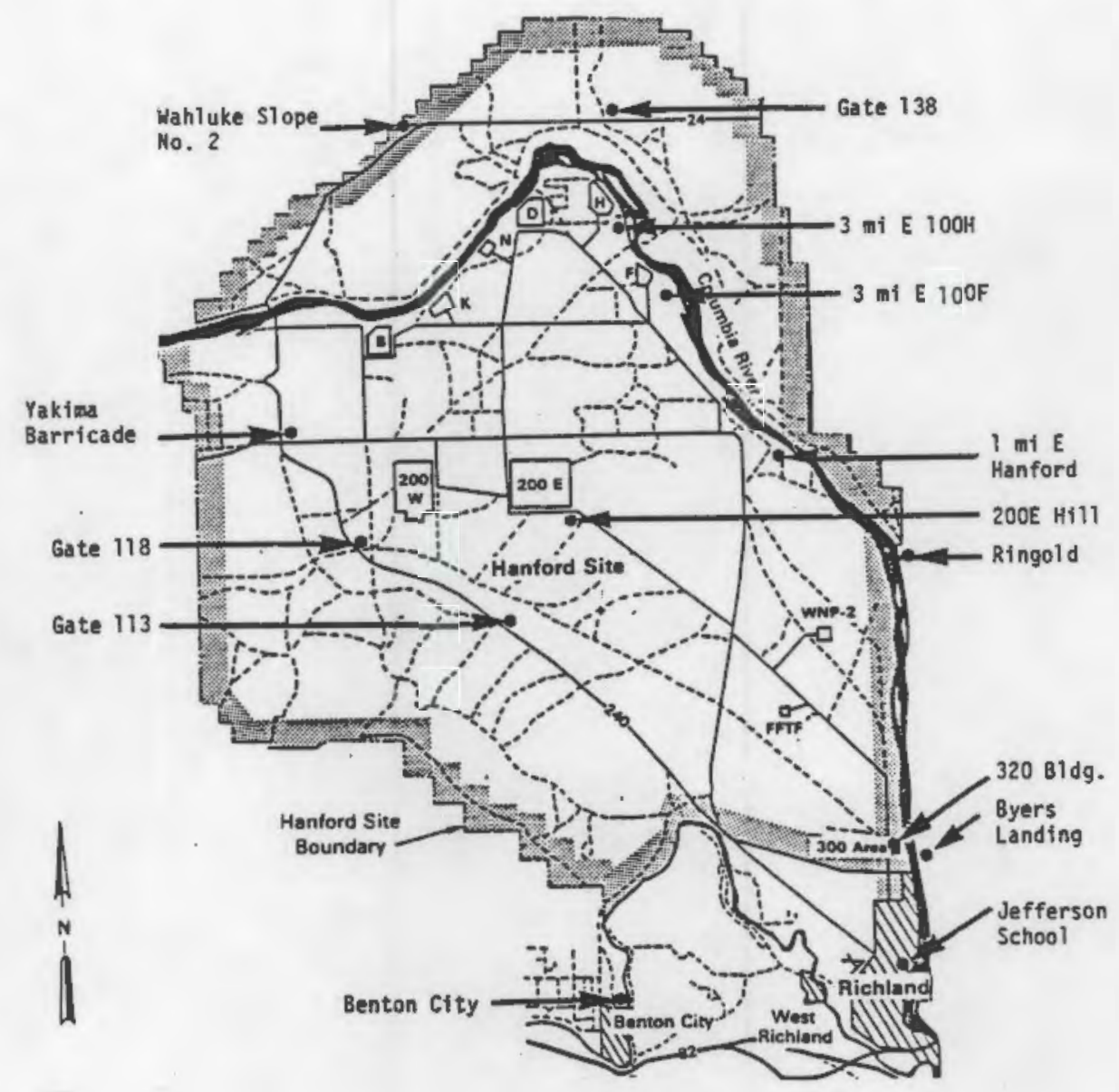

Eebruary and March 1970 Special Soil Samples

$(0.5$ inch deep) - Hanford Environs

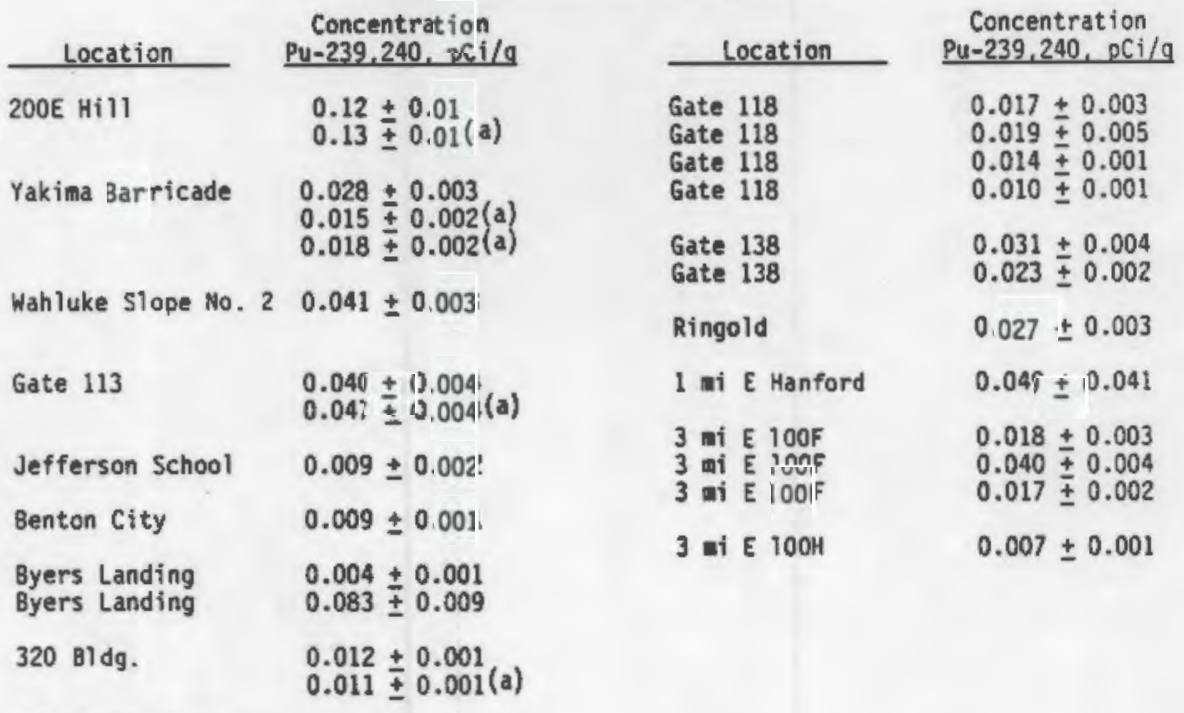

(a) Separate aliquot from single sample.

FIGURE C.7. Unpublished Results from a Special-Purpose Study at Various Onsite and Offsite Locations, February and March 1970 

PNL-6734

UC- $-11,41$

\section{DISTRIBUTION}

No. of

Copies

OFFSITE

10 DOE/Office of Scientific and Technical Information

3 R. R. Mooney

Washington State Department of Social and Health Services Radiation Protection

MS LE-13

Olympia, WA 98504-0095

\section{ONSITE}

8 DOE Richland Operations Office

R. F. Brich

D. L. Hoff

J. J. Sutey

M. W. Tiernan (5)

West inghouse Hanford Company

6 G. F. Booth

J. J. Dorian

R. E. Elder

E. M. Greager

R. E. Wheeler

WHC Files
No. of

Copies

46 Pacific Northwest Laboratory

T. L. Brown

L. L. Cadwell

D. H. Denham

R. L. Dirkes

J. W. Falco

R. H. Gray

H. A. Haerer

J. M. Hales

P. C. Hays

R. E. Jaquish

C. T. Kincaid

P. J. Mellinger

T. A. Nelson (2)

T. L. Page

K. R. Price (15)

W. R. Rickard

R. G. Riley

R. G. Schreckhise

R. J. Serne

R. L. Skaggs

J. A. Stott lemyre

R. E. Wildung

R. K. Woodruff

Historical File--R. K. Woodruff Publishing Coordination

Technical Report Files (5) 
$+$

= 